\title{
TESIS
}

\section{Estudio Analítico y Numérico de los Procesos de Fundición por Inyección a Presión}

\author{
por \\ Joaquín López Rodríguez \\ Ingeniero Industrial \\ presentada en la
}

ESCUELA TÉCNICA SUPERIOR DE INGENIERÍA INDUSTRIAL

para la obtención del

Grado de Doctor por la

UNIVERSIDAD POLITÉCNICA DE CARTAGENA

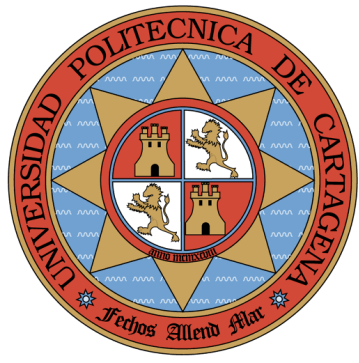

CARTAGENA, 9 de septiembre de 2002 



\title{
Estudio Analítico y Numérico de los Procesos de Fundición por Inyección a Presión
}

\author{
por: Joaquín López Rodríguez \\ Directores de la Tesis: Dr. Félix Faura Mateu \\ Dr. Julio Hernández Rodríguez \\ TRIBUNAL CALIFICADOR \\ Presidente: Dr. Antonio Crespo Martínez \\ Vocales: Dr. Jesús María Pérez García \\ Dr. Miguel Ángel Sebastián Pérez \\ Dr. Gerardo Trapaga \\ Dr. Javier García-Lomas Jung
}



Dedico este trabajo a la memoria de mi padre 



\section{Resumen}

Los procesos de fundición por inyección a presión presentan importantes limitaciones en lo que se refiere a la calidad de sus productos, debidas principalmente a la alta tendencia a la formación de porosidad en las piezas fabricadas. El mecanismo de formación de porosidad más importante en estos procesos es generalmente el atrapamiento de aire durante la etapa de llenado del molde. Las causas de atrapamiento de aire se pueden atribuir principalmente a las condiciones de operación que aumentan la ingestión de aire durante el tiempo de inyección y al uso de sistemas de evacuación de aire inadecuados. En la presente tesis doctoral se realiza un estudio sistemático de las condiciones de operación y características del sistema de inyección (fracción inicial de llenado, geometría de la cámara de inyección y ley de movimiento del pistón, fundamentalmente) y del sistema de evacuación (geometría y localización de los vientos, condiciones de evacuación de aire: atmosférica o al vacío, etc.) que minimizan los fenómenos de atrapamiento de aire y reducen en lo posible los tiempos de inyección, permitiendo esto último mejorar la productividad del proceso y evitar posibles solidificaciones prematuras del metal fundido durante la etapa de inyección. Además se determinan valores límite de las condiciones de operación que en todo caso deben evitarse para asegurar el correcto funcionamiento del proceso durante la etapa de inyección.

El flujo del metal fundido en el interior de la cámara de inyección se estudia mediante un modelo bidimensional en el que se desprecian los efectos viscosos y se introduce la aproximación de aguas poco profundas, que se resuelve numérica o analíticamente dependiendo de que se 
consideren o no los efectos de reflexión de la ola de metal fundido en la cámara de inyección. Este modelo se aplica a dos tipos de leyes de movimiento del pistón, correspondientes a aceleraciones exponencial y potencial en el tiempo, representativas de casos en que se programe la velocidad del pistón en función de la posición del pistón o del tiempo. En primer lugar se lleva a cabo un estudio analítico de los perfiles de la ola de metal fundido que se forma en la cámara de inyección, despreciando los posibles efectos de reflexión de dicha ola, para amplios rangos de los parámetros relevantes del proceso, para los que se determina el lugar y el instante en el que se forma una "discontinuidad" en la superficie libre del metal fundido (perfil de ola vertical). Los resultados de este estudio permiten determinar, para unas características geométricas del sistema de inyección y una fracción inicial de llenado de la cámara dadas, relaciones entre los parámetros que definen la ley de aceleración del pistón que reducen en lo posible el tiempo de inyección a la vez que evitan el atrapamiento de aire por rotura de la ola. Algunos resultados para la ley de aceleración exponencial obtenidos de esta forma se han comparado con los resultados teóricos y experimentales que han obtenido otros autores, observándose una buena concordancia entre ellos. Se observa que la ley de aceleración exponencial produce perfiles de ola más inclinados en el momento de formación de la discontinuidad y por lo tanto requiere tiempos de llenado más pequeños que la ley de aceleración potencial. En cualquier caso, se comprueba que ninguna de estas leyes permite elegir un conjunto de parámetros que elimine totalmente el aire del interior de la cámara de inyección antes de que la ola se rompa o se refleje contra la pared final de la cámara de inyección. Para tener en cuenta los efectos de reflexión, el modelo de aguas poco profundas se resuelve numéricamente, lo que permite determinar la influencia de los parámetros que caracterizan la ley de aceleración exponencial sobre el área ocupada por el aire en la cámara de inyección en el momento en el que el metal fundido alcanza el conducto de entrada hacia la cavidad del molde. La concordancia entre los parámetros que satisfacen el doble compromiso de reducción de aire y de los tiempos de inyección obtenidos con este modelo y los correspondientes al modelo que no tiene en cuenta la reflexión de la ola confirma la validez del modelo más sencillo para conseguir predicciones cualitativamente correctas de parámetros óptimos del proceso. A partir del modelo analítico, se determina además una nueva ley de aceleración que permitiría obtener un perfil de ola completamente vertical en el instante en el que ésta alcanza la pared final de la cámara de inyección, lo que reduciría a cero la cantidad de aire atrapado y minimizaría el tiempo de llenado. Se comprueba que esta ley presenta restricciones algo mayores respecto al máximo valor de la fracción inicial de llenado que se podría usar para evitar que el metal fundido pueda ser expulsado a través del orificio de colada, y por otro lado permite, a diferencia de las otras leyes estudiadas 
en esta tesis, alcanzar la velocidad crítica del pistón antes de que se inicie la rotura de la ola para cualquier valor de la fracción inicial de llenado de la cámara de inyección.

La importancia de los efectos viscosos y no hidrostáticos en la cámara de inyección se evalúa utilizando un código de elementos finitos basado en el método VOF para determinar la posición de la superficie libre en cada instante, que utiliza una malla de cálculo que se mueve y deforma usando una técnica similar a la utilizada en el método LagrangianoEuleriano arbitrario. Se observa que estos efectos pueden llegar a ser importantes una vez que el metal fundido alcanza la pared final o el techo de la cámara de inyección, donde se puede formar un chorro de pared para determinadas condiciones de proceso. En general, los resultados numéricos que se obtienen considerando estos efectos predicen perfiles de ola más suaves y un ligero retardo de la rotura de la ola, aunque, a pesar de estas diferencias, las predicciones del volumen de aire atrapado obtenidas con el modelo de aguas poco profundas describen razonablemente bien la influencia de los parámetros de aceleración y de la fracción inicial de llenado sobre dicho volumen.

El flujo en los sistemas de evacuación se analiza, para condiciones de evacuación atmosférica y al vacío, utilizando un modelo no estacionario en el que el aire se considera un gas perfecto y el flujo a través de los vientos se supone unidimensional y con fricción. Para situaciones en las que los efectos no estacionarios no son importantes, se comprueba que existe una buena concordancia entre los resultados de este modelo y los obtenidos con modelos quasi-estacionarios previos, excepto para condiciones de evacuación al vacío, en las que los modelos quasi-estacionarios sólo son válidos para presiones de vacío suficientemente bajas. Se demuestra que existen rangos de condiciones habituales de operación, particularmente en sistemas de evacuación al vacío, en las que los efectos no estacionarios se deben tener en cuenta para elegir los parámetros de diseño que minimicen la cantidad de aire atrapado y de este modo reduzcan la porosidad en las piezas fabricadas. Se comprueba además que el valor del área del viento que proporciona una buena evacuación de aire, y por encima del cual no se consigue una disminución adicional apreciable de la masa de aire residual en la cavidad del molde, es generalmente mayor que el obtenido a partir del modelo quasi-estacionario, y que la diferencia entre ambos aumenta al aumentar la no estacionariedad del proceso, consiguiéndose una reducción adicional de la masa de aire atrapado por encima de la estimada a partir del modelo quasi-estacionario. 



\section{Agradecimientos}

Mi más profundo agradecimiento lo dirijo a mis directores de tesis, Dr. Félix Faura Mateu y Dr. Julio Hernández Rodríguez, por la inestimable ayuda prestada y el constante estímulo que de ellos he recibido.

Quiero agradecer también la colaboración de todas las personas que forman parte del Departamento de Ingeniería de Materiales y Fabricación de la Universidad Politécnica de Cartagena, y en particular, agradezco a Rosendo Zamora Pedreño los prácticos consejos informáticos que me ha prestado. Asimismo agradezco al Departamento de Mecánica de la UNED, y en particular a Pablo Gómez del Pino, la colaboración dispensada durante mis estancias en dicho Departamento.

A Gerald P. Backer, creador del código de elementos finitos Wrafts, le agradezco su ayuda en la utilización de dicho código.

También deseo expresar mi agradecimiento a la Comisión Interministerial de Ciencia y Tecnología (CICYT) por la financiación concedida al proyecto de investigación titulado "Estudio técnico-económico y optimización de procesos de fundición por inyección a alta presión mediante técnicas computacionales" (TAP97-0489), en cuyo marco se ha realizado esta tesis.

A las empresas Robert Bosch España, Empresa Nacional Bazán de Construcciones Navales Militares, Ruffini, Suzuki-Santana, Fagor Ederlan y a la Asociación Técnica y Desarrollo de la Fundición a Presión Española (TEDFUN), les agradezco su colaboración y asesoramiento durante el desarrollo del proyecto de investigación anteriormente mencionado.

Finalmente agradezco a mi familia, mi madre y mi hermana, su apoyo y aliento. 



\section{Índice general}

Resumen vii

Agradecimientos $\quad$ xi

Índice de tablas $\quad$ xvii

Índice de figuras $\quad$ xxvii

Nomenclatura $\quad$ xxix

1 Introducción 1

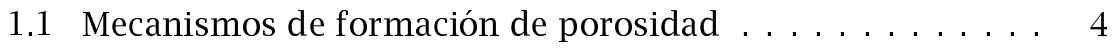

1.2 Tecnología de los procesos FIAP $\ldots \ldots \ldots \ldots \ldots$

1.2.1 Sistema de inyección $\ldots \ldots \ldots \ldots \ldots$

1.2.2 Sistema de evacuación de aire . . . . . . . . . 15

1.2.2.1 Área de la sección transversal de los vientos 18

1.3 Aplicación de modelos CFD a procesos de fundición . . . . 20

1.4 Objetivos de la tesis . . . . . . . . . . . . . 24

2 Estudio analítico del movimiento del metal fundido en la cámara 27

2.1 Modelo analítico . . . . . . . . . . . . . . . . . 28

2.2 Movimiento del pistón con aceleración exponencial . . . . . 32

2.2.1 Instante y lugar de formación de la discontinuidad . . 33

2.2.2 Fracción inicial de llenado mínima . . . . . . . . . . 34

2.2.3 Parámetros óptimos de aceleración . . . . . . . . . 36 
2.2.4 Comparación con otros resultados analíticos y experimentales .................. . 43

2.3 Movimiento del pistón con aceleración potencial . . . . . . . 46

2.4 Ley óptima de movimiento del pistón . . . . . . . . . . . . 53

2.5 Límite máximo de la fracción inicial de llenado . . . . . . . . . 58

2.6 Conclusiones . . . . . . . . . . . . . . . 66

3 Efectos de reflexión de la ola de metal fundido en la cámara $\quad 69$

3.1 Modelo analítico con efectos de reflexión . . . . . . . . 69

3.2 Resolución numérica del modelo . . . . . . . . . . . 76

3.2.1 Procedimiento numérico . . . . . . . . . . . 76

3.2.1.1 Interior de la cámara de inyección . . . . . . 81

3.2.1.2 Superficie del pistón . . . . . . . . . . . . 83

3.2.1.3 Pared final de la cámara de inyección . . . . . 84

3.2.2 Precisión del modelo numérico . . . . . . . . . . . . 85

3.3 Discusión de resultados . . . . . . . . . . . . . . 88

3.3.1 Perfiles de la superficie libre de metal fundido . . . . 88

3.3.2 Volumen de aire atrapado . . . . . . . . . . . . . 92

3.3.2.1 Elección de las condiciones de operación . . . 95

3.4 Conclusiones . . . . . . . . . . . . . . . . 98

4 Estudio numérico del movimiento del metal fundido en la cámara 99

4.1 Modelo numérico . . . . . . . . . . . . . . . . . 100

4.1.1 Ecuaciones de conservación y condiciones de contorno100

4.1 .2 Procedimiento numérico . . . . . . . . . . . . . . . 101

4.1.3 Estudio de la independencia de la malla . . . . . . . . 101

4.2 Discusión de resultados . . . . . . . . . . . . . . . . . 105

4.2.1 Comparación con los modelos de aguas poco profundas105

4.2.1.1 Perfiles de la superficie libre del metal fundido105

4.2.1.2 Cierre de la entrada hacia la cavidad del molde108

4.2.1.3 Volumen de aire atrapado . . . . . . . . . 110

4.2.1.4 Límites de la fracción inicial de llenado . . . . 115

4.2.2 Comparación con resultados experimentales . . . . . . 115

4.3 Conclusiones . . . . . . . . . . . . . . . . . . . . . 121

5 Estudio de la evacuación de aire a través de los vientos 123

5.1 Modelo quasi-estacionario . . . . . . . . . . . . . . . . . 124

5.2 Modelo no estacionario . . . . . . . . . . . . . . . . 126

5.3 Resolución numérica del modelo no estacionario . . . . . . 129

5.3.1 Discretización de las ecuaciones características y de compatibilidad . . . . . . . . . . . . . . 131

5.3.2 Descripción del procedimiento numérico . . . . . . . 132

5.3.2.1 Interior del viento . . . . . . . . . . . . . 136

5.3.2.2 Sección de entrada al viento . . . . . . . . . 138

5.3.2.3 Sección de salida del viento . . . . . . . . . 140 
5.3.3 Procedimiento de interpolación de las propiedades en los puntos iniciales . . . . . . . . . . . . . 142

5.3.4 Estudio de la precisión del procedimiento numérico . 145

5.3 .5 Otros aspectos numéricos . . . . . . . . . . . 152

5.4 Discusión de resultados de los modelos de evacuación de aire155

5.4 .1 Evacuación atmosférica . . . . . . . . . . . . 155

5.4 .2 Evacuación al vacío . . . . . . . . . . . . . . . . 164

5.5 Conclusiones . . . . . . . . . . . . . . . . . . . 172

6 Conclusiones finales 175

6.1 Conclusiones . . . . . . . . . . . . . . . . . . . . . . 175

6.2 Desarrollos futuros . . . . . . . . . . . . . . . . . . 180

Apéndice A. Ecuaciones características y condiciones de compati-

bilidad

A.1 Flujo en aguas poco profundas . . . . . . . . . . . . 183

A.2 Flujo unidimensional no estacionario de un gas perfecto $\ldots 187$

Bibliografía

191

Biografía del autor 



\section{Índice de tablas}

1.1 Características de diversos procesos de fundición. . . . . . 3

1.2 Lista parcial de códigos de fundición y de propósito general usados para simulación de procesos de fundición. . . . . . 23

2.1 Expresiones analíticas de la velocidad crítica. . . . . . . . . 36 



\section{Índice de figuras}

1.1 Esquema de una máquina de fundición por inyección a alta presión con cámara caliente (basado en Kalpakjian (1995)). . 8

1.2 Esquema de una máquina de fundición por inyección a alta presión con cámara fría horizontal (basado en Kalpakjian (1995)). . . . . . . . . . . . . . . . . . . . .

1.3 Fases de la etapa de inyección en los procesos FIAP con cámaras horizontales. a) Fase lenta. b) Fase rápida. c) Intensificación de la presión. . . . . . . . . . . . . . . . . 1

1.4 Condiciones que tienden a incrementar el atrapamiento de aire: a) velocidad del pistón superior a la velocidad crítica; b) velocidad del pistón inferior a la velocidad crítica. . . . . 12

1.5 Diagrama típico de la presión del sistema de inyección y el desplazamiento del pistón durante el llenado del molde (basado en Street (1977)). . . . . . . . . . . . . . . . . . 14

1.6 Detalle esquemático de un rebosadero y un viento. . . . . . 16

1.7 Esquema de un sistema de vacío en máquinas de fundición por inyección a presión. . . . . . . . . . . . . . 17

1.8 Modelado tridimensional de la tapa del cárter de un motor Diesel fabricado en Suzuki-Santana Motor, S.A. . . . . . . . . 21

1.9 Resultados del llenado del molde correspondiente al modelo tridimensional de la Figura 1.8, obtenidos con el código de elementos finitos Wrafts para distintas velocidades de inyección. . . . . . . . . . . . . . . . 22

2.1 Esquema de una máquina de fundición por inyección a alta presión con cámara de inyección horizontal. . . . . . . . . 29 
2.2 Esquema del problema y sistema de coordenadas. . . . . . . 29

2.3 Diagrama de las líneas características del problema. . . . . . 30

2.4 Fracción inicial de llenado mínima, $f_{\min }$, en función de $\xi$, para la ley de movimiento de la Ecuación (2.15). . . . . . . 35

2.5 Perfiles de la ola en $t=t_{c}$, satisfaciendo la condición $x_{c}=L$, para la ley de movimiento de la Ecuación (2.15) y diferentes valores de la fracción inicial de llenado y $\xi$. . . . . . . . . . 39

2.6 Tiempo adimensional de llenado en función de $\xi$ para diferentes valores de $f$ con la ley de movimiento de la Ecuación (2.15). . . . . . . . . . . . . . . . . .

2.7 Área adimensional ocupada por el aire en la cámara de inyección en $t=t_{c}$, en función de $\xi$, para una ley de aceleración exponencial con la que se obtiene $x_{c}=L \ldots \ldots \ldots$. . . 42

2.8 Comparación de los valores óptimos de $\alpha$ predichos en este trabajo con el modelo analítico sin reflexión y los obtenidos por Tszeng y Chu (1994) para una carrera del pistón $L=45,72 \mathrm{~cm}$ (18 in), con las mediciones experimentales de Duran et al. (1991). . . . . . . . . . . . . . . . . . . . 43

2.9 Medidas experimentales del volumen de aire atrapado de Duran et al. (1991). . . . . . . . . . . . . . . . . . . . . . . . 45

2.10 Fracción inicial de llenado mínima, $f_{\min }$, en función de $n$, para la ley de movimiento de la Ecuación (2.42). . . . . . . . 48

2.11 Tiempo de llenado adimensional en función de $n$ para diferentes valores de $f$ y la ley de movimiento de la Ecuación (2.42), para condiciones de operación que satisfacen $x_{c}=L$.

2.12 Distancia recorrida por la parte superior del frente de ola en $t_{c}$, adimensionalizada con $L$, en función de la fracción inicial de llenado para $x_{c}=L$ y las leyes de movimiento de las Ecuaciones (2.15) y (2.42), con $\xi=\frac{4}{3}$ y $n=1$, respectivamente. . . . . . . . . . . . . . . . .

2.13 Área adimensional ocupada por el aire en la cámara de inyección en $t=t_{c}$, en función de $n$, para una ley de aceleración potencial en la que $x_{c}=L \ldots \ldots \ldots \ldots$. . . . . 52

2.14 Perfiles de la ola para los parámetros de las leyes de aceleración de las Ecuaciones (2.16) y (2.43) que satisfacen la condición $x_{c}=L$ y minimizan el tiempo de llenado $t_{f}$. a)

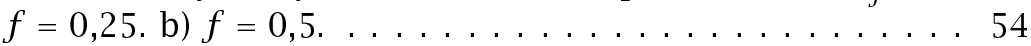

2.15 Esquema de la evolución de las características para que el perfil de la ola sea completamente vertical en $x=x_{c}=L . ~ . ~ .55$

2.16 Evolución del perfil de la ola con $t / t_{c}$ para diferentes valores de la fracción inicial de llenado y la ley de aceleración óptima del pistón de la Ecuación (2.61) para $\ell=L . \ldots \ldots \ldots$ 
2.17 Comparación entre los perfiles de la superficie de la ola obtenidos analíticamente y los obtenidos numéricamente para la ley de aceleración de la Ecuación (2.61) con $\ell=L$ y $X^{\prime}=u_{\eta}$ para $t>t_{\eta} . \eta=5,08 \mathrm{~cm}, H / \eta=1,3, f=0,254 \mathrm{y} \ell / \eta=9$. .59

2.18 Esquema de la cámara de inyección. . . . . . . . . . 60

2.19 Fracción inicial de llenado máxima en función de $X_{p} / L$, para la ley aceleración exponencial y diferentes valores de $\xi$. Comparación con la ley de aceleración de la Ecuación (2.61). 61

2.20 Fracción inicial de llenado máxima en función de $X_{p} / L$, para la ley de aceleración potencial y diferentes valores de $n$. Comparación con la ley de aceleración de la Ecuación (2.61). 63

2.21 Fracción inicial de llenado máxima en función de $\xi$, para diferentes valores de $X_{p} / L$, para la ley de aceleración exponencial. . . . . . . . . . . . . . . . . . . 64

2.22 Fracción inicial de llenado máxima en función de $n$, para diferentes valores de $X_{p} / L$, para la ley de aceleración potencial. . . . . . . . . . . . . . . . . . 65

3.1 Esquema de la evolución de las características considerando los efectos de la reflexión del metal en la pared final de la cámara $(x=L) . \ldots \ldots \ldots \ldots \ldots \ldots \ldots \ldots \ldots$

3.2 Curva $B C$ correspondiente a las Ecuaciones (3.10) y (3.10) para diferentes fracciones iniciales de llenado, $f$, y valores del parámetro $\xi$ satisfaciendo la condición $x_{c}=L$ según el modelo analítico sin reflexión. . . . . . . . . . . . . 74

3.3 Diagrama de flujo de la subrutina PISTÓN para calcular las propiedades del flujo en un punto situado en la superficie del pistón. . . . . . . . . . . . . . . . . 78

3.4 Diagrama de flujo de la subrutina INTER para calcular las propiedades del flujo en un punto situado en el interior de la cámara de inyección. . . . . . . . . . . . . . . . 79

3.5 Diagrama de flujo de la subrutina PARED para calcular las propiedades del flujo en un punto situado en la pared final de la cámara de inyección. . . . . . . . . . . . . 80

3.6 Malla de diferencias finitas en el plano $x t$ para un punto situado en el interior de la cámara de inyección. . . . . . . 81

3.7 Malla de diferencias finitas en el plano $x t$ para un punto situado en la superficie del pistón. . . . . . . . . . . 83

3.8 Malla de diferencias finitas en el plano $x t$ para un punto situado en la pared final de la cámara de inyección. . . . . . . 84 
3.9 Error numérico de la Ecuación (3.39) en función del tamaño adimensional de la malla $\Delta x / L$, en la sección intermedia entre la superficie del pistón y la pared final de la cámara, y $t=L / \mathcal{C}_{0}(f=0,5$ y ley de movimiento del pistón de la Ecuación (2.42) con $\gamma=32,7) \ldots \ldots \ldots$. . . . . 86

3.10 Error relativo al refinamiento de la malla definido por la Ecuación (3.40) en función del tamaño adimensional de la malla $\Delta x / L$, en diferentes secciones de la cámara y $t c_{0} / L=$ 1,08 (ley de aceleración del pistón de la Ecuación (2.16), $f=0,25, \xi=3$ у $L / \beta=9,46 . \ldots \ldots \ldots \ldots$

3.11 Perfiles de la ola en el instante en el que se forma una discontinuidad para la ley de aceleración del pistón de la Ecuación (2.16), con diferentes valores de $\xi$ y distintas fracciones iniciales de llenado, que satisfacen la condición $x_{c}=0,975 \mathrm{~L}$, según el modelo analítico sin reflexión. . . . . . . . . . . 89

3.12 Comparación entre los resultados de los perfiles para condiciones en las que la ola reflejada empieza a romperse en el instante en el que el metal fundido alcanza la entrada hacia la cavidad del molde y los obtenidos despreciando los efectos de reflexión. a) $f=0,25$, b) $f=0,3 . \ldots \ldots$. . . 9 90

3.13 Comparación entre los resultados de los perfiles para condiciones en las que la ola reflejada empieza a romperse en el instante en el que el metal fundido alcanza la entrada hacia la cavidad del molde y los obtenidos despreciando los efectos de reflexión. a) $f=0,4$, b) $f=0,5 \ldots \ldots \ldots$. . . .

3.14 Resultados del área adimensional ocupada por el aire cuando el metal fundido alcanza la entrada hacia la cavidad del molde y del tiempo de llenado adimensional en función de $L / \beta$, para diferentes valores de $\xi$, y $f=0,25 \ldots \ldots \ldots 9$

3.15 Resultados del área adimensional ocupada por el aire cuando el metal fundido alcanza la entrada hacia la cavidad del molde y del tiempo de llenado adimensional en función de $L / \beta$, para diferentes valores de $\xi$, y $f=0,4 \ldots \ldots 94$

3.16 Resultados del valor del área adimensional $A /(H L)$ y del tiempo de llenado en función de $\xi$, para diferentes fraccio-

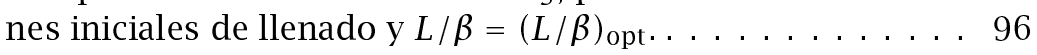

3.17 Relación entre el valor óptimo $(L / \beta)_{\text {opt }}$ y los valores de las Ecuaciones (2.33) y (2.35) correspondientes al modelo analítico sin reflexión, en función de $\xi$ para diferentes fracciones iniciales de llenado. . . . . . . . . . . . . . . . 97

4.1 Malla de cálculo en la cámara de inyección. . . . . . . . . . 102 
4.2 Influencia del tamaño de la malla en los perfiles de la superficie libre del metal fundido. Ley de aceleración de la Ecuación (2.61) para $t \leq t_{\eta}$ y $X^{\prime}=u_{\eta}$ para $t>t_{\eta}$, con $\eta=5,08 \mathrm{~cm}, f=0,254, \ell / \eta=9, L / \ell=1,56$ y $H / \eta=1,3 . \ldots 103$

4.3 Comparación entre los resultados analíticos de los perfiles de la superficie libre de metal fundido en distintos instantes, y los resultados numéricos obtenidos utilizando diferentes tamaños de malla. Ley de aceleración de la Ecuación (2.61) con $\ell=L$ y $X^{\prime}=u_{\eta}$ para $t>t_{\eta}$, con $\eta=5,08 \mathrm{~cm}, \ell / \eta=9$,

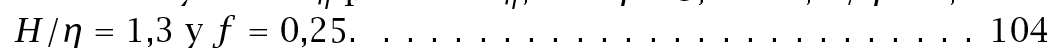

4.4 Comparación entre resultados numéricos y analíticos para los perfiles de la superficie libre. Ley de aceleración del pistón de la Ecuación (2.61) para $t \leq t_{\eta}$ y $X^{\prime}=u_{\eta}$ para $t>t_{\eta}$. $\eta=5,08 \mathrm{~cm}, \ell / \eta=9, L / \ell=1,56, H / \eta=1$. a) $f=0,254$. b)

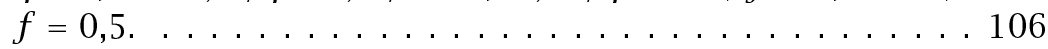

4.5 Resultados numéricos de los perfiles de la ola para la ley de aceleración de la Ecuación (2.61) $\left(t \leq t_{\eta}\right)$ y $X^{\prime}=u_{\eta}$ para $t>t_{\eta}$, con $t \geq t_{c}, \eta=5,08 \mathrm{~cm}, \ell / \eta=9, L / \ell=1,56, H / \eta=1$.

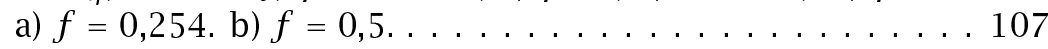

4.6 Comparación entre los resultados de los perfiles de ola obtenidos con el modelo de aguas poco profundas, para condiciones que hacen que la ola reflejada empiece a romperse en el instante en el que el metal fundido alcanza la entrada hacia la cavidad del molde, y los correspondientes resultados numéricos obtenidos con el código Wrafts. a) $f=0,25$,

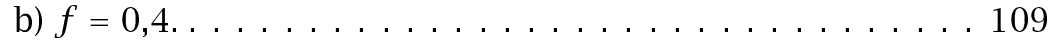

4.7 Resultados del área adimensional ocupada por el aire cuando el metal fundido alcanza la entrada hacia la cavidad del molde, obtenidos con el código Wrafts y con el modelo de aguas poco profundas, y del tiempo adimensional de llenado en función de $L / \beta$, para diferentes valores de $\xi, L / H=9$

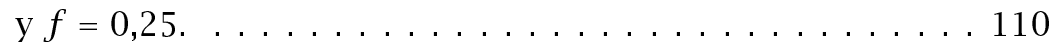

4.8 Resultados del área adimensional ocupada por el aire cuando el metal fundido alcanza la entrada hacia la cavidad del molde, obtenidos con el código Wrafts y con el modelo de aguas poco profundas, y del tiempo adimensional de llenado en función de $L / \beta$, para diferentes valores de $\xi, L / H=9$

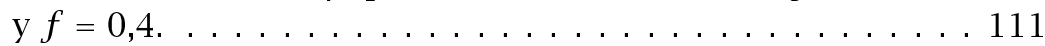

4.9 Valores del área mínima adimensional $A /(H L)$ ocupada por el aire cuando el metal fundido alcanza la entrada hacia la cavidad del molde, obtenidos con el código Wrafts, y del tiempo adimensional de llenado en función de $\xi \operatorname{con} L / H=$ 9. a) $f=0,25$. b) $f=0,4 \ldots \ldots \ldots \ldots \ldots \ldots \ldots \ldots$ 
4.10 Resultados numéricos de la evolución de la superficie libre del metal fundido para la ley de aceleración de la Ecuación (2.61) con $\ell=L$ y $L / H=6$. a) $f=0,4$. b) $f=0,25$. . . . . . 114

4.11 Comparación entre los resultados analíticos (línea continua) y numéricos (contorno gris) de los perfiles de la superficie libre en distintos instantes. Ley de aceleración de la Ecuación (2.61) con $\ell=L$ para $t \leq t_{H}, X^{\prime}=u_{H}$ para $t>t_{H}$, $f=0,5, L / H=9$ y $X_{p} / L=0,4 \ldots \ldots \ldots \ldots \ldots \ldots$

4.12 Comparación entre los resultados analíticos y numéricos de la altura del metal fundido en la superficie del pistón en función del tiempo $\left(t \leq t_{H}\right)$, para diferentes fracciones iniciales de llenado, con la ley de aceleración de la Ecuación (2.61) para $\ell=L$, y $L / H=9 . \ldots \ldots \ldots \ldots \ldots \ldots \ldots \ldots$

4.13 Resultados numéricos y analíticos de los perfiles de la superficie libre del metal fundido para la ley de aceleración de la Ecuación (2.16). $L / \beta=18, L / H=9, H=5,08 \mathrm{~cm}$, $f=0,254$ y diferentes valores de $\alpha \ldots \ldots \ldots \ldots . \ldots 118$

4.14 Resultados numéricos y analíticos de los perfiles de la superficie libre del metal fundido para la ley de aceleración de la Ecuación (2.16). $L / \beta=18, L / H=9, H=5,08 \mathrm{~cm}, f=0,5$

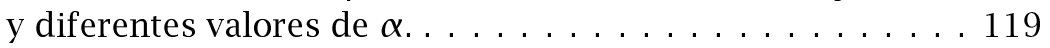

4.15 Comparación entre resultados analíticos, numéricos y experimentales para el parámetro de aceleración óptimo del pistón, $\alpha$, en función de la fracción inicial de llenado con $L / \beta=18, L / H=9$ y $H=5,08 \mathrm{~cm} . \ldots \ldots \ldots \ldots$

5.1 Modelo simplificado de evacuación de aire a través de los vientos (Bar-Meir et al., 1996, 1997). . . . . . . . . . . . . . 124

5.2 Esquema simplificado del sistema de evacuación de aire. . . 126

5.3 Esquema del viento y de la malla de diferencias finitas en el plano $x t . \ldots \ldots \ldots \ldots \ldots \ldots$

5.4 Diagrama de flujo para el cálculo de las propiedades del aire en la sección de entrada al viento. . . . . . . . . . . . . . 133

5.5 Diagrama de flujo para el cálculo de las propiedades del aire en el interior del viento. . . . . . . . . . . . . . . . . . . . 134

5.6 Diagrama de flujo para el cálculo de las propiedades del aire en la sección de salida del viento. . . . . . . . . . . . 135

5.7 Malla de diferencias finitas en el plano $x t$ para un punto situado en el interior del viento. . . . . . . . . . . . 137

5.8 Malla de diferencias finitas en la sección de entrada al viento para condiciones de flujo subsónico. . . . . . . . . . . 141

5.9 Malla de diferencias finitas en la sección de salida del viento.

a) Flujo subsónico. b) Flujo supersónico. . . . . . . . . . . . 143

5.10 Malla de diferencias finitas. . . . . . . . . . . . . . 144 
5.11 Resultados de una interpolación lineal y una interpolación natural cúbica spline para la velocidad adimensional del aire a lo largo del viento en $t=0,452 t_{f}$, para un caso con $4 f L / D=0, A / \mathcal{A}=0,735, c_{0}(0) t_{f} / L=3,673$ y $p_{\text {exit }}=p_{0}(0) .146$

5.12 Resultados de una interpolación lineal y una interpolación natural cúbica spline para la velocidad adimensional del aire a lo largo del viento en $t=0,1 t_{f}$, para un caso con $4 f L / D=$ $0, A / \mathcal{A}=0,735, c_{0}(0) t_{f} / L=3,673$ y $p_{\text {exit }}=p_{0}(0) . \ldots \ldots 147$

5.13 Error relativo entre la Ecuación (5.72) y las correspondientes soluciones numéricas para distintos tamaños de la malla, $\Delta x$ (diferentes valores de $I$ ), a lo largo del viento para $t=$ 0,02 , y en las secciones de entrada, intermedia y de salida del viento en función de $t$. . . . . . . . . . . . . . . 148

5.14 Error relativo entre la solución de la Ecuación (5.72) y la correspondiente solución numérica en función del tamaño de la malla, $\Delta x$, para $t=0,02$, en las secciones de entrada, intermedia y de salida del viento. . . . . . . . . . . . . 150

5.15 Error entre la Ecuación (5.75) y la correspondiente solución numérica en función del tamaño de la malla, $\Delta x$, para $t=$ 0,04 , en las secciones de entrada, intermedia y de salida del viento. . . . . . . . . . . . . . . . . . . . . . . . . 151

5.16 Error relativo entre la Ecuación (5.79) y las correspondientes soluciones numéricas obtenidas con distintos tamaños de la malla (diferentes valores de $I$ ), para $t=0,02$ a lo largo del viento, y en las secciones de entrada, intermedia y de salida del viento en función de $t \ldots \ldots$. . . . . . . . . . 153

5.17 Error relativo entre la Ecuación (5.79) y la correspondiente solución numérica en función del tamaño de la malla, $\Delta x$, para $t=0,02$, en las secciones de entrada, intermedia y de salida del viento, utilizando una interpolación natural cúbica spline y una interpolación lineal. . . . . . . . . . . . 154

5.18 Presión adimensional del aire en el cilindro para condiciones de evacuación atmosférica en función del tiempo, utilizando los métodos predictor-corrector de Euler modificado y de Adams-Bashforth-Moulton, y un método de RungeKutta para distintos tamaños de malla, con $A / \mathcal{A}=0,1$, $\mathcal{C}_{0}(0) t_{f} / L=10$ y $4 f L / D=5 \ldots \ldots \ldots \ldots \ldots \ldots \ldots$

5.19 Relación adimensional de la presión en el cilindro en función del tiempo adimensional para evacuación atmosférica, $4 f L / D=0$ y diferentes valores de $A / \mathcal{A}$. a) $c_{0}(0) t_{f} / L=5$.

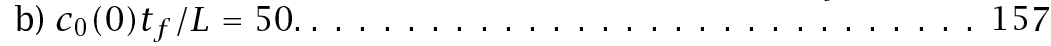


5.20 Relación adimensional de la presión en el cilindro en función del tiempo adimensional para evacuación atmosférica, $4 f L / D=5$ y diferentes valores de $A / \mathcal{A}$. a) $c_{0}(0) t_{f} / L=5$.

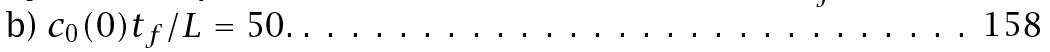

5.21 Relación adimensional de la presión en el cilindro en función del tiempo adimensional para evacuación atmosférica y condiciones quasi-estacionarias, para diferentes valores de $4 f L / D$ y relaciones del área del viento $A / A_{c} \ldots \ldots 160$

5.22 Fracción de masa de aire restante en el cilindro en función de $A / \mathcal{A}$, para evacuación atmosférica, en varios instantes, $t / t_{f}$, y diferentes valores de $c_{0}(0) t_{f} / L$. a) $4 f L / D=0$. b) $4 f L / D=5$. c) $4 f L / D=50 . \ldots \ldots \ldots 16 \ldots \ldots \ldots$

5.23 Fracción de masa de aire restante en el cilindro obtenida con el modelo quasi-estacionario de Bar-Meir et al. (1997) en $t / t_{f}=0,9$, en función de $A / A_{c}$, para evacuación atmosférica y diferentes valores del parámetro $4 f L / D$. . . . . . . 162

5.24 Relación entre la masa de aire en el cilindro en $t / t_{f}=0,9$ predicha por el modelo no estacionario para $c_{0}(0) t_{f} / L=5$ y la predicha por el modelo de Bar-Meir et al. (1997), en función de $A / \mathcal{A}$, para evacuación atmosférica y diferentes valores del parámetro de fricción. . . . . . . . . . . . . . 163

5.25 Relación adimensional de la presión en el cilindro en función del tiempo adimensional para evacuación al vacío, $4 f L / D=$ 0 y diferentes valores de $A / \mathcal{A}$. a) $\mathcal{c}_{0}(0) t_{f} / L=5$. b) $c_{0}(0) t_{f} / L=$

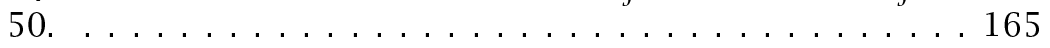

5.26 Relación adimensional de la presión en el cilindro en función del tiempo adimensional para evacuación al vacío, $4 f L / D=$ 5 y diferentes valores de $A / \mathcal{A}$. a) $\mathcal{c}_{0}(0) t_{f} / L=5$. b) $c_{0}(0) t_{f} / L=$

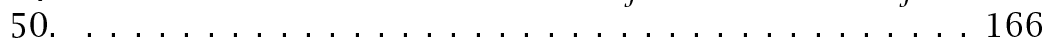

5.27 Fracción de la masa de aire restante en el cilindro para varios instantes adimensionales, $t / t_{f}$, en función de $A / \mathcal{A}$, para evacuación al vacío y diferentes valores de $c_{0}(0) t_{f} / L$. a) $4 f L / D=0$. b) $4 f L / D=5$. c) $4 f L / D=50 \ldots \ldots \ldots \ldots 168$

5.28 Relación entre la masa de aire en el cilindro en $t / t_{f}=0,9$ predicha por el modelo no estacionario para $c_{0}(0) t_{f} / L=10$ y la obtenida considerando condiciones quasi-estacionarias $\left(c_{0}(0) t_{f} / L=500\right)$, en función de $A / \mathcal{A}$, para evacuación al vacío y diferentes valores del parámetro de fricción. . . . . . 169

5.29 Fracción de la masa de aire restante en el cilindro en $t / t_{f}=$ 0,9 , en función de $A / A_{\mathcal{c}}$, para evacuación al vacío, $c_{0}(0) t_{f} / L=$ 500 y diferentes valores del parámetro $4 f L / D \ldots \ldots \ldots 170$ 
5.30 Presión adimensional en el cilindro en función de la relación $A / A_{c}$ predicha por el modelo numérico para $c_{0}(0) t_{f} / L=$ 500 y la correspondiente al modelo de Bar-Meir et al. (1996), para diferentes presiones de vacío y $t / t_{f}=0,9 \ldots \ldots 171$ 



\section{Nomenclatura}

\section{Caracteres latinos}

a Orden de precisión aparente del procedimiento numérico.

A Área ocupada por el aire en la cámara de inyección.

A Área de la sección transversal del viento.

$A_{c} \quad$ Área definida en la Ecuación (5.83).

$\mathcal{A} \quad$ Área definida en la Ecuación (5.22).

c Velocidad de la ola relativa al fluido para una profundidad $h$.

c Velocidad del sonido.

$c_{0} \quad$ Velocidad de la ola relativa al fluido para una profundidad $h_{0}$.

$D \quad$ Diámetro hidráulico del viento.

E Error relativo de la solución numérica.

$\mathcal{E} \quad$ Energía interna del aire en el cilindro.

$f \quad$ Fracción inicial de llenado, $h_{0} / H$.

$f \quad$ Coeficiente de fricción (cuatro veces el factor de fricción).

$f_{\max } \quad$ Fracción inicial de llenado máxima.

$f_{\text {min }} \quad$ Fracción inicial de llenado mínima.

$f^{\prime} \quad$ Fracción inicial de llenado para una cámara de inyección de sección circular.

$f^{*} \quad$ Valor de $f$ definido en las Ecuaciones (2.32) ó (2.49).

$\mathcal{F} \quad$ Parámetro definido en la Ecuación (5.14).

$g \quad$ Aceleración de la gravedad.

$h \quad$ Altura de la superficie libre del metal fundido en la cámara de inyección.

$h_{0} \quad$ Profundidad inicial del metal fundido en la cámara de inyección.

$H \quad$ Altura de la cámara de inyección.

xxix 
Número de nodos en la malla de diferencias finitas a lo largo del viento.

I Número de intervalos en la malla de diferencias finitas a lo largo de la cámara de inyección.

$J \quad$ Número de intervalos de tiempo en el procedimiento numérico de diferencias finitas.

$\ell \quad$ Longitud en la ley de aceleración de la Ecuación (2.61).

$L \quad$ Longitud de la cámara de inyección.

$L \quad$ Longitud equivalente del viento.

$m \quad$ Masa de aire en el cilindro.

$\dot{m} \quad$ Gasto másico de aire a través del viento.

M Número de Mach.

$M_{\text {ent }} \quad$ Número de Mach en la sección de entrada al viento.

$M_{\text {exit }} \quad$ Número de Mach en la sección de salida del viento.

$M_{\max } \quad$ Número de Mach máximo en la sección de entrada al viento para un flujo de Fanno.

$n \quad$ Parámetro en la Ecuación (2.42).

$p \quad$ Presión.

$p_{\text {exit }} \quad$ Presión a la salida del viento.

$q \quad$ Velocidad de transferencia de calor por unidad de flujo de masa.

$\dot{Q} \quad$ Flujo de calor en la Ecuación (5.7).

$t \quad$ Tiempo.

$t_{c} \quad$ Tiempo de formación de la discontinuidad.

$t_{f} \quad$ Tiempo de llenado de la cámara de inyección.

$t_{f} \quad$ Tiempo de llenado del cilindro.

$t_{H} \quad$ Tiempo en el que el metal fundido alcanza el techo de la cámara de inyección.

$t_{\eta} \quad$ Tiempo en el que el metal fundido alcanza una altura $\eta$ en la superficie del pistón.

$t_{1} \quad$ Tiempo en las Ecuaciones (2.8) y (2.9).

$T$ Temperatura.

$u \quad$ Componente horizontal de la velocidad del metal fundido en la cámara de inyección.

$u \quad$ Velocidad del aire en el viento.

$u_{H} \quad$ Velocidad crítica del pistón.

$u_{p} \quad$ Velocidad del pistón.

$u_{\eta} \quad$ Velocidad del pistón en $t=t_{\eta}$.

$v \quad$ Componente vertical de la velocidad del metal fundido en la cámara de inyección.

$V \quad$ Volumen de aire en el cilindro.

$x, y \quad$ Coordenadas horizontal y vertical en la cámara de inyección.

$x_{c} \quad$ Coordenada $x$ de la sección donde se forma la discontinuidad.

$x_{T} \quad$ Coordenada $x$ de la posición de la parte superior del frente de ola en $t_{c}$. 
$X \quad$ Coordenada $x$ de la posición de la superficie del pistón.

$X_{p} \quad$ Distancia inicial entre la superficie del pistón y el extremo del orificio de colada.

\section{Caracteres griegos}

$\alpha, \beta \quad$ Parámetros en la ley exponencial de la Ecuación (2.16).

$\eta \quad$ Altura de la superficie libre en la superficie del pistón.

$\gamma \quad$ Parámetro de la ley potencial de la Ecuación (2.42).

$\gamma \quad$ Relación de calores específicos.

$\lambda \quad$ Pendiente de las líneas características.

$\mu \quad$ Viscosidad del metal fundido.

$\rho \quad$ Densidad del aire o del metal fundido.

$\xi \quad$ Parámetro adimensional, $2 c_{0} / 3 \alpha \beta$.

\section{Subíndices}

$0 \quad$ Condiciones en el cilindro.

$1 \quad$ Condiciones en la sección de entrada del viento.

1 Condiciones en la superficie del pistón.

exact Solución exacta.

ext Solución extrapolada.

opt Condiciones que hacen que la ola empiece a romperse en el instante en el que el metal fundido alcanza la entrada hacia la cavidad del molde.

opt $^{\prime} \quad$ Condiciones que hacen que la ola empiece a romperse justo en la pared final de la cámara de inyección.

$s \quad$ Condiciones quasi-estacionarias.

,,+- o Características positiva y negativa, y trayectoria de la partícula fluida.

\section{Superíndices}

Magnitud adimensional.

Puntos iniciales en la malla de diferencias finitas.

C Corrector.

$P \quad$ Predictor.

\section{Abreviaturas}

CFD Computational fluid dynamics.

CFL Criterio de Courant, Friedrichs y Lewy (1928).

FIAP Fundición por inyección a alta presión.

MAC Marker and cell.

VOF Volume of fluid. 



\section{Introducción}

Los procesos de fundición están entre los procesos de fabricación más antiguos que se conocen (se tiene constancia de su uso desde el año 4000 a. C. para fabricar piezas de ornamentación, puntas de flechas, etc.) y aunque desde su origen hasta fechas relativamente recientes su uso ha estado limitado al mundo de los metales, actualmente sigue ampliándose su aplicación a otras familias de materiales.

Los procesos de fundición de metales consisten básicamente en la introducción de un metal fundido en el interior de un molde y la posterior solidificación del metal reproduciendo la geometría de la superficie interna del molde. Estos procesos son, en general, capaces de reproducir geometrías muy complejas, permitiendo también generar cavidades o superficies internas en las piezas de cierta complejidad. Por otra parte, tienen una gran versatilidad, permitiendo fabricar a bajo coste piezas de una gran diversidad de tamaños y formas, que no puede conseguirse con otros procesos de fabricación, y procesar determinados materiales de difícil tratamiento mediante procesos de otros tipos.

Son diversas las tecnologías que hasta la fecha se han desarrollado en el ámbito de los procesos de fundición de metales. Aunque obviamente las distintas tecnologías tienen sus propias características, aplicaciones, ventajas y limitaciones, para garantizar un adecuado nivel de calidad en el producto existen aspectos comunes a todas ellas cuyo conocimiento es indispensable para controlar el proceso. Estos aspectos son los relativos al flujo del metal fundido, la solidificación y la transferencia de calor, que a su vez están influenciados por otros factores tales como el material y el diseño del molde, el material de la pieza que se va a fabricar o la máquina empleada. 
Desde principios del siglo XX se están desarrollando nuevas tecnologías que permiten reducir los tiempos de producción introduciendo a gran velocidad el metal líquido en un molde metálico generalmente refrigerado. Estos procesos, denominados procesos de Fundición por Inyección a Alta Presión (en adelante procesos FIAP), presentan en la actualidad un gran interés en el sector de automoción, en el que compiten diversas aleaciones ligeras de aluminio (A356 y similares). En la actualidad, aproximadamente el $48 \%$ de la producción de los procesos FIAP se destina a la industria de automoción, el $20 \%$ a la construcción, y el resto a la fabricación de utillajes, componentes electrónicos y computadoras, etc. Desde el punto de vista técnico, los procesos FIAP pueden presentar diversas etapas en la ruta de proceso, desde la fusión del material hasta la expedición del producto obtenido. La primera etapa del proceso incluiría las fases de fusión y mantenimiento a la temperatura de colada, inyección del metal fundido, y extracción y desbarbado de la pieza. El postprocesado de la pieza, después de su extracción, puede incluir procesos tales como tratamientos térmicos, mecanizado, ensayos no destructivos, control dimensional y geométrico, etc. (Faura, 1997).

Las previsiones a corto y medio plazo indican que el consumo de las aleaciones de aluminio en el sector de automoción seguirá creciendo. Como es sabido, las aleaciones de aluminio son las únicas aleaciones metálicas cuya utilización ha experimentado un crecimiento importante y sostenido en las dos últimas décadas (Winter, 1994; Auto Strategies International, 1994)*. Algunas de las causas que han impulsado el crecimiento de las aplicaciones de estas aleaciones en el sector de automoción son las relacionadas con su atractivo para satisfacer algunos de los objetivos prioritarios de la industria de automoción y sus consumidores: reducción del consumo energético (del orden de 1 litro de combustible por cada $100 \mathrm{~km}$ al utilizar carrocerías de aluminio), posibilidades de reciclado, seguridad (sistemas de frenado y dirección más seguros), etc. Otras causas que dan lugar a esta situación están relacionadas con el avance producido en las tecnologías de fundición. Hasta épocas recientes los procesos de fundición presentaban grandes dificultades en los aspectos relativos a la automatización y flexibilidad de la producción, por una parte, y en lo referente a la aparición de defectos en los productos obtenidos por otra. El desarrollo de diferentes técnicas computacionales ha contribuido notablemente al progreso en el estudio de diferentes fases del ciclo productivo de los procesos de fundición, tales como el diseño y la fabricación de moldes, el llenado del molde, la solidificación, etc., lo cual ha introducido un mayor grado de flexibilidad y una mayor capacidad de

\footnotetext{
*A pesar de la reducción de peso de los automóviles en los últimos 20 años (un 16\%, aproximadamente), las aleaciones de aluminio han incrementado su presencia en los automóviles en un $44 \%$ en peso. Se prevé una utilización media de estas aleaciones de entre 140 y $150 \mathrm{~kg} /$ vehículo para mediados de la próxima década.
} 


\begin{tabular}{|l||c|c|c|c|}
\hline $\begin{array}{l}\text { CARACTERÍSTICAS } \\
\text { PRINCIPALES }\end{array}$ & GRAVEDAD & $\begin{array}{c}\text { BAJA } \\
\text { PRESIÓN }\end{array}$ & $\begin{array}{c}\text { ALTA } \\
\text { PRESIÓN }\end{array}$ & $\begin{array}{c}\text { "SQUEEZE } \\
\text { CASTING" }\end{array}$ \\
\hline \hline Método de llenado & Cuchara & $\begin{array}{c}\text { Presión } \\
\text { neumática }\end{array}$ & $\begin{array}{c}\text { Alta velocidad \& } \\
\text { Presión hidráulica }\end{array}$ & $\begin{array}{c}\text { Relativa baja vel. \& } \\
\text { Alta pres. de inyecc. }\end{array}$ \\
\hline Tiempo de llenado (s) & $10 \div 30$ & $10 \div 30$ & 1 & 10 \\
\hline Presión de operac. (MPa) & 0,1 & $0,1+(0,02 \div 0,05)$ & $10 \div 50$ & $50 \div 100$ \\
\hline $\begin{array}{l}\text { Tiempo de ciclo (min) } \\
\text { (p.e., ruedas aluminio) }\end{array}$ & $5 \div 10$ & $5 \div 10$ & $1 \div 2$ & 2 \\
\hline Temperatura del molde & Alta & Alta & Baja & Baja-Media \\
\hline Precisión dimensional & + & ++ & +++ & +++ \\
\hline Flexibilidad de diseño & +++ & ++ & + & + \\
\hline Productividad & + & ++ & +++ & +++ \\
\hline Calidad del producto & + & ++ & $+/+++++$ & +++ \\
\hline
\end{tabular}

Tabla 1.1: Características de diversos procesos de fundición.

predicción de defectos y, por tanto, de su corrección. Una descripción de algunos de los avances de estas tecnologías en el sector de automoción se pueden encontrar en la referencia Kim y Kim (1991).

Además del elevado ritmo de producción que permiten los procesos FIAP, los aspectos relacionados con la precisión dimensional, el acabado superficial, la capacidad para reproducir detalles (Tabla 1.1) y el bajo coste económico han hecho que estos procesos adquieran una posición importante dentro de los procesos de fabricación en general, y en la industria de automoción en particular (Ostermann et al., 1993). Como resultado de la excelente precisión dimensional (tolerancias lineales en torno al $0,3 \%$ en aleaciones de aluminio) y la calidad superficial de las piezas, la mayoría de éstas no requieren mecanizados adicionales salvo un ligero desbarbado y, en ocasiones, operaciones de taladrado y roscado de orificios. Sin embargo, es importante en cualquier caso analizar la influencia de la precisión dimensional requerida en el producto sobre los costes asociados al proceso. Pueden encontrarse estudios sobre este tema en las referencias Sutherland y Roth (1975); Chase y Greenwood (1988); Lee y Woo (1989); Dong et al. (1994).

Frente a las ventajas mencionadas, los problemas más importantes en los procesos de fundición suelen ser la formación de porosidad en las piezas fabricadas, que será discutido posteriormente con mayor detalle, y la aparición de segregaciones durante la solidificación de aleaciones metálicas. Las segregaciones, o zonas de mayor concentración de elementos aleantes, suponen discontinuidades en la estructura de la pieza fundida que deben ser evitadas. De cualquier modo, este tipo de fenómenos no está dentro del ámbito de estudio de esta tesis, aunque los análisis que se llevarán a cabo podrán servir de soporte para que puedan ser considerados en el futuro. 
Desde el punto de vista físico, en los procesos FIAP intervienen muy diversos fenómenos de naturaleza bien diferenciada y compleja. Para analizar este conjunto de fenómenos se puede admitir una descomposición del problema general utilizando por una parte unos modelos que permitan describir el comportamiento de magnitudes macroscópicas tales como temperatura, velocidad del fluido, tensiones de origen térmico, etc., denominados modelos macroscópicos o macromodelos, y por otra parte unos modelos que permitan determinar magnitudes microscópicas tales como tamaño y velocidad de crecimiento de granos, tipo de estructura, etc., usualmente denominados micromodelos. Lógicamente es necesario tener en cuenta las interacciones entre los macro y micromodelos, si bien en la presente tesis se centrará la atención en los primeros, y en particular en determinados problemas relacionados con los aspectos fluidodinámicos exclusivamente.

Un problema inevitable en los procesos FIAP es el derivado de la mayor tendencia a la aparición de niveles elevados de porosidad en las piezas. En piezas con exigencias de funcionamiento poco severas, la disminución de la calidad por porosidad se ve compensada por las grandes ventajas de los procesos FIAP antes mencionadas. Cuando los requerimientos de funcionamiento son mayores, la reducción a un límite admisible del nivel de porosidad es un objetivo esencial que requiere la utilización de tecnología compleja y el conocimiento y control de los factores que determinan el nivel de porosidad.

\subsection{Mecanismos de formación de porosidad}

Se está realizando actualmente un gran esfuerzo de investigación para superar las limitaciones de los procesos FIAP y hacerlos aún más atractivos. En lo que se refiere al problema de la porosidad en los procesos de fundición en general, los primeros trabajos que se realizaron para tratar de predecir su aparición en piezas fundidas partieron de planteamientos basados únicamente en el estudio de los fenómenos de transferencia de calor durante la solidificación del metal líquido (una revisión de dichos trabajos puede verse en Flemings (1974)). De esta forma se identificaron dos de los parámetros que frecuentemente controlan la aparición de porosidad: el tiempo de solidificación (Chvorinov, 1940) y los gradientes de temperatura durante la solidificación del metal fundido (Pellini, 1953; Niyama et al., 1982).

Los avances en dinámica de fluidos computacional han proporcionado herramientas nuevas y muy potentes para analizar los procesos de llenado del molde y de solidificación y la predicción de porosidad de piezas fundidas. Estas nuevas técnicas de análisis, junto con el avance en el conocimiento de los mecanismos de formación de poros, han permi- 
tido utilizar, desde principios de los años ochenta hasta la actualidad, métodos de análisis cada vez más complejos que resuelven el acoplamiento de los fenómenos de transferencia de calor, fluidodinámicos y de solidificación (Hwang y Stoehr, 1983, 1988; Hansen y Sahm, 1988).

Algunos de los primeros trabajos clásicos sobre porosidad en piezas fundidas pueden encontrarse, por ejemplo, en las referencias Piwonka y Flemings (1966) y Campbell $(1967,1969)$. En otros trabajos más recientes sobre el tema se trata de acoplar las dos causas básicas de formación de porosidad, analizando sus efectos combinados. Dichas causas son la existencia de gases disueltos y atrapados en el metal fundido a la entrada del molde (que no fueron considerados en estudios previos) y la resistencia a la alimentación líquida que se presenta en las zonas en solidificación para compensar la contracción volumétrica que experimenta el metal al solidificarse (Kimio y Pehlke, 1985; Poirier et al., 1987; Wang, 1990).

La porosidad en procesos de fundición puede ser de dos tipos: macroporosidad y microporosidad. Ambos tipos pueden tener su origen en las dos causas básicas que se acaban de mencionar. Obviamente, si el proceso de fundición se realizara con ausencia de gas y con una alimentación adecuada se obtendría una pieza libre de poros. Desafortunadamente, la complejidad del proceso es tal que en la práctica resulta imposible alcanzar las condiciones ideales, particularmente en procesos FIAP.

A nivel "microgeométrico", la porosidad (microporosidad) producida por la contracción volumétrica del metal al solidificarse puede generarse por distintos mecanismos. Los problemas de alimentación líquida ${ }^{\dagger}$ pueden dar lugar a porosidad interna producida por un mecanismo de nucleación interna ${ }^{\ddagger}$ (Ampuero et al., 1991) o de iniciación superficial ${ }^{\S}$ (típica esta última de las aleaciones con grandes rangos de solidificación). En algunos casos, las dificultades de alimentación líquida dan lugar a que se

\footnotetext{
${ }^{\dagger}$ Si existen causas que impiden la alimentación líquida en zonas con déficit volumétrico provocado por la contracción experimentada por el metal al solidificarse, se podría iniciar la aparición de un poro. En piezas de geometría compleja, los obstáculos para la alimentación son mayores, agravándose el problema de formación de porosidad.

${ }^{\ddagger}$ Para aleaciones con pequeños rangos de temperaturas de solidificación, como por ejemplo bronce, Al-Si eutéctico o aluminio puro, si un volumen de líquido quedara aislado sin posibilidad de alimentación externa ni interna, el progresivo déficit volumétrico provocado por la solidificación someterá al líquido a una tensión ejercida por el sólido circundante. Si la presión a la que está sometido el líquido alcanza un cierto valor crítico, se formará un poro. El crecimiento inicial del poro se realizará en milisegundos, y alcanzará un tamaño que dependerá de la tensión acumulada. Una vez iniciada la formación del poro, la solidificación progresiva del metal incrementará las tensiones internas que favorecerán su crecimiento. Esta clase de poros se concentra cerca del centro de la pieza fundida. La presencia de partículas extrañas favorece la aparición de porosidad.

§Si la alimentación líquida interior es insuficiente, la contracción volumétrica durante la solidificación hará que la presión hidrostática interna pueda disminuir hasta límites tales que se llegue a producir la succión de líquido fundido desde las zonas superficiales de la pieza que aún no se hayan solidificado. Esta succión de líquido puede arrastrar con él gas exterior que formará un microporo en forma de microcanal con geometría compleja.
} 
produzca una porosidad externa (en la superficie de la pieza) como consecuencia de un mecanismo de alimentación sólida desde el exterior de la pieza. Otro tipo de microporosidad, que suele estar distribuida uniformemente en todo el conjunto de la fundición, es la debida a la precipitación de los gases disueltos en el líquido metálico durante la solidificación".

Algunos tipos de aleaciones, bajo determinadas condiciones de proceso, son particularmente susceptibles a la formación de poros internos que nuclean creciendo rápidamente a lo largo de las capas paralelas a las posiciones que supuestamente ocupan las isotermas durante la solidificación del metal líquido. Esto se favorece en aleaciones con grandes rangos de temperaturas de solidificación en procesos con bajos gradientes de temperatura, tales como los que intervienen en la fundición invertida, la fundición en yeso o la fundición en arena. Estos efectos no son relevantes, por tanto, en procesos FIAP.

A nivel "macrogeométrico" la porosidad (macroporosidad) producida en fundición por gravedad por la contracción volumétrica durante la solidificación adquiere la forma de cono o embudo en la zona del molde destinada a compensar el déficit volumétrico del metal al solidificarse. Si este problema se presenta en regiones aisladas en el interior de la pieza, se generará un macroporo interior a la pieza fundida. En otros casos, la formación de porosidad se debe a que, durante la etapa de llenado, se puede provocar el desprendimiento de gases contenidos en el molde al entrar en contacto con éste el metal fundido. Este problema es típico de los moldes de arena, susceptibles de contener grandes porcentajes de humedad y gases, pero no ocurre en procesos FIAP en los que el molde es metálico.

Además de los mecanismos de generación de porosidad que se acaban de describir brevemente, debe citarse el que tiene lugar como consecuencia de la posible existencia de aire que queda atrapado en el interior del metal fundido durante el proceso de llenado del molde. Este mecanismo es particularmente relevante en procesos FIAP, en los que las especiales características del flujo en la cámara de inyección y la turbulencia en el flujo de metal fundido a la entrada del molde pueden producir la ingestión de una elevada masa de aire, lo que daría lugar a altos niveles de formación de burbujas. Esto se ve agravado por los pequeños tiempos de solidificación característicos de estos procesos (el molde es metálico y generalmente refrigerado) y por la dificultad que los moldes metálicos tienen para evacuar los gases. Koster y Goehring (1941) analizaron el

\footnotetext{
"Cuando el metal se solidifica, la solubilidad de los posibles gases disueltos en el líquido disminuye, precipitando en las zonas interdendríticas (microporosidad). El tamaño habitual de este tipo de poros está en un rango entre 0,01 y 0,5 mm de diámetro. $\mathrm{Al}$ ser tan pequeño su tamaño, cuando la pieza es mecanizada resultan imperceptibles a simple vista. Este tipo de poros, que presentan una distribución uniforme en todo el conjunto de la fundición, se reduce por la presencia de impurezas de gran actividad química en el metal tales como óxidos, sulfuros, fosfuros, etc.
} 
proceso de llenado por inyección observándolo en moldes transparentes. Otros trabajos experimentales más recientes han demostrado la importancia que las condiciones del flujo a la entrada del molde tienen sobre la formación de burbujas de gas atrapado (Hirt, 1991). Lindberg et al. (1991) han comprobado mediante una cámara de alta velocidad que, bajo ciertas condiciones, se puede llegar a interrumpir la continuidad del flujo en la sección de entrada del molde debido a la expansión del aire a la salida de ésta. Ohnaka (1993) ha realizado una revisión de las investigaciones en curso sobre la fluidomecánica del proceso de llenado de un molde, la generación de porosidad y la macrosegregación. La importancia de este mecanismo de generación de porosidad debido al aire atrapado durante el llenado es mucho menor si la velocidad de elevación de las burbujas de gas producidas en la entrada del metal fundido al molde es suficientemente rápida, de forma que el tiempo característico de ascenso de las burbujas es pequeño frente al de solidificación. Esta circunstancia no se da en procesos de fundición a alta presión, y menos aún cuando se trata de piezas con paredes delgadas que se solidifiquen en un tiempo característico suficientemente pequeño en comparación con el tiempo de ascenso de las burbujas. El tamaño de los poros formados varía generalmente entre 1 y $5 \mathrm{~mm}$ de diámetro. Este tipo de poros es fácil de identificar ya que, por su tamaño, cuando la pieza es mecanizada son observables a simple vista. Su distribución en la pieza está lejos de ser uniforme, concentrándose principalmente cerca de las secciones de entrada del metal fundido al molde y en las zonas más elevadas de la pieza, pudiendo quedar el resto libre de poros.

De lo anterior se desprende que existen muy diversos mecanismos que dan lugar a la formación de porosidad. En la presente tesis el estudio se centrará en el último mecanismo descrito, que tiene su origen en la ingestión de gas a través de la superficie libre del metal fundido debido a efectos fluidomecánicos que tienen lugar durante el proceso de llenado del molde. Como ya se ha comentado, este mecanismo es muy importante en procesos FIAP, y de hecho la generación de porosidad a que da lugar limita actualmente la aplicación de la tecnología de inyección a alta presión cuando se trata de fabricar piezas que deben satisfacer requerimientos de funcionamiento estrictos.

\subsection{Tecnología de los procesos FIAP}

El elevado coste del equipo necesario en los procesos FIAP, en especial el de los moldes que puede llegar a suponer aproximadamente el 10\% del coste total de producción, hace que estos procesos se puedan usar solamente para grandes volúmenes de producción. Los altos ritmos de producción permiten conseguir bajos costes de fabricación, que pueden 
llegar a reducirse aún más mediante la automatización y el control del proceso.

Los materiales más empleados para fabricar piezas mediante estos procesos abarcan desde las aleaciones de bajo y medio punto de fusión, como las de estaño, plomo, zinc, aluminio o magnesio, hasta incluso aleaciones de hierro, tales como los aceros inoxidables. Para obtener piezas de aleaciones de bajo punto de fusión suelen emplearse máquinas de cámara caliente, en las que el horno forma parte del sistema de inyección. En la Figura 1.1 se muestra un esquema típico de una máquina de inyección con cámara caliente. No obstante, estas máquinas no se pueden

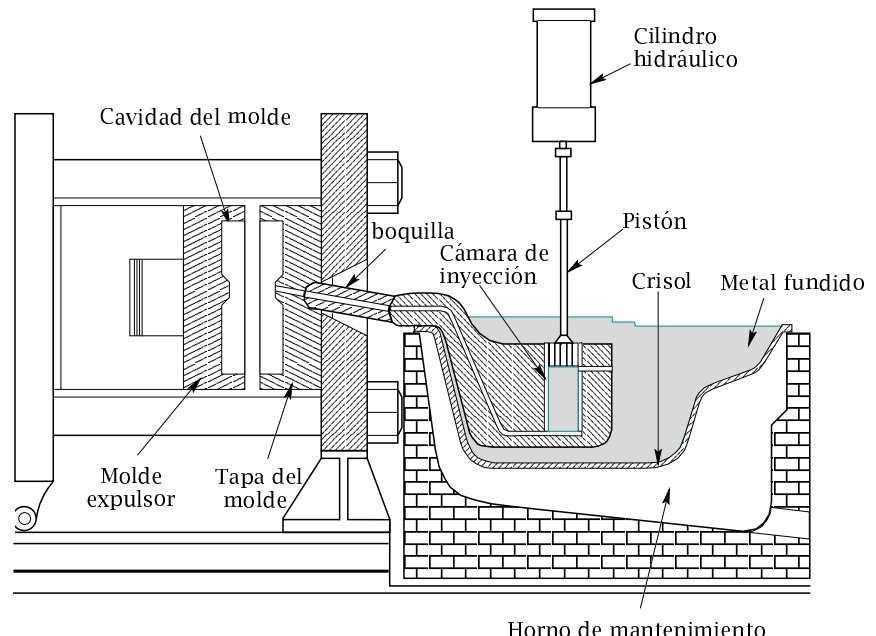

Figura 1.1: Esquema de una máquina de fundición por inyección a alta presión con cámara caliente (basado en Kalpakjian (1995)).

utilizar para fabricar piezas con materiales como las aleaciones de aluminio, cobre o magnesio ya que estos materiales, por encima de su punto de fusión, tienden a deteriorar los elementos férreos que componen el sistema de inyección. En estos casos se emplean máquinas de cámara fría en las que la fusión del metal se realiza fuera del sistema de inyección. Este tipo de máquinas pueden presentar la cámara de inyección en posición vertical u horizontal. Las máquinas con cámara horizontal, para las que el estudio de la fase de inyección que se lleva a cabo en esta tesis tiene mayor interés y cuyo esquema se muestra en la Figura 1.2, permiten ejercer presiones más altas sobre el metal con tiempos de fabricación menores que las máquinas con cámara vertical. En la tecnología de cámara fría horizontal se pueden alcanzar presiones y velocidades de 
inyección del orden de $1000 \mathrm{~kg} / \mathrm{cm}^{2}$ y $6 \mathrm{~m} / \mathrm{s}$ respectivamente, existiendo en el mercado máquinas de hasta 5000 Toneladas de resistencia al cierre del molde.

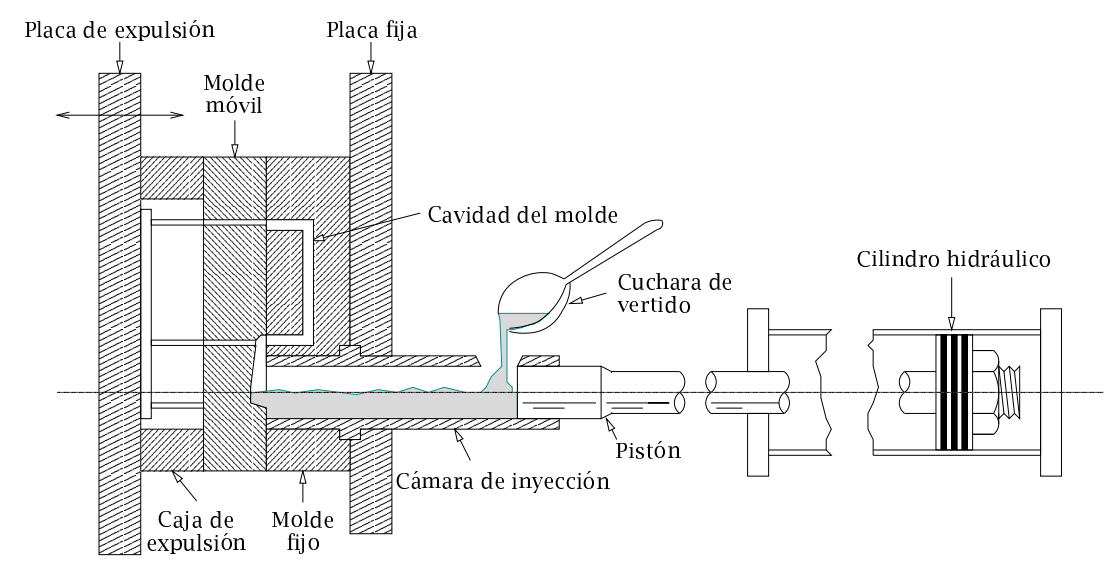

Figura 1.2: Esquema de una máquina de fundición por inyección a alta presión con cámara fría horizontal (basado en Kalpakjian (1995)).

Se ha mencionado que uno de los problemas más importantes que se presentan en estos procesos es la formación de porosidad en las piezas fabricadas. La mayoría de las tecnologías que se han desarrollado en los últimos años están orientadas a minimizar su presencia y sus efectos ("squeeze casting", "pore free", "vacuum casting", etc.). La porosidad influye especialmente en las propiedades mecánicas de las piezas fabricadas, en particular en la ductilidad y resistencia a impactos, por lo que estos procesos raramente se emplean para obtener piezas sometidas a estados de carga críticos. La capacidad de los productos de fundición para ser sometidos a procesos posteriores de tratamiento térmico y soldadura es un indicador de la calidad del proceso, ya que la elevada temperatura requerida para el tratamiento térmico o soldadura puede provocar que las piezas con altos niveles de porosidad lleguen a deformarse debido a la expansión de las posibles burbujas de gas atrapadas en el metal.

\subsubsection{Sistema de inyección}

Durante el llenado del molde y la solidificación de la pieza, los procesos FIAP se pueden programar en varias etapas, cada una de las cuales podría ser optimizada en cierta medida de forma independiente. Cada una de 
estas etapas se caracteriza por la velocidad del pistón o la presión que el sistema de inyección ejerce sobre el metal.

En la etapa de llenado, la presión debe ser relativamente baja si se quieren reducir los efectos fluidodinámicos que favorecen la aparición de porosidad. Ghomashchi (1995) mostró experimentalmente la relación entre la velocidad de inyección, y por lo tanto la presión de inyección, y la formación de porosidad.

En procesos FIAP con cámaras frías horizontales, el metal fundido es inyectado hacia un molde metálico desde una cámara de inyección horizontal en la que el metal es empujado por un pistón. La cámara se llena parcialmente con un volumen de metal fundido (fracción inicial de llenado), desplazándose a continuación el pistón a lo largo de la longitud de la cámara (carrera del pistón), que normalmente es fija. En el proceso de inyección, el aire inicial contenido en el molde y en la cámara de inyección puede ser atrapado en el metal fundido en forma de pequeñas burbujas que causarán porosidad cuando el metal se solidifique. Para minimizar el atrapamiento de aire, el proceso de inyección de metal fundido se suele dividir en una fase lenta, en la que el pistón empuja al metal fundido hasta que la cámara de inyección esté completamente llena, y una fase rápida, en la que el pistón empuja al metal a gran velocidad para llenar la cavidad del molde. En la Figura 1.3 se pueden ver esquemáticamente las dos fases del proceso mencionadas. Smith y Wallace (1963) estudiaron experimentalmente, con agua y metales de bajo punto de fusión, los mecanismos de atrapamiento de aire durante las dos fases del proceso de inyección. Los resultados de sus estudios mostraron que el uso de una velocidad lenta del pistón hasta que la cámara de inyección esté completamente llena reduce apreciablemente el aire atrapado en el metal fundido. Otros autores como Sheptak (1963) o Garber (1982) usaron técnicas similares centrándose exclusivamente en el estudio de la fase lenta del proceso.

En la fase lenta de inyección, generalmente el pistón empuja al metal fundido hasta que alcanza y llena la parte superior de la cámara de inyección, desplazándose a continuación a velocidad constante hasta que la cámara esté completamente llena de metal fundido. Durante el movimiento del pistón se formará una ola. Las observaciones experimentales realizadas por Garber (1982) muestran que existe una velocidad crítica del pistón para que la ola de metal líquido se eleve hasta alcanzar el techo de la cámara de inyección sin romperse. Autores, entre los que se incluyen Garber (1982), Karni (1991) y Tszeng y Chu (1994), propusieron expresiones analíticas (no muy acertadas en los dos primeros casos) para obtener esta velocidad crítica. Kuo y Hwang (1998) utilizaron un modelo matemático para simular el flujo tridimensional de metal fundido en la cámara de inyección con el que determinaron las velocidades críticas correspondientes a varias fracciones iniciales de llenado. Si el pistón al- 


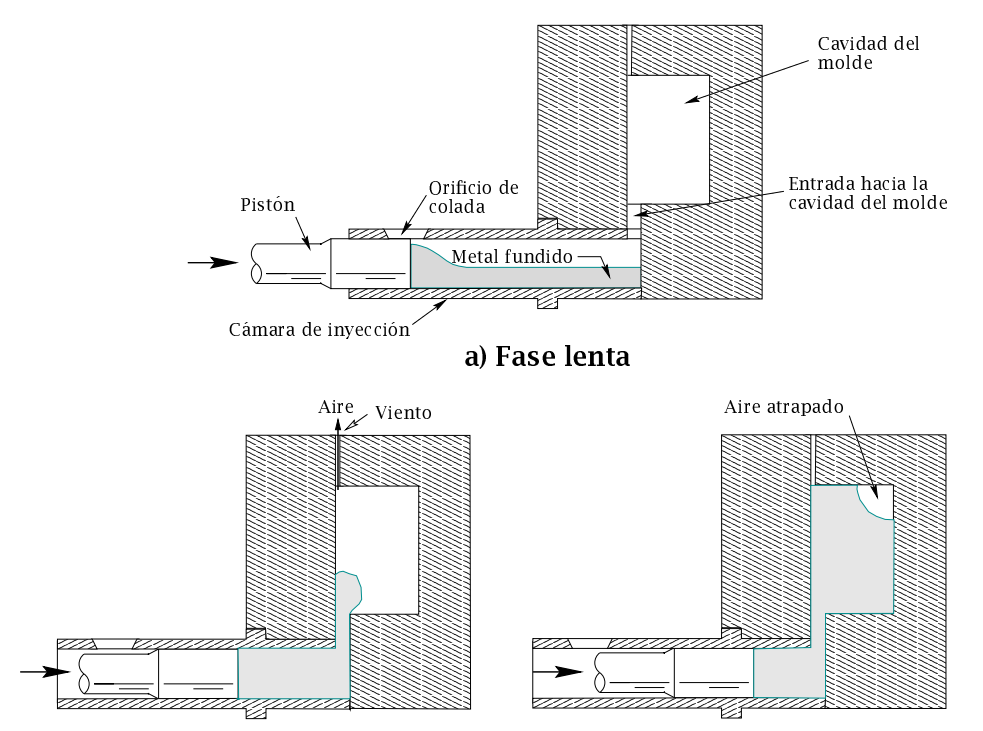

b) Fase rápida

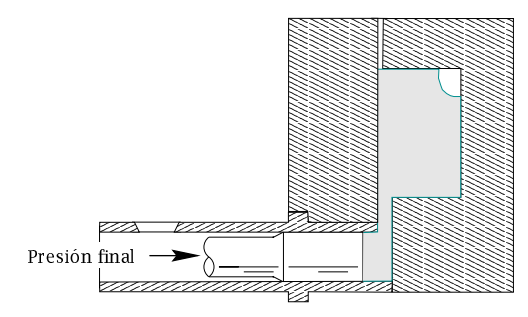

c) Intensificación de la presión

Figura 1.3: Fases de la etapa de inyección en los procesos FIAP con cámaras horizontales. a) Fase lenta. b) Fase rápida. c) Intensificación de la presión. 
canza una velocidad mayor que la crítica, la ola se reflejará en el techo de la cámara de inyección y el frente de ola podría romperse provocando el atrapamiento de aire (Figura 1.4a). Por otro lado, si la velocidad del

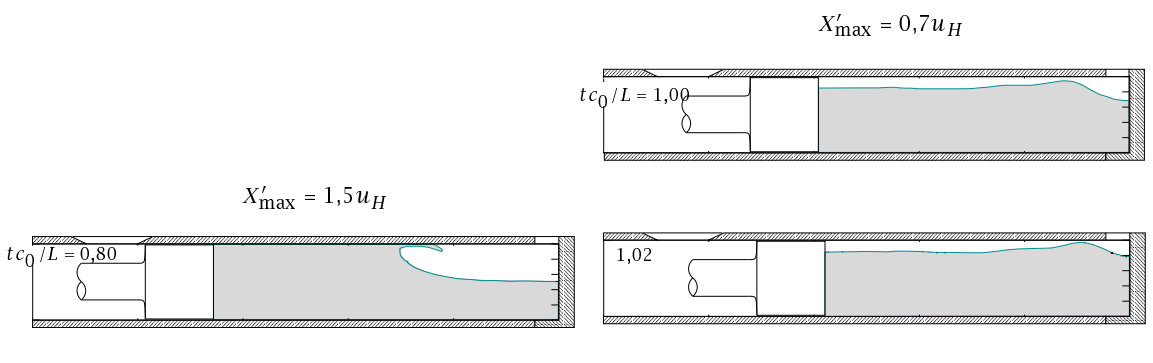

a)

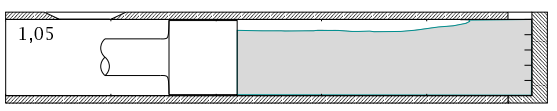

b)

Figura 1.4: Condiciones que tienden a incrementar el atrapamiento de aire: a) velocidad del pistón superior a la velocidad crítica; b) velocidad del pistón inferior a la velocidad crítica.

pistón no alcanza la velocidad crítica, la ola podría reflejarse en la pared final de la cámara y atrapar aire entre el pistón y la parte superior de la cámara de inyección (Figura 1.4b).

Para alcanzar la velocidad crítica, el pistón debe ser acelerado inicialmente. La evolución del perfil de la ola dependerá obviamente de la ley de aceleración del pistón empleada. Aunque existen algunos estudios teóricos y experimentales relativos a la influencia de la aceleración del pistón en el proceso de inyección, en la práctica se pone poco énfasis en la planificación del proceso para controlar la aceleración y el movimiento del pistón hasta alcanzar la velocidad crítica. En Thome y Brevick (1993, 1995), Brevick et al. (1994) y Tszeng y Chu (1994) se pueden encontrar varios estudios teóricos al respecto. Para casos en los que el pistón se mueve con una velocidad que aumenta linealmente con la distancia recorrida, lo que proporciona una aceleración que crece exponencialmente con el tiempo, se han realizado algunos estudios experimentales como los de Karni (1991) y Duran et al. (1991). La razón de esta elección se debe a que en muchas máquinas de fundición por inyección a alta presión los perfiles de velocidad del pistón se suelen programar especificando la velocidad en función de la posición del pistón. La compañía Bühler 
Brothers (1974) desarrolló un sistema de inyección de este tipo llamado "Parashot". Con esta ley de movimiento, Duran et al. (1991) observaron que, para una geometría y una fracción inicial de llenado de la cámara de inyección dadas, existe una aceleración óptima que minimiza el volumen de aire atrapado. Tszeng y Chu (1994) desarrollaron un modelo matemático para estudiar la evolución de la ola formada en la cámara de inyección para el mismo tipo de movimiento del pistón y con las mismas condiciones usadas en los experimentos de Duran et al. (1991). Analizaron los perfiles del frente de la ola y obtuvieron una aceleración óptima del pistón imponiendo como condición que la ola empiece a romperse cuando la parte superior del frente de ola llegue al final de la cámara de inyección. Encontraron que la aceleración óptima obtenida de esta forma se aproximaba bastante bien a las mediciones experimentales realizadas por Duran et al. (1991).

Autores como Lindsey y Wallace (1972) fueron los primeros que realizaron ensayos en máquinas de inyección con cámaras frías horizontales y aleaciones de aluminio, centrándose, aunque parcialmente, en la fase lenta del proceso. Emplearon procedimientos metalográficos y un método tradicional cuantitativo (método de Arquímedes) para estimar los niveles de porosidad interna en piezas fabricadas de Aluminio A380. Con este último método, los niveles de porosidad debidos, principalmente, al atrapamiento de aire y a la contracción volumétrica que experimenta el metal cuando solidifica, se cuantifican a partir de la densidad de las piezas determinada mediante una balanza hidrostática. No obstante, este método no siempre puede ser aplicado debido a que, en ocasiones, las cavidades existentes en la superficie de piezas de geometría de cierta complejidad pueden impedir una determinación suficientemente precisa de la porosidad. Los resultados experimentales obtenidos por Lindsey y Wallace (1972) indican que la fracción inicial de llenado tiene una influencia significativa en la calidad de las piezas fabricadas. Observaron la escasa influencia sobre la calidad de las piezas fabricadas del lubricante que se utiliza, entre otras razones, para evitar que el metal de la pieza se suelde a la superficie de la cavidad del molde, facilitar el desplazamiento de las partes móviles del molde o reducir el desgaste del pistón y de la cámara de inyección. Por otro lado, Brevick et al. (1994) también estudiaron experimentalmente la fase lenta, cuantificando la cantidad de aire atrapado en la cámara de inyección con un método bastante preciso (método de fusión al vacío), con el que se puede medir la cantidad de gas liberado por una pieza tras su fusión en un horno de vacío. Con este método encontraron que, para un grupo de piezas de una aleación de aluminio del tipo A380, aproximadamente el 30\% de la cantidad total de aire atrapado podría ser atribuida a la fase lenta del proceso, lo que pone de manifiesto la gran influencia de esta fase en la formación de porosidad.

Una vez que el molde está completamente lleno, se eleva la presión 
que el pistón ejerce sobre el metal y se mantiene durante la solidificación de la pieza (Figura 1.3c). Esta presión, junto con los datos geométricos de la pieza a fabricar, determina la fuerza necesaria para mantener el molde (formado por varias partes que se acoplan) completamente cerrado, y está, obviamente, limitada por la capacidad del sistema de inyección que se esté empleando. Durante esta etapa, el aumento de la presión ejercida sobre el metal favorece la reducción de la porosidad en la pieza debido a la disminución del volumen que ocupa el aire atrapado. En la Figura 1.5 se representa un diagrama típico en el que se puede ver la evolución de la posición del pistón y la presión del sistema de inyección durante el llenado del molde.

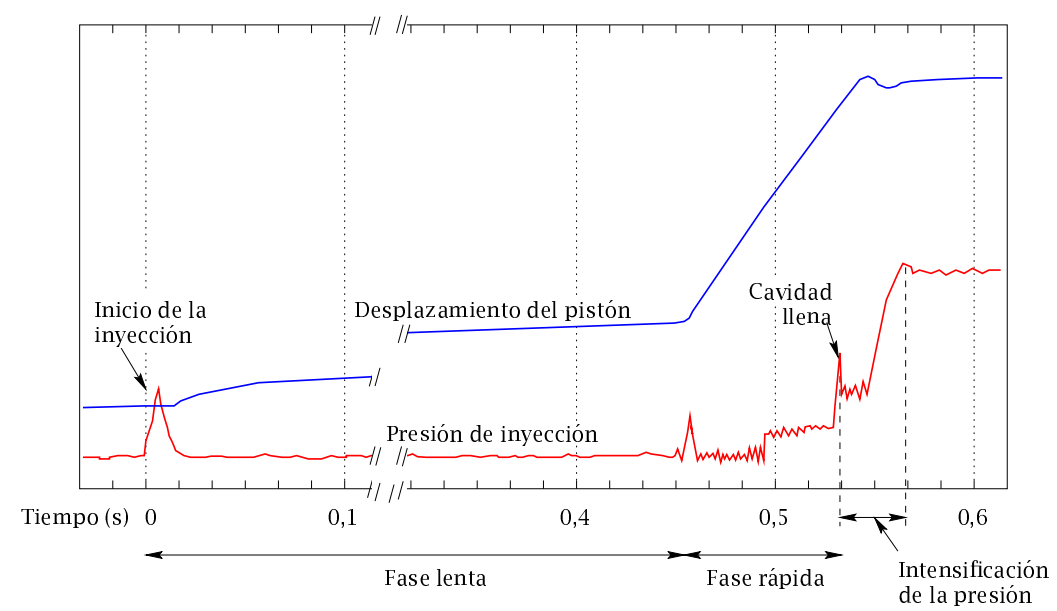

Figura 1.5: Diagrama típico de la presión del sistema de inyección y el desplazamiento del pistón durante el llenado del molde (basado en Street (1977)).

La presión ejercida sobre el metal tiene por tanto efectos contrapuestos sobre la porosidad dependiendo de la etapa del proceso en la que esté actuando. El aumento de la presión dinámica durante el llenado del molde tiende a incrementar los niveles de porosidad en la pieza fundida, debido al aumento de la ingestión de aire, mientras que incrementos de la presión estática durante la solidificación de la pieza tienden a reducirlos. Como ejemplo, Lismont et al. (1990) mostraron cómo aumentos del orden de 1 MPa de la presión estática en la etapa de solidificación redujeron el nivel de porosidad hasta en un $90 \%$ en el proceso considerado.

Una técnica con la que se trata de mantener una presión adecuada en 
cada una de las distintas etapas es el proceso de "squeeze casting" (Doric y Verma, 1988), que combina una muy baja presión durante el llenado del molde (baja velocidad) con una muy alta presión mantenida durante la solidificación de la pieza (puede ser de hasta $140 \mathrm{MPa}$ ), obteniéndose generalmente piezas fundidas libres de poros y con muy buenas características mecánicas. Las características generales de estos procesos se resumen en la última columna de la Tabla 1.1. Puede observarse que su desventaja principal frente a los procesos FIAP es el elevado tiempo típico de llenado requerido, lo que puede ser un factor importante en algunos casos.

\subsubsection{Sistema de evacuación de aire}

El aire inicial que ocupa la cavidad del molde y la parte de la cámara de inyección que no está ocupada por el metal fundido, debe ser evacuado para evitar que quede atrapado en la pieza en forma de poros. Normalmente, en estos procesos el aire es evacuado desde la cavidad del molde hacia el medio ambiente a través de unos conductos de pequeña sección llamados vientos. Para que la evacuación sea óptima, los vientos deben situarse en las zonas del molde que se llenan en último lugar, lo cual es imposible en numerosas ocasiones, lo que hace que en determinadas circunstancias pueda existir aire no evacuado que quedará atrapado y contribuirá a la formación de poros. Es importante indicar que el diseño y la localización de los vientos debe, por un lado, reducir la masa de gas residual en la cavidad del molde al final del proceso de inyección, y por otro, minimizar los trabajos de acabado posteriores y asegurar que el metal fundido se solidifique dentro del molde.

La importancia de disponer de un sistema de evacuación de aire apropiado es mayor conforme el tamaño de la pieza aumenta. Con frecuencia, las piezas pequeñas no precisan de vientos, ya que la evacuación de aire producida a través de la superficie de unión de las partes del molde (línea de partición) suele ser suficiente. Además, en piezas relativamente grandes es habitual colocar junto a los vientos pequeños depósitos (rebosaderos) que permiten, entre otras cosas, retener elementos contaminantes como óxidos, escorias, pequeñas burbujas de aire, restos de lubricante, etc., o facilitar una mayor evacuación de calor en zonas donde pueda ser necesario. Barton (1963) recomienda usar varios depósitos de este tipo de pequeño tamaño" convenientemente situados en el molde. Cuando el metal se solidifica, los rebosaderos deben ser expulsados mediante eyectores para impedir que queden pegados en el molde. En la Figura 1.6 se muestra un esquema representativo de un rebosadero y un

\footnotetext{
"Para el aluminio, Barton (1963) sugiere que un tamaño apropiado para estos depósitos podría ser aproximadamente de $22 \mathrm{~mm}$ de ancho, $32 \mathrm{~mm}$ de largo y $8 \mathrm{~mm}$ de espesor $\left(\frac{7}{8} \times 1 \frac{1}{4} \times \frac{5}{16} \mathrm{in}^{3}\right)$.
} 
viento. En la práctica, los sistemas de evacuación de aire están formados

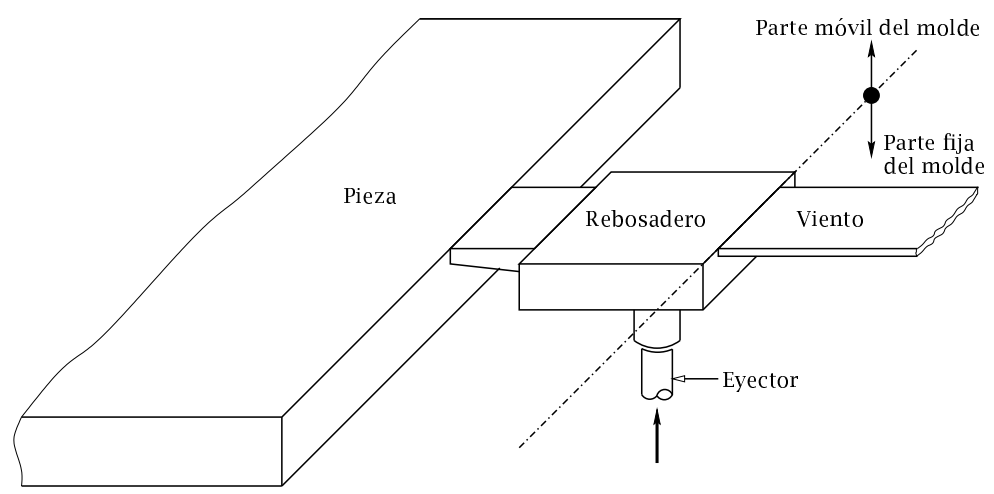

Figura 1.6: Detalle esquemático de un rebosadero y un viento.

por varios vientos de sección rectangular y pequeño espesor para evitar que el metal fundido pueda ser expulsado fuera de la cavidad del molde. Los vientos, que, como se ha dicho, deben situarse en lugares estratégicos para facilitar la completa evacuación del aire, se suelen mecanizar a lo largo de la línea de partición del molde.

En muchos casos, para reducir los efectos del aire atrapado en el metal fundido suele ser suficiente usar máquinas de fundición a alta presión programables que permitan, en primer lugar, la expulsión al ambiente de la mayor parte del aire inicial contenido en el molde y en la cámara de inyección a través de los vientos (evacuación atmosférica), y posteriormente compriman, ejerciendo una gran presión, el aire restante que no ha podido ser evacuado formando pequeñas burbujas en el interior de la pieza. Sin embargo, cuando los requerimientos funcionales o la necesidad de realizar tratamientos térmicos o soldaduras sobre las piezas fabricadas obligan a reducir al máximo los niveles de porosidad, es necesario recurrir a técnicas alternativas como la inyección al vacío. Para conseguir que esta técnica sea eficiente, los vientos deben ser suficientemente grandes ya que de lo contrario el bloqueo sónico del flujo de aire a través del viento impedirá que la reducción de la presión en la cavidad del molde sea suficientemente efectiva. Desde la mitad del siglo XX se han desarrollado muchos sistemas, en especial para máquinas de fundición por inyección a alta presión en cámaras frías, para mantener la acción de la presión de vacío durante el llenado de la cavidad del molde. En la década de los sesenta la "Die Casting Research Foundation" desarrolló un sistema de vientos corrugados y refrigerados que permite la evacuación masiva de aire sin riesgo de que el metal fundido salga fuera del 
molde. Pocos años antes, la compañía suiza F. Hodler \& Cie (Fondarex) desarrolló un sistema de vacío que en la actualidad se está utilizando en muchas máquinas de fundición a presión. Con este sistema, la acción del vacío se activa mediante una válvula que se abre después de que el pistón que empuja al metal fundido en la cámara de inyección haya cerrado el orificio de colada. El aire se evacúa a continuación hasta que la válvula se cierra por la acción de la fuerza hidráulica que el metal ejerce sobre ella. A este sistema, cuyo esquema se representa en la Figura 1.7, se le conoce como "Optivac". Para que el sistema de vacío realice correctamente su

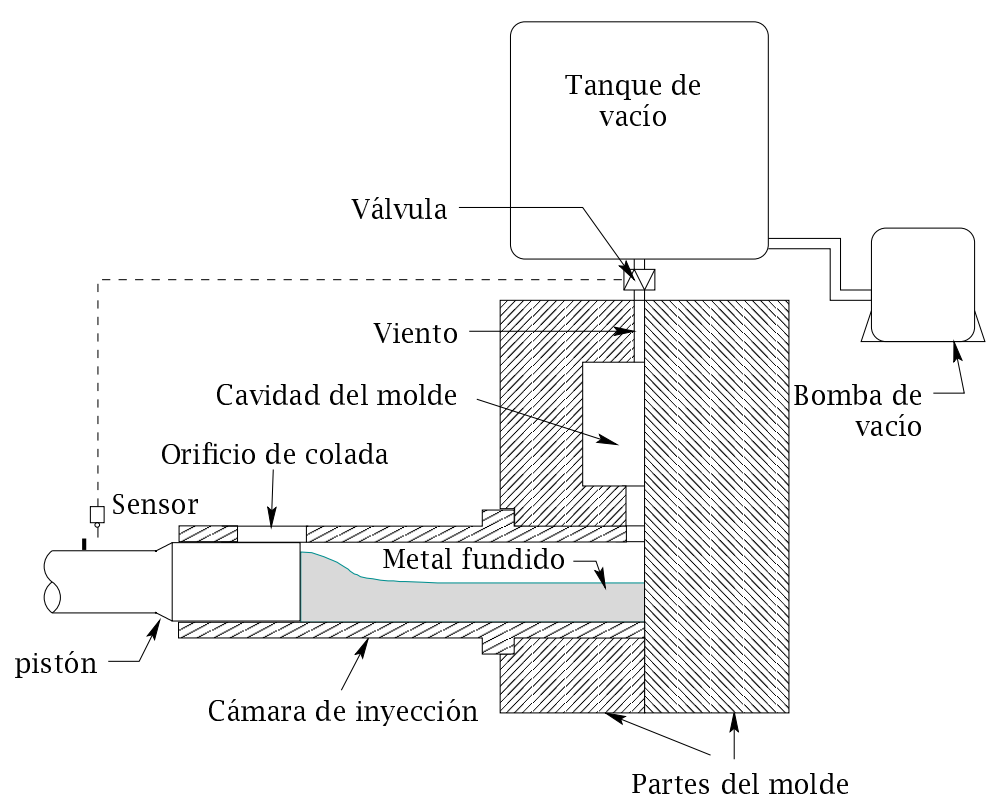

Figura 1.7: Esquema de un sistema de vacío en máquinas de fundición por inyección a presión.

función es conveniente sellar el molde para evitar que el aire exterior, succionado por la diferencia de presión, pueda entrar a través de la línea de partición.

Otro problema que se presenta en los sistemas de evacuación al vacío es el debido al periodo de tiempo tan pequeño en el que se produce el llenado de la cavidad del molde. Para que el aire pueda ser evacuado, en principio sería necesaria una bomba de vacío excesivamente grande y costosa que se usaría únicamente durante el periodo de inyección de metal fundido, no siendo utilizada durante el tiempo restante del ciclo 
productivo de una pieza. Para evitar estos inconvenientes, la bomba generalmente se conecta al viento a través de un tanque de vacío suficientemente grande, de forma que la presión permanezca prácticamente constante en éste durante el proceso de inyección. Bar-Meir et al. (1996) determinaron, por ejemplo, que para que la presión en el tanque de vacío no supere el $25 \%$ de la presión atmosférica durante el llenado de la cavidad del molde, el volumen del tanque debe ser aproximadamente, como mínimo, 7 veces superior al volumen de aire que debe ser evacuado.

Aunque, como ya se ha mencionado, con los sistemas de vacío se obtienen piezas de mejor calidad que con los sistemas de evacuación convencionales, el proceso de fundición es entre un 10\% y un 15\% más caro.

Existe otra técnica alternativa para minimizar la cantidad de aire atrapado en el metal fundido, llamada "pore free", en la que, antes del llenado, se introduce un gas reactivo que tras desplazar el aire inicial del molde reaccionará con el metal líquido formando compuestos como los óxidos** dispersos por todo el metal, lo que reducirá la formación de burbujas de aire atrapado. En principio esta técnica se puede aplicar a todo tipo de aleaciones usando diferentes gases reactivos, aunque el uso de oxígeno para fabricar piezas de aluminio es el caso más extendido. Bar-Meir (1995b) describe detalladamente esta técnica y los casos en los que ofrece mejores resultados.

\subsubsection{1 Área de la sección transversal de los vientos}

Algunos trabajos experimentales, como los de Draper (1967) y Luis y Draper (1967), para aleaciones de bajo punto de fusión, o los de Lindsey y Wallace (1972), para aleaciones de aluminio, realizados en máquinas de fundición por inyección a presión con cámara fría y sistemas de evacuación atmosférica, demuestran que los niveles de porosidad de las piezas fabricadas disminuyen hasta un límite a medida que el área del viento (o el tiempo de llenado) aumenta hacia un valor crítico. Estos resultados demuestran que la correcta determinación de este valor crítico del área de los vientos puede ser crucial para reducir los niveles de porosidad. Para ello, existen algunas recomendaciones de tipo empírico. Por ejemplo, Rearwin (1960) sugiere que, para condiciones de evacuación atmosférica, el área de los vientos debe ser aproximadamente el 50\% del área de la entrada hacia la cavidad del molde. Por otra parte, se han desarrollado diversos modelos teóricos para determinar el valor crítico del área del viento por encima del cual los niveles de porosidad no experimentan una reducción adicional apreciable. La mayoría de estos modelos no tienen en cuenta la resistencia que el viento ofrece al flujo de aire, a pesar de

\footnotetext{
**En el caso del aluminio se obtiene aproximadamente un 0,24\% de óxido de aluminio, lo que no afecta a las propiedades de los productos fabricados.
} 
que la rugosidad superficial y la geometría de los vientos que forman el sistema de evacuación de aire hacen que dicha resistencia pueda tener una influencia importante en el proceso de evacuación. En algunos de estos modelos se supone que el flujo presenta bloqueo sónico en todo momento, como en los propuestos por Sachs (1952) o Veinik (1962), quienes además consideran que la presión en la cavidad del molde es constante. Posteriormente, Veinik (1966) introdujo en su modelo un factor de fricción para cuantificar la resistencia que el viento ofrece al flujo de aire. Bennett (1990) propuso otro modelo en el que, a diferencia de los anteriores, consideró que en el viento no existe bloqueo sónico en ningún momento y que, al igual que en el modelo de Veinik (1966), la presión en la cavidad del molde permanece constante. Todos estos modelos consideran que el proceso de evacuación se puede suponer quasi-estacionario. Karni (1991) desarrolló un modelo en el que se supone que el flujo de aire a través de los vientos es quasi-estacionario y adiabático, considerando además los efectos de fricción. En este modelo, la temperatura del gas en la cavidad del molde se supone constante y se considera la posibilidad de que exista o no bloqueo sónico.

Recientemente, Bar-Meir, Eckert y Goldstein $(1996,1997)$ propusieron un modelo, también quasi-estacionario, en el que se supone que el gas en la cavidad del molde y en la cámara de inyección evoluciona isentrópicamente durante el llenado del molde, y se trata el flujo de gas a través de los vientos como un flujo de Fanno. En los sistemas de evacuación atmosférica, en los que los vientos evacúan el aire directamente al ambiente, la presión del gas en la cavidad aumenta continuamente y en ocasiones el flujo puede llegar al bloqueo sónico. El análisis llevado a cabo por estos autores, para sistemas de evacuación atmosférica, muestra que existe un área del viento crítica, que depende de la geometría y del tiempo de llenado, por debajo de la cual la evacuación de aire es pobre y por encima de la cual la resistencia al flujo de gas es mínima. Para sistemas de evacuación al vacío, el flujo se bloquea casi instantáneamente al principio del proceso de llenado, y el modelo propuesto por Bar-Meir et al. (1996) supone que el flujo está bloqueado a lo largo de todo el proceso de llenado, una hipótesis que es más justificada cuando la presión de vacío es suficientemente pequeña.

Todos estos modelos suponen que el proceso de evacuación de aire es quasi-estacionario. El llenado de la cavidad del molde en los procesos de fundición por inyección a alta presión debe realizarse en un periodo de tiempo muy pequeño (generalmente entre $10 \times 10^{-3}$ y $50 \times 10^{-3}$ s) para evitar que el metal fundido se solidifique en las secciones más estrechas antes de que el molde esté completamente lleno. Por ejemplo, Allsop y Kennedy (1983) recomiendan que para aluminio el tiempo de llenado del molde sea como máximo

$$
40 \times \text { espesor promedio del molde }(\mathrm{mm}) \times 10^{-3} \mathrm{~s} \text {. }
$$


Este proceso de evacuación de aire tan rápido puede producir efectos no estacionarios muy importantes.

\subsection{Aplicación de modelos CFD a procesos de fun- dición}

Para tratar los problemas relacionados con los aspectos fluidodinámicos de los procesos de fundición, se utilizan cada vez más frecuentemente técnicas computacionales. Diversos aspectos relacionados con el tratamiento de la superficie libre en flujos como los que tienen lugar en procesos de fundición constituyen algunas de las dificultades más importantes que son objeto actualmente de intensa investigación en dinámica de fluidos computacional (CFD). Se han desarrollado a lo largo de los años diferentes métodos numéricos para analizar flujos no estacionarios con superficie libre (Tsai y Yue, 1996; Scardovelli y Zaleski, 1999). Ejemplos clásicos de este tipo de esquemas son el MAC ("marker and cell") (Welch et al., 1965) y el VOF ("volume of fluid") (Hirt y Nichols, 1981), que utilizan, respectivamente, partículas marcadoras y una variable volumétrica para el tratamiento de la superficie libre.

A la hora de abordar la resolución numérica de las ecuaciones en derivadas parciales que describen el flujo que tiene lugar durante el proceso de llenado de un molde en el que puede darse un complejo conjunto de fenómenos (véanse, por ejemplo, Szekely y Themelis (1971); Szekely (1979)), que representan modelos físicos más o menos aproximados de dichos fenómenos, se utilizan frecuentemente códigos comerciales de propósito general o específicamente desarrollados para resolver problemas que aparecen en procesos de fundición. La experiencia en el uso de ambos tipos de códigos demuestra que, en ambos casos, su aplicación a un problema concreto, con una determinada configuración geométrica y condiciones iniciales y de contorno adecuadas, puede ser muy compleja, como ocurre en el problema considerado en esta tesis para determinadas condiciones de operación, en las que el flujo es altamente no estacionario en las primeras etapas del proceso de llenado del molde y pueden tener lugar complejos mecanismos de ingestión de aire en el metal líquido, formándose burbujas cuya evolución posterior también debe modelizarse con objeto de predecir el nivel de porosidad al que su presencia da lugar en la pieza fabricada.

La mayoría de los códigos comerciales de interés que se utilizan específicamente en el área de la tecnología de fundición han experimentado recientemente importantes mejoras, incorporando modelos físicos más adecuados y resultando más "amigables" gracias a la provisión de nuevos preprocesadores y postprocesadores que permiten el modelado de geometrías complejas. Por ejemplo, en la Figura 1.8 se representa el modelo 
tridimensional, obtenido con el código A-MESH (EKK, inc.), correspondiente a la tapa del cárter de un motor Diesel fabricado por la empresa Suziki-Santana Motor, S.A. en una máquina de fundición por inyección a presión con cámara fría horizontal, y en la Figura 1.9 se representan los resultados del llenado del molde, en el instante en el que el metal alcanza uno de los vientos (viento 1), obtenidos con el código de elementos finitos Wrafts para diferentes velocidades de inyección del metal fundido (Faura et al., 1997). Entre los recientes desarrollos de estos programas

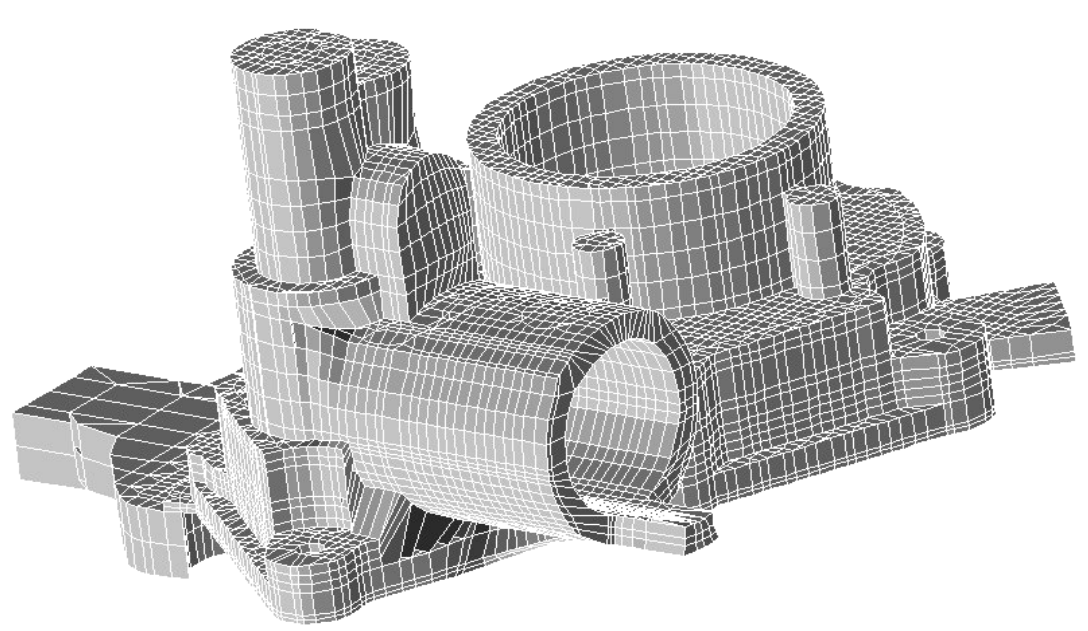

Figura 1.8: Modelado tridimensional de la tapa del cárter de un motor Diesel fabricado en Suzuki-Santana Motor, S.A.

cabe mencionar nuevos esquemas numéricos de discretización, diferentes enfoques para el tratamiento de la superficie libre, alternativas a la hora de utilizar distintos algoritmos para resolución de las ecuaciones discretizadas, avanzados modelos de turbulencia y modelos de fluidos no-newtonianos. Algunos de los nuevos códigos desarrollados admiten el acoplamiento entre modelos de transferencia de calor y flujo fluido, por una parte, y comportamiento termomecánico (tensiones de origen térmico) por otra. 


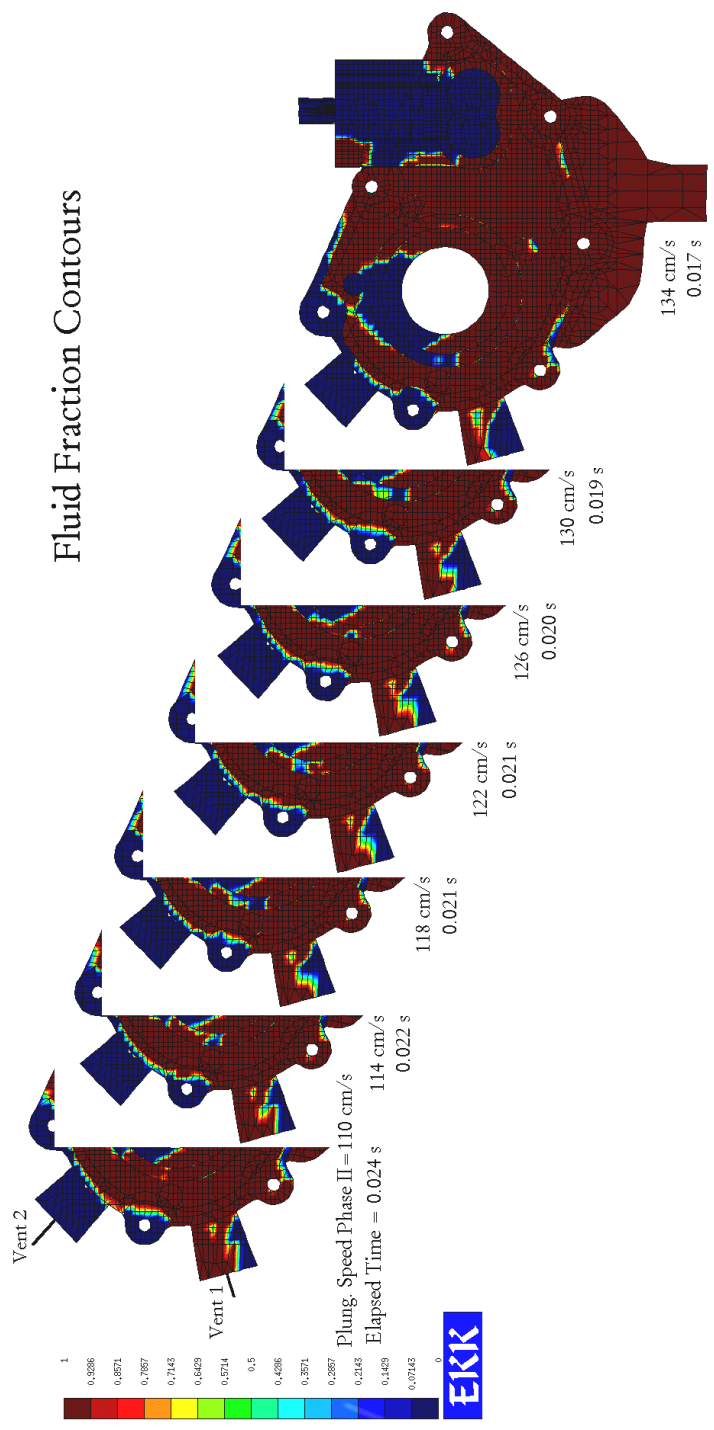

Figura 1.9: Resultados del llenado del molde correspondiente al modelo tridimensional de la Figura 1.8, obtenidos con el código de elementos finitos Wrafts para distintas velocidades de inyección. 


Específicos para problemas de fundición:
WRAFTS-CAP (FE)*
PROCAST (FE)
MAGMASOFT (FV)
"SIMULOR (FV)
RAPID/CAST (FV)
CASTEM (FE)
HICASS (FV)
BACASS (FV)
SOLCAST (FE)
SOLSTAR (EMP)
"CASTS (FE)
AFSolid
De propósito general:
PHOENICS (FV)
FIDAP (FE)
FLUENT (FV)
FLOW3D (FV)
ABAOUS (FE)
DYNA (FE)
ANSYS/FLOTRAN (FE)
NEKTON (FE)
P/THERMAL (FE)
ASTEC (FE)
Modelado sólido y generación de malla:
A-MESH
PATRAN
IDEAS
TRUE GRID
Q-MESH
*FE: Elementos finitos
FV: Volúmenes finitos
EMP: Empírico

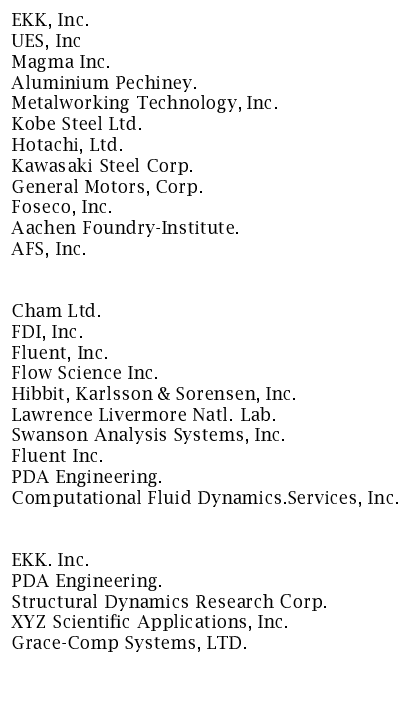

Tabla 1.2: Lista parcial de códigos de fundición y de propósito general usados para simulación de procesos de fundición.

La Tabla 1.2 presenta una lista de códigos frecuentemente utilizados actualmente. Estos códigos pueden incluir:

- representación de formas complejas del molde;

- modelos de flujo fluido transitorio y secuencias de llenado de molde;

- modelo de transferencia de calor por radiación;

- modelos que realizan conjuntamente el análisis de la transferencia de calor y la solidificación;

- modelo de transformaciones en estado sólido-líquido y sólido;

- modelo de tensiones térmicas;

- predicción en la formación de defectos;

- predicción de características microestructurales, y

- predicción de propiedades mecánicas (por ejemplo, dureza y resistencia mecánica).

Ninguno de los modelos actuales integran a la vez todos estos requisitos que se acaban de mencionar, pero se han hecho importantes avances. 
Son de destacar las posibilidades de los códigos en lo que se refiere a la continua actualización de los modelos a la luz de la experiencia operacional, así como el hecho de que tengan una estructura abierta y flexible.

\subsection{Objetivos de la tesis}

Teniendo en cuenta que, según se ha explicado, las causas de atrapamiento de aire en los procesos de fundición por inyección a alta presión se pueden atribuir principalmente a la utilización de condiciones de operación que aumentan los procesos de ingestión de aire durante el tiempo de inyección y al uso de sistemas de evacuación de aire inadecuados, el objetivo principal de esta tesis es estudiar y determinar las características y condiciones de operación durante la fase lenta del sistema de inyección (fracción inicial de llenado, geometría de la cámara de inyección y ley de movimiento del pistón, fundamentalmente) y del sistema de evacuación (geometría de los vientos, localización de los vientos, condiciones de evacuación de aire: atmosférica o al vacío, etc.), que minimicen los fenómenos de atrapamiento de aire y reduzcan en lo posible los tiempos de inyección, lo que permitirá reducir la porosidad en las piezas fabricadas, aumentar el ritmo de producción y evitar posibles solidificaciones prematuras del metal fundido durante el proceso de inyección. Se trata asimismo de determinar los rangos de los parámetros relevantes del proceso que en cualquier caso deben ser evitados para que el proceso de inyección se realice correctamente. La analogía entre las ecuaciones de conservación utilizadas para analizar las condiciones de proceso de ambos sistemas permitirá utilizar en ambos casos técnicas numéricas similares.

En esta tesis no se considerará la posible existencia de fenómenos de solidificación ya que, en condiciones adecuadas de llenado de la cavidad del molde, estos efectos no deben en principio afectar apreciablemente al proceso de inyección estudiado en este trabajo, aunque sí pueden existir condiciones (en particular, por ejemplo, cuando se utilicen aleaciones con determinados rangos de temperatura de solidificación) que requieran un estudio más detallado de dichos efectos. También se supondrá que el metal fundido está en reposo en la cámara de inyección en el instante en el que el pistón inicia el proceso de llenado, aunque si la inyección empieza demasiado pronto el movimiento del flujo provocado por el vertido del metal fundido en la cámara de inyección a través del orificio de colada impedirá que se pueda alcanzar la condición de reposo. Se despreciarán efectos turbulentos y de tensión superficial, aunque pueden existir situaciones, en especial para condiciones alejadas de las óptimas, en las que estos efectos pueden ser importantes. También se despreciarán los procesos de mezcla debidos a las reacciones químicas 
entre el aire y el metal fundido que puedan producirse durante el llenado del molde.

Para analizar el flujo de metal fundido en la cámara de inyección se desarrollará un modelo bidimensional en el que se despreciarán los efectos viscosos y se utilizará la aproximación de aguas poco profundas. En primer lugar el modelo se resolverá analíticamente despreciando los efectos de reflexión del metal contra la pared final de la cámara de inyección, lo que permitirá obtener, para las leyes de movimiento del pistón habitualmente usadas en los procesos FIAP, relaciones adimensionales entre las variables características del problema que optimicen el proceso de inyección. También se determinará mediante este modelo una nueva ley de aceleración del pistón con la que se espera eliminar el aire atrapado en la cámara de inyección minimizando el tiempo de llenado. Posteriormente, se resolverá numéricamente el modelo de aguas poco profundas para considerar los efectos de reflexión, con el que se podrá determinar la influencia de los parámetros que caracterizan la ley de aceleración sobre el volumen de aire atrapado en la cámara de inyección en el instante en el que el metal fundido alcanza la entrada hacia la cavidad del molde. La influencia de los efectos viscosos y no hidrostáticos se estudiará resolviendo las correspondientes ecuaciones de conservación mediante un código de elementos finitos basado en el método VOF para determinar la posición de la superficie libre en cada instante en el que la malla de cálculo se mueve y deforma usando una técnica del tipo LagrangianoEuleriano arbitrario.

Por otro lado, el flujo en los sistemas de evacuación de aire se analizará utilizando un modelo no estacionario en el que el aire se considerará como un gas perfecto y el flujo a través de los vientos se supondrá unidimensional y con fricción. Los resultados de este modelo, para condiciones de evacuación atmosféricas y al vacío, permitirán determinar los rangos habituales de los parámetros de operación en los que los efectos no estacionarios, despreciados en los modelos desarrollados previamente, puedan ser importantes, lo que facilitará el diseño apropiado de los sistemas de evacuación de aire en los procesos FIAP para minimizar la cantidad de aire atrapado.

En el Capítulo 2 se desarrollará el modelo analítico de aguas poco profundas y se aplicará a leyes de aceleración exponencial y potencial en el tiempo, utilizadas habitualmente en los procesos FIAP. Algunos resultados de este modelo para la ley de aceleración exponencial se compararán con resultados experimentales. Finalmente se determinará una nueva ley de aceleración que reduciría a cero el aire atrapado y minimizaría el tiempo de llenado.

En el Capítulo 3 se describirá el procedimiento numérico empleado para resolver el modelo de aguas poco profundas cuando se tienen en cuenta los efectos de reflexión de la ola de metal fundido y se expondrán 
sus resultados para la ley de aceleración exponencial.

El modelo numérico empleado para tener en cuenta los efectos viscosos e hidrostáticos se describirá en el Capítulo 4 y se compararán sus resultados con los del modelo de aguas poco profundas y con resultados experimentales de otros autores.

En el Capítulo 5 se describirá el procedimiento numérico empleado para resolver el modelo no estacionario de evacuación de aire y se expondrán sus resultados para condiciones de evacuación atmosférica y al vacío.

En el Capítulo 6 se resumirán las conclusiones más destacadas obtenidas en los capítulos anteriores y se indicarán posibles extensiones futuras del trabajo desarrollado en esta tesis.

En el Apéndice A se exponen las ecuaciones de conservación simplificadas con la aproximación de aguas poco profundas y se obtendrán, para el flujo en la cámara de inyección y para el flujo unidimensional no estacionario de un gas perfecto, las ecuaciones características y de compatibilidad correspondientes. 


\section{Estudio analítico del movimiento del metal fundido en la cámara de inyección}

En este capítulo se estudia analíticamente el movimiento del metal fundido en el interior de la cámara de inyección durante la fase lenta del proceso de fundición por inyección a alta presión. En este estudio no se consideran los efectos viscosos y el problema se trata bidimensionalmente utilizando la aproximación de aguas poco profundas y la teoría de ondas de amplitud finita. Se consideran dos leyes de movimiento del pistón para las que se analizan en profundidad los posibles perfiles de la superficie libre del metal fundido en función de los parámetros que caracterizan dichas leyes de aceleración, la fracción inicial de llenado de la cámara de inyección y las características geométricas del problema. Se determinan, para distintas situaciones, el lugar y el instante en el que el perfil de la ola llega a ser vertical, es decir, el momento en el que aparece una "discontinuidad" en la superficie libre del metal fundido. En función de la forma del perfil de la ola se discute la selección de los parámetros que permitan reducir la cantidad de aire atrapado. Se tiene en cuenta que para reducir la cantidad de aire atrapado se debe evitar que la ola se rompa dentro de la cámara, y los posibles efectos del aire atrapado debidos a la reflexión de la ola en la pared final de la cámara. Puesto que la 
duración de la fase lenta debe ser lo más pequeña posible con objeto de aumentar la producción e impedir que el metal se solidifique durante el proceso de inyección, parece razonable imponer como condición límite que el frente de la ola deba empezar a romperse justo en la pared final de la cámara. Las predicciones obtenidas de esta forma se comparan con los resultados analíticos de Tszeng y Chu (1994) y los resultados experimentales de Duran et al. (1991). Se demuestra que ninguna de las leyes de aceleración consideradas permite elegir los parámetros de tal forma que se obtenga un perfil de la ola completamente vertical en el instante en el que ésta alcanza la pared final de la cámara de inyección (lo cual evitaría cualquier reflexión parcial de la ola y reduciría a cero la cantidad de aire atrapado en el momento del impacto). Por esta razón se determina una nueva ley de aceleración que eliminaría completamente el aire de la cámara de inyección al final de la fase lenta y minimizaría adicionalmente el tiempo de llenado. También se determinarán, para un amplio rango de los parámetros de aceleración y de la localización del orificio de colada por el que el metal fundido se introduce en la cámara de inyección, los valores límite de la fracción inicial de llenado para que las condiciones de operación sean apropiadas.

\subsection{Modelo analítico}

En la Figura 2.1 se presenta esquemáticamente una máquina de fundición por inyección a alta presión, con cámara de inyección horizontal de longitud $L$ y altura $H$. El problema se considerará bidimensional, con el sistema de coordenadas representado en la Figura 2.2. El fondo de la cámara (en $y=0$ ) se supondrá horizontal y la superficie libre vendrá dada por $y=h(x, t)$. Se supondrá que el valor típico de $h(x, t)$ es mucho más pequeño que la longitud típica horizontal de la ola, por lo que se podrá usar la aproximación de aguas poco profundas (véase el Apéndice A.1). Con esta aproximación, la componente vertical de la aceleración se puede despreciar cuando se compara con la aceleración de la gravedad, $g$, y por lo tanto, si se desprecian los efectos viscosos, la distribución de la presión se puede considerar hidrostática: $p=p_{0}+\rho g(h-y)$. Si la componente horizontal de la velocidad, $u$, es inicialmente independiente de $y$, se puede deducir que $u$ será independiente de $y$ en cualquier instante. Introduciendo $c(x, t)=(g h)^{1 / 2}$, las ecuaciones que describen el problema se pueden escribir de la forma siguiente (Lamb, 1945):

$$
\begin{aligned}
& {\left[\frac{\partial}{\partial t}+(u+c) \frac{\partial}{\partial x}\right](u+2 c)=0,} \\
& {\left[\frac{\partial}{\partial t}+(u-c) \frac{\partial}{\partial x}\right](u-2 c)=0 .}
\end{aligned}
$$




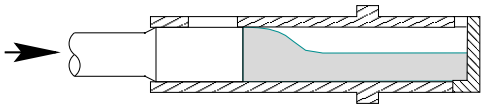

Fase lenta

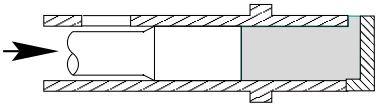

Fase rápida

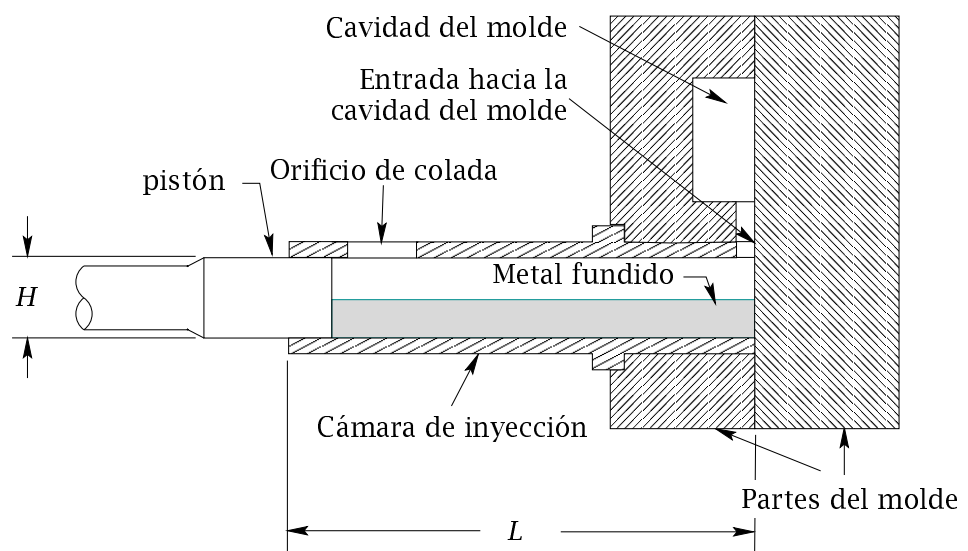

Figura 2.1: Esquema de una máquina de fundición por inyección a alta presión con cámara de inyección horizontal.

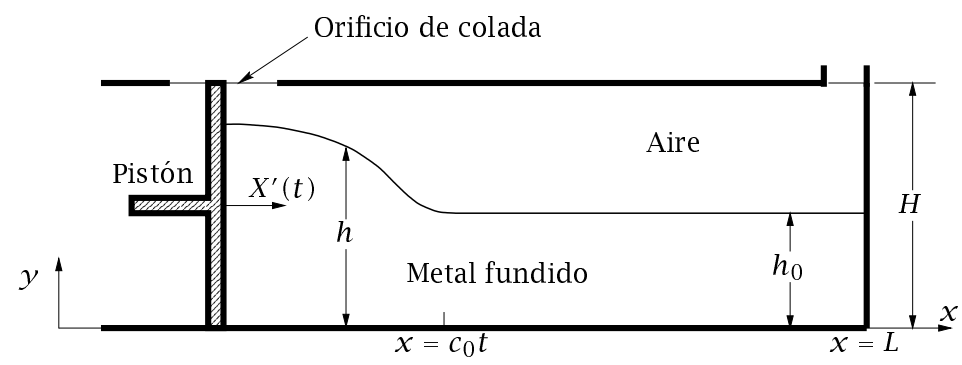

Figura 2.2: Esquema del problema y sistema de coordenadas. 
Usando el método de las características, cuya aplicación a este problema se puede ver detalladamente en el Apéndice A.1, estas ecuaciones se reducen a las condiciones

$$
u \pm 2 c=\text { constante }
$$

a lo largo de las líneas características definidas por

$$
\frac{\mathrm{d} x}{\mathrm{~d} t}=u \pm c
$$

En la Figura 2.3 se ha representado un diagrama con las líneas características del problema.

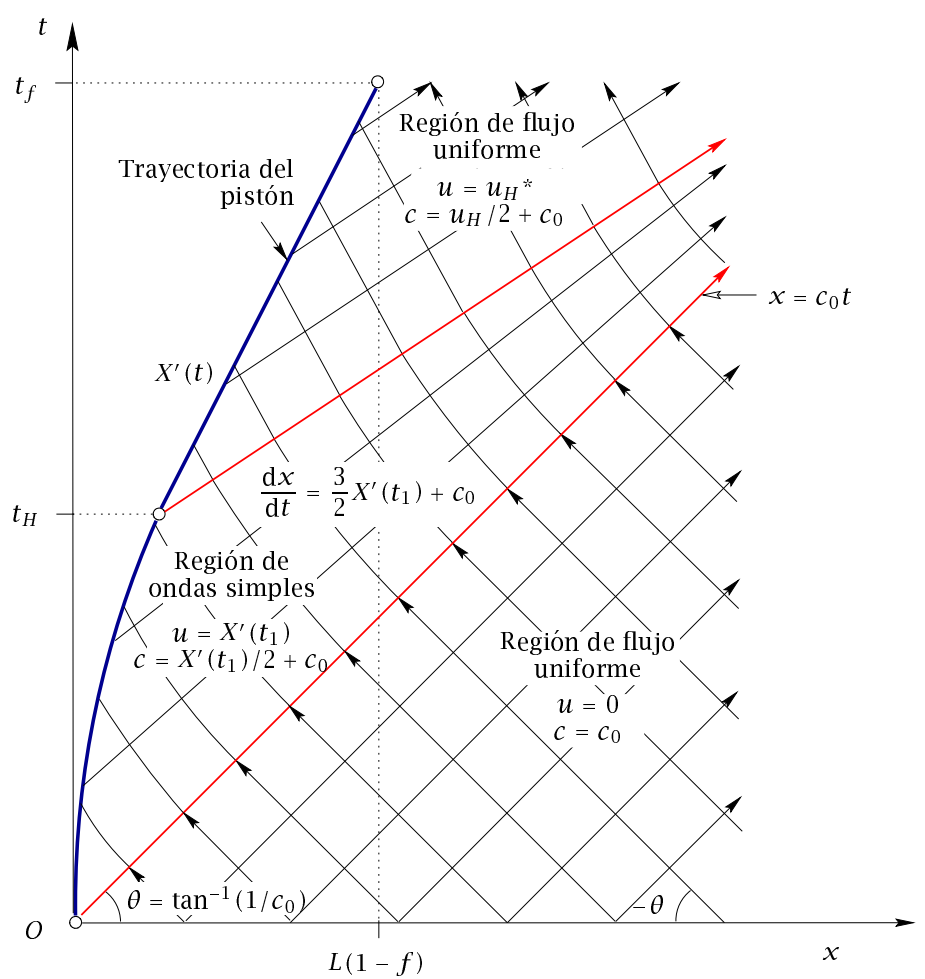

* $u_{H}$ es la velocidad del pistón (velocidad crítica) en el instante $t_{H}$ en el que el metal fundido alcanza el techo de la cámara $(y=H)$ en la superficie del pistón y cesa la aceleración del pistón.

Figura 2.3: Diagrama de las líneas características del problema.

Supongamos que el metal fundido está inicialmente en reposo en la cámara, con una profundidad uniforme $h_{0}$, y que la superficie del pistón 
en contacto con el metal empieza a moverse en $t=0$ en la dirección positiva de $x$ según la ley $x=X(t)$, con $X(0)=0$. Según la Ecuación (2.3), a lo largo de cualquier característica negativa procedente de la región no perturbada en el plano $x t$, donde las propiedades del flujo son uniformes con $u=0$ y $c=c_{0}=\left(g h_{0}\right)^{1 / 2}$, se debe satisfacer la siguiente condición:

$$
c=\frac{u}{2}+c_{0} .
$$

Como $u+2 c$ es constante a lo largo de cada característica positiva, se puede deducir de la Ecuación (2.5) que $u$ es también constante a lo largo de ella. Integrando la característica positiva de la Ecuación (2.4) y usando la Ecuación (2.5) se obtiene

$$
x=\left(\frac{3}{2} u+c_{0}\right) t+f(u)
$$

donde $f(u)$ es una función de la velocidad que puede obtenerse a partir de las condiciones de contorno. Para un instante dado $t_{1}$, la superficie del pistón se encuentra localizada en $x=X\left(t_{1}\right)$ y la velocidad a lo largo de la característica positiva a través del punto $\left(X\left(t_{1}\right), t_{1}\right)$ es, por lo tanto, $u=X^{\prime}\left(t_{1}\right)$. Introduciendo esta condición en la Ecuación (2.6) se obtiene

$$
f(u)=X\left(t_{1}\right)-\left[\frac{3}{2} X^{\prime}\left(t_{1}\right)+c_{0}\right] t_{1},
$$

por lo que la solución en forma paramétrica se puede expresar como

$$
\begin{gathered}
x=X\left(t_{1}\right)+\left[\frac{3}{2} X^{\prime}\left(t_{1}\right)+c_{0}\right]\left(t-t_{1}\right), \\
u=X^{\prime}\left(t_{1}\right) .
\end{gathered}
$$

Estas ecuaciones determinan la velocidad como una función implícita de $x$ y $t$, y mediante la Ecuación (2.5), que puede escribirse

$$
c=c_{0}+\frac{X^{\prime}\left(t_{1}\right)}{2},
$$

se puede obtener el perfil de la ola de metal fundido en cada instante.

Si $h$ y $u$ llegan a ser funciones múltiples de $x$ aparecerá una discontinuidad en el perfil de la ola (el modelo empleado sólo admite soluciones en las que $c$ y $u$ son funciones de $x$ y $t$ ). La localización, $x_{c}$, y el instante, $t_{c}$, en el que se forma dicha discontinuidad se puede determinar a través de las siguientes condiciones simultáneas:

$$
\begin{aligned}
& \left.\frac{\partial x}{\partial t_{1}}\right)_{t}=0 \\
& \left.\frac{\partial^{2} x}{\partial t_{1}^{2}}\right)_{t}=0 .
\end{aligned}
$$


La segunda condición se debe reemplazar por $t_{1}=0$ si la discontinuidad se forma justo en el extremo más avanzado de la ola (en este caso, las primeras características que se cruzan proceden de $t_{1}=0$ y $t_{1}=0^{+}$).

Dado que la sección transversal de la cámara de inyección es normalmente circular y que el flujo es simétrico con respecto al plano vertical que contiene el eje de la cámara, los resultados obtenidos con la hipótesis introducida en esta sección sólo podrán ser aplicados a este plano de simetría. A pesar de esta limitación, el análisis que a continuación se expone se espera que permita tener en cuenta las características esenciales del flujo y determinar las variables óptimas del proceso. En Tszeng y Chu (1994) se puede encontrar una discusión sobre la forma de la superficie libre fuera del plano de simetría en cámaras de inyección de sección transversal circular.

\subsection{Movimiento del pistón con aceleración expo- nencial}

Tszeng y Chu (1994) y, antes que ellos, Duran et al. (1991) en sus estudios experimentales, consideraron la siguiente ley de movimiento del pistón:

$$
x=X(t)=x_{0} \mathrm{e}^{\alpha t},
$$

donde $x_{0}$ es la posición del pistón en $t=0 \mathrm{y} \alpha=X^{\prime}(t) / x$ es una constante que representa la variación de la velocidad del pistón por unidad longitud recorrida. Según esta ley, el pistón tendría una velocidad

$$
X^{\prime}(t)=\alpha x_{0} \mathrm{e}^{\alpha t},
$$

y por lo tanto $X^{\prime}(0)=\alpha x_{0}$ en $t=0$. Para tener en cuenta que el pistón empieza a moverse desde el reposo, se usará la siguiente ley en lugar de la correspondiente a la Ecuación (2.14) ( $\alpha$ y $\beta$ son constantes positivas):

$$
X^{\prime}(t)=\alpha \beta\left(\mathrm{e}^{\alpha t}-1\right),
$$

lo que proporciona la misma aceleración,

$$
X^{\prime \prime}(t)=\alpha^{2} \beta \mathrm{e}^{\alpha t}
$$

que la correspondiente a la Ecuación (2.14) con $x_{0}=\beta$. Integrando la Ecuación (2.15), suponiendo que la localización inicial de la superficie del pistón está en $x=0$, se obtiene

$$
X(t)=\beta\left(\mathrm{e}^{\alpha t}-\alpha t-1\right) .
$$

Sustituyendo las Ecuaciones (2.15) y (2.17) en la Ecuación (2.8), se obtiene

$$
x=\beta\left(\mathrm{e}^{\alpha t_{1}}-\alpha t_{1}-1\right)+\left(\frac{3}{2} \alpha \beta \mathrm{e}^{\alpha t_{1}}-\frac{3}{2} \alpha \beta+c_{0}\right)\left(t-t_{1}\right) .
$$




\subsubsection{Instante y lugar de formación de la discontinuidad}

A continuación se buscará el lugar y el instante en el que se forma la discontinuidad en la ola. Aplicando la condición expresada por la Ecuación (2.11) en la Ecuación (2.18), obtenemos

$$
t_{c}=t_{1}+\frac{2 c_{0}+\alpha \beta\left(\mathrm{e}^{\alpha t_{1}}-1\right)}{3 \alpha^{2} \beta \mathrm{e}^{\alpha t_{1}}},
$$

y de la Ecuación (2.12),

$$
t_{c}=t_{1}+\frac{4}{3 \alpha}
$$

donde $t_{c}$ es el instante crítico en el que $h$ y $u$ se convierten en funciones múltiples de $x$. De las Ecuaciones (2.19) y (2.20), se obtiene

$$
t_{c}=\frac{1}{\alpha}\left[\frac{4}{3}+\ln \left(\xi-\frac{1}{3}\right)\right]
$$

donde

$$
\xi=\frac{2 c_{0}}{3 \alpha \beta}
$$

es el inverso de un número de Froude.

Para $\xi=\frac{4}{3}$, se deduce de las Ecuaciones (2.20) y (2.21) que $t_{1}=0$, y la discontinuidad se formará en el instante

$$
t_{c}=\frac{\xi}{\alpha}=\frac{4}{3 \alpha}
$$

El lugar en el que se forma la discontinuidad se puede obtener sustituyendo $t$ en la Ecuación (2.18) por $t_{c}$ de la Ecuación (2.23) y haciendo $t_{1}=0, \mathrm{y}$ obviamente corresponde al extremo más avanzado de la ola (las perturbaciones a lo largo de la característica que procede de $t_{1}=0$ se propagan con velocidad $c_{0}$ ),

$$
x_{c}=c_{0} t_{c}=\frac{4 c_{0}}{3 \alpha} .
$$

Para $\xi<\frac{4}{3}$, la condición de la Ecuación (2.12), que proporcionaría valores negativos de $t_{1}$, se debe reemplazar por $t_{1}=0$, lo que es equivalente a imponer que $u=0$ en el límite de la zona de metal fundido en reposo, por lo que el instante en el que se forma la discontinuidad se puede determinar a partir de la condición $\left(\partial x / \partial t_{1}\right)_{t}=0$ para $t_{1}=0$ :

$$
t_{c}=\frac{\xi}{\alpha}
$$

La localización del lugar donde se formará la discontinuidad se obtiene de nuevo de la Ecuación (2.18),

$$
x_{c}=c_{0} t_{c}=\frac{3}{2} \xi^{2} \beta .
$$


La discontinuidad se formará en el extremo más avanzado de la ola, donde la derivada segunda de la Ecuación (2.12) no tiene que ser nula.

Para $\xi>\frac{4}{3}$, la discontinuidad se formará en un instante que vendrá dado por la Ecuación (2.21). Esto sucede en un punto intermedio entre el pistón y el extremo más avanzado de la ola, cuya localización se puede determinar introduciendo las Ecuaciones (2.20) y (2.21) en la Ecuación (2.18):

$$
x_{c}=\beta\left[5 \xi-4-\ln \left(\xi-\frac{1}{3}\right)\right] \text {. }
$$

Puesto que la velocidad del pistón se mantiene constante cuando la superficie libre del metal alcanza el techo de la cámara de inyección, más adelante se discutirá por qué las Ecuaciones (2.21) y (2.27) pueden no ser válidas en algunos casos debido a que la condición expresada por la Ecuación (2.12) no se puede imponer.

\subsubsection{Fracción inicial de llenado mínima}

Como se discutió anteriormente, la ola producida por el movimiento del pistón debería aumentar el nivel del metal fundido lo suficiente para llenar completamente la sección de la cámara de inyección. Una vez que este nivel ha sido alcanzado, el pistón debería moverse a una velocidad constante. En esta sección se buscará el valor mínimo de la fracción inicial de llenado, $f$, necesario para evitar que la ola se rompa antes de que el metal fundido alcance el techo de la cámara $(y=H)$ en la superficie del pistón. Este valor de $f$ se puede determinar imponiendo la condición de que la altura de la ola en la superficie del pistón llegue a ser igual a $H$ en un instante $t_{H}=t_{c}$. La velocidad del pistón en este instante, $u_{H}$, se puede obtener de la Ecuación (2.5), haciendo $u=u_{H}$ y $c=\sqrt{g H}$ :

$$
u_{H}=2\left(\sqrt{g H}-\sqrt{g h_{0}}\right),
$$

y $t_{H}$ se puede determinar sustituyendo $u_{H}=X^{\prime}(t)$ de la Ecuación (2.28) en la Ecuación (2.15):

$$
t_{H}=\frac{1}{\alpha} \ln \left[\frac{2\left(\sqrt{g H}-\sqrt{g h_{0}}\right)}{\alpha \beta}+1\right] .
$$

Igualando esta expresión a las Ecuaciones (2.21) y (2.25), resulta

$$
f_{\min }= \begin{cases}{\left[\frac{3 \xi}{3 \xi+\left(\xi-\frac{1}{3}\right) \mathrm{e}^{4 / 3}-1}\right]^{2},} & \text { si } \xi>\frac{4}{3}, \\ \left(\frac{3 \xi}{3 \xi+\mathrm{e}^{\xi}-1}\right)^{2}, & \text { si } \xi \leq \frac{4}{3} .\end{cases}
$$




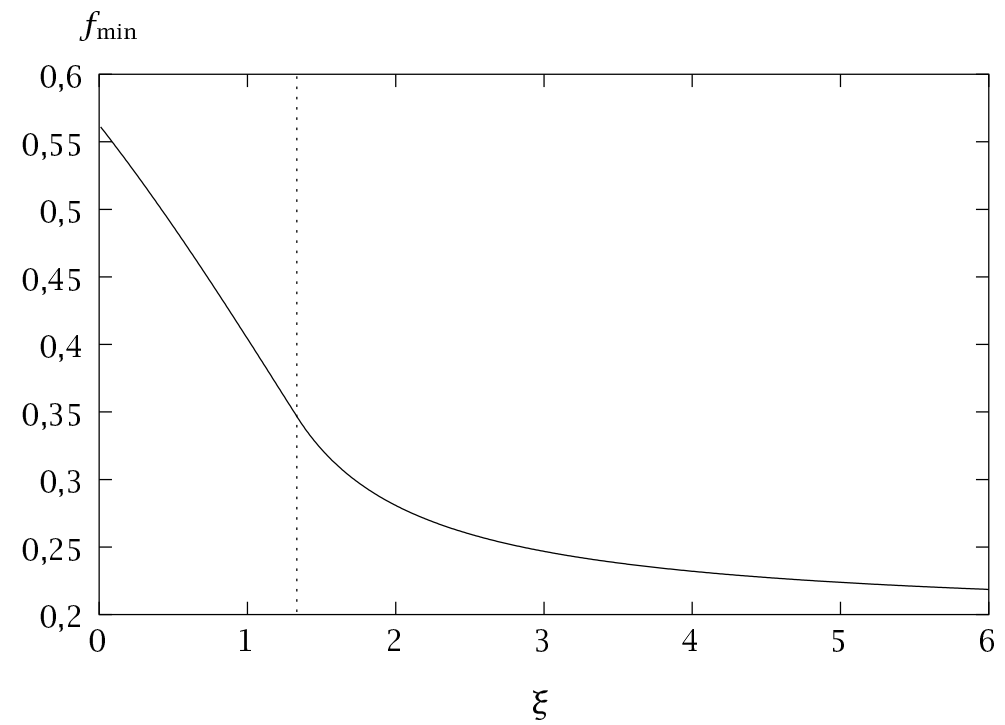

Figura 2.4: Fracción inicial de llenado mínima, $f_{\min }$, en función de $\xi$, para la ley de movimiento de la Ecuación (2.15). 
Esta expresión, representada en la Figura 2.4, proporciona la fracción inicial de llenado mínima en función de $\xi$. Para $f<f_{\min }$, la ola no puede alcanzar el techo de la cámara antes de que se rompa.

Se debe mencionar que la Ecuación (2.28), que proporciona la velocidad crítica del pistón, es idéntica a la obtenida por Tszeng y Chu (1994). En la Tabla 2.1 se muestran otras dos expresiones analíticas propuestas por otros autores.

\begin{tabular}{|l|l|}
\hline Garber (1982) & $u_{H}=\left[2 g H(1-f)^{2} /(1+f)\right]^{1 / 2}$ \\
\hline Karni (1991) & $u_{H}=\left[g H f^{-1}(1+f)(1-f)^{2} / 2\right]^{1 / 2}$ \\
\hline Tszeng y Chu (1994) & $u_{H}=2 \sqrt{g H}\left(1-f^{1 / 2}\right)$ \\
\hline
\end{tabular}

Tabla 2.1: Expresiones analíticas de la velocidad crítica.

\subsubsection{Parámetros óptimos de aceleración}

Como se ha mencionado, el movimiento del pistón en la cámara debería evitar la rotura de la ola durante el proceso de llenado y minimizar los efectos de atrapamiento de aire debidos al impacto del metal fundido en la pared final de la cámara de inyección. Además, con objeto de reducir el tiempo de la fase lenta para conseguir altas velocidades de producción y evitar la solidificación del metal durante el proceso de llenado, la ola de metal fundido debería empezar a romperse justo en $x=x_{c}=L$.

Si se impone como condición el que la discontinuidad se forme justo en la pared final de la cámara, todas la condiciones de trabajo en las que la discontinuidad aparezca en cualquier sección localizada entre el pistón y la parte delantera de la ola producirá la reflexión de la ola antes de que empiece a romperse en $x=L^{*}$. Como se discutió anteriormente, tales condiciones corresponden a $\xi>\frac{4}{3}$. Como la velocidad del pistón se mantiene constante para $t>t_{H}$, el instante en el que se forma la discontinuidad vendrá dado por las Ecuaciones (2.20) y (2.21) siempre que $t_{1} \leq t_{H}$. Sustituyendo $t_{1}$ obtenido de las Ecuaciones (2.20) y (2.21),

$$
t_{1}=\frac{1}{\alpha} \ln \left(\xi-\frac{1}{3}\right),
$$

\footnotetext{
* En lo que sigue no se considerará la presencia de la pared final de la cámara, de forma que no se tendrá en cuenta la reflexión de la ola en dicha pared. Estos efectos sí se tendrán en cuenta en el Capítulo 3.
} 
y $t_{H}$ de la Ecuación (2.29) en la condición $t_{1} \leq t_{H}$, se obtiene

$$
f \leq f^{*}=\left[\frac{9 \xi}{4(3 \xi-1)}\right]^{2} .
$$

Para $\xi \rightarrow \infty$, el valor máximo de $f$ dado por la Ecuación (2.32), $f^{*}$, tiende a $\frac{9}{16}$; para $\xi \rightarrow \frac{4}{3}, f^{*} \rightarrow 1$. Haciendo $x_{c}=L$ en la Ecuación (2.27), se consigue

$$
\frac{L}{\beta}=5 \xi-4-\ln \left(\xi-\frac{1}{3}\right),
$$

lo que proporciona, para un $L$ y $h_{0}$ dados, la relación que debe existir entre $\alpha$ y $\beta$ para que la ola empiece a romperse justo en la pared final de la cámara cuando $\xi>\frac{4}{3}$ y cuando se satisfaga la condición de la Ecuación (2.32).

Para $\xi>\frac{4}{3}$ y $f>f^{*}$, la condición expresada por la Ecuación (2.12) se debe reemplazar por $t_{1}=t_{H}$, donde $t_{H}$ viene dado por la Ecuación (2.29). La condición de que la discontinuidad aparezca en la pared final de la cámara de inyección se puede imponer en este caso haciendo $x=L$ y $t=t_{c}$ en la Ecuación (2.8), y sustituyendo en la expresión resultante la expresión para $t_{c}-t_{1}$ que se obtiene de la Ecuación (2.19) y $t_{1}=t_{H}$ de la Ecuación (2.29). Reagrupando términos, se obtiene finalmente

$$
\frac{L}{\beta}=\frac{\frac{3}{2} \xi^{2} f^{-1 / 2}\left(3 f^{-1 / 2}-2\right)}{3 \xi\left(f^{-1 / 2}-1\right)+1}+3 \xi\left(f^{-1 / 2}-1\right)-\ln \left[3 \xi\left(f^{-1 / 2}-1\right)+1\right],
$$

que es la relación equivalente a la de la Ecuación (2.33) para casos con $f>f^{*}$. En el caso límite de $f=f^{*}$, la Ecuación (2.34) se reduce a la Ecuación (2.33). Se quiere hacer notar de nuevo que las Ecuaciones (2.33) y (2.34) se han obtenido sin tener en cuenta que el extremo de la ola empezará a reflejarse en la pared final de la cámara de inyección antes de que la discontinuidad pueda formarse en $x=L$. En el siguiente capítulo se extenderá el modelo desarrollado considerando los efectos de reflexión de la ola de metal fundido en la pared final de la cámara de inyección, y se analizarán los casos en los que dichos efectos puedan ser importantes.

Para casos con $\xi \leq \frac{4}{3}$, la expresión equivalente a las Ecuaciones (2.33) y (2.34) se obtiene haciendo $x_{c}=L$ en la Ecuación (2.26):

$$
\frac{L}{\beta}=\frac{3}{2} \xi^{2} \text {. }
$$

En este caso no hay reflexión de la ola antes de que la discontinuidad se forme en $x=L$.

A continuación se va a buscar un valor óptimo para $\xi \leq \frac{4}{3}$ que, satisfaciendo la Ecuación (2.35), minimice el volumen de aire atrapado. Para 
este propósito, se supondrá que el volumen de aire atrapado es proporcional al volumen de aire dentro de la cámara de inyección en el instante $t_{c}$. Como cabía esperar, se ha encontrado que en estos casos, y para un valor de $L$ dado, este volumen decrece con la distancia $x_{T}$ recorrida por la parte superior del frente de la ola en el instante $t=t_{c}$. Esta distancia, adimensionalizada con el parámetro $\beta$, se puede expresar en función de la fracción inicial de llenado y $\xi$. Sustituyendo $X\left(t_{1}\right)$ de la Ecuación (2.17), $X^{\prime}\left(t_{1}\right)$ de la Ecuación (2.9) y $t_{1}$ despejado de la Ecuación (2.15) (haciendo en ésta $t=t_{1}$ ) en la Ecuación (2.8), se obtiene

$$
x=\beta\left[\frac{u}{\alpha \beta}-\ln \left(\frac{u}{\alpha \beta}+1\right)\right]+\left(\frac{3}{2} u+c_{0}\right)\left[t-\frac{1}{\alpha} \ln \left(\frac{u}{\alpha \beta}+1\right)\right],
$$

y expresando $u$ en función de $c$ a partir de la Ecuación (2.5) y sustituyendo $t$ por el valor de $t_{c}$ dado por la Ecuación (2.25) en la Ecuación (2.36), y haciendo $c=\sqrt{g H}$, resulta

$$
\begin{aligned}
\frac{x_{T}}{\beta}=3 \xi^{2}\left(\frac{3}{2} f^{-1 / 2}-1\right)+3 & \xi\left(f^{-1 / 2}-1\right) \\
& -\left[3 \xi\left(\frac{3}{2} f^{-1 / 2}-1\right)+1\right] \ln \left[3 \xi\left(f^{-1 / 2}-1\right)+1\right] .
\end{aligned}
$$

Introduciendo la condición expresada por la Ecuación (2.35) en la Ecuación (2.37), se obtiene finalmente

$$
\begin{aligned}
\frac{x_{T}}{L}=3 f^{-1 / 2}-2+ & 2\left(f^{-1 / 2}-1\right) / \xi \\
& -\left[\xi\left(3 f^{-1 / 2}-2\right)+\frac{2}{3}\right] \ln \left[3 \xi\left(f^{-1 / 2}-1\right)+1\right] / \xi^{2},
\end{aligned}
$$

que proporciona $x_{T} / L$ en función de $\xi$ para un valor de $f$ dado. Como $x_{T} / L$ aumenta con $\xi$ para un valor de $f$ dado, se puede concluir que $\xi=\frac{4}{3}$ es, en el rango considerado de $\xi \leq \frac{4}{3}$, el valor óptimo de $\xi$ que haría que la ola empiece a romperse en $x=L$ cuando la Ecuación (2.35) se satisface. Además, es importante observar que $t_{c}=L / c_{0}$ es constante para $\xi \leq \frac{4}{3}$, y un valor de $f$ y unas características geométricas de la cámara de inyección dadas, por lo que, como el aire residual en la cámara de inyección es mínimo para $\xi=\frac{4}{3}$, el tiempo de la fase lenta, que es la suma de $t_{c}$ y el tiempo necesario para evacuar el aire residual en $t_{c}$, también será mínimo para $\xi=\frac{4}{3}$.

En la Figura 2.5 se representan los perfiles de ola en $t=t_{c}$ que satisfacen la condición $x_{c}=L$, para diferentes valores de la fracción inicial de llenado y $\xi$. Según lo anterior, para valores de $\xi>\frac{4}{3}$ la ola se romperá en algún punto entre el extremo más avanzado y la parte superior de la ola. Para fracciones de llenado inferiores a 9/16, existe un valor de $\xi$ por debajo del cual la ola no alcanzará el techo de la cámara de inyección antes 

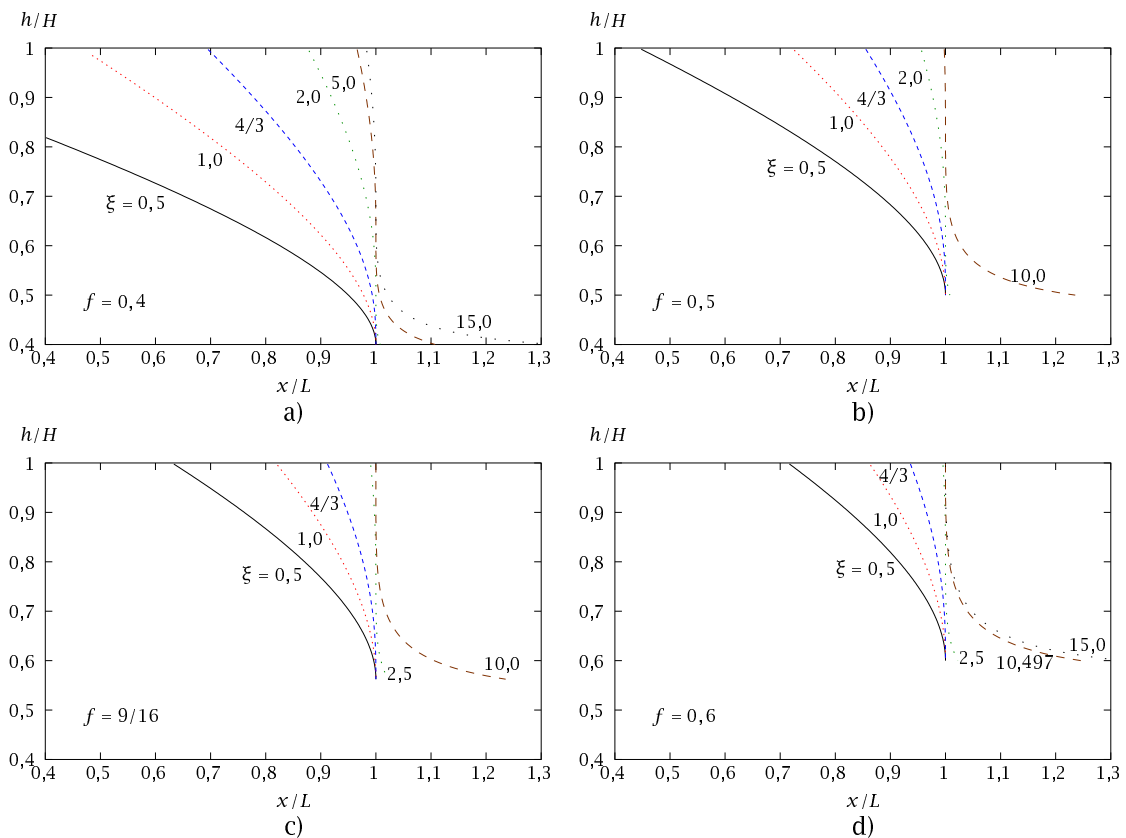

Figura 2.5: Perfiles de la ola en $t=t_{c}$, satisfaciendo la condición $x_{c}=L$, para la ley de movimiento de la Ecuación (2.15) y diferentes valores de la fracción inicial de llenado y $\xi$. 
de romperse. Por ejemplo, de la Ecuación (2.30) representada en la Figura 2.4, los valores límite de $\xi$ son 1,025 y 0,420 para $f=0,4$ y $f=0,5$, respectivamente (Figuras 2.5a y 2.5b). En la Figura 2.5a se puede ver que para los valores representados de $\xi=0,5$ y $\xi=1$, el metal fundido no alcanza el techo de la cámara de inyección antes de que la ola empiece a romperse. Por otro lado, para fracciones de llenado superiores a 9/16 existe un valor límite de $\xi$, por encima del cual la discontinuidad se formará en la parte más alta del frente de la ola. Por ejemplo, para $f=0,6$ se obtiene de la Ecuación (2.32) un valor límite de $\xi=10,497$ (Figura 2.5 d). Obsérvese que para valores muy grandes de $\xi$ la ola empezará a reflejarse bastante tiempo antes de $t_{c}$. Se puede ver asimismo que para valores de $\xi$ por encima de $4 / 3$, el volumen de aire que permanece en la cámara en el instante $t_{c}$ llega a ser mucho menor que para $\xi=4 / 3$, por lo que, incluso aunque el frente delantero de la ola empiece a reflejarse contra la pared final de la cámara de inyección en un instante anterior a $t_{c}$ cuando $\xi>4 / 3$, el valor óptimo de $\xi$ será probablemente algo mayor que $4 / 3$.

Es interesante comparar lo tiempos de llenado $t_{f}$ correspondientes a cada uno de los perfiles de ola representados en la Figura 2.5. El tiempo de llenado se puede expresar como la suma de $t_{H}$ y el tiempo restante hasta que la cámara esté completamente llena una vez que el metal fundido alcanza el techo de la cámara de inyección:

$$
t_{f}=t_{H}+\frac{L(1-f)-X\left(t_{H}\right)}{u_{H}} .
$$

Sustituyendo las Ecuaciones (2.17), (2.28) y (2.29) en la Ecuación (2.39) se obtiene

$$
\begin{aligned}
\frac{t_{f}}{L / c_{0}}=\frac{1}{L / \beta}\left\{\left[\frac{3}{2} \xi+\frac{1}{2\left(f^{-1 / 2}-1\right)}\right] \ln \right. & {\left[3 \xi\left(f^{-1 / 2}-1\right)+1\right] } \\
- & \left.\frac{3}{2} \xi\right\}+\frac{(1-f)}{2\left(f^{-1 / 2}-1\right)} .
\end{aligned}
$$

Sustituyendo $L / \beta$ de las Ecuaciones (2.33), (2.34) y (2.35), para sus respectivos rangos de validez, en la Ecuación (2.40), se obtiene el tiempo de llenado, adimensionalizado con $L / \mathcal{c}_{0}$, en función de $\xi$ y $f$ para los perfiles de ola mostrados en la Figura 2.5. Es importante remarcar que $L / c_{0}$ es el tiempo que tarda el extremo más avanzado del frente de la ola en alcanzar la pared final de la cámara. El tiempo de llenado adimensionalizado se representa en la Figura 2.6 en función de $\xi$ para diferentes valores de $f$ y valores de $\xi$ mayores que el límite dado por la Ecuación (2.30) para cada valor de $f$. En esta figura se puede ver que, para un valor de $f$ dado, el tiempo mínimo de llenado se obtiene para valores de $\xi$ alrededor de 2, tendiendo a $\xi=4 / 3$ en el límite $f \rightarrow 1$. Claramente, $t_{f}$ se debe mantener tan pequeño como sea posible para reducir el tiempo total de inyección. 


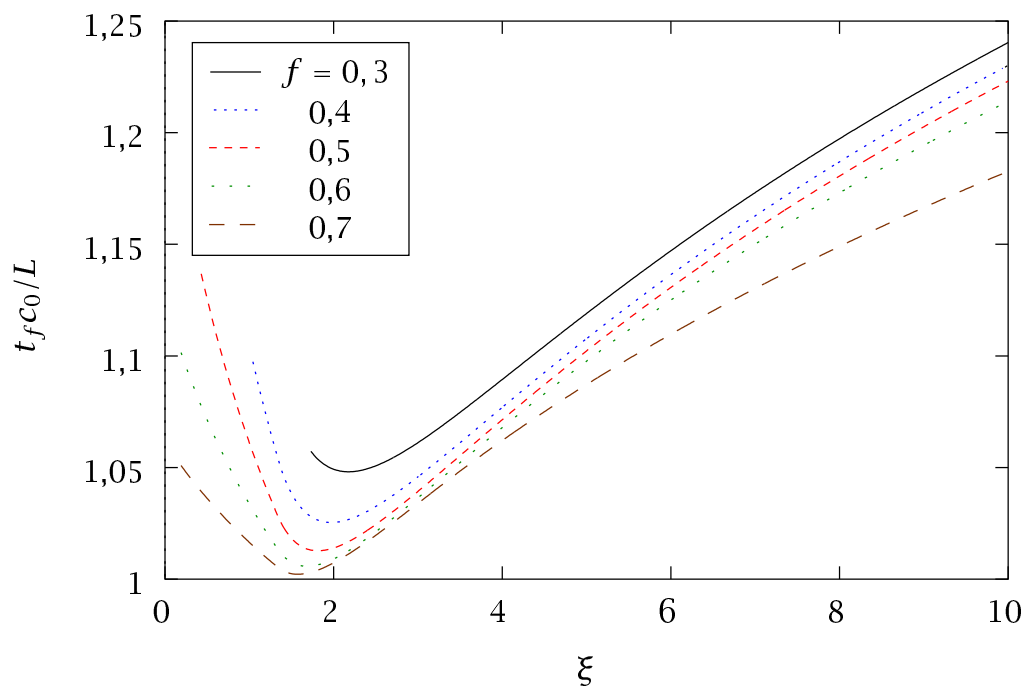

Figura 2.6: Tiempo adimensional de llenado en función de $\xi$ para diferentes valores de $f$ con la ley de movimiento de la Ecuación (2.15). 
También sería interesante hacer una estimación de la cantidad de aire atrapado, y para ello se va a suponer que esta cantidad está relacionada con la masa de aire que se encuentra en la cámara de inyección en el instante $t=t_{c}$. Como el problema se ha tratado bidimensionalmente, la masa de aire en el interior de la cámara en $t=t_{c}$ se tomará igual a la diferencia entre el área ocupada por el aire en la cámara y el área del metal fundido en $x>L$, fuera de los límites de la cámara (véanse los perfiles de las olas en la Figura 2.5), que puede ser expresada como

$$
A=L H\left[1-f-\frac{X\left(t_{c}\right)}{L}\right] .
$$

La Figura 2.7 muestra, para la ley de aceleración exponencial, los resultados obtenidos del área adimensional $A /(H L)$ en función de $\xi$ para diferentes fracciones iniciales de llenado. Los parámetros de aceleración se han elegido para que la ola empiece a romperse exactamente (el perfil se haga vertical) en $x_{c}=L$. Es importante destacar que el área adimensional $A /(H L)$ disminuye más rápidamente conforme $\xi$ aumenta para altas fracciones iniciales de llenado, y llega a ser baja, en términos relativos, para valores de $\xi$ próximos al valor correspondiente al tiempo de llenado mínimo de la Figura 2.6.

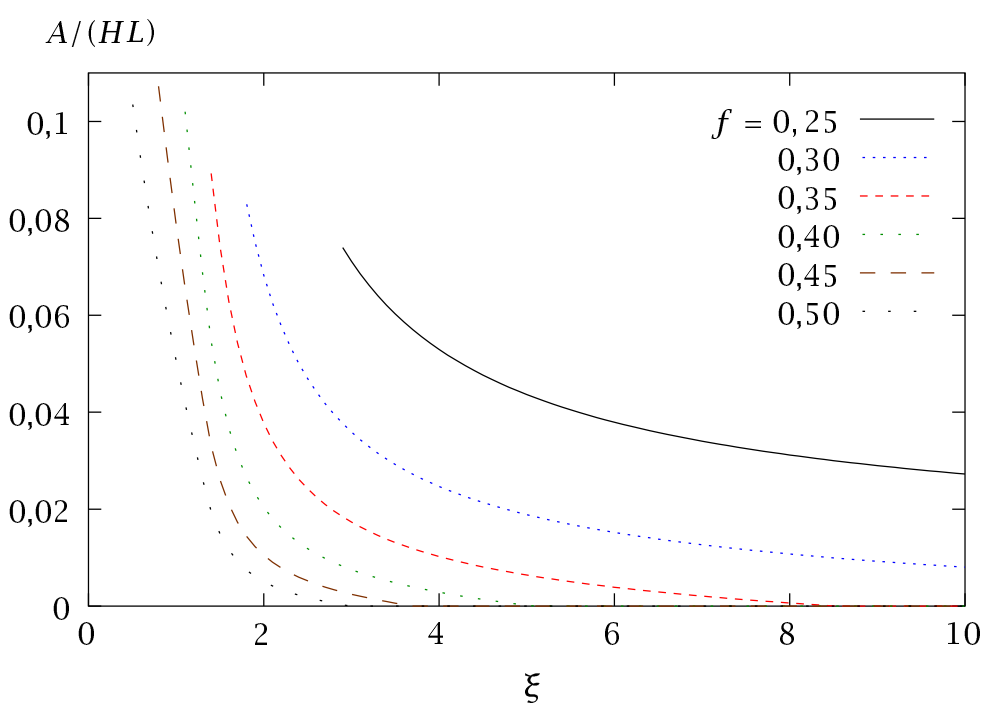

Figura 2.7: Área adimensional ocupada por el aire en la cámara de inyección en $t=t_{c}$, en función de $\xi$, para una ley de aceleración exponencial con la que se obtiene $x_{c}=L$. 


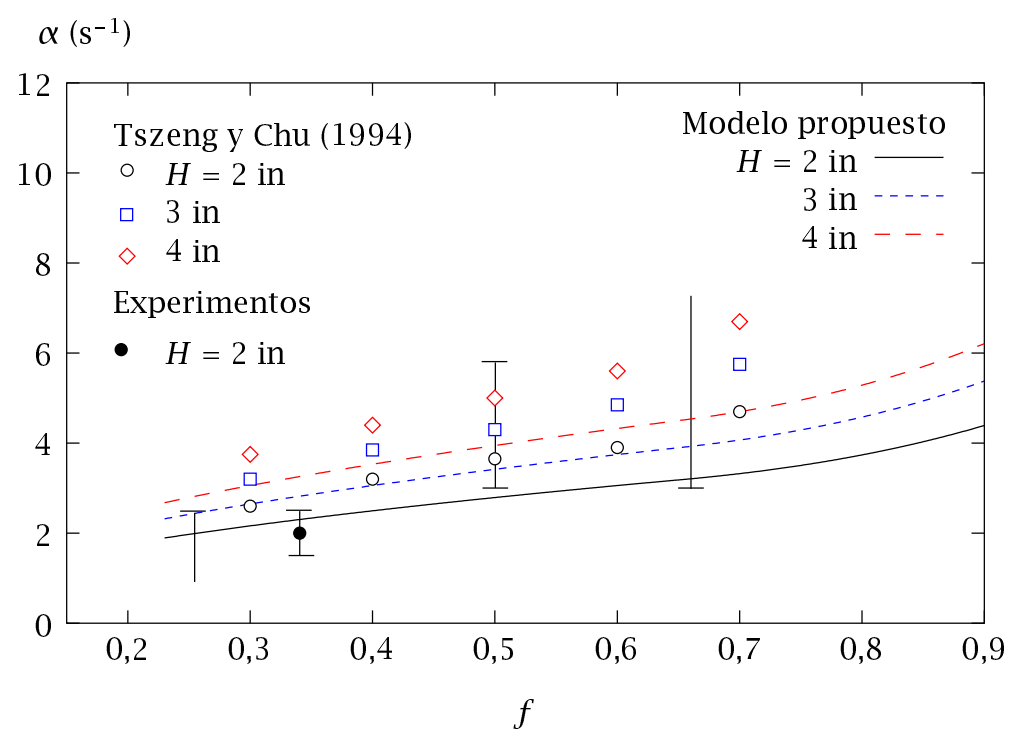

Figura 2.8: Comparación de los valores óptimos de $\alpha$ predichos en este trabajo con el modelo analítico sin reflexión y los obtenidos por Tszeng y Chu (1994) para una carrera del pistón $L=45,72 \mathrm{~cm}$ (18 in), con las mediciones experimentales de Duran et al. (1991).

Teniendo todo lo anterior en cuenta, se puede concluir que el valor óptimo de $\xi$ dependerá de $f$ y será en todo caso mayor que $4 / 3$. La determinación exacta de la influencia de $\xi$ en los efectos del aire atrapado, para las condiciones de operación representadas en la Figura 2.5, para las que $x_{c}=L$, dependerá del análisis detallado del proceso de llenado de la cámara de inyección después de que la parte delantera de la ola alcance la pared final de la cámara, que se llevará a cabo en los dos capítulos siguientes.

\subsubsection{Comparación con otros resultados analíticos y expe- rimentales}

En la Figura 2.8 se compara el valor del parámetro de aceleración óptimo $\alpha$ predicho en este trabajo y el que obtuvo Tszeng y Chu (1994) para casos con carrera del pistón $L=45,72 \mathrm{~cm}$ (18 in), diámetros de la cámara de inyección $H=5,08,7,62$ y 10,16 cm (2, 3 y 4 in), y porcentajes iniciales de llenado entre un $20 \%$ y un $70 \%$. El parámetro $\beta$ se ha tomado igual a 
2,54 cm (1 in) para reproducir la ley del movimiento del pistón empleada por Tszeng y Chu (1994). Estos autores obtuvieron sus resultados analizando los perfiles del frente de la ola de metal fundido para diferentes valores de $\alpha$ en el momento en el que la parte superior del frente de la ola había recorrido una distancia $L=45,72 \mathrm{~cm}$ (18 in) desde $x=0$. La idea fue determinar la aceleración más alta posible que no hiciese que la inclinación del frente de la ola fuese vertical. Se puede ver que el presente modelo predice valores de la aceleración óptima más bajos que los obtenidos por Tszeng y Chu (1994), aunque la concordancia global entre ambos es cualitativamente buena.

Se puede comprobar que $\xi$ es mayor que $\frac{4}{3}$ para todas las condiciones representadas en la Figura 2.8, por lo que los valores óptimos de $\alpha$ se han determinado en cualquier caso de la Ecuación (2.33) cuando $f \leq f^{*}$, o de la Ecuación (2.34) cuando $f>f^{*}$. Se puede observar que la Ecuación (2.33) proporciona un valor de $\xi=4,695$, para el que la Ecuación (2.32) da $f^{*}=0,652$. Para este valor de $\xi=4,695$, la Ecuación (2.30) proporciona una fracción mínima de llenado de 0,226; para fracciones más bajas, la ola empezará a romperse antes de que el metal fundido alcance el techo de la cámara de inyección, lo que está de acuerdo con los resultados de Tszeng y Chu (1994) para una fracción de llenado de 0,2. Se espera que aceleraciones del pistón inferiores a las predichas por las Ecuaciones (2.33) y (2.34) podrían producir no sólo mayores tiempos de llenado, sino también un aumento del aire atrapado debido a los efectos de la reflexión de la ola. En los capítulos siguientes se estudiará, considerando los efectos de reflexión de la ola en la pared final de la cámara, la influencia de los parámetros de aceleración sobre la cantidad de aire atrapado.

Duran et al. (1991) realizaron una serie de experimentos con agua para una cámara de inyección de sección circular de $L=45,72 \mathrm{~cm}$ (18 in) y diámetro de $5,08 \mathrm{~cm}$ (2 in), y midieron el volumen de aire atrapado durante el proceso de llenado para diferentes aceleraciones del pistón. Sus experimentos se llevaron a cabo con el mismo tipo de movimiento del pistón que el usado por Tszeng y Chu (1994), excepto en que Duran et al. (1991) usó una velocidad crítica $u_{H}$ mayor que la obtenida por la Ecuación (2.28). Los resultados de los experimentos realizados por Duran et al. (1991) se muestran en la Figura 2.9. Obsérvese que la fracción inicial de llenado representada en esta figura, $f^{\prime}$, es la fracción volumétrica correspondiente a la sección circular. Para $f^{\prime}=0,3$, encontraron el valor mínimo de aire atrapado para un parámetro de aceleración $\alpha=2 \mathrm{~s}^{-1}$; de la Figura 2.9, se deduce que el óptimo debe situarse entre 1,5 y 2,5 s-1. Para $f^{\prime}=0,2,0,5$ y 0,7 , las mediciones experimentales no permiten identificar un volumen mínimo de aire atrapado en el rango de aceleraciones consideradas. Sin embargo, se puede deducir de estas mediciones que este mínimo se encontrará probablemente para valores de $\alpha$ inferiores 


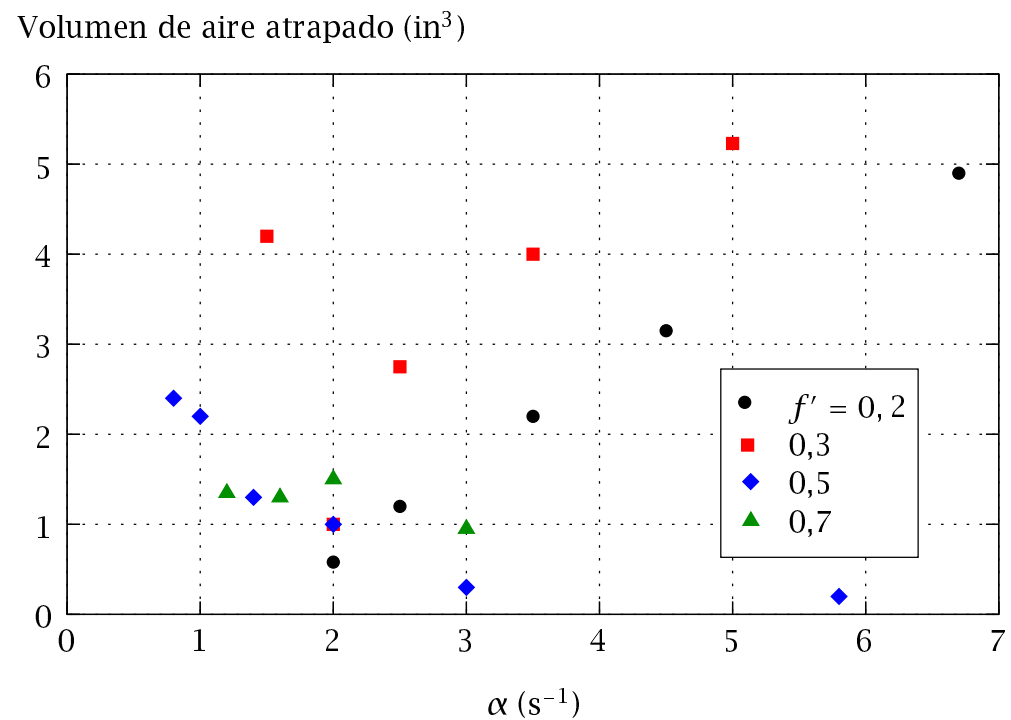

Figura 2.9: Medidas experimentales del volumen de aire atrapado de Duran et al. (1991). 
a 2,5 $\mathrm{s}^{-1}$ cuando $f^{\prime}=0,2$ (el mínimo medido en el rango de las aceleraciones experimentales corresponde a $\alpha=2 \mathrm{~s}^{-1}$ ), y por encima de 3 $\mathrm{s}^{-1}$ cuando $f^{\prime}=0,7$. Para $f^{\prime}=0,5$, la cantidad mínima de aire atrapado probablemente corresponderá a un valor de $\alpha$ entre 3 y 5,8 s $\mathrm{s}^{-1}$. Se puede ver, por lo tanto, que la comparación de estos resultados experimentales con los del modelo propuesto y los de Tszeng y Chu (1994) es más interesante cuando $f^{\prime}=0,3$, y menos concluyente cuando $f^{\prime}=0,2$, 0,5 y 0,7 , por lo que sería necesario conocer resultados experimentales para más amplios rangos de aceleración. La Figura 2.8 muestra el valor de $\alpha$ correspondiente al mínimo volumen de aire atrapado medido para $f^{\prime}=0,3$, que corresponde a $f=0,340$ en el plano de simetría; dentro de las limitaciones e incertidumbres que se acaban de mencionar, en este caso hay un buen grado de concordancia con el resultado obtenido en nuestro estudio. Para $f^{\prime}=0,2,0,5$ y 0,7 , que corresponden respectivamente a $f=0,254,0,5$ y 0,660 en el plano de simetría, los rangos de los posibles valores del óptimo $\alpha$, según los experimentos, se indican en la Figura 2.8 con líneas verticales. También la concordancia entre las predicciones y experimentos para $f^{\prime}=0,2$ es buena. Cuando $f^{\prime}=0,5$ ó 0,7 , las mediciones experimentales parecen indicar que los valores del parámetro de aceleración óptima $\alpha$ están probablemente más cerca de los predichos por Tszeng y Chu (1994).

Es importante remarcar que cuando el valor de $\beta$ es fijo, como se ha tomado en esta sección, no es posible seleccionar independientemente un valor óptimo de $\xi$. Sin embargo, habitualmente deberá ser posible variar el parámetro $\beta$, por lo que la flexibilidad para elegir las variables del proceso será mayor.

\subsection{Movimiento del pistón con aceleración poten- cial}

A continuación se considerará el caso de movimiento del pistón desde el reposo con una velocidad

$$
X^{\prime}(t)=\gamma t^{n}
$$

siendo $\gamma$ y $n$ constantes positivas, lo que proporciona una aceleración

$$
X^{\prime \prime}(t)=n \gamma t^{n-1} .
$$

Este tipo de ley de movimiento es típico en máquinas de fundición en las que la velocidad del pistón se especifica en función del tiempo. Integrando la Ecuación (2.42) y suponiendo que la localización inicial de la superficie del pistón está en $x=0$, se obtiene

$$
X(t)=\frac{\gamma}{n+1} t^{n+1} .
$$


Landau y Lifshitz (1959) presentaron un análisis similar al que se hace aquí para el caso del flujo unidimensional de un gas en un tubo cilíndrico semi-infinito limitado por un pistón. Cuando $n<1$, se puede demostrar fácilmente que, para un instante dado, $h(x)$ es una función múltiple para cualquier $t>0$, por lo que se formará una discontinuidad en la superficie del pistón en $t=0$. (Se puede ver que, para $n<1$, el pistón se mueve con aceleración infinita en $t=0$.)

Sustituyendo las Ecuaciones (2.42) y (2.44) en la Ecuación (2.8), y aplicando las condiciones expresadas por las Ecuaciones (2.11) y (2.12), se obtiene

$$
t_{c}=\left(\frac{2 c_{0}}{3 \gamma}\right)^{1 / n}\left[\frac{3 n+1}{3(n-1)}\right]^{(n-1) / n}
$$

y

$$
t_{1}=\left(\frac{2 c_{0}}{\gamma} \frac{n-1}{3 n+1}\right)^{1 / n} .
$$

El valor mínimo de la fracción inicial de llenado se determina, como en la sección anterior, imponiendo la condición de que la altura de la ola en la superficie del pistón llegue a ser igual a $H$ en un instante $t_{H}=t_{c}$. Sustituyendo $u_{H}=X^{\prime}(t)$ de la Ecuación (2.28) en la Ecuación (2.42), se obtiene

$$
t_{H}=\left[\frac{2 c_{0}}{\gamma}\left(f^{-1 / 2}-1\right)\right]^{1 / n},
$$

e igualando esta expresión a la Ecuación (2.45), resulta

$$
f_{\min }=\frac{1}{\left[\frac{1}{3^{n}}\left(\frac{3 n+1}{n-1}\right)^{n-1}+1\right]^{2}}
$$

Esta expresión, representada en la Figura 2.10, se puede comparar con la correspondiente a la ley exponencial, dada por la Ecuación (2.30).

Para $n>1$ la discontinuidad se formará en algún punto intermedio entre la parte delantera de la ola y la superficie del pistón, por lo que no es posible que la ola empiece a romperse en la pared final de la cámara antes de que su parte delantera se refleje contra ella. Al igual que en la sección anterior, como la velocidad del pistón se mantiene constante para $t>t_{H}$, el instante en el que se forma la discontinuidad estará determinado por la Ecuación (2.45) siempre que $t_{1} \leq t_{H}$. Sustituyendo $t_{1}$ de la Ecuación (2.46) y $t_{H}$ de la Ecuación (2.47) en la desigualdad $t_{1} \leq t_{H}$, se obtiene

$$
f \leq f^{*}=\left(\frac{3 n+1}{4 n}\right)^{2} .
$$

El valor máximo de $f$ dado por la Ecuación (2.49), $f^{*}$, tiende a $\frac{9}{16}$ para $n \rightarrow \infty$ y a 1 para $n \rightarrow 1$. Haciendo $x=L$ y $t=t_{c}$, e introduciendo $t_{1}$ de 


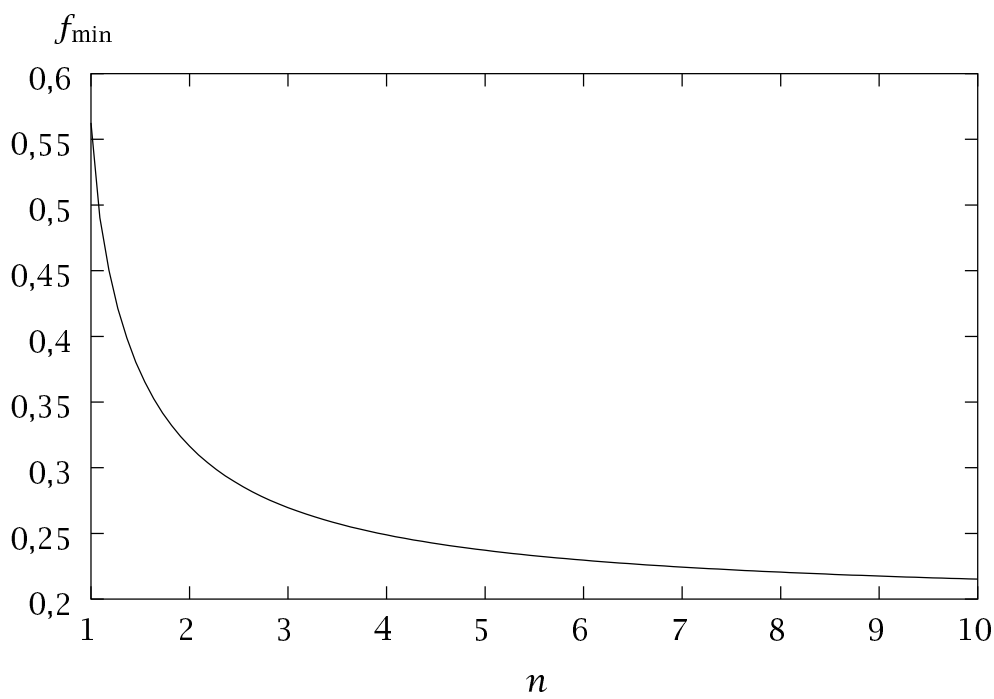

Figura 2.10: Fracción inicial de llenado mínima, $f_{\min }$, en función de $n$, para la ley de movimiento de la Ecuación (2.42).

la Ecuación (2.46) y $t_{c}$ de la Ecuación (2.45) en la Ecuación (2.8), resulta

$$
\frac{L \gamma^{1 / n}}{c_{0}^{(n+1) / n}}=\frac{5 n^{2}+\frac{1}{3}(2 n-1)}{(n+1)(n-1)^{2}}\left(2 \frac{n-1}{3 n+1}\right)^{(n+1) / n},
$$

lo que proporciona, para un valor dado de $L$ y $h_{0}$, la relación requerida entre $n$ y $\gamma$ para hacer que la ola empiece a romperse en la pared final de la cámara cuando $n>1$ y se satisface la condición de la Ecuación (2.49). La relación equivalente a la Ecuación (2.34) correspondiente a $f>f^{*}$ se puede obtener, también en este caso, reemplazando la condición de la Ecuación (2.12) por $t_{1}=t_{H}$,

$$
\begin{aligned}
\frac{L \gamma^{1 / n}}{c_{0}^{(n+1) / n}}=\frac{1}{n+1} & {\left[2\left(f^{-1 / 2}-1\right)\right]^{(n+1) / n} } \\
+ & \frac{2 f^{-1 / 2}\left(3 f^{-1 / 2}-2\right)}{3 n}\left[2\left(f^{-1 / 2}-1\right)\right]^{(1-n) / n} .
\end{aligned}
$$

Para $n=1$, de la Ecuación (2.46) se obtiene $t_{1}=0$, por lo que la discontinuidad se formará en el extremo del frente de la ola en el instante

$$
t_{c}=\frac{2 c_{0}}{3 \gamma} \text {. }
$$


El lugar en el que se forma la discontinuidad se puede obtener sustituyendo $t_{c}$ de la Ecuación (2.52) en la Ecuación (2.8) con $t_{1}=0$, y es obviamente

$$
x_{c}=c_{0} t_{c}=\frac{2 c_{0}^{2}}{3 \gamma} .
$$

Introduciendo la condición $x_{c}=L$, a partir de la Ecuación (2.53) se consigue la relación equivalente a las de las Ecuaciones (2.50) y (2.51):

$$
\frac{L \gamma}{c_{0}^{2}}=\frac{2}{3}
$$

lo que proporciona la aceleración requerida que hace que la ola empiece a romperse en la pared final de la cámara.

El tiempo de llenado se puede obtener sustituyendo las Ecuaciones (2.44), (2.28) y (2.47) en la Ecuación (2.39):

$$
\frac{t_{f}}{L / c_{0}}=\frac{c_{0}^{(n+1) / n}}{L \gamma^{1 / n}} \frac{n}{n+1}\left[2\left(f^{-1 / 2}-1\right)\right]^{1 / n}+\frac{1-f}{2\left(f^{-1 / 2}-1\right)} .
$$

Para condiciones de operación que satisfacen $x_{c}=L$, el tiempo de llenado se puede obtener sustituyendo $L \gamma^{1 / n} / c_{0}^{(n+1) / n}$ de las Ecuaciones (2.50), (2.51) y (2.54), para sus respectivos rangos de validez, en la Ecuación (2.55). El tiempo de llenado, adimensionalizado con $L / c_{0}$, se representa en la Figura 2.11 en función de $n$ para diferentes valores de $f$, con valores de $n$ mayores que el límite dado por la Ecuación (2.48). Se puede observar que el mínimo tiempo de llenado se obtiene para un valor de $n$ que aumenta conforme $f$ disminuye.

Comparando las Figuras 2.6 y 2.11 podemos concluir que la ley de aceleración exponencial requiere tiempos de llenado más pequeños por lo que es normalmente preferible a la ley de aceleración potencial.

Se puede demostrar fácilmente que para $n=1$ la expresión equivalente a la Ecuación (2.38) es

$$
\frac{x_{T}}{L}=3\left[\left(f^{-1 / 2}-1\right)^{2}-\left(3 f^{-1 / 2}-2\right)\left(f^{-1 / 2}-\frac{4}{3}\right)\right] .
$$

El volumen de aire en la cámara cuando la ola empieza a romperse en $x=L$, en los casos cuando $\xi=\frac{4}{3}$ y $n=1$ (para las leyes de movimiento de las Ecuaciones (2.15) y (2.42), respectivamente) disminuye conforme $x_{T} / L$ aumenta. Ya se mencionó que este volumen de aire podría, de alguna forma, relacionarse con el volumen final de aire atrapado en el metal fundido. La Figura 2.12 muestra $x_{T} / L$ en función de $f$ para los dos casos anteriores. Se puede observar que la ley de movimiento exponencial proporciona valores más grandes de $x_{T}$ y de este modo menores volúmenes de aire atrapado en la cámara en $t=t_{c}$. 


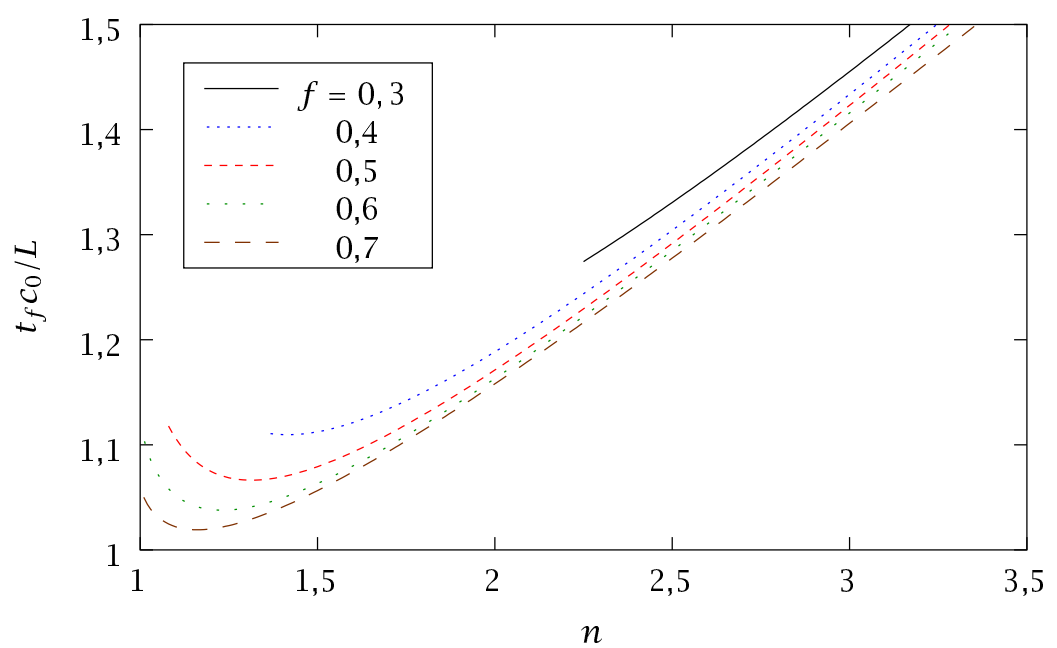

Figura 2.11: Tiempo de llenado adimensional en función de $n$ para diferentes valores de $f$ y la ley de movimiento de la Ecuación (2.42), para condiciones de operación que satisfacen $x_{c}=L$. 


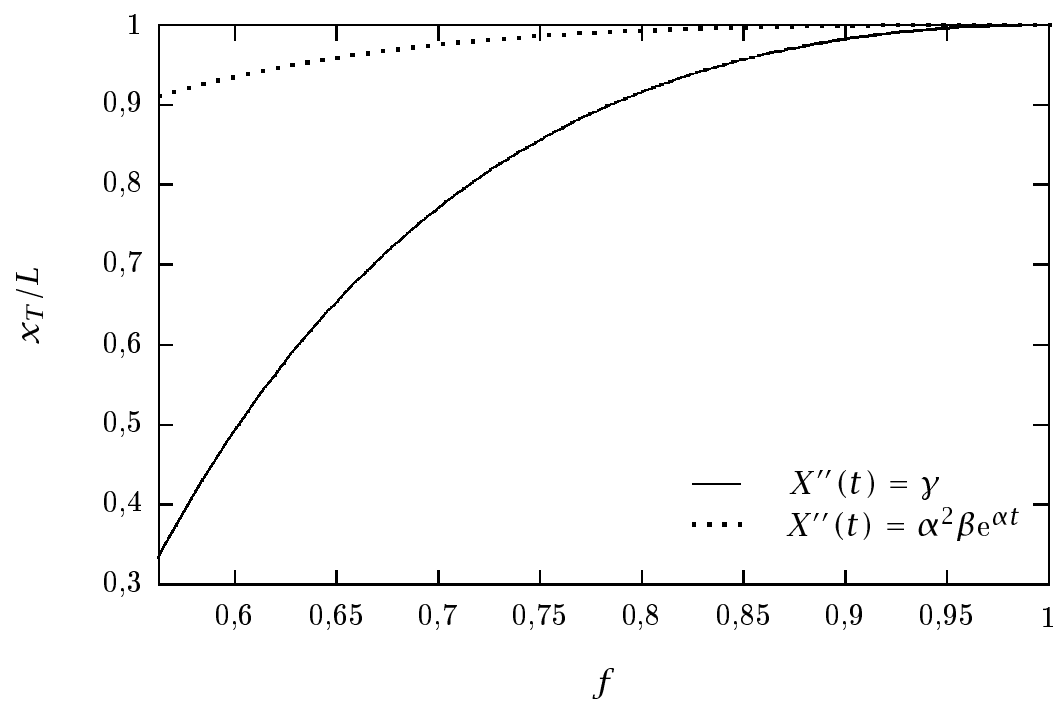

Figura 2.12: Distancia recorrida por la parte superior del frente de ola en $t_{c}$, adimensionalizada con $L$, en función de la fracción inicial de llenado para $x_{c}=L$ y las leyes de movimiento de las Ecuaciones (2.15) y (2.42), con $\xi=\frac{4}{3}$ y $n=1$, respectivamente. 
Para otros valores de $n$, y de igual forma que para la ley de aceleración exponencial, se puede estimar la cantidad de aire atrapado considerando que ésta es proporcional a la masa de aire que hay en la cámara de inyección en el instante en el que se forma una discontinuidad en la superficie libre del metal fundido. En la Figura 2.13 se representa el área de la Ecuación (2.41) ocupada por el aire en la cámara de inyección en el instante $t=t_{c}$, en función de $n$, para una ley de aceleración potencial que satisface la condición $x_{c}=L$. Al igual que ocurre con la ley de acele-

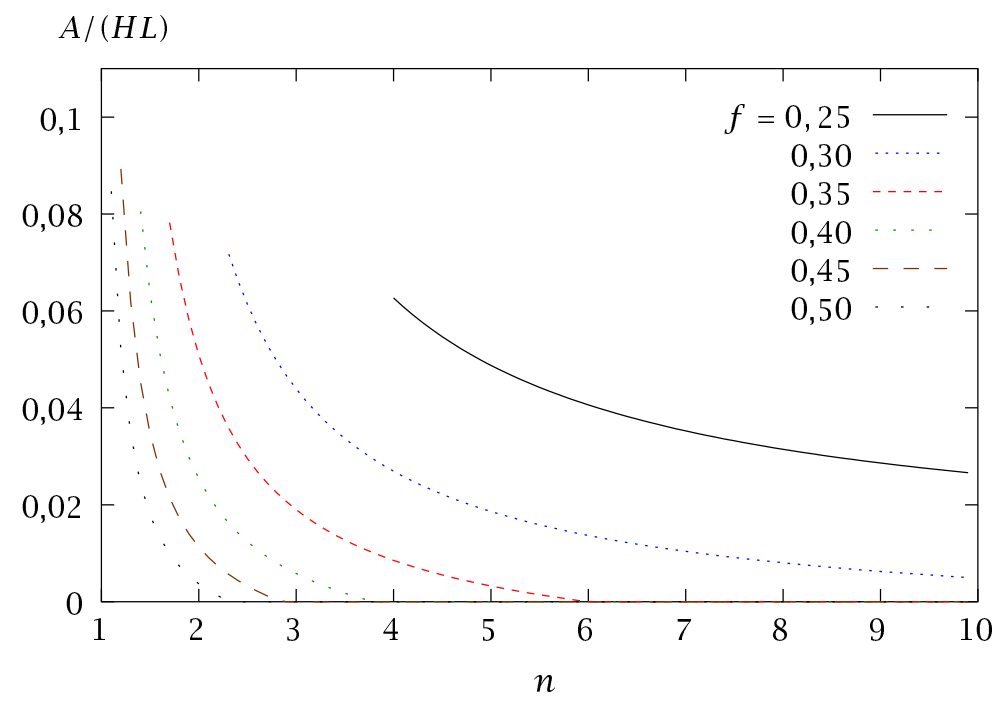

Figura 2.13: Área adimensional ocupada por el aire en la cámara de inyección en $t=t_{c}$, en función de $n$, para una ley de aceleración potencial en la que $x_{c}=L$.

ración exponencial, para la que el valor óptimo de $\xi$ será mayor que $4 / 3$, un valor de $n$ más grande que la unidad produciría perfiles de ola más verticales en $t_{c}$ que los obtenidos con $n=1$, haciendo posible reducir el volumen de aire en la cámara en ese instante y, por lo tanto, el volumen de aire finalmente atrapado en el metal, aunque un valor de $n>1$ tenderá a hacer que se refleje la ola en la pared final de la cámara antes del instante $t_{c}$.

De un estudio más detallado para diferentes valores de $\xi$ y $n$, se puede concluir que la ley de aceleración exponencial es normalmente preferible que la correspondiente a la Ecuación (2.43). A modo de ejemplo, se han representado en las Figuras 2.14a y 2.14b, para fracciones iniciales de llenado 0,25 y 0,5, respectivamente, los perfiles de la ola para los 
parámetros de las leyes de aceleración de las Ecuaciones (2.16) y (2.43) que satisfacen la condición $x_{c}=L$ y minimizan el tiempo de llenado $t_{f}$ (véanse las Figuras 2.6 y 2.11). Para la ley exponencial, los tiempos de llenado mínimos que satisfacen la condición anterior son $t_{f} \mathcal{C}_{0} / L=1,066$ y 1,013 para $f=0,25$ y 0,5 , respectivamente, y para la ley potencial, $t_{f} \mathcal{C}_{0} / L=1,224$ y 1,066 para $f=0,25$ y 0,5 , respectivamente. ${ }^{\dagger}$ Se puede observar, de los últimos perfiles representados, correspondientes al instante de rotura $t_{c} \mathcal{C}_{0} / L$, que la ley potencial produciría una mayor cantidad de aire atrapado que la ley exponencial, requiriendo, además, tiempos de llenado superiores. En capítulos posteriores se analizarán en profundidad los efectos de reflexión de la ola contra la pared final de la cámara de inyección y la influencia de los efectos viscosos y no hidrostáticos en el movimiento del metal fundido.

\subsection{Ley óptima de movimiento del pistón}

Se ha demostrado que ninguna de las leyes de aceleración consideradas hace posible eliminar el aire de la cámara de inyección antes de que la ola empiece a romperse o reflejarse en la pared final de la cámara. Es posible obtener una ley de aceleración óptima a partir de la Ecuación (2.8) si se impone que el perfil de la ola sea totalmente vertical en $x=L$. Esta condición asegura que no exista rotura ni reflexión de la ola durante el proceso de llenado, y que el volumen de aire en la cámara de inyección, en el instante en el que se forma la discontinuidad, sea igual a cero.

Sustituyendo $t$ por $t_{c}$ y $x$ por $x_{c}$ en la Ecuación (2.8) se obtiene

$$
X^{\prime}\left(t_{1}\right)+\frac{2}{3} \frac{X\left(t_{1}\right)}{t_{c}-t_{1}}=\frac{2}{3}\left(\frac{x_{c}}{t_{c}-t_{1}}-c_{0}\right),
$$

lo que indica que todas las características positivas procedentes de los puntos $\left(X\left(t_{1}\right), t_{1}\right)$, siendo $t_{1} \leq t_{H}$, se cruzan en el punto crítico $\left(x_{c}, t_{c}\right)$. En la Figura 2.15 se puede ver esquemáticamente la evolución de las características para satisfacer la condición anterior. Con la condición de que $X(t)=0$ en $t=0$, la solución de la Ecuación (2.57) proporciona la localización de la superficie del pistón en función del tiempo

$$
X(t)=3 x_{c}\left[1-\left(1-\frac{t}{t_{c}}\right)^{2 / 3}\right]-2 c_{0} t .
$$

Si se introduce la condición de que la discontinuidad se forme justo en una posición $x_{c}=\ell$, entonces $t_{c}=\ell / c_{0}$, y la Ecuación (2.58) puede escri-

\footnotetext{
${ }^{\dagger}$ Obsérvese que con los parámetros elegidos, la ola empezará a romperse en una posición intermedia entre el extremo más avanzado y la parte superior del frente de la ola, por lo que ésta empezará a reflejarse contra la pared final de la cámara en un instante anterior a $t_{c}\left(t=L / c_{0}\right)$.
} 


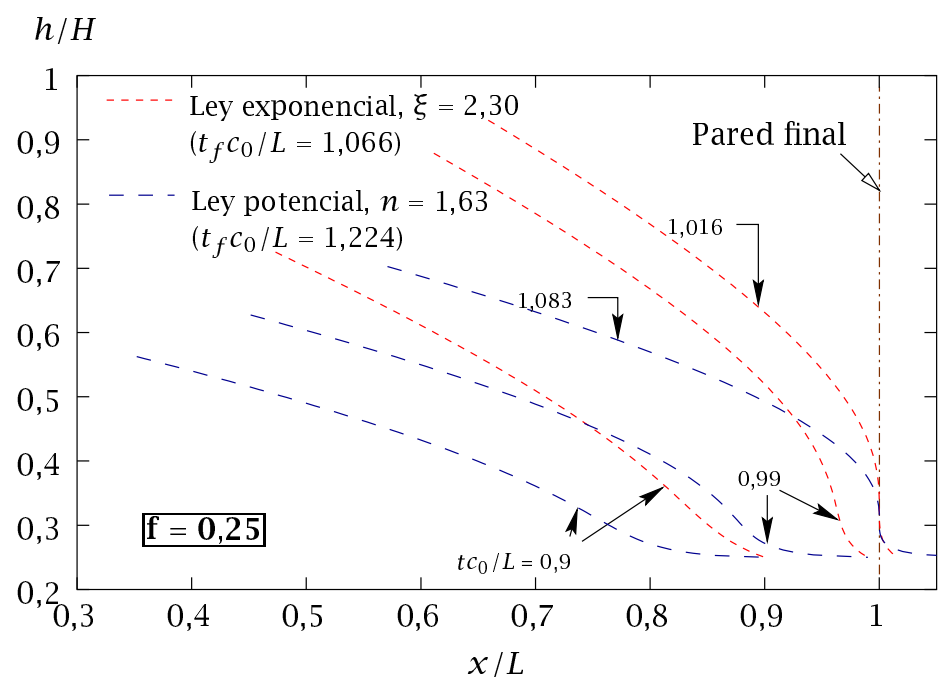

a)

$h / H$

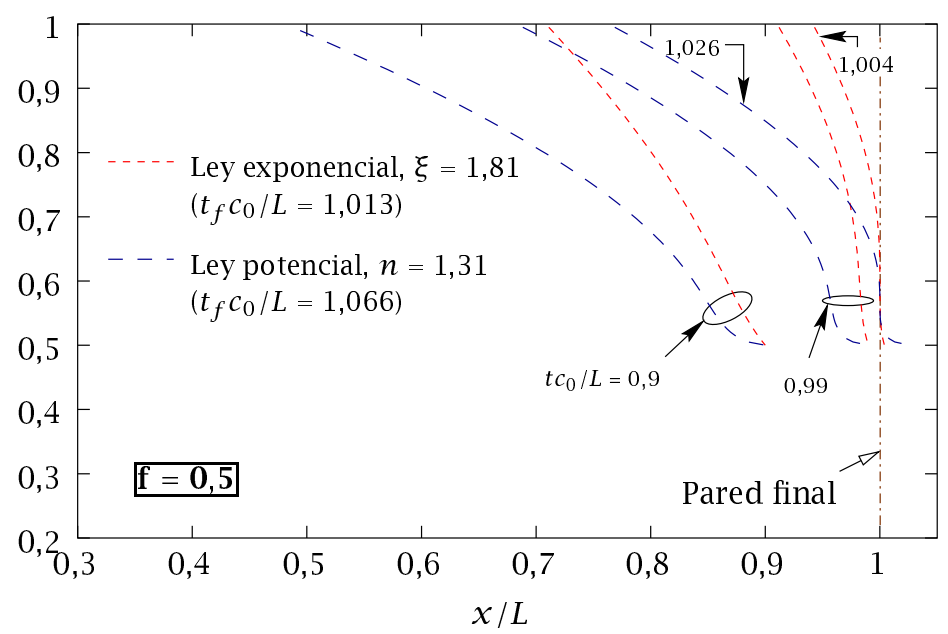

b)

Figura 2.14: Perfiles de la ola para los parámetros de las leyes de aceleración de las Ecuaciones (2.16) y (2.43) que satisfacen la condición $x_{c}=L$ y minimizan el tiempo de llenado $t_{f}$. a) $f=0,25$. b) $f=0,5$. 


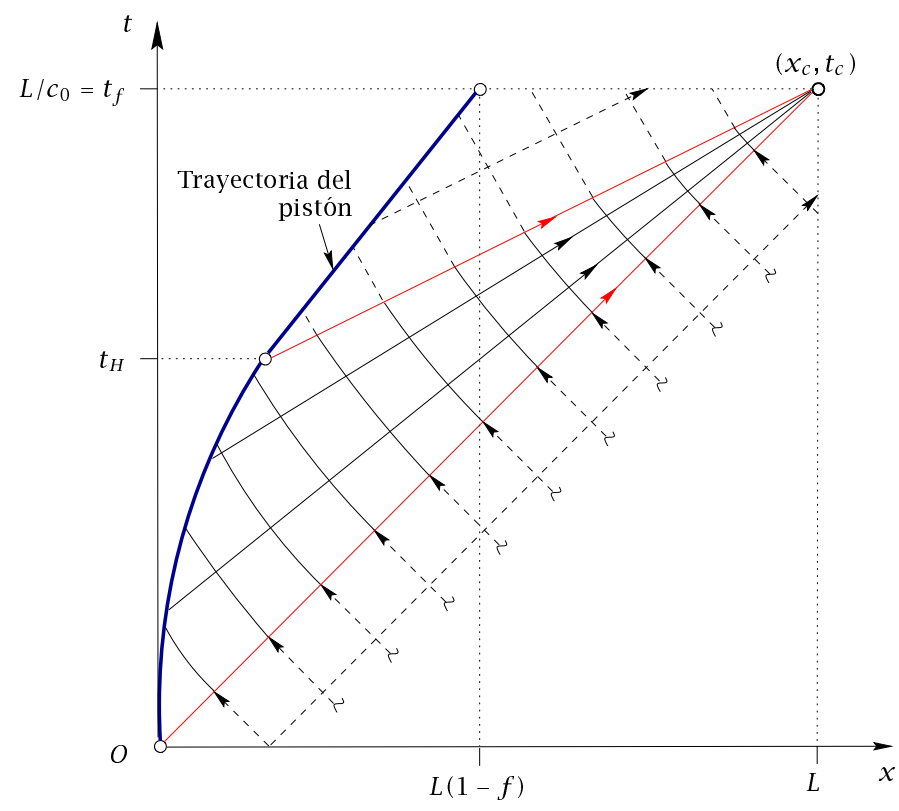

Figura 2.15: Esquema de la evolución de las características para que el perfil de la ola sea completamente vertical en $x=x_{c}=L$. 
birse de la forma

$$
X(t)=3 \ell\left[1-\left(1-\frac{c_{0} t}{\ell}\right)^{2 / 3}\right]-2 c_{0} t .
$$

Las leyes de velocidad y aceleración correspondientes son por tanto

$$
\begin{gathered}
X^{\prime}(t)=2 c_{0}\left[\left(1-\frac{c_{0} t}{\ell}\right)^{-1 / 3}-1\right], \\
X^{\prime \prime}(t)=\frac{2 c_{0}^{2}}{3 \ell}\left(1-\frac{c_{0} t}{\ell}\right)^{-4 / 3} .
\end{gathered}
$$

Obviamente, cuando $\ell$ es igual a la longitud de la cámara de inyección, $L$, la ley de aceleración de la Ecuación (2.61) producirá un perfil de ola completamente vertical en la pared final de la cámara de inyección en el instante $t=t_{c}=L / c_{0}$, por lo que todo el volumen de aire inicial será expulsado antes de ser atrapado.

Se puede deducir fácilmente que el instante en el que el metal fundido alcanza la altura máxima de la superficie libre $\eta$ en la cara del pistón y cesa la aceleración del pistón es

$$
t_{\eta}=\frac{\ell}{c_{0}}\left[1-\left(h_{0} / \eta\right)^{3 / 2}\right],
$$

por lo que el metal fundido alcanzará el techo de la cámara de inyección en

$$
t_{\eta=H}=\frac{L}{c_{0}}\left(1-f^{3 / 2}\right),
$$

de donde se deduce que $t_{H}$ tiende a $t_{c}$ cuando $f \rightarrow 0$. Para $t>t_{H}$ la velocidad del pistón se debería mantener constante.

Introduciendo las Ecuaciones (2.59) y (2.60) para $\ell=L$ en las Ecuaciones (2.8) y (2.9), se puede obtener el perfil de la ola en función del tiempo para valores de $h_{0}$ y $L$ dados:

$$
\frac{x}{L}=1+\left[2-3\left(\frac{h}{h_{0}}\right)^{1 / 2}\right]\left[1-\frac{\left(g h_{0}\right)^{1 / 2}}{L} t\right] .
$$

La evolución del perfil de la ola se presenta en la Figura 2.16 para diferentes valores de la fracción inicial de llenado. Se puede observar que el perfil de la ola es totalmente vertical en $t / t_{c}=1$. Además, con esta ley de movimiento la duración de la fase lenta de inyección se minimiza, haciendo el tiempo de llenado igual a $t_{c}$, que obviamente decrece conforme la fracción inicial de llenado aumenta.

Como ya se ha mencionado, para evitar un aumento del volumen de aire atrapado, la ola de metal fundido debe alcanzar el techo de la cámara 

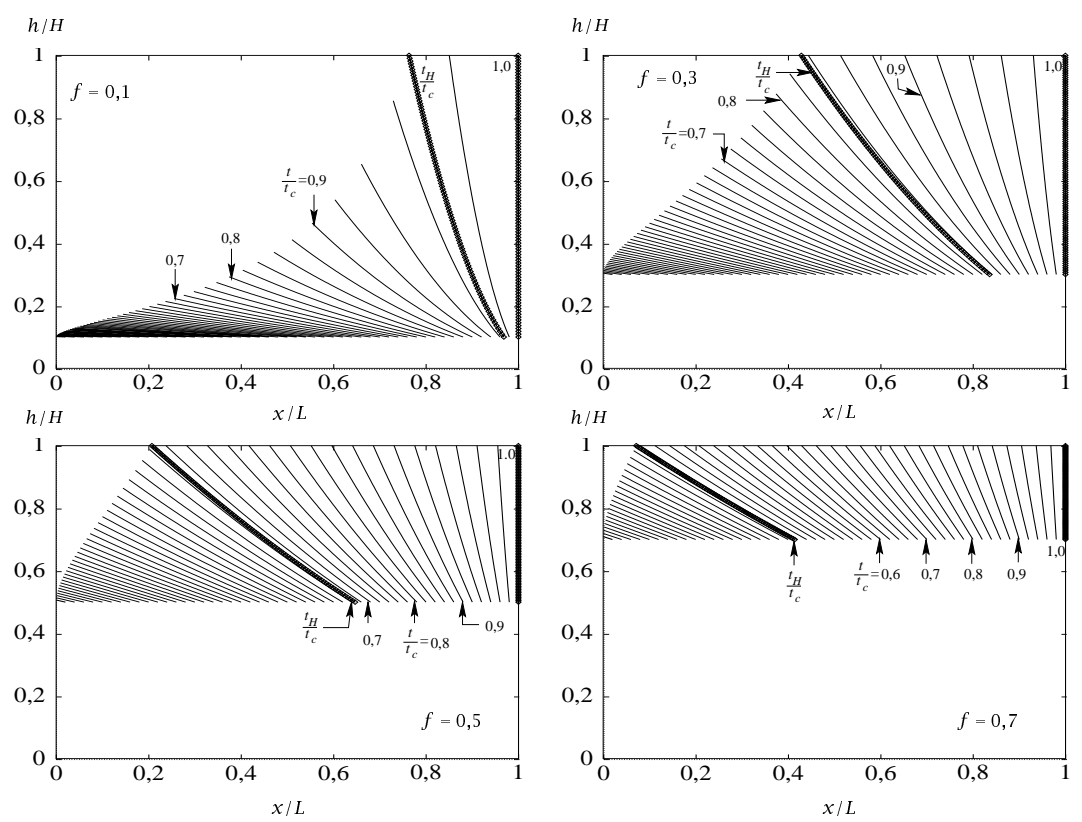

Figura 2.16: Evolución del perfil de la ola con $t / t_{c}$ para diferentes valores de la fracción inicial de llenado y la ley de aceleración óptima del pistón de la Ecuación (2.61) para $\ell=L$. 
de inyección antes de que ésta empiece a romperse. Tal y como se expuso en las Secciones 2.2.2 y 2.3, las leyes de aceleración exponencial y potencial en el tiempo precisan una fracción inicial de llenado mínima, que depende de $\xi$ y $n$ respectivamente, para satisfacer la condición anterior. Una de las ventajas de la ley de aceleración de la Ecuación (2.61) es precisamente que no requiere un valor mínimo de la fracción inicial de llenado para asegurar que la velocidad del pistón alcance la velocidad crítica antes de $t_{c}$. Sin embargo, esta ley impone un requerimiento más restrictivo con respecto a la fracción inicial de llenado máxima que puede utilizarse, tal y como se discutirá a continuación.

\subsection{Límite máximo de la fracción inicial de llena- do}

En los procesos de fundición por inyección a alta presión en cámaras frías, el metal fundido se vierte en la cámara de inyección, a mano o automáticamente, a través de un orificio de colada. Aunque en el Capítulo 4 se analizará numéricamente la influencia de los efectos no hidrostáticos sobre la evolución de la ola en la cámara de inyección, en la Figura 2.17 se adelantan algunos resultados correspondientes a un caso en el que la velocidad del pistón se mantiene constante e igual al valor dado por

$$
u_{\eta}=2\left(\sqrt{g \eta}-\sqrt{g h_{0}}\right),
$$

para $t \geq t_{\eta}$ con $\eta<H$. Se puede observar que, debido a estos efectos, la superficie libre de la ola alcanza una altura superior a $\eta$. Teniendo esto en cuenta, para impedir que el metal fundido salga fuera de la cámara de inyección a través del orificio de colada cuando $\eta=H$, se debe asegurar que la ola no alcance el techo de la cámara de inyección antes de que el pistón cierre dicho orificio. Esta condición se puede expresar de la forma siguiente:

$$
X\left(t_{H}\right) \geq X_{p},
$$

donde $X_{p}$ es la distancia que debe recorrer el pistón hasta alcanzar el extremo final del orificio de colada. En la Figura 2.18 se representa un esquema de la cámara de inyección en la que se puede ver la localización del orificio de colada y la posición límite de la superficie del pistón en $t=t_{H}$ para satisfacer la condición de la Ecuación (2.66).

Sustituyendo la Ecuación (2.63) en la expresión de $X(t)$ correspondiente a la ley de aceleración de la Ecuación (2.61) para $\ell=L$ e imponiendo la condición de la Ecuación (2.66), se obtiene la siguiente relación para el límite superior de la fracción inicial de llenado, $f_{\max }$ :

$$
\frac{X_{p}}{L}=1-3 f_{\max }+2 f_{\max }^{3 / 2} \text {. }
$$




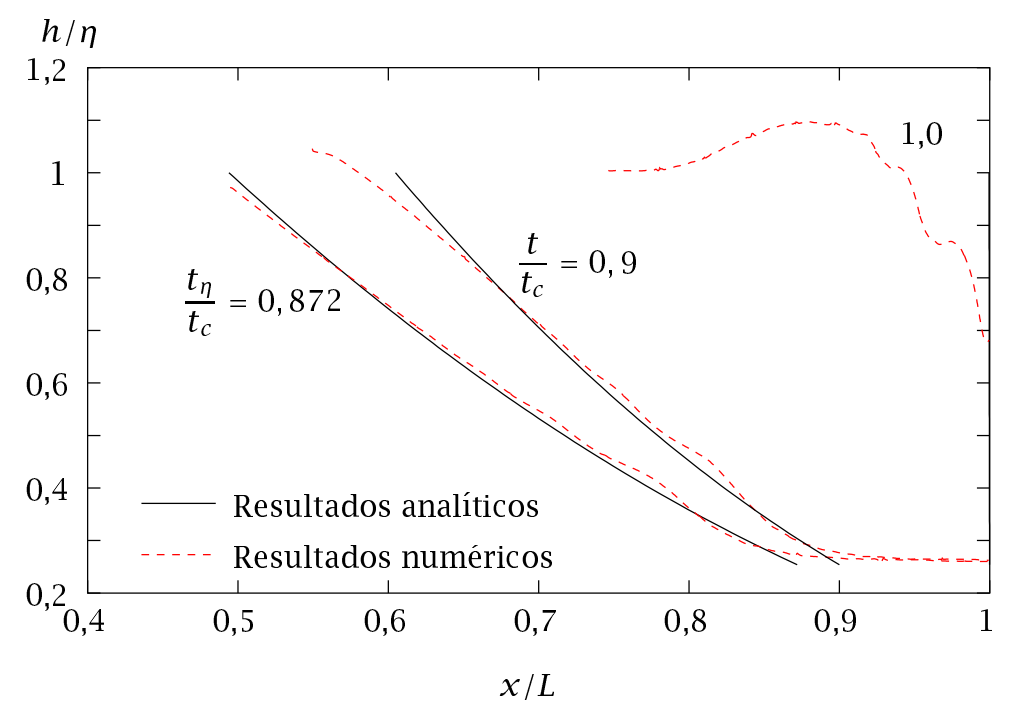

Figura 2.17: Comparación entre los perfiles de la superficie de la ola obtenidos analíticamente y los obtenidos numéricamente para la ley de aceleración de la Ecuación (2.61) con $\ell=L$ y $X^{\prime}=u_{\eta}$ para $t>t_{\eta} . \eta=5,08 \mathrm{~cm}, H / \eta=1,3, f=0,254$ $\mathrm{y} \ell / \eta=9$. 

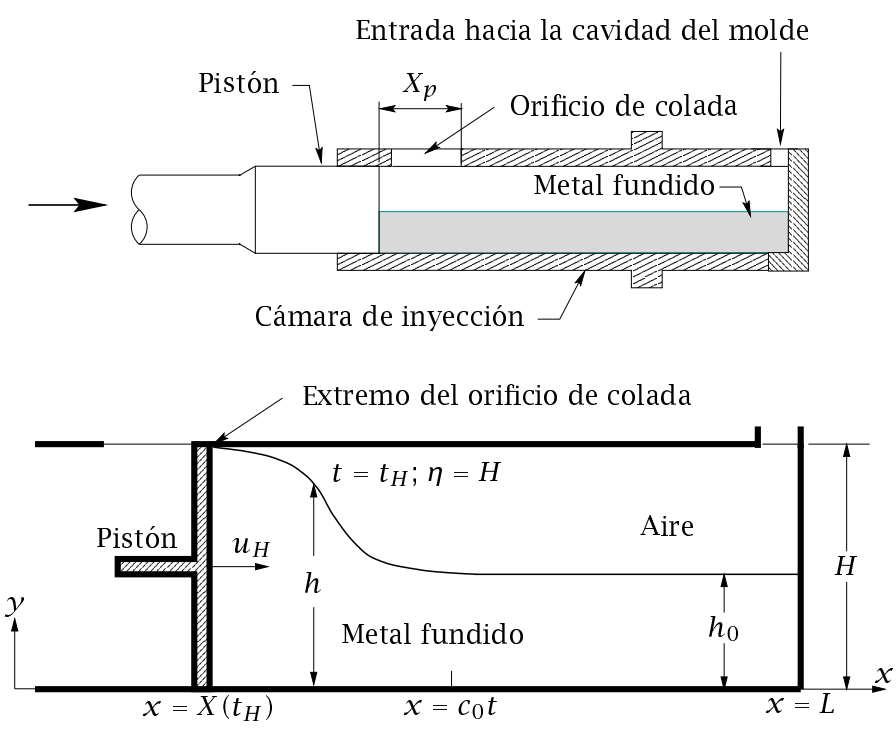

Figura 2.18: Esquema de la cámara de inyección.

Para la ley de aceleración exponencial se puede deducir una expresión equivalente a la Ecuación (2.67). Sustituyendo la Ecuación (2.29) en la expresión para $X(t)$ correspondiente a la ley de aceleración de la Ecuación (2.16), la condición de la Ecuación (2.66) se puede escribir como:

$$
\frac{X_{p}}{L} \leq \frac{\beta}{L}\left\{3 \xi\left(f^{-1 / 2}-1\right)-\ln \left[3 \xi\left(f^{-1 / 2}-1\right)+1\right]\right\} .
$$

Como se expuso anteriormente, la relación $\beta / L$ para las condiciones que hacen que la ola empiece a romperse en la pared final de la cámara de inyección se puede obtener imponiendo $x\left(t_{c}\right)=L$ y las condiciones de las Ecuaciones (2.11) y (2.12) en la Ecuación (2.8). Introduciendo esta relación en la Ecuación (2.68), se obtienen las siguientes relaciones entre la fracción inicial de llenado máxima y $X_{p} / L$. Para $\xi \leq \frac{4}{3}$, con la relación $\beta / L$ de la Ecuación (2.35) (la condición de la Ecuación (2.12) se debe reemplazar por $\left.t_{1}=0\right)$ :

$$
\frac{X_{p}}{L}=2 \xi^{-1}\left(f_{\max }^{-1 / 2}-1\right)-\frac{2}{3} \xi^{-2} \ln \left[3 \xi\left(f_{\max }^{-1 / 2}-1\right)+1\right] .
$$

Para $\xi>\frac{4}{3}$ y $f \leq f^{*}=\{9 \xi /[4(3 \xi-1)]\}^{2}$, con la relación $\beta / L$ de la 
Ecuación (2.33):

$$
\frac{X_{p}}{L}=\frac{3 \xi\left(f_{\max }^{-1 / 2}-1\right)-\ln \left[3 \xi\left(f_{\max }^{-1 / 2}-1\right)+1\right]}{5 \xi-4-\ln \left(\xi-\frac{1}{3}\right)} .
$$

Para $\xi>\frac{4}{3}$ y $f>f^{*}$, con la relación $\beta / L$ de la Ecuación (2.34) (la condición de la Ecuación (2.12) se debe reemplazar en este caso por $t_{1}=t_{H}$ ):

$$
\frac{X_{p}}{L}=\left(\frac{\frac{3}{2} \xi^{2} f_{\max }^{-1 / 2}\left(3 f_{\max }^{-1 / 2}-2\right)}{\left[3 \xi\left(f_{\max }^{-1 / 2}-1\right)+1\right]\left\{3 \xi\left(f_{\max }^{-1 / 2}-1\right)-\ln \left[3 \xi\left(f_{\max }^{-1 / 2}-1\right)+1\right]\right\}}+1\right)^{-1} .
$$

La Figura 2.19 muestra la fracción inicial de llenado máxima en función de $X_{p} / L$ para la ley de aceleración de la Ecuación (2.61) con $\ell=L \mathrm{y}$ para la ley exponencial con diferentes valores del parámetro $\xi$. Se pue-

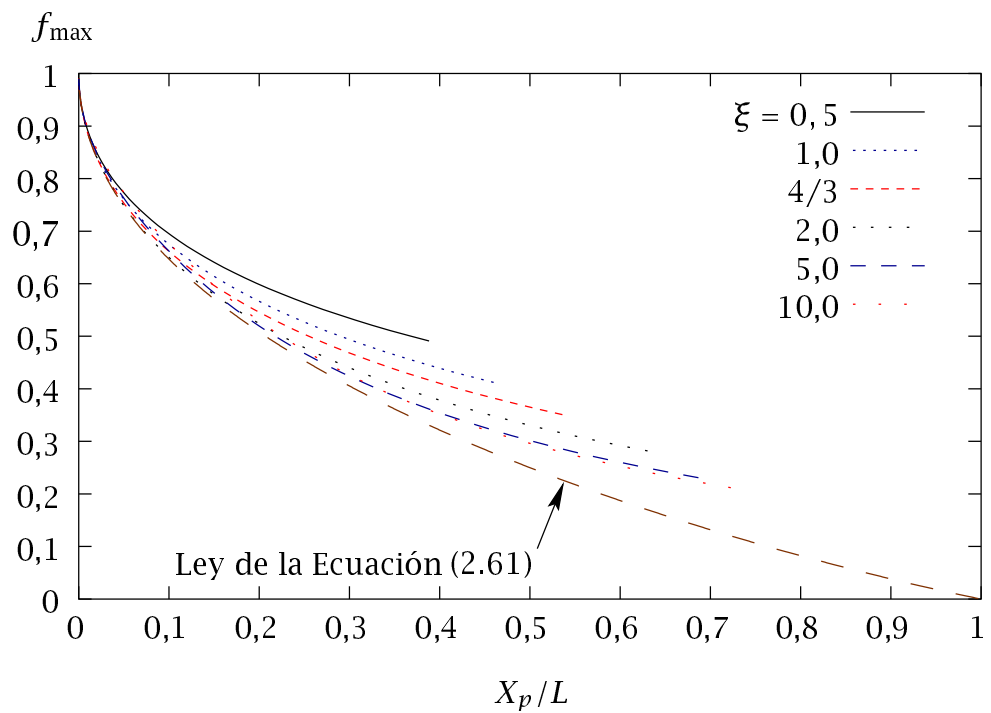

Figura 2.19: Fracción inicial de llenado máxima en función de $X_{p} / L$, para la ley aceleración exponencial y diferentes valores de $\xi$. Comparación con la ley de aceleración de la Ecuación (2.61).

de observar que la fracción inicial de llenado máxima para la nueva ley de aceleración es siempre más pequeña que para la ley exponencial para cualquier valor de la relación $X_{p} / L$. Se debería indicar que, para la 
ley exponencial, existe un valor límite de $X_{p} / L$, que aumenta conforme $\xi$ aumenta, por encima del cual $f_{\max }$ es menor que la fracción inicial de llenado mínima necesaria para que la ola alcance el techo de la cámara de inyección antes de romper. En la Figura $2.19, f_{\max }$ no se ha representado para valores de $X_{p} / L$ por encima de este límite.

De forma análoga, para la ley de aceleración potencial en el tiempo, la condición de la Ecuación (2.66) se puede expresar como:

$$
\frac{X_{p}}{L} \leq \frac{c_{0}^{(n+1) / n}}{L \gamma^{1 / n}} \frac{1}{n+1}\left[2\left(f^{-1 / 2}-1\right)\right]^{(n+1) / n},
$$

y las ecuaciones correspondientes a las Ecuaciones (2.69), (2.70) y (2.71) son en este caso las siguientes. Para $n=1$, con el parámetro $L \gamma^{1 / n} / c_{0}^{(n+1) / n}$ de la Ecuación (2.54):

$$
\frac{X_{p}}{L}=3\left(f_{\max }^{-1 / 2}-1\right)^{2} .
$$

Para $n>1$ y $f \leq f^{*}=[(3 n+1) / 4 n]^{2}$, con el parámetro $L \gamma^{1 / n} / c_{0}^{(n+1) / n}$ de la Ecuación (2.50):

$$
\frac{X_{p}}{L}=\frac{(n-1)^{(n-1) / n}}{5 n^{2}+\frac{1}{3}(2 n-1)}\left[(3 n+1)\left(f_{\max }^{-1 / 2}-1\right)\right]^{(n+1) / n} .
$$

Para $n>1$ y $f>f^{*}$, con el parámetro $L \gamma^{1 / n} / c_{0}^{(n+1) / n}$ de la Ecuación (2.51):

$$
\frac{X_{p}}{L}=\frac{1}{1+\frac{n+1}{3 n} f_{\max }^{-1 / 2}\left(\frac{3}{2} f_{\max }^{-1 / 2}-1\right)\left(f_{\max }^{-1 / 2}-1\right)^{-2}} .
$$

La Figura 2.20 muestra la fracción inicial de llenado máxima en función de $X_{p} / L$ para la ley de aceleración de la Ecuación (2.61) con $\ell=L$ y para la ley potencial con diferentes valores de $n$. De nuevo, se puede observar que $f_{\max }$ para la nueva ley es siempre menor que para la ley potencial. Por lo tanto, la nueva ley es más restrictiva que las leyes exponencial y potencial con respecto a la fracción inicial de llenado máxima que se puede usar para evitar que el metal fundido sea expulsado de la cámara de inyección a través del orificio de colada. Se puede observar que para la ley potencial existe un valor límite de $X_{p} / L$, que aumenta conforme $n$ aumenta, por encima del cual $f_{\max }$ es menor que la fracción inicial de llenado mínima necesaria para que la ola alcance el techo de la cámara de inyección antes de que comience a romperse.

Las variables del proceso de la fase lenta se tendrán que elegir teniendo en cuenta, entre otros parámetros, la fracción inicial de llenado necesaria para fabricar la pieza deseada y la distancia inicial entre la superficie del pistón y el extremo final del orificio de colada, $X_{p}$. Para facilitar esta selección, la fracción inicial de llenado máxima, $f_{\max }$, se ha 


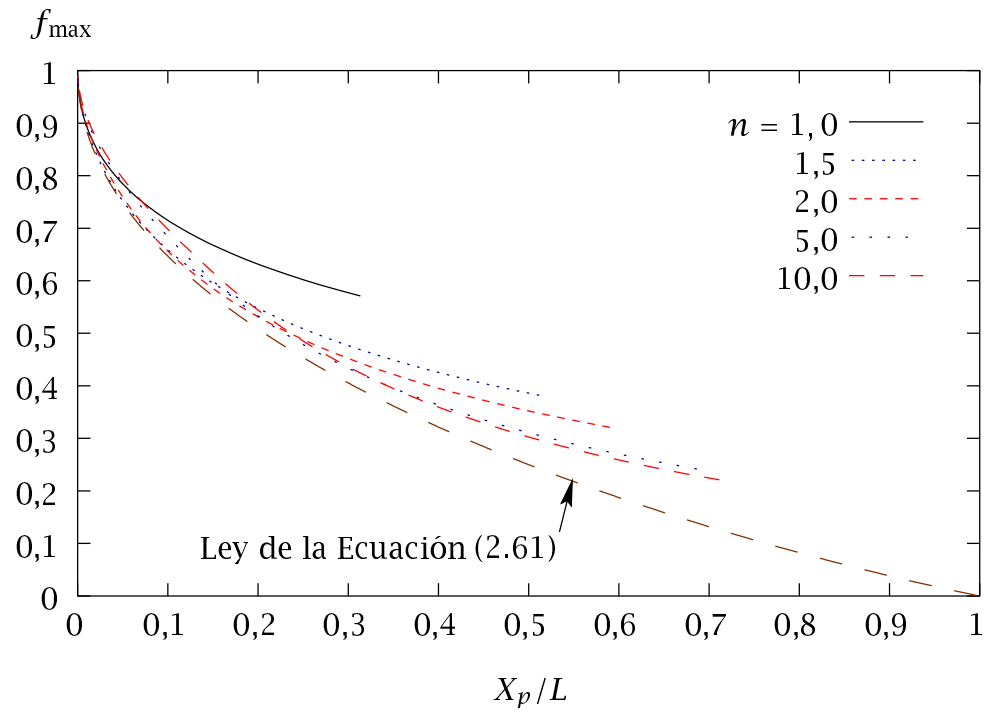

Figura 2.20: Fracción inicial de llenado máxima en función de $X_{p} / L$, para la ley de aceleración potencial y diferentes valores de $n$. Comparación con la ley de aceleración de la Ecuación (2.61). 
representado en las Figuras 2.21 y 2.22 en función de $\xi$ para la ley exponencial y en función de $n$ para la ley potencial, para valores de $X_{p} / L$ comprendidos entre 0,1 y 0,3 satisfaciendo la condición $x_{c}=L$. Por

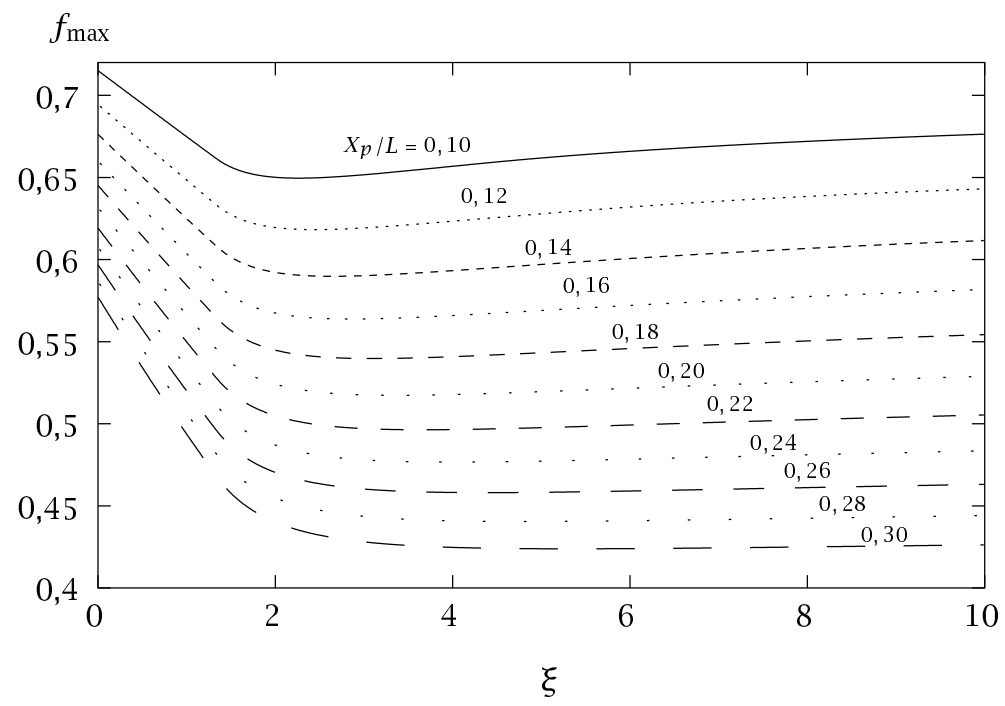

Figura 2.21: Fracción inicial de llenado máxima en función de $\xi$, para diferentes valores de $X_{p} / L$, para la ley de aceleración exponencial.

ejemplo, supongamos una cámara de inyección con una relación $X_{p} / L$ igual a 0,1 y una fracción inicial de llenado de 0,66 . Se puede deducir de la Figura 2.21 que el metal fundido podría ser expulsado de la cámara de inyección a través del orificio de colada si se usa una ley de aceleración exponencial con valores de $\xi$ comprendidos entre 1,375 y 4,608. Para el mismo caso con la ley potencial (Figura 2.22), se puede ver que se deben evitar valores de $n$ comprendidos entre 1,454 y 2,402. En este caso, la nueva ley de aceleración, para la que la fracción inicial de llenado máxima es 0,647 , no es apropiada. No obstante, las limitaciones más restrictivas de la ley de aceleración de la Ecuación (2.61) respecto a la fracción inicial de llenado máxima para la que se puede emplear se ven compensadas por los menores tiempos de llenado necesarios, la reducción esperada de los efectos del aire atrapado y la posibilidad de usar fracciones iniciales de llenado más pequeñas.

Aunque los efectos viscosos y no hidrostáticos influirán en alguna medida, especialmente bajo ciertas circunstancias, en el flujo del metal fundido en la cámara de inyección, la ley de aceleración óptima, obtenida 


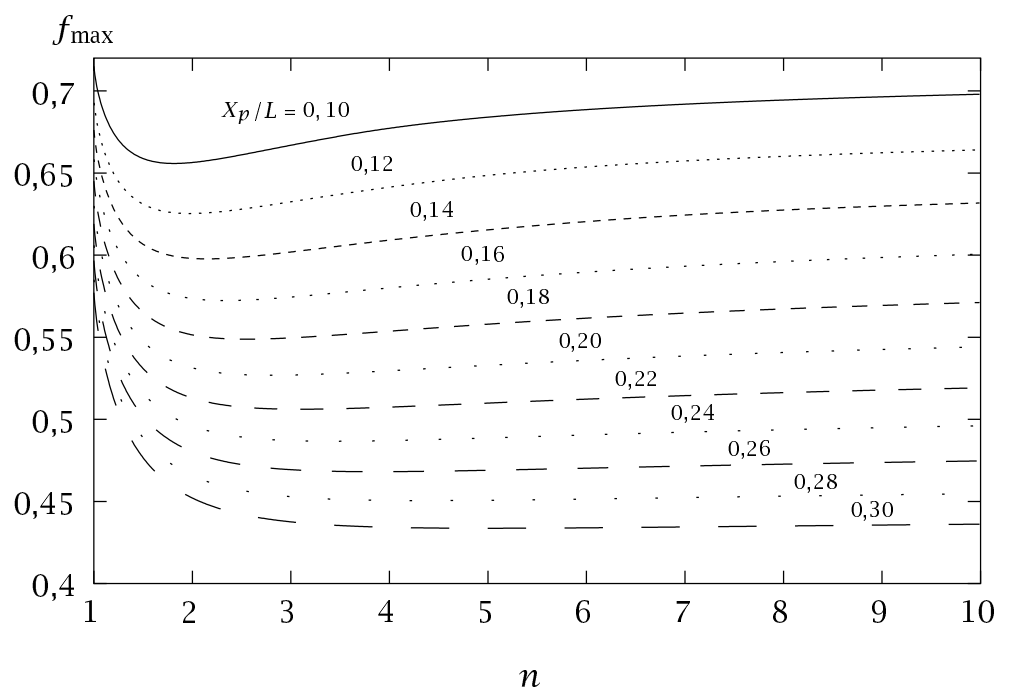

Figura 2.22: Fracción inicial de llenado máxima en función de $n$, para diferentes valores de $X_{p} / L$, para la ley de aceleración potencial. 
despreciando estos efectos, y el análisis del movimiento de la ola presentado en este capítulo, puede ser de ayuda en la elección de las variables que optimicen el proceso. En el Capítulo 4 se realizará una investigación numérica para analizar la influencia de estos efectos en la evolución de la ola dentro de la cámara de inyección.

\subsection{Conclusiones}

Se ha presentado un modelo analítico, basado en la aproximación de aguas poco profundas, para estudiar el movimiento del metal fundido en la fase lenta de los procesos de fundición por inyección a alta presión. Se ha llevado a cabo un análisis de los posibles perfiles de ola de metal fundido para diferentes leyes de aceleración y amplios rangos de los parámetros relevantes del proceso, para los que se determina el lugar e instante en el que se formará una discontinuidad en la superficie del metal líquido. Se han obtenido diferentes relaciones adimensionales entre los parámetros que caracterizan la ley de aceleración aplicada al pistón, la fracción inicial de llenado y las características geométricas de la cámara de inyección, que hacen posible elegir de forma apropiada las variables del proceso que minimicen el aire atrapado, reduciendo de este modo la porosidad de las piezas fabricadas, y mantengan el tiempo de llenado tan pequeño como sea posible. También se han determinado los rangos de los parámetros de proceso que se deberían evitar.

Para la ley de aceleración exponencial en el tiempo se ha deducido que el valor óptimo del parámetro $\xi$ debe ser algo mayor a $4 / 3$. Sería necesario un análisis detallado de los procesos de atrapamiento de aire causados por la rotura y reflexión de la ola en la cámara de inyección para una determinación más precisa de este óptimo. Se ha encontrado que se necesita una fracción inicial de llenado mínima para evitar que la rotura de la ola se produzca antes de que el metal fundido alcance el techo de la cámara de inyección, la cual es una función decreciente en $\xi$ con un máximo de 9/16 en el límite $\xi \rightarrow 0$ (número de Froude infinito) y $f_{\min }=0,195$ cuando $\xi \rightarrow \infty$. Se han obtenido resultados similares para la ley de aceleración potencial. Por otro lado, la ley exponencial produce perfiles de ola más inclinados en $t=t_{c}$ que los de la ley potencial, por lo que se espera que reduzca el volumen de aire atrapado.

Las predicciones obtenidas del parámetro de aceleración $\alpha$ en función de la fracción inicial de llenado se han comparado con los resultados de Tszeng y Chu (1994), obteniendo una concordancia cualitativamente excelente, a pesar de que los valores del modelo presentado en este capítulo son ligeramente inferiores. Los resultados del modelo propuesto también se compararon con los obtenidos experimentalmente por Duran et al. (1991), obteniendo de nuevo una buena concordancia a pesar de las 
incertidumbres existentes en la comparación.

Se ha observado que ninguna de las leyes de aceleración consideradas hace posible obtener un conjunto de factores que elimine totalmente el aire del interior de la cámara de inyección en el momento en el que la ola alcanza la pared final. Para eliminar estas limitaciones, se ha deducido analíticamente una ley de aceleración que hace posible obtener un perfil de la ola totalmente vertical cuando alcanza la pared final de la cámara de inyección para cualquier valor de la fracción inicial de llenado, y minimiza adicionalmente el tiempo de llenado en la fase lenta. Por otro lado, para que el metal fundido no sea expulsado fuera de la cámara de inyección a través del orificio de colada, se ha encontrado que la nueva ley introduce, con respecto a la máxima fracción inicial de llenado que se puede usar, restricciones ligeramente superiores a las de otras leyes de movimiento habitualmente usadas en los procesos de fundición por inyección a alta presión. 



\section{Efectos de reflexión de la ola de metal fundido en la cámara de inyección}

En el estudio analítico del movimiento del metal fundido realizado en el Capítulo 2 mediante el modelo basado en la aproximación de aguas poco profundas, se despreciaron los efectos de reflexión de la ola contra la pared final de la cámara de inyección. En este capítulo, el mismo modelo anterior se resuelve numéricamente teniendo en cuenta estos efectos, utilizando para ello el método de las características. Para situaciones en las que los efectos de reflexión puedan ser importantes, se obtienen de esta forma los perfiles de la ola y los valores óptimos de los parámetros que caracterizan la ley de aceleración del pistón y se comparan con los del modelo analítico sin reflexión. Asimismo, se obtiene la cantidad de aire residual en la cámara de inyección en el instante en el que el metal fundido alcanza la entrada hacia la cavidad del molde. Los resultados serán comparados en el Capítulo 4 con los obtenidos utilizando un modelo numérico en el que se retendrán los efectos viscosos y no hidrostáticos.

\subsection{Modelo analítico con efectos de reflexión}

Tal y como se expuso en el capítulo anterior, mediante la aproximación de aguas poco profundas y aplicando el método de las características 
(véase el Apéndice A.1), las ecuaciones del problema se pueden reducir a las ecuaciones de compatibilidad

$$
u \pm 2 c=\text { constante, }
$$

a lo largo de las líneas características

$$
\frac{\mathrm{d} x}{\mathrm{~d} t}=u \pm c
$$

Las Ecuaciones (3.1) y (3.2) se resolverán imponiendo como condición de contorno adicional a la impuesta en la superficie del pistón el que la componente horizontal de la velocidad del metal fundido, $u$, sea cero en la posición $x=L$ correspondiente a la pared final de la cámara de inyección. En la Figura 3.1 se representa un esquema ilustrativo de las curvas características considerando los efectos de la reflexión del metal en la pared final de la cámara. Se han representado con líneas de trazos discontinuos las características positivas que parten de los puntos $(0,0)$ y $\left(X\left(t_{H}\right), t_{H}\right)$. A continuación se analizarán cada una de las regiones de flujo que aparecen representadas en el esquema de la Figura 3.1.

Región de ondas simples $(O B C E)$. Como en el modelo del capítulo anterior, en $t=0$ el fluido tiene una profundidad uniforme, $h_{0}$, y está en reposo, y el pistón empieza a moverse en el sentido positivo de $x$ con velocidad $X^{\prime}(t)$. En la región sin perturbar, es decir, la región que aún no ha sido alcanzada por la característica positiva inicial originada en $t=0$ en la superficie del pistón, todas las características son líneas rectas con pendiente $\pm 1 / c_{0}$. Estas características deben satisfacer la condición $u \pm 2 c= \pm 2 c_{0}$. La curva $x=c_{0} t$, correspondiente a la característica inicial, establece el límite de la región anterior, por encima de la cual la condición de contorno $u=X^{\prime}(t)$, impuesta en la superficie del pistón, determina que la condición de flujo uniforme de la región sin perturbar, $u=0$ y $c=c_{0}$, no se mantenga. Las características que parten de la superficie del pistón entre los instantes $t=0 \mathrm{y} t=t_{H}$ forman una región de ondas simples en la que las características negativas deben satisfacer la condición $u-2 c=-2 c_{0}$, ya que todas ellas proceden de la región sin perturbar.* De la Ecuación (3.1) se deduce que $u$ y $c$ son constantes a lo largo de las características positivas en esta región $\left(u=X^{\prime}\left(t_{1}\right)\right.$ y $\left.c=\frac{1}{2} X^{\prime}\left(t_{1}\right)+c_{0}\right)$, por lo que se deduce de la Ecuación (3.2) que estas características son líneas rectas cuya pendiente disminuye conforme aumenta el instante $t=t_{1}$ en el que son emitidas desde la superficie del pistón. La pendiente de las características negativas dependerá de las

*Recuérdese, que para reducir la cantidad de aire atrapado, suele hacerse que la aceleración del pistón cese en el instante $t_{H}$ en el que el pistón alcanza la velocidad crítica $u_{H}$. 


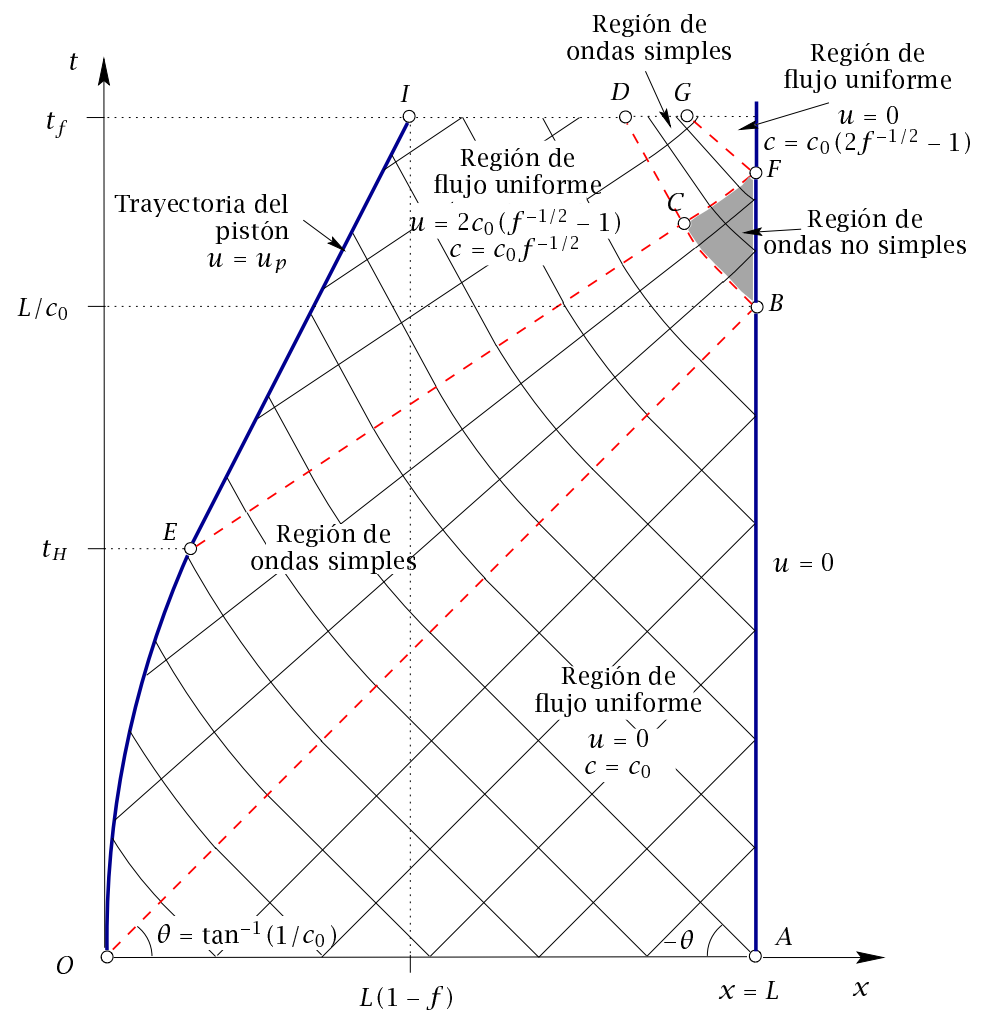

Figura 3.1: Esquema de la evolución de las características considerando los efectos de la reflexión del metal en la pared final de la cámara $(x=L)$. 
características positivas con las que se van cruzando, variando su valor desde $-1 / c_{0}$ a lo largo de la característica positiva originada en $t_{1}=0$, hasta el valor que se obtiene de las Ecuaciones (2.28) y (3.2) a lo largo de la característica positiva originada en $t_{1}=t_{H}$ :

$$
\left.\frac{\mathrm{d} t}{\mathrm{~d} x}\right|_{-}=\frac{1}{c_{0}\left(f^{-1 / 2}-2\right)} .
$$

En esta región, la profundidad del fluido junto a la superficie del pistón aumenta desde $h_{0}$ en $t=0$ hasta la altura total $H$ de la cámara de inyección en $t=t_{H}$.

Región de ondas no simples (BFC). La región de ondas simples anterior es adyacente a una región de ondas no simples (región $B F C$ ) en la que se cruzan las características positivas procedentes de la región de ondas simples y las correspondientes características reflejadas en la pared final de la cámara. La curva $B C$ que separa ambas regiones, dibujada en la Figura 3.1 con trazo discontinuo, corresponde a la característica inicial que se originó en $t_{1}=0$ en la superficie del pistón y que se refleja en la pared final de la cámara de inyección. Resulta inmediato determinar analíticamente esta curva, y para ello, la pendiente de las características positivas en la región de ondas simples $O B C E$ se expresa a partir de la Ecuación (3.2) del siguiente modo:

$$
\frac{t-t_{1}}{x-X\left(t_{1}\right)}=\frac{1}{\frac{3}{2} X^{\prime}\left(t_{1}\right)+c_{0}}
$$

( $t_{1}$ es el instante en el que se origina la característica en el pistón). Por otro lado, como se ha comentado anteriormente, en esta región de ondas simples la pendiente de las características negativas depende de las características positivas con las que se cruzan, y su valor puede ser expresado a partir de la Ecuación (3.2) en función de $t_{1}$, teniendo en cuenta que, de acuerdo con la Ecuación (3.1), $u-2 c=-2 c_{0}$, del siguiente modo:

$$
\left.\frac{\mathrm{d} t}{\mathrm{~d} x}\right|_{-}=\frac{1}{\frac{1}{2} X^{\prime}\left(t_{1}\right)-c_{0}} .
$$

Derivando la Ecuación (3.4) con respecto a $t_{1}$ resulta:

$$
\left[\frac{3}{2} X^{\prime}\left(t_{1}\right)+c_{0}\right] \frac{\mathrm{d} t}{\mathrm{~d} t_{1}}+\frac{3}{2} X^{\prime \prime}\left(t_{1}\right)\left(t-t_{1}\right)-\frac{1}{2} X^{\prime}\left(t_{1}\right)-c_{0}=\frac{\mathrm{d} x}{\mathrm{~d} t_{1}} .
$$

Por otra parte, la Ecuación (3.5) se puede expresar así:

$$
\left[\frac{1}{2} X^{\prime}\left(t_{1}\right)-c_{0}\right] \frac{\mathrm{d} t}{\mathrm{~d} t_{1}}=\frac{\mathrm{d} x}{\mathrm{~d} t_{1}} .
$$


Introduciendo la Ecuación (3.6) en la Ecuación (3.7), se obtiene

$$
\frac{\mathrm{d} t}{\mathrm{~d} t_{1}}+\frac{3}{2} \frac{X^{\prime \prime}\left(t_{1}\right)}{X^{\prime}\left(t_{1}\right)+2 c_{0}} t=\frac{3}{2} \frac{X^{\prime \prime}\left(t_{1}\right)}{X^{\prime}\left(t_{1}\right)+2 c_{0}} t_{1}+\frac{1}{2},
$$

cuya solución general puede ser expresada del siguiente modo:

$$
t=t_{1}-\frac{1}{\left[X^{\prime}\left(t_{1}\right)+2 c_{0}\right]^{3 / 2}}\{\frac{1}{2} \underbrace{\int\left[X^{\prime}\left(t_{1}\right)+2 c_{0}\right]^{3 / 2} \mathrm{~d} t_{1}}_{I}-K\},
$$

donde la integral $I$ se debe determinar para la correspondiente ley de movimiento y la constante $K$ se calcula imponiendo la condición $x=L$ en $t=L / c_{0}$ para $t_{1}=0$, lo que corresponde al punto $B$ representado en la Figura 3.1. La curva $B C$ queda determinada en forma paramétrica por las Ecuaciones (3.4) y (3.9) para $0 \leq t_{1} \leq t_{H}$. Por ejemplo, para la ley de aceleración del pistón de la Ecuación (2.16), la expresión de la curva $B C$ en forma paramétrica se puede expresar del siguiente modo

$$
x=X\left(t_{1}\right)+\left[\frac{3}{2} X^{\prime}\left(t_{1}\right)+c_{0}\right]\left(t-t_{1}\right)
$$

para $t_{1}$ comprendido entre 0 (punto $B$ ) y $t_{H}=\ln \left[3 \xi\left(f^{-1 / 2}-1\right)+1\right] / \alpha$ (punto $C$ ). En la Figura 3.2 se representa la curva $B C$ de las Ecuaciones (3.10) y (3.10) para la ley exponencial con diferentes fracciones iniciales de llenado, $f$, y distintos valores del parámetro $\xi$. Los parámetros de la ley de aceleración se han elegido para satisfacer, según el modelo analítico sin reflexión, la condición $x_{c}=L$ en $t=t_{c}$ para cada valor de $\xi$ (en $x=L$ se formaría una discontinuidad si se prescinde de los efectos de reflexión). El extremo izquierdo de cada una de estas curvas corresponde al punto $C$ representado en la Figura 3.1. Este punto determina el instante a partir del cual la cresta de la ola empezaría a alcanzar una profundidad superior a la altura total $H$ de la cámara de inyección (en ausencia de la pared superior de la cámara). Obsérvese, por ejemplo que, para fracciones iniciales de llenado $f=0,3$ y 0,5 , y valores de $\xi$ superiores a 6,141 y 2,631, respectivamente, la cresta de la ola empezaría a crecer por encima de $H$ antes del instante $t=t_{c}$.

En esta región de ondas no simples, en la que las características reflejadas en la pared final se cruzan con las características positivas que se originaron en la superficie del pistón entre $t_{1}=0$ y $t_{1}=t_{H}$, todas las características dejan de ser rectas, por lo que la solución analítica del problema resulta más compleja. En esta región el fluido se decelera hasta alcanzar la condición de contorno $u=0$ impuesta en la pared final de la cámara. De esta condición y de las Ecuaciones (3.1) y (3.2), sabiendo que 

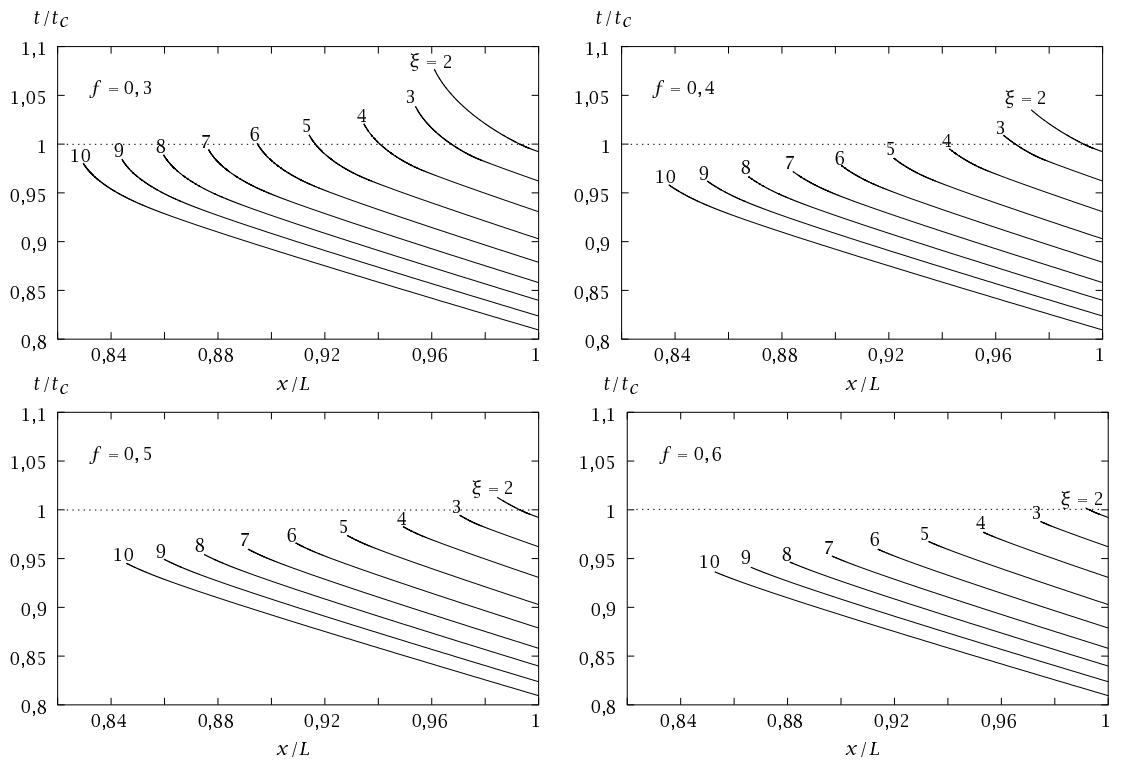

Figura 3.2: Curva $B C$ correspondiente a las Ecuaciones (3.10) y (3.10) para diferentes fracciones iniciales de llenado, $f$, y valores del parámetro $\xi$ satisfaciendo la condición $x_{c}=L$ según el modelo analítico sin reflexión. 
$u+2 c=2 X^{\prime}\left(t_{1}\right)+2 c_{0}$ a lo largo de las características positivas de esta región, se deduce que la pendiente de las características en la pared final de la cámara de inyección es:

$$
\left.\frac{\mathrm{d} t}{\mathrm{~d} x}\right|_{ \pm}= \pm \frac{1}{X^{\prime}\left(t_{1}\right)+c_{0}} .
$$

Para obtener las propiedades del flujo en esta región se usará una aproximación numérica del método de las características. Más adelante se detallará el procedimiento numérico empleado y se expondrán sus resultados más destacados.

Región de flujo uniforme (ECDI). Una vez que el pistón alcanza la velocidad crítica, $u_{H}$, deja de ser acelerado y mantiene su velocidad constante. De la condición de contorno $u=u_{H}$ impuesta en la superficie del pistón para $t_{1} \geq t_{H}$, y teniendo en cuenta la condición de la Ecuación (3.1) a lo largo de las características positivas que parten del pistón y de las características negativas que llegan a él, en las que se debe cumplir que $u-2 c=-2 c_{0}$, se deduce que la región $E C D I$ es una región de flujo uniforme en la que

$$
\begin{gathered}
c=c_{0} f^{-1 / 2}, \\
u=2 c_{0}\left(f^{-1 / 2}-1\right) .
\end{gathered}
$$

De la Ecuación (3.12) se deduce que la profundidad del metal fundido en esta región es igual a la altura máxima $H$ de la cámara de inyección.

Región de ondas simples (CFGD). Adyacente a la región ECDI existe otra región de ondas simples en la que el fluido tiene que decelerarse hasta alcanzar la condición de contorno, $u=0$, impuesta en la pared final de la cámara. En esta nueva región de ondas simples, la pendiente de las características positivas que partieron de la superficie del pistón aumenta conforme son alcanzadas por las características reflejadas en la pared final de la cámara. La pendiente de las características reflejadas disminuye desde el punto $C$, donde su valor se puede expresar a partir de las Ecuaciones (3.2), (3.12) y (3.13):

$$
\left.\frac{\mathrm{d} t}{\mathrm{~d} x}\right|_{-}=\frac{1}{c_{0}\left(f^{-1 / 2}-2\right)}
$$

hasta el punto $F$, donde su valor se puede obtener de la condición $u+2 c=$ $2 c_{0}\left(2 f^{-1 / 2}-1\right)$ que debe cumplir la característica positiva que llega a dicho punto y de la condición $u=0$ impuesta en la pared final de la cámara:

$$
\left.\frac{\mathrm{d} t}{\mathrm{~d} x}\right|_{+}=\frac{1}{c_{0}\left(2 f^{-1 / 2}-1\right)} \text {. }
$$


En esta región, $c$ aumenta desde $c_{0} f^{-1 / 2}$ a lo largo de la característica reflejada que pasa por los puntos $C$ y $D$ hasta $c_{0}\left(2 f^{-1 / 2}-1\right)$ a lo largo de la característica reflejada que pasa por los puntos $F$ y $G$. Esto implica que la profundidad del fluido aumentará por encima de la altura total $H$ de la cámara de inyección hasta alcanzar la profundidad máxima $H f\left(2 f^{-1 / 2}\right.$ $1)^{2}$. Obviamente, el techo de la cámara de inyección impedirá que la ola de metal fundido alcance una altura superior a $H$, por lo que ésta reflejará en la parte superior de la cámara. En el Capítulo 4 se estudiará en detalle la interacción de la ola con el techo de la cámara de inyección. Por otro lado, la característica originada en $t_{1}=0$ podría reflejarse contra la superficie del pistón provocando la aparición de otra región de ondas no simples; sin embargo, este caso no se estudiará aquí por tratarse de una situación de escaso interés práctico en el problema considerado.

Adyacente a esta región de ondas simples existe una región de flujo uniforme en la que se debe cumplir la condición $u \pm 2 c= \pm 2 c_{0}\left(2 f^{-1 / 2}-1\right)$, de donde se deduce que las propiedades del flujo son

$$
\begin{gathered}
c=c_{0}\left(2 f^{-1 / 2}-1\right), \\
u=0,
\end{gathered}
$$

correspondiente a fluido en reposo.

En la siguiente sección se detalla el procedimiento empleado para resolver el problema mediante una aproximación numérica del método de las características.

\subsection{Resolución numérica del modelo}

\subsubsection{Procedimiento numérico}

Para resolver numéricamente el sistema de Ecuaciones (3.1) y (3.2) se ha utilizado un esquema de diferencias finitas con una malla en el plano $x t$ en la que la localización de los puntos solución se especifica a priori ("Inverse Marching Method"). Las líneas características que pasan por cada punto solución se extienden hasta que intersectan con la línea de tiempo constante que contiene a los puntos solución calculados previamente. Las líneas características entre el punto solución y los puntos de intersección (en adelante se llamarán puntos iniciales) se aproximarán en cualquier caso por líneas rectas. Las ecuaciones en diferencias finitas resultantes de la discretización de las Ecuaciones (3.1) y (3.2), que más adelante serán expuestas, se resolverán usando el método predictorcorrector de Euler modificado. En el paso del predictor, los coeficientes de las ecuaciones en diferencias finitas se calcularán en los puntos iniciales, cuyas propiedades fluidas se determinarán a partir de una interpola- 
ción natural cúbica spline basada en los puntos solución previos ${ }^{\dagger}$. En el paso del corrector, los coeficientes se calculan para los valores promedio de las variables dependientes a lo largo de cada curva característica.

La malla de diferencias finitas que se ha empleado para resolver el problema se puede expresar como sigue:

$$
\begin{array}{cl}
x_{i}=X\left(t_{j}\right)+i \Delta x, & i=0,1, \ldots, I \\
t_{j}=t_{0}+j \Delta t, & j=0,1, \ldots, J
\end{array}
$$

donde $X\left(t_{j}\right)$ representa la coordenada $x$ de la superficie del pistón en el instante $t_{j}, I$ es el número de intervalos de la malla en la dirección $x$ y $J$ es el número de intervalos de tiempo. En adelante se escribirá $u_{i}^{j}$ y $c_{i}^{j}$ para representar las propiedades del flujo $u\left(x_{i}, t_{j}\right)$ y $c\left(x_{i}, t_{j}\right)$, respectivamente. En cada instante el paso $\Delta x$ se calcula como ${ }^{\ddagger}$

$$
\Delta x=\frac{L-X\left(t_{j}\right)}{I}
$$

y el paso $\Delta t$ se elige para asegurar la estabilidad del procedimiento numérico empleado, teniendo en cuenta el criterio de estabilidad de Courant, Friedrichs y Lewy (1928), tomándose

$$
\Delta t=\frac{\Delta x}{1,5(u+c)_{\max }}
$$

siendo $(u+c)_{\max }$ el valor máximo de $u+c$ en cada instante.

Las ecuaciones discretizadas correspondientes a las líneas características de la Ecuación (3.2) se pueden expresar como

$$
\Delta t=\lambda_{ \pm} \Delta x
$$

donde $\lambda_{ \pm}$corresponde a la pendiente de las características,

$$
\lambda_{ \pm}=\frac{1}{u \pm c} .
$$

El código del procedimiento numérico empleado se ha dividido en 3 subrutinas llamadas PISTÓN, INTER y PARED, que resuelven el problema en puntos situados en la superficie del pistón, en el interior de la cámara de inyección y en la pared final, respectivamente. En las Figuras 3.3, 3.4 y 3.5 se representan los diagramas de flujo correspondientes a las tres subrutinas anteriores. A continuación se empezará detallando el procedimiento utilizado para un punto situado en el interior de la cámara de inyección.

\footnotetext{
${ }^{\dagger}$ El procedimiento empleado para interpolar las propiedades del flujo en los puntos iniciales se puede ver en detalle en la sección 5.3.3.

${ }^{\ddagger}$ En cada paso de tiempo, los puntos solución previos se desplazan. Las propiedades del flujo en los puntos desplazados se obtendrán usando el mismo tipo de interpolación que en los puntos iniciales.
} 


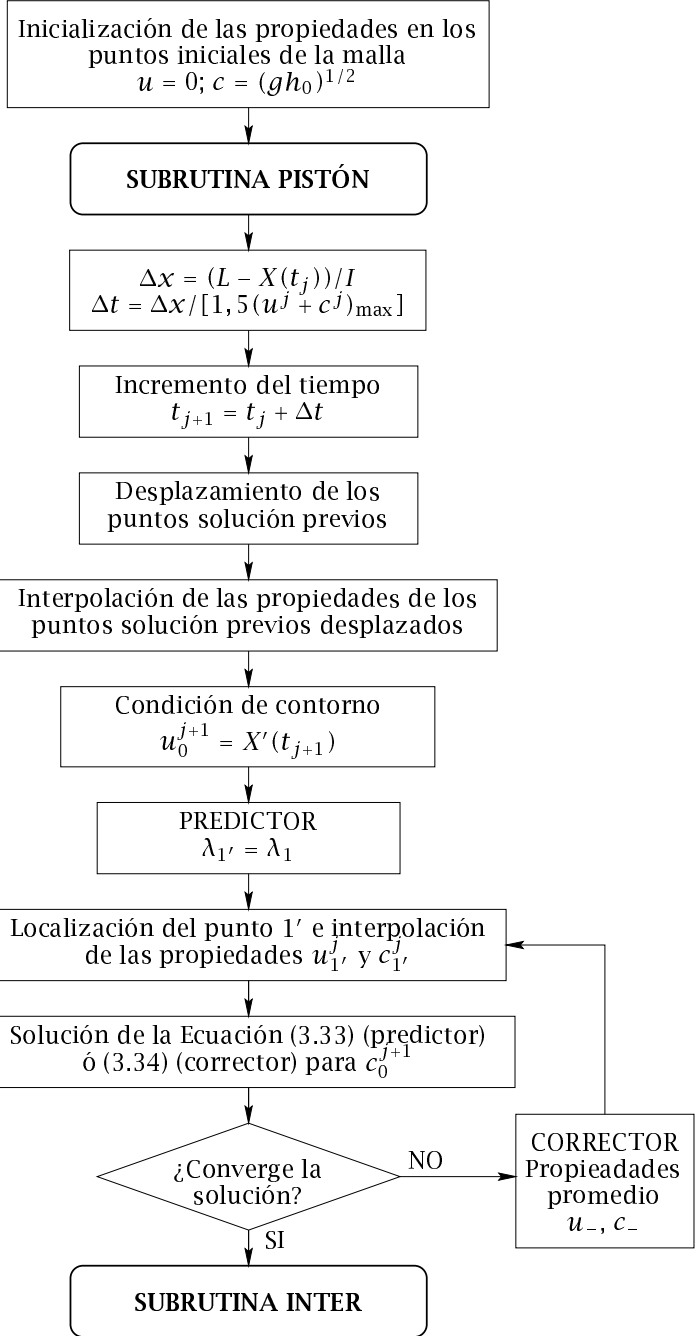

Figura 3.3: Diagrama de flujo de la subrutina PISTÓN para calcular las propiedades del flujo en un punto situado en la superficie del pistón. 


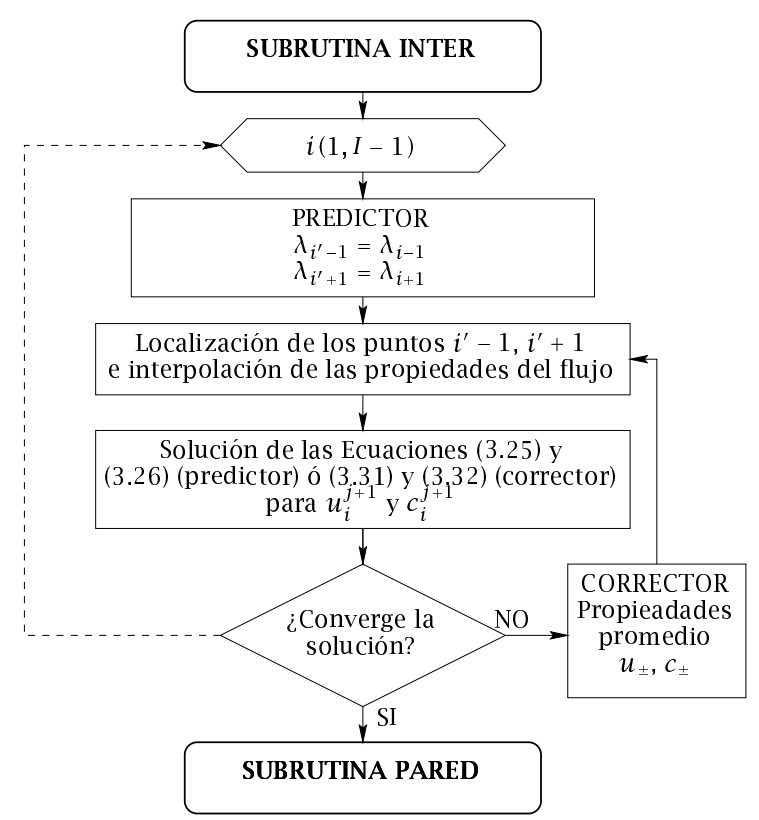

Figura 3.4: Diagrama de flujo de la subrutina INTER para calcular las propiedades del flujo en un punto situado en el interior de la cámara de inyección. 


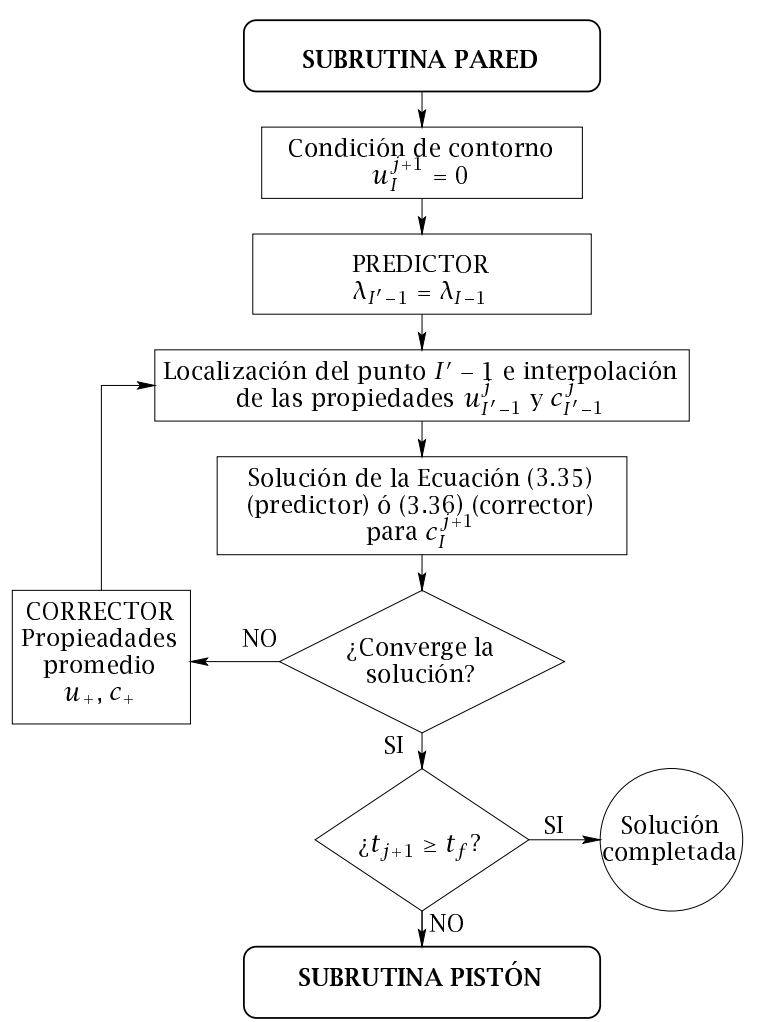

Figura 3.5: Diagrama de flujo de la subrutina PARED para calcular las propiedades del flujo en un punto situado en la pared final de la cámara de inyección. 


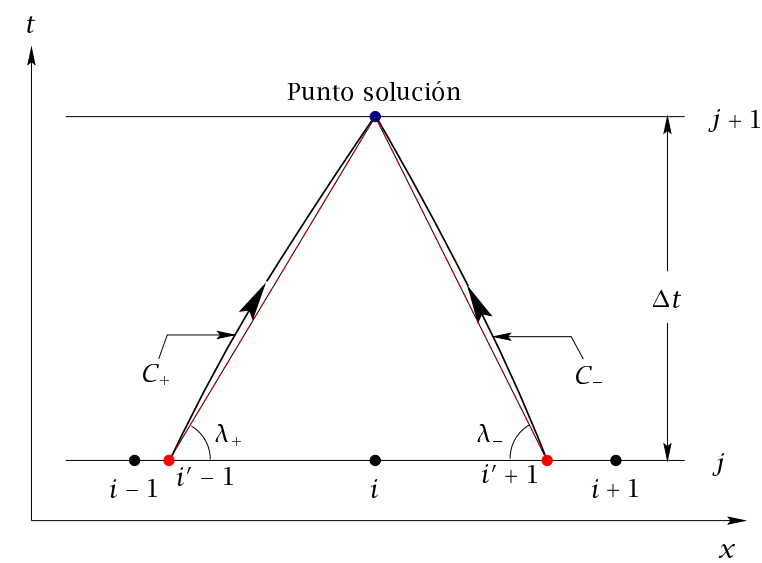

Figura 3.6: Malla de diferencias finitas en el plano $x t$ para un punto situado en el interior de la cámara de inyección.

\subsubsection{Interior de la cámara de inyección}

Predictor.- En la Figura 3.6 se representa la malla de diferencias finitas correspondiente a un punto $i$ que se encuentra en el interior de la cámara de inyección, donde $i=1, \ldots, I-1$. $i=0$ e $i=I$ corresponden a los puntos situados en la superficie del pistón y la pared final de la cámara de inyección, respectivamente. Las ecuaciones características discretizadas se pueden expresar para este tipo de malla del siguiente modo:

$$
\begin{aligned}
& \Delta t=\lambda_{+}\left(x_{i}^{j+1}-x_{i^{\prime}-1}^{j}\right), \\
& \Delta t=\lambda_{-}\left(x_{i}^{j+1}-x_{i^{\prime}+1}^{j}\right),
\end{aligned}
$$

y las correspondientes ecuaciones de compatibilidad se pueden expresar como:

$$
\begin{aligned}
& u_{i}^{j+1}+2 c_{i}^{j+1}=u_{i^{\prime}-1}^{j}+2 c_{i^{\prime}-1}^{j}, \\
& u_{i}^{j+1}-2 c_{i}^{j+1}=u_{i^{\prime}+1}^{j}-2 c_{i^{\prime}+1}^{j},
\end{aligned}
$$

donde el subíndice $i^{\prime}$ se refiere a los puntos iniciales en el instante $t_{j}$ por los que pasan las características que llegan al punto solución y cuyas propiedades se obtienen por interpolación a partir de los valores de las propiedades del flujo en los puntos solución previos. Para iniciar el predictor se supondrá que los puntos iniciales coinciden con los puntos solución previos, es decir, $x_{i^{\prime}-1}^{j}=x_{i-1}^{j}$ y $x_{i^{\prime}+1}^{j}=x_{i+1}^{j}$. De esta forma, las 
pendientes de las líneas características podrán ser estimadas a partir de la expresión:

$$
\lambda_{+}^{P}=\lambda_{i^{\prime}-1}^{j}=\frac{1}{u_{i-1}^{j}+c_{i-1}^{j}} ; \lambda_{-}^{P}=\lambda_{i^{\prime}+1}^{j}=\frac{1}{u_{i+1}^{j}-c_{i+1}^{j}},
$$

donde el superíndice $P$ denota el valor correspondiente al predictor. La localización de los nuevos puntos iniciales $\left(i^{\prime}-1, j\right)$ e $\left(i^{\prime}+1, j\right)$ se determina prolongando las curvas características, consideradas líneas rectas, desde el punto solución $(i, j+1)$ hasta cortar a la línea de tiempo constante $t_{j}$. De esta forma, y a partir de las Ecuaciones (3.23) y (3.24), se obtiene, respectivamente

$$
\begin{aligned}
& x_{i^{\prime}-1}^{j}=x_{i}^{j+1}-\frac{\Delta t}{\lambda_{+}^{P}}, \\
& x_{i^{\prime}+1}^{j}=x_{i}^{j+1}-\frac{\Delta t}{\lambda_{-}^{P}} .
\end{aligned}
$$

Los valores de las propiedades del flujo correspondientes a los puntos iniciales se obtendrán a continuación por interpolación a partir de los valores solución previos. Una vez determinada la localización de los puntos iniciales y las propiedades del flujo en ellos, la pendiente de las curvas características podrá ser de nuevo evaluada en dichos puntos y repetir los cálculos anteriores. Este procedimiento se repetirá iterativamente para mejorar la precisión de los valores de las posiciones en los puntos iniciales y las propiedades del flujo en ellos.

Conocidas las propiedades del flujo en los puntos iniciales $\left(x_{i^{\prime}-1}^{j} \mathrm{y}\right.$ $x_{i^{\prime}+1}^{j}$ ), se completará la aplicación del predictor resolviendo el sistema de Ecuaciones (3.25) y (3.26) para obtener las propiedades del flujo $u_{i}^{j+1}$ y $c_{i}^{j+1}$ en el punto solución.

Corrector.- Para iniciar el corrector, las propiedades del flujo a lo largo de las características que llegan al punto solución se supondrán uniformes e iguales a los valores promedio entre las propiedades del flujo en los puntos iniciales y las del punto solución obtenidas en la aplicación del predictor:

$$
\begin{gathered}
u_{+}=\frac{u_{i^{\prime}-1}^{j}+u_{i}^{j+1} P}{2} ; u_{-}=\frac{u_{i^{\prime}+1}^{j}+u_{i}^{j+1} P}{2}, \\
c_{+}=\frac{c_{i^{\prime}-1}^{j}+c_{i}^{j+1} P}{2} ; c_{-}=\frac{c_{i^{\prime}+1}^{j}+c_{i}^{j+1} P}{2} .
\end{gathered}
$$

Con estos valores se calcularán las pendientes de las líneas características y se corregirán las posiciones de los puntos iniciales $\left(i^{\prime}-1, j\right)$ e $\left(i^{\prime}+1, j\right)$ 


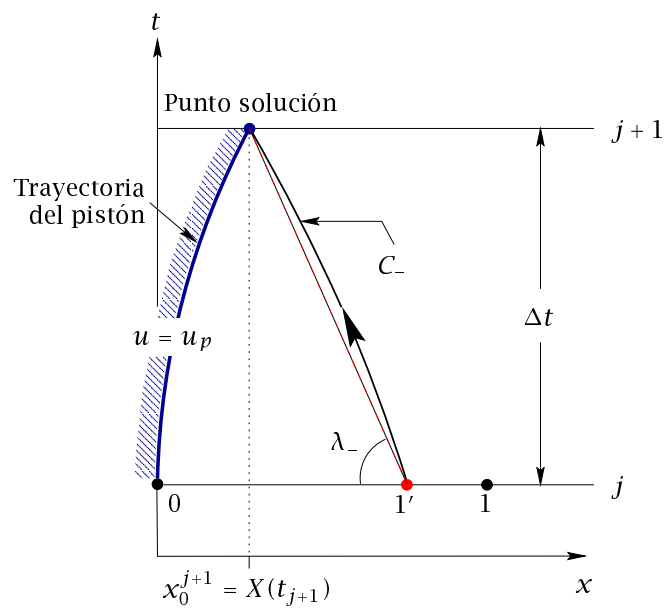

Figura 3.7: Malla de diferencias finitas en el plano $x t$ para un punto situado en la superficie del pistón.

empleando el mismo procedimiento descrito en la aplicación del predictor. Corregidas las posiciones y las propiedades del flujo en los puntos iniciales, y obtenidos los valores promedio de la Ecuación (3.30), las propiedades corregidas del punto solución podrán ser obtenidas resolviendo el sistema de ecuaciones

$$
\begin{aligned}
& u_{i}^{j+1}+2 c_{i}^{j+1}=u_{+}+2 c_{+}, \\
& u_{i}^{j+1}-2 c_{i}^{j+1}=u_{-}-2 c_{-} .
\end{aligned}
$$

El corrector se aplicará iterativamente para mejorar la precisión del valor de la solución final. Se ha comprobado que, generalmente, aplicando una o dos veces el corrector, los resultados obtenidos son suficientemente precisos.

\subsubsection{Superficie del pistón}

En la Figura 3.7 se representa la malla utilizada para calcular las propiedades del flujo en la superficie del pistón. La posición del punto solución $X\left(t_{j+1}\right)$ y la velocidad del flujo $X^{\prime}\left(t_{j+1}\right)$ en dicho punto están determinadas por la ley de movimiento del pistón. Para completar la solución del problema en este punto, se empleará básicamente el mismo procedimiento descrito para el caso de un punto situado en el interior de la cámara de inyección. En el predictor, a partir de la ecuación de compatibilidad 


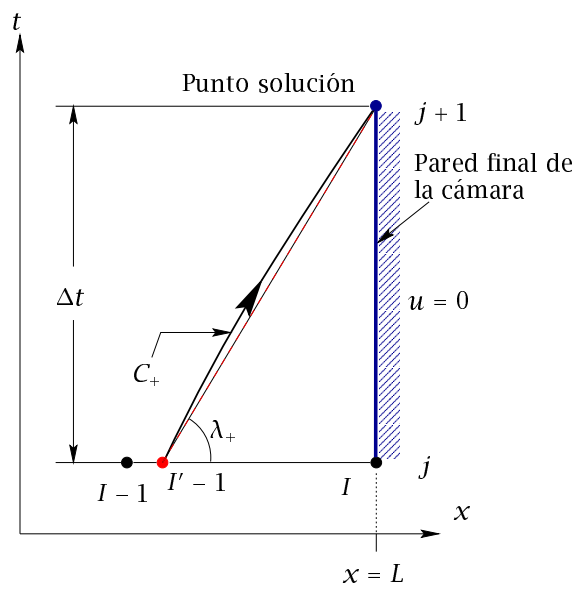

Figura 3.8: Malla de diferencias finitas en el plano $x t$ para un punto situado en la pared final de la cámara de inyección.

válida a lo largo de la característica negativa que llega al punto solución, se obtiene

$$
c_{0}^{j+1} P=\frac{X^{\prime}\left(t_{j+1}\right)+\left(2 c_{1^{\prime}}^{j}-u_{1^{\prime}}^{j}\right)}{2},
$$

y a partir de las propiedades promedio entre los puntos $\left(1^{\prime}, j\right)$ y $(0, j+1)$ para la característica negativa, el $\underline{\text { corrector resulta }}$

$$
c_{0}^{j+1}{ }^{C}=\frac{X^{\prime}\left(t_{j+1}\right)+\left(2 c_{-}-u_{-}\right)}{2} .
$$

\subsubsection{Pared final de la cámara de inyección}

En la pared final de la cámara de inyección, la velocidad del flujo en todo instante es $u=0$. Para completar la solución en el punto situado en la pared final de la cámara se resolverá la ecuación de compatibilidad válida a lo largo de la característica positiva que llega a dicho punto. En la Figura 3.8 se representa la malla de diferencias finitas para obtener las propiedades del flujo en este caso. Siguiendo el mismo procedimiento que en los casos anteriores, en el predictor

$$
c_{I}^{j+1} P=\frac{u_{I^{\prime}-1}^{j}+2 c_{I^{\prime}-1}^{j}}{2}
$$


y a partir de las propiedades promedio entre los puntos $\left(I^{\prime}-1, j\right)$ e $(I, j+1)$ para la característica positiva, el corrector resulta

$$
c_{I}^{j+1}{ }^{C}=\frac{u_{+}+2 c_{+}}{2} .
$$

\subsubsection{Precisión del modelo numérico}

Para comprobar la precisión del modelo numérico, sus resultados se han comparado con los obtenidos a partir de una solución analítica correspondiente a la ley de movimiento de la Ecuación (2.44) con $n=1$. Introduciendo las Ecuaciones (2.42) y (2.44) en la Ecuación (2.8), y a partir de las Ecuaciones (2.9) y (2.10), se obtiene

$$
\begin{gathered}
u_{\text {exact }}=-\frac{1}{2}\left(c_{0}-\frac{3}{2} \gamma t\right)+\frac{1}{2}\left[\left(c_{0}-\frac{3}{2} \gamma t\right)^{2}-4 \gamma\left(x-c_{0} t\right)\right]^{1 / 2}, \\
c_{\text {exact }}=c_{0}-\frac{1}{4}\left(c_{0}-\frac{3}{2} \gamma t\right)+\frac{1}{4}\left[\left(c_{0}-\frac{3}{2} \gamma t\right)^{2}-4 \gamma\left(x-c_{0} t\right)\right]^{1 / 2} .
\end{gathered}
$$

La precisión de segundo orden del esquema numérico empleado se puede apreciar en la Figura 3.9, en la que se representa el error relativo definido por

$$
E=\left(u_{\text {exact }}-u_{\Delta x}\right) / u_{\text {exact }},
$$

donde $u_{\Delta x}$ y $u_{\text {exact }}$ son, respectivamente, la solución numérica aproximada y la correspondiente solución exacta de la Ecuación (3.37) en $t=L / c_{0}$, en función del tamaño adimensional de la malla $\Delta x / L$, para un caso con $f=0,5$ y $\gamma=32,7$. La malla más fina que se ha utilizado en este análisis corresponde a $\Delta x / L=6,3 \times 10^{-4}$. La curva representada en la Figura 3.9 corresponde a la sección intermedia entre la superficie del pistón y la pared final de la cámara de inyección en el instante considerado. Todos los resultados de esta figura se han obtenido utilizando un valor igual a 5,05 para la relación $\Delta x /\left(c_{0} \Delta t\right)$ al principio del movimiento del pistón.

En otros casos, para los que no se dispone de resultados analíticos y en los que los efectos de reflexión de la ola contra la pared final de la cámara son importantes, se ha usado el método de extrapolación de Richardson (Celik y Zhang, 1995) para comprobar la precisión del modelo numérico que se ha empleado. Como ejemplo, en la Figura 3.10 se ha representado, para la ley de movimiento del pistón de la Ecuación (2.17) con $\xi=3, L / \beta=9,46$, fracción inicial de llenado $f=0,25$ y un instante $t=1,08 c_{0} / L$ para el que la ola ya se ha reflejado contra la pared final de la cámara, el error numérico aproximado, relativo al refinamiento de la malla, definido por

$$
E=\left(c_{\mathrm{ext}}-c_{\Delta x}\right) / c_{\mathrm{ext}},
$$




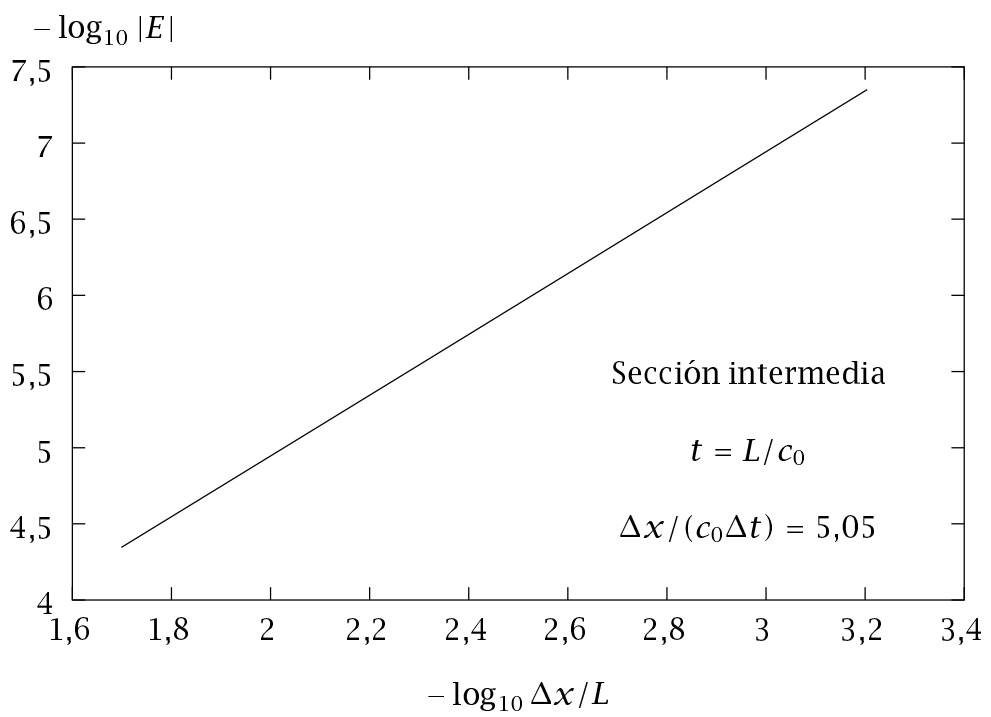

Figura 3.9: Error numérico de la Ecuación (3.39) en función del tamaño adimensional de la malla $\Delta x / L$, en la sección intermedia entre la superficie del pistón y la pared final de la cámara, y $t=L / c_{0}(f=0,5$ y ley de movimiento del pistón de la Ecuación (2.42) con $\gamma=32,7)$. 
donde $c_{\Delta x}$ es la solución numérica aproximada y $c_{\text {ext }}$ es la solución numérica extrapolada, que se ha calculado como

$$
c_{\mathrm{ext}}=\frac{2^{a} c_{\Delta x}-c_{2 \Delta x}}{2^{a}-1}
$$

siendo $a$ el orden aparente del método numérico, que puede ser calculado mediante la expresión

$$
a=\frac{\ln \left[\left(c_{2 \Delta x}-c_{4 \Delta x}\right)\left(c_{\Delta x}-c_{2 \Delta x}\right)\right]}{\ln 2} .
$$

La malla más fina que se ha utilizado en este análisis corresponde a

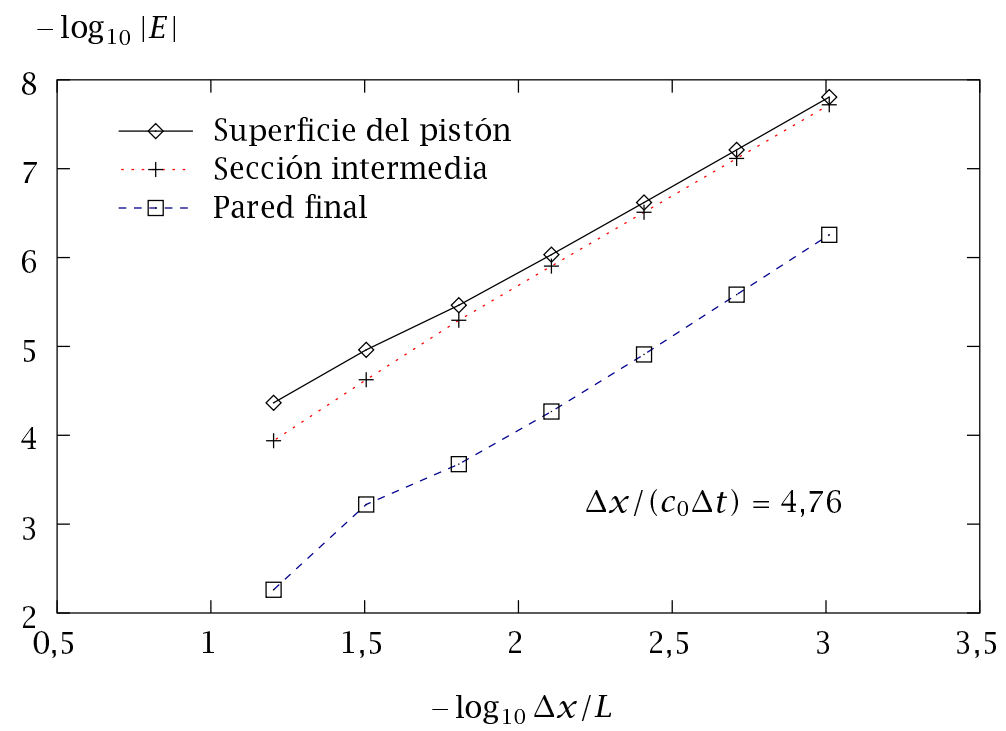

Figura 3.10: Error relativo al refinamiento de la malla definido por la Ecuación (3.40) en función del tamaño adimensional de la malla $\Delta x / L$, en diferentes secciones de la cámara $\mathrm{y}$ $t c_{0} / L=1,08$ (ley de aceleración del pistón de la Ecuación (2.16), $f=0,25, \xi=3$ y $L / \beta=9,46$.

$\Delta x / L=10^{-3}$. Las tres curvas representadas en la Figura 3.10 corresponden a la superficie del pistón, la pared final de la cámara de inyección y la sección intermedia entre ambas. Los valores del orden del método calculados en estas tres posiciones son 1,97, 2,24 y 2,01, respectivamente. Todos los resultados mostrados en la Figura 3.10 se han obtenido con un valor de 4,76 para $\Delta x /\left(c_{0} \Delta t\right)$ al principio del movimiento del pistón.

Aunque se podría haber realizado una comparación más extensa con otras soluciones analíticas, en el Capítulo 5 se analizará con más detalle la 
precisión de un esquema numérico, similar al presentado en este capítulo, para estudiar el flujo compresible unidimensional no estacionario de un gas perfecto.

\subsection{Discusión de resultados}

\subsubsection{Perfiles de la superficie libre de metal fundido}

En la Figura 3.11 se representan los perfiles de la ola en el instante en el que se forma una discontinuidad (perfil de ola vertical) con la ley de aceleración del pistón de la Ecuación (2.16), para diferentes valores del parámetro $\xi$ y distintas fracciones iniciales de llenado. Los parámetros de la ley de aceleración se han elegido para satisfacer la condición $x_{c}=$ $0,975 L, \$$ lo que permitirá apreciar la influencia de la reflexión del metal fundido sobre la rotura de la superficie de la ola. En esta figura también se ha representado, con líneas de puntos, el perfil de la ola correspondiente al modelo sin reflexión en el instante $t=t_{c}=\left[\frac{4}{3}+\ln \left(\xi-\frac{1}{3}\right)\right] / \alpha$ para $\xi=5$. Aunque la mayoría de las condiciones representadas en la Figura 3.11 están alejadas de las óptimas, se puede observar que la reflexión de ola en la pared final de la cámara tiende a hacer que la rotura se empiece a producir un poco antes de la posición que se predice cuando se desprecian los efectos de reflexión $\left(x_{c}=0,975 L\right)$. El adelanto de la rotura de la ola se hace más importante conforme aumenta el parámetro $\xi$.

En las Figuras 3.12a, 3.12b, 3.13a y 3.13b se comparan, para fracciones iniciales de llenado de 0,25, 0,3, 0,4 y 0,5, respectivamente, los resultados de los perfiles que cumplen la condición de que la ola reflejada empiece a romperse en el instante en el que el metal fundido alcanza la entrada hacia la cavidad del molde (situada en $x=L, h=H$ ) con los obtenidos despreciando los efectos de reflexión. Para cada valor de $f$, los perfiles representados corresponden a diferentes combinaciones de los parámetros adimensionales $\xi$ y $L / \beta$ que satisfacen la condición que se acaba de mencionar. Para cada uno de los casos representados en estas figuras, el instante que satisface esta condición es el correspondiente al valor de $t / t_{f}$ del último perfil representado, donde $t_{f}$ es el tiempo de llenado de la cámara de inyección. En estas figuras se puede observar que, para este instante, el área ocupada por el aire en la cámara de inyección disminuye conforme aumenta el parámetro $\xi$, lo que está de acuerdo con los resultados del modelo más simple representados en la Figura 2.7 ^. Se

\footnotetext{
${ }^{\S}$ Esta condición establece la coordenada $x$ donde, según el modelo analítico sin reflexión del Capítulo 2, se formará la discontinuidad.

"Aunque los resultados de la Figura 2.7, correspondientes al modelo analítico sin reflexión, se han obtenido satisfaciendo una condición distinta a la impuesta en las Figuras
} 

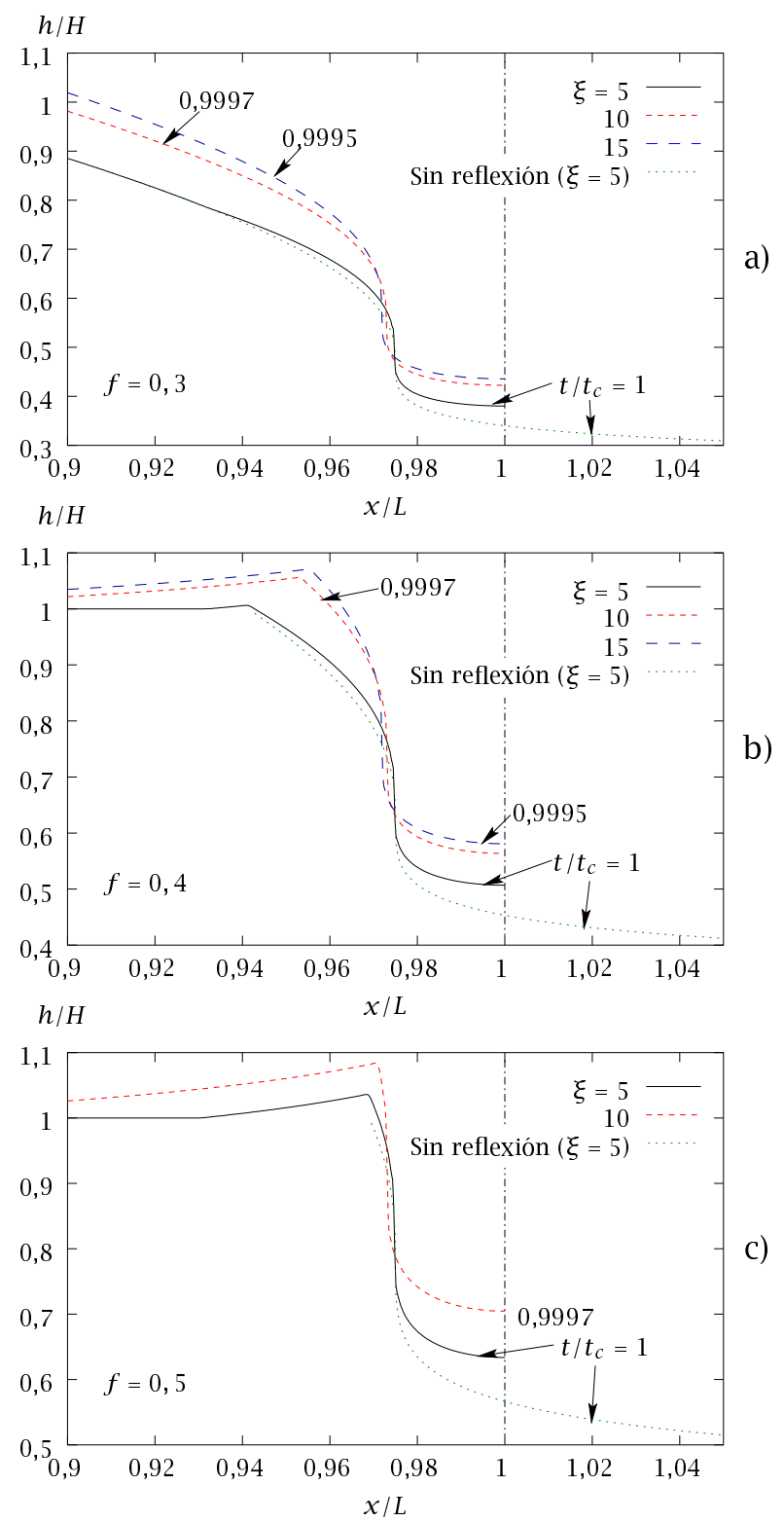

Figura 3.11: Perfiles de la ola en el instante en el que se forma una discontinuidad para la ley de aceleración del pistón de la Ecuación (2.16), con diferentes valores de $\xi$ y distintas fracciones iniciales de llenado, que satisfacen la condición $x_{c}=0,975 L$, según el modelo analítico sin reflexión. 

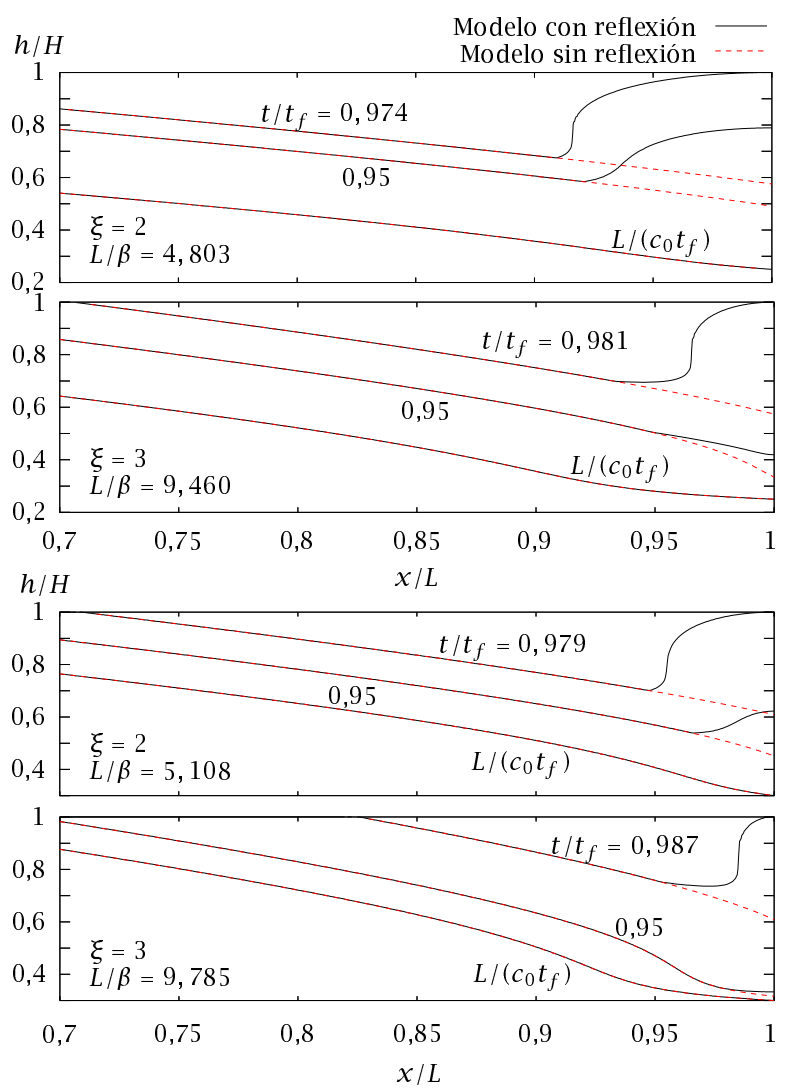

(b)

Figura 3.12: Comparación entre los resultados de los perfiles para condiciones en las que la ola reflejada empieza a romperse en el instante en el que el metal fundido alcanza la entrada hacia la cavidad del molde y los obtenidos despreciando los efectos de reflexión. a) $f=0,25$, b) $f=0,3$. 


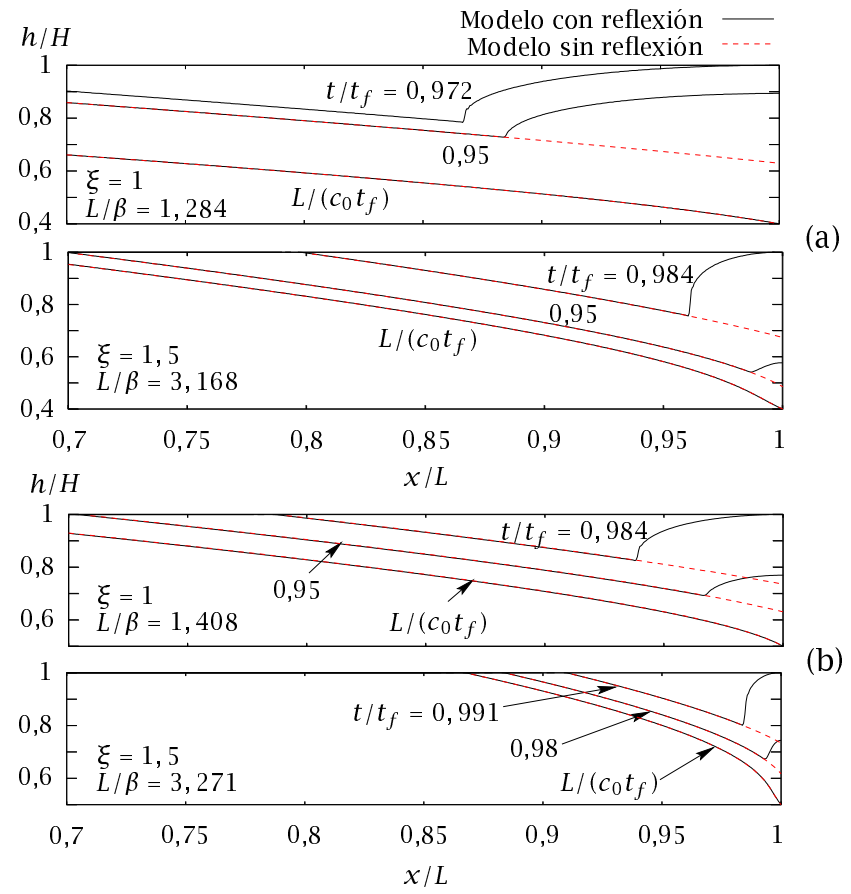

Figura 3.13: Comparación entre los resultados de los perfiles para condiciones en las que la ola reflejada empieza a romperse en el instante en el que el metal fundido alcanza la entrada hacia la cavidad del molde y los obtenidos despreciando los efectos de reflexión. a) $f=0,4$, b) $f=0,5$. 
puede observar que, como cabía esperar, para los instantes representados en los que aún no se ha iniciado la reflexión de la ola", la concordancia entre el modelo analítico sin reflexión y el modelo numérico con reflexión es excelente. Es importante recordar que no sólo se trata de reducir el aire atrapado, sino también de reducir tanto como sea posible el tiempo de llenado para aumentar la productividad del proceso y evitar solidificaciones prematuras del metal fundido durante el proceso de inyección. Por lo tanto, las condiciones óptimas de operación serán el resultado del doble compromiso de reducción del aire atrapado y del tiempo de llenado. Para facilitar la selección de condiciones adecuadas de operación, en la siguiente sección se lleva a cabo un estudio de la influencia de los parámetros de la ley de aceleración en el volumen de aire atrapado y el tiempo de llenado para distintas fracciones iniciales de llenado.

\subsubsection{Volumen de aire atrapado}

En las Figuras 3.14 y 3.15, que corresponden a las fracciones iniciales de llenado de 0,25 y 0,4 , respectivamente, se representan los resultados obtenidos para el área adimensional ocupada por el aire en la cámara de inyección cuando el metal fundido alcanza la entrada hacia la cavidad del molde, $A /(H L)$, en función de $L / \beta$, para diferentes valores de $\xi$. Se espera que la cantidad de aire finalmente atrapado en el metal fundido esté relacionada con este área. En la parte superior de estas figuras, se representa el tiempo de llenado adimensional $t_{f} c_{0} / L$. Se puede ver en las Figuras 3.14 y 3.15 que para un valor de $\xi$ dado, excepto para $f=0,25$ y $\xi=2$, existe un máximo local de aire atrapado para un valor de $L / \beta$ que aumenta con $\xi$ (obsérvese que, para valores de $\xi, L$ y $f$ dados, aumentar $L / \beta$ supone aumentar el parámetro $\alpha$ ). Este máximo valor de aire atrapado disminuye conforme aumenta $\xi$. En cada curva, para un valor dado de $\xi$, el círculo negro representa el valor $(L / \beta)_{\text {opt }}$ que hace que la ola que se refleja en la pared final de la cámara empiece a romperse justo en el instante en el que el metal fundido alcanza la entrada hacia la cavidad del molde, que es la misma condición satisfecha por los perfiles de ola representados en las Figuras 3.12 y 3.13. Obsérvese que este punto está situado a la derecha del máximo. Cuando $L / \beta$ aumenta por encima de $(L / \beta)_{\text {opt }}$, para un valor dado de $\xi$, la ola debería empezar a romperse antes, lo que en principio cabe esperar que incrementaría la ingestión de aire y, por tanto, la cantidad de aire atrapado. Por lo tanto, puede existir un mínimo local de aire atrapado alrededor de $(L / \beta)$ opt .

3.12 y 3.13 , se puede observar en ambos modelos la misma tendencia cualitativa del área $A /(H L)$ conforme el parámetro $\xi$ aumenta.

$\|$ El primer perfil de la ola que se ha representado en cada una de las Figuras 3.12a, $3.12 \mathrm{~b}, 3.13 \mathrm{a}$ y $3.13 \mathrm{~b}$, corresponde al instante $t / t_{f}=L /\left(c_{0} t_{f}\right)$ para el que el extremo más avanzado de la ola alcanza la pared final de la cámara de inyección. 


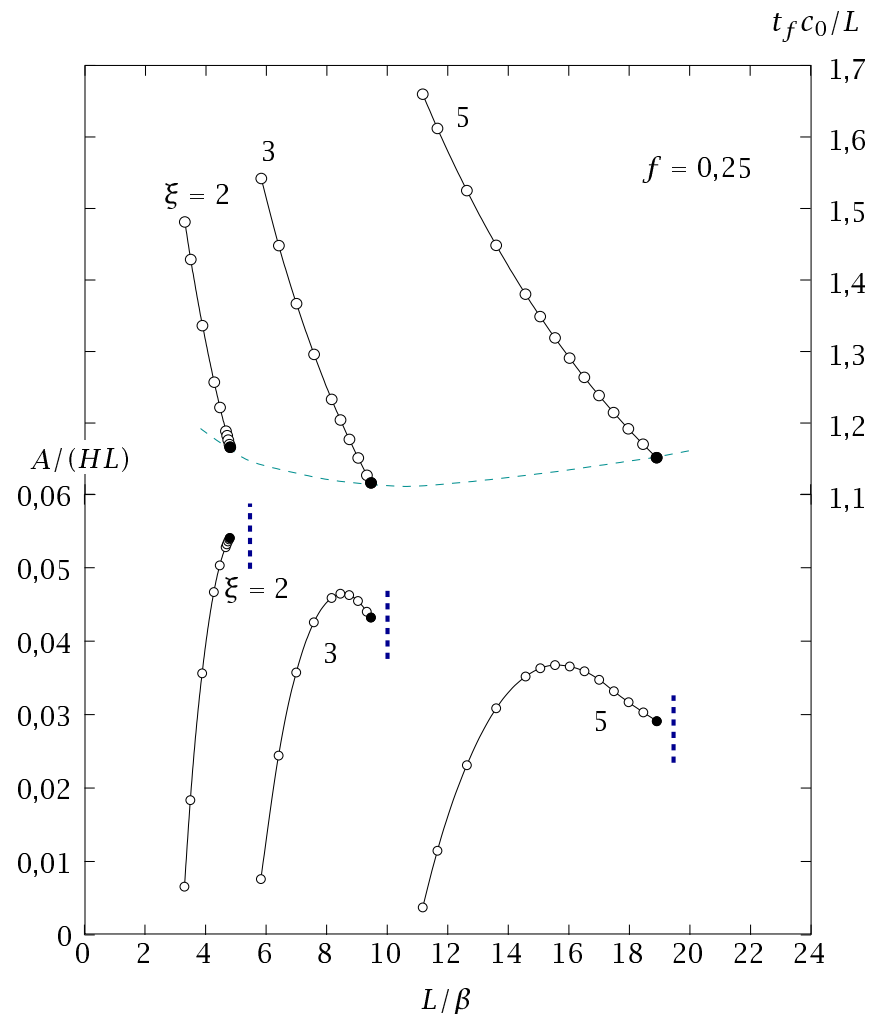

Figura 3.14: Resultados del área adimensional ocupada por el aire cuando el metal fundido alcanza la entrada hacia la cavidad del molde y del tiempo de llenado adimensional en función de $L / \beta$, para diferentes valores de $\xi$, y $f=0,25$. 


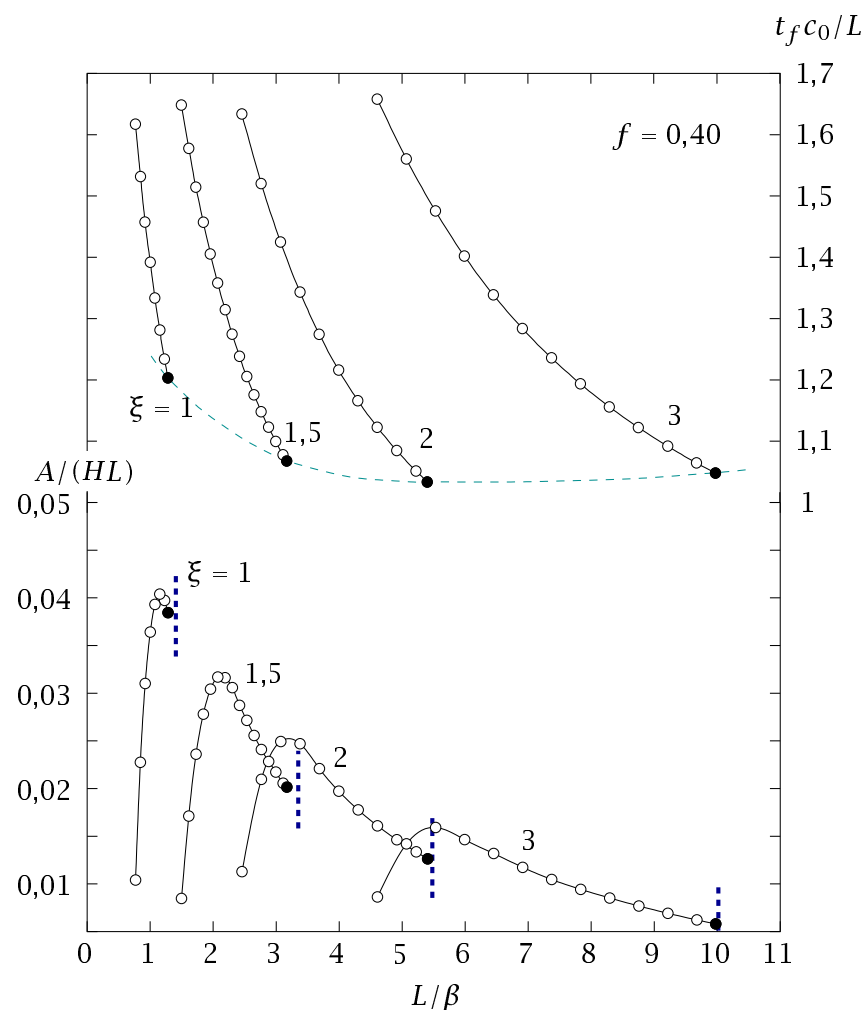

Figura 3.15: Resultados del área adimensional ocupada por el aire cuando el metal fundido alcanza la entrada hacia la cavidad del molde y del tiempo de llenado adimensional en función de $L / \beta$, para diferentes valores de $\xi$, y $f=0,4$. 
Además, el tiempo de llenado es también mínimo en $(L / \beta)_{\text {opt }}$ en el rango $L / \beta \leq(L / \beta)_{\text {opt }}$, por lo que, para un valor dado de $\xi,(L / \beta)_{\text {opt }}$ sería una elección correcta cuando conseguir un pequeño tiempo de llenado es un requerimiento esencial. Por otro lado, se debería destacar que eligiendo un valor suficientemente bajo de $L / \beta$ a la izquierda del máximo, para un $\xi$ dado, se podría obtener una cantidad más pequeña de aire atrapado, aunque los tiempos de llenado aumentarían considerablemente.

En las Figuras 3.14 y 3.15 también se representa, con una pequeña línea discontinua vertical, el valor equivalente al $(L / \beta)$ opt obtenido analíticamente mediante el modelo basado en la aproximación de aguas poco profundas pero despreciando los efectos de reflexión de la ola. En este modelo, el valor "óptimo" del parámetro $L / \beta$ para un valor dado de $\xi$ se consideró ser el que satisface la condición de que el frente de ola empiece a romperse exactamente en la pared final de la cámara de inyección. Se puede apreciar en las Figuras 3.14 y 3.15 que los valores de $L / \beta$ determinados de este modo para diferentes valores de $\xi$ están muy próximos a los obtenidos considerando los efectos de reflexión de la ola en la pared final de la cámara (representados con círculos negros). Sin embargo, el interés del modelo descrito en este capítulo está justificado por la necesidad de analizar en detalle las variaciones en el volumen de aire atrapado usando parámetros de aceleración alrededor del valor "óptimo", y esto sólo se puede hacer teniendo en cuenta los efectos de reflexión de la ola. En el capítulo siguiente se demostrará que, en realidad, los efectos no hidrostáticos tienden a suavizar la ola y retrasar el momento de la rotura, por lo que los valores óptimos de $L / \beta$ serán en principio mayores que los encontrados con el modelo semianalítico descrito en este capítulo.

\subsubsection{Elección de las condiciones de operación}

En la Figura 3.16 se representa el valor adimensional del área $A /(H L)$ y el tiempo de llenado $t_{f} \mathcal{C}_{0} / L$ en función de $\xi$ para $L / \beta=(L / \beta)_{\text {opt }}$ (es decir, para las condiciones de los puntos representados con círculos negros en las Figuras 3.14 y 3.15) y diferentes valores de la fracción inicial de llenado. La Figura 3.16, junto con las Figuras 3.14 y 3.15, pueden ser útiles para elegir valores orientativos adecuados de los parámetros de trabajo. En la Figura 3.16 se puede observar que el tiempo adimensional de llenado disminuye conforme $f$ aumenta para cualquier valor de $\xi$, y que su valor mínimo se obtiene para un valor de $\xi$ que aumenta conforme $f$ disminuye**. También se observa que el valor adimensional del área $A /(H L)$ disminuye rápidamente conforme $\xi$ aumenta para fracciones ini-

\footnotetext{
**Obsérvese que la tendencia del tiempo de llenado mínimo conforme aumentan el parámetro $\xi$ y la fracción inicial de llenado coincide con la tendencia de los resultados representados en la Figura 2.6, que se han obtenido para los parámetros óptimos del modelo analítico despreciando los efectos de reflexión.
} 


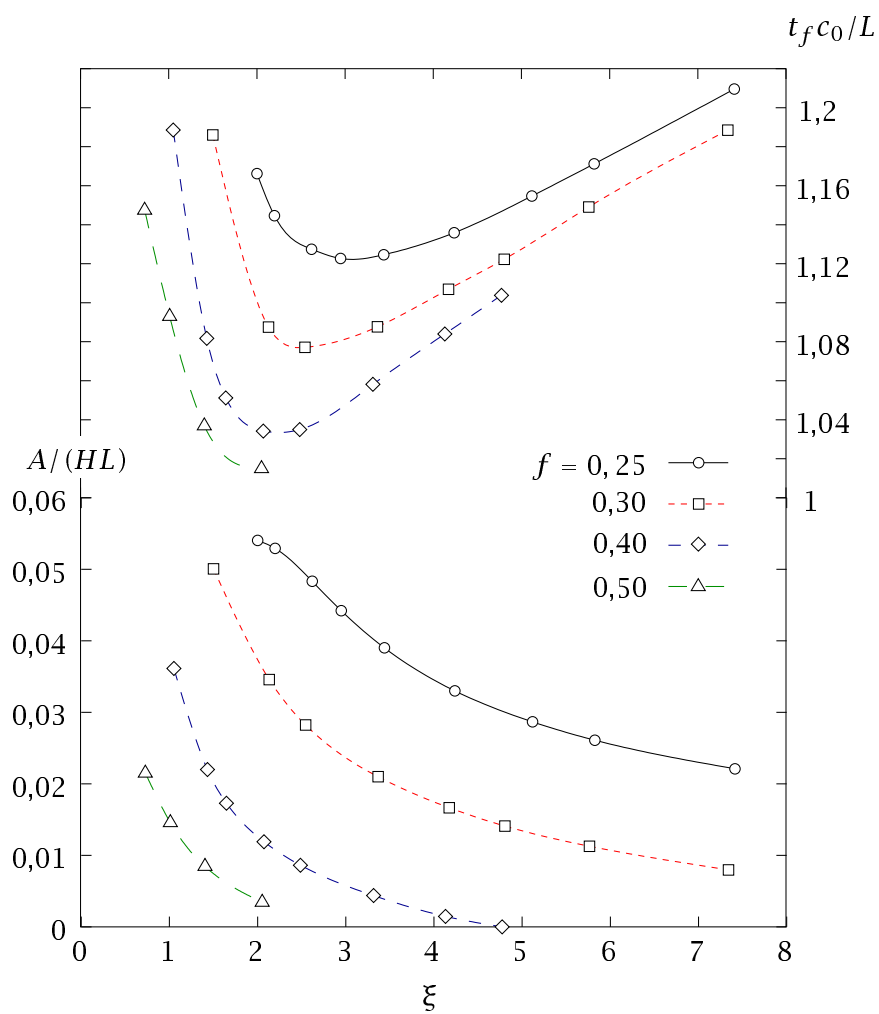

Figura 3.16: Resultados del valor del área adimensional $A /(H L)$ y del tiempo de llenado en función de $\xi$, para diferentes fracciones iniciales de llenado y $L / \beta=(L / \beta)_{\text {opt }}$. 
ciales de llenado más altas, y llega a ser relativamente bajo para valores de $\xi$ próximos al valor correspondiente al tiempo de llenado mínimo. Para valores de $f$ bajos, sin embargo, sólo se pueden obtener cantidades comparables de aire atrapado para valores más altos de $\xi$, para los que el tiempo de llenado será muy grande.

Para apreciar mejor la diferencia entre los resultados óptimos obtenidos en este capítulo y los correspondientes al modelo más simple en el que se desprecian los efectos de reflexión de la ola, en la Figura 3.17 se representa la relación $(L / \beta)_{\mathrm{opt}} /(L / \beta)_{\mathrm{opt}}$, siendo el parámetro $(L / \beta)_{\mathrm{opt}}$ el que satisface la condición de que el frente de la ola empiece a romperse exactamente en la pared final de la cámara de inyección, el cual se ha obtenido, para los casos representados en esta figura, de la Ecuación (2.33) para valores de $\xi>4 / 3$, y de la Ecuación (2.35) para valores de $\xi \leq 4 / 3$. Obsérvese que las diferencias en términos relativos entre ambos modelos

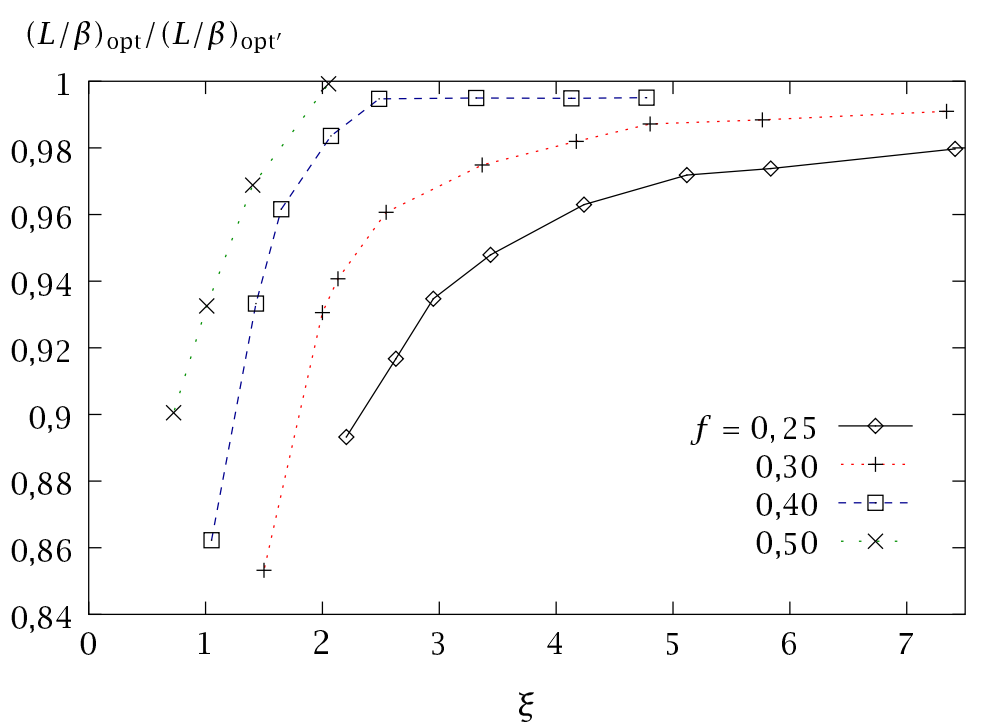

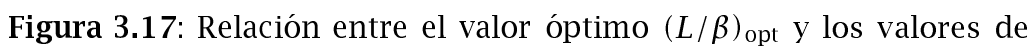
las Ecuaciones (2.33) y (2.35) correspondientes al modelo analítico sin reflexión, en función de $\xi$ para diferentes fracciones iniciales de llenado.

son más importantes conforme disminuyen el parámetro $\xi$ y la fracción inicial de llenado.

El estudio que se ha llevado a cabo en este capítulo para la ley de aceleración exponencial utilizando el modelo de aguas poco profundas con efectos de reflexión, se puede extender fácilmente para estudiar otras leyes de aceleración y ayudar a elegir los parámetros que optimicen el 
proceso de inyección.

\subsection{Conclusiones}

En este capítulo se ha desarrollado un modelo, basado en la aproximación de aguas poco profundas, que considera los efectos de reflexión del metal fundido contra la pared final de la cámara de inyección. Los resultados de este modelo se han comparado con los del modelo más simple descrito en el capítulo anterior en el que se desprecian estos efectos. Se han estudiado los perfiles de ola de metal fundido en la cámara de inyección, y se han obtenido los parámetros de aceleración que hacen que la ola empiece a romperse justo cuando el metal alcanza la entrada hacia la cavidad del molde. Los resultados así obtenidos muestran una buena concordancia con los valores óptimos del modelo sin reflexión, lo que confirma la validez del modelo más simple para conseguir predicciones cualitativamente correctas de parámetros de aceleración adecuados.

Las predicciones obtenidas respecto a la cantidad de aire atrapado, que se comprobará que presentan una aceptable concordancia cualitativa con los resultados que se expondrán en el Capítulo 4 obtenidos con el código de elementos finitos Wrafts, permiten concluir que el modelo propuesto en este capítulo se puede considerar como una herramienta sencilla y útil para elegir condiciones de operación más apropiadas que reduzcan el aire atrapado a la vez que mantienen el tiempo de llenado tan bajo como sea posible. 


\section{Estudio numérico del movimiento del metal fundido en la cámara de inyección}

En este capítulo se analiza numéricamente el movimiento del metal fundido en el interior de la cámara de inyección durante la fase lenta del proceso de inyección, estudiándose la influencia de los efectos viscosos y no hidrostáticos en el movimiento de la ola de metal fundido para un amplio rango de condiciones de operación. En particular, se tienen en cuenta los efectos de la reflexión del metal fundido contra la pared final y el techo de la cámara de inyección. El análisis numérico se lleva a cabo utilizando un código de elementos finitos que resuelve las ecuaciones de conservación de masa y de cantidad de movimiento utilizando una malla que se mueve y deforma mediante una técnica similar al método LagrangianoEuleriano arbitrario. Para calcular la posición de la superficie libre del metal fundido en cada instante se emplea el método VOF ("volume of fluid"). Los resultados numéricos obtenidos de esta forma se comparan con los resultados obtenidos mediante la aproximación de aguas poco profundas, en los que se desprecian los efectos no hidrostáticos y viscosos, para los perfiles de la superficie libre del metal fundido, los valores óptimos de los parámetros que caracterizan a la ley de movimiento del pistón y el volumen de aire que permanece en la cámara de inyección en 
el instante en el que el metal fundido alcanza la entrada hacia la cavidad del molde. También se comparan algunos resultados numéricos con resultados experimentales para los parámetros de aceleración del pistón que minimizan la cantidad de aire atrapado.

\subsection{Modelo numérico}

\subsubsection{Ecuaciones de conservación y condiciones de contor- no}

A continuación se exponen las ecuaciones de conservación del problema bidimensional que se han resuelto con el código de elementos finitos Wrafts.

Ecuación de conservación de la masa

$$
\frac{\partial u}{\partial x}+\frac{\partial v}{\partial y}=0
$$

Ecuación de conservación de la cantidad de movimiento

$$
\left.\begin{array}{l}
\rho\left(\frac{\partial u}{\partial t}+u \frac{\partial u}{\partial x}+v \frac{\partial u}{\partial y}\right)=-\frac{\partial p}{\partial x}+\mu\left(\frac{\partial^{2} u}{\partial x^{2}}+\frac{\partial^{2} u}{\partial y^{2}}\right) \\
\rho\left(\frac{\partial v}{\partial t}+u \frac{\partial v}{\partial x}+v \frac{\partial v}{\partial y}\right)=-\frac{\partial p}{\partial y}+\mu\left(\frac{\partial^{2} v}{\partial x^{2}}+\frac{\partial^{2} v}{\partial y^{2}}\right)-\rho g
\end{array}\right\}
$$

Ecuación de conservación de la fracción volumétrica de líquido

$$
\frac{\partial F}{\partial t}+\frac{\partial}{\partial x}(u F)+\frac{\partial}{\partial y}(v F)=0
$$

Los métodos de tipo VOF se basan en considerar una función $F$, que se define como la fracción de fluido (en nuestro caso, metal fundido) por unidad de volumen (por unidad de área si el problema es bidimensional), que caracterice las fases: $F=1$ en el metal fundido y $F=0$ en el aire. A partir de dicha función, se define una función discreta $C_{i j}$, análoga a la función discontinua $F$, que representa la fracción volumétrica de metal fundido en la celda $(i, j)$ :

$$
C_{i j} \approx \frac{1}{A_{i j}} \iint_{(i, j)} F(x, y) \mathrm{d} x \mathrm{~d} y .
$$


A partir de la forma geométrica inicial de la superficie libre, se inicializan los valores de $C$, y se prescinde de cualquier información sobre la forma exacta de la interfaz (dada por la distribución de $F$ ). Se tendrá, por tanto, que $0<C<1$ en las celdas que atraviesa la superficie libre, y $C=0$ ó 1 en las restantes. Los algoritmos utilizados en estos métodos constan de dos etapas. En una primera etapa, de reconstrucción de la superficie libre, la forma de ésta en cada celda tiene en cuenta la conservación de volumen local y los valores de $C$ en dicha celda y en celdas adyacentes. En la etapa de propagación, el movimiento de la superficie libre debido al flujo existente se modela utilizando un algoritmo de advección apropiado. Estos métodos de tipo VOF difieren precisamente de los métodos de captura basados en esquemas de advección continua principalmente en la forma de aproximar el término $\vec{v} \cdot \vec{\nabla} F$ de la Ecuación (4.3). Los métodos de seguimiento de volumen lo aproximan geométricamente, basándose en el conocimiento de la posición de la superficie libre reconstruida, de forma que mantienen ésta suficientemente delgada.

Se ha impuesto una condición de no deslizamiento en las paredes de la cámara y se ha fijado la presión en la entrada hacia la cavidad del molde.

\subsubsection{Procedimiento numérico}

El análisis numérico llevado a cabo en este capítulo se ha realizado usando el código Wrafts (Sant y Backer, 1995), el cual está basado en el método de los elementos finitos y utiliza una formulación isoparamétrica lineal para resolver las ecuaciones de conservación de la masa y de la cantidad de movimiento. El problema se ha tratado bidimensionalmente usando una malla de un elemento de espesor, con elementos hexaédricos de 8 nodos, a la que se le ha aplicado condiciones de simetría en sus dos superficies laterales (Figura 4.1). La malla se ha movido y deformado usando una técnica similar al método Lagrangiano-Euleriano arbitrario (Hirt et al., 1974). La ecuación de conservación de cantidad de movimiento se ha resuelto utilizando un método de integración explícito en el tiempo para las velocidades. Se han despreciado los efectos de tensión superficial. La presión se ha calculado a partir de una ecuación de Poisson en cada paso de tiempo, empleando el método de proyección desarrollado por Chorin (1969). Para calcular la posición de la superficie libre se ha utilizado el método VOF (Hirt y Nichols, 1981).

\subsubsection{Estudio de la independencia de la malla}

Para comprobar la influencia de la malla en la solución numérica, se han comparado los resultados de varios casos utilizando diferentes mallas. Como en los restantes casos simulados en este capítulo, se ha utilizado la viscosidad dinámica y la densidad del aluminio puro en su punto de 


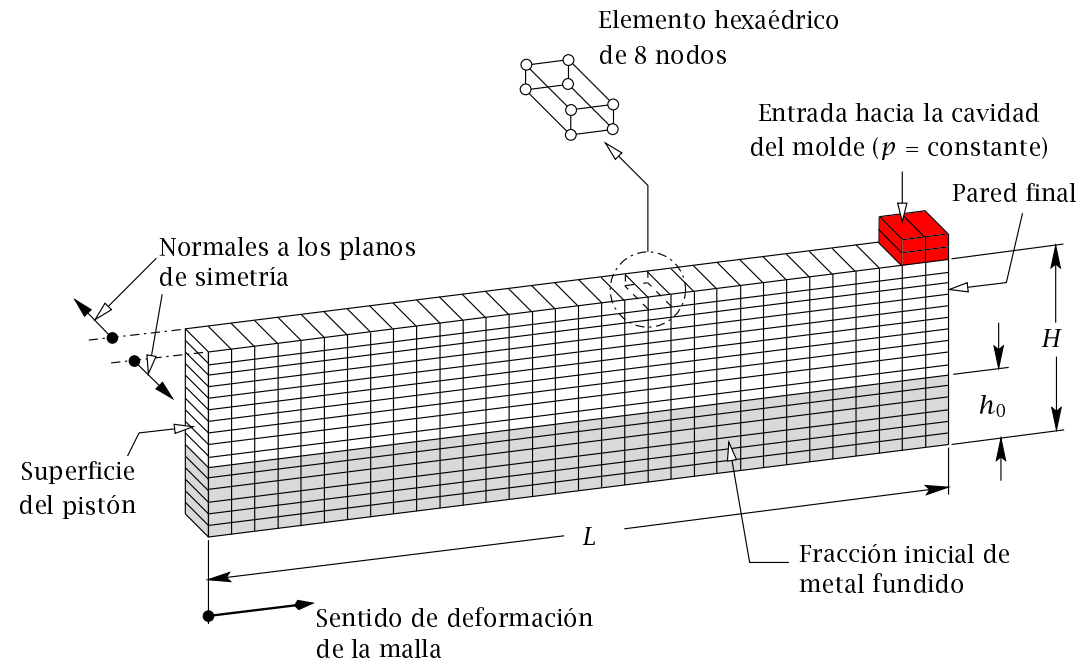

Figura 4.1: Malla de cálculo en la cámara de inyección.

fusión $\left(1,3 \times 10^{-3}\right.$ Pa s y $2385 \mathrm{~kg} \mathrm{~m}^{-3}$, respectivamente). En la Figura 4.2 se representan los perfiles de la superficie libre del metal fundido obtenidos con el código Wrafts para un caso en el que el pistón se mueve según la ley de aceleración de la Ecuación (2.61). La velocidad del pistón se mantiene constante e igual al valor dado por la Ecuación (2.65) para $t \geq t_{\eta}$, con $t_{\eta}$ dado por la Ecuación (2.62), $\eta=5,08 \mathrm{~cm}, h_{0} / \eta=0,254 \mathrm{y}$ $\ell / \eta=9$. Los resultados de esta figura se han obtenido para tres mallas distintas suponiendo que la longitud y la altura de la cámara de inyección son suficientemente grandes con respecto a $\ell$ y $\eta$, por lo que no se consideran los efectos de reflexión del metal fundido contra la pared final ni la interacción del metal con el techo de la cámara de inyección. Se ha encontrado que en general los resultados pueden considerarse independientes de la malla para instantes $t<t_{c}{ }^{*}$ y tamaños de malla en torno a $700 \times 92$ elementos dentro del área $\ell \times \eta$ (lo que corresponde a $1092 \times 120$ elementos en el dominio completo). En instantes posteriores, puede ser necesario utilizar tamaños de malla superiores a $700 \times 92$ para poder obtener resultados independientes de la malla. En la Figura 4.3 se comparan los resultados analíticos y numéricos de los perfiles de la superficie de la ola para un caso en el que el pistón se mueve inicialmente

\footnotetext{
$* t_{c}$ es el instante en el que, según el modelo de ondas simples desarrollado en el Capítulo 2, se formará una discontinuidad en el perfil de la ola de metal fundido, y su valor se puede obtener a partir de las condiciones expresadas en las Ecuaciones (2.11) y (2.12).
} 


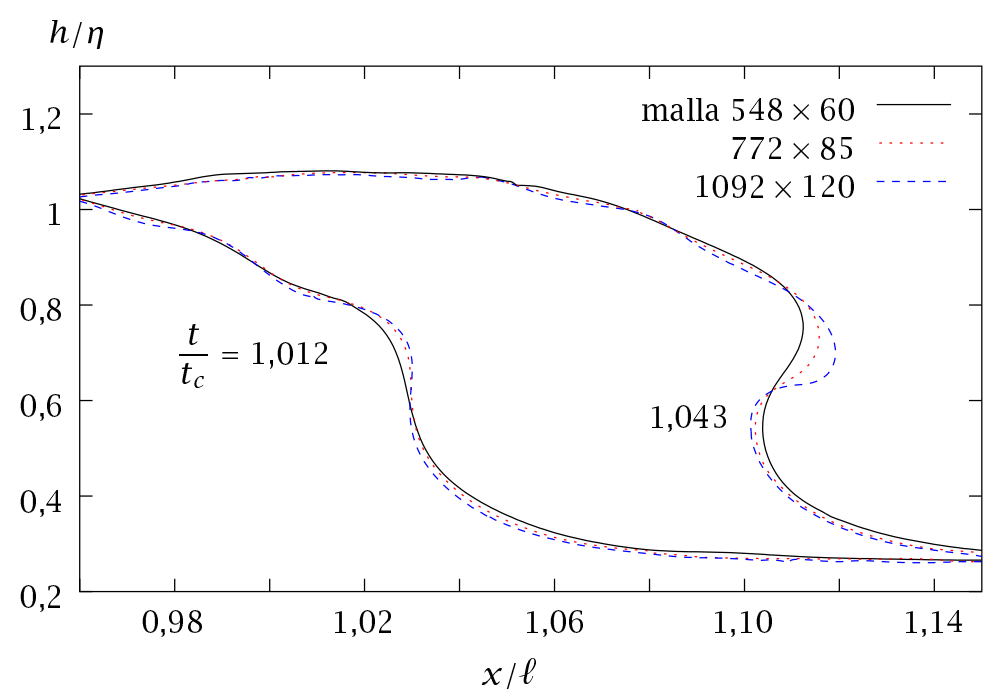

Figura 4.2: Influencia del tamaño de la malla en los perfiles de la superficie libre del metal fundido. Ley de aceleración de la Ecuación (2.61) para $t \leq t_{\eta}$ y $X^{\prime}=u_{\eta}$ para $t>t_{\eta}$, con $\eta=5,08 \mathrm{~cm}, f=0,254, \ell / \eta=9, L / \ell=1,56$ y $H / \eta=1,3$.

con la ley de aceleración de la Ecuación (2.61) y con una velocidad constante dada por la Ecuación (2.65) para $t>t_{\eta}$, con $t_{\eta}$ dado por la Ecuación (2.62) y $f=0,25$. A diferencia de la figura anterior, el parámetro $\ell$ se ha hecho igual a $L$ para tener en cuenta los efectos de reflexión en la pared final de la cámara de inyección. Se puede observar que, como en la figura anterior, los resultados son independientes del tamaño de la malla para valores de éste superiores a $700 \times 92$ elementos $^{\dagger}$, aproximadamente. Para obtener la mayoría de los resultados presentados en esta tesis se ha utilizado este tamaño de malla. Los pasos de tiempo se han variado, normalmente, desde $10^{-3} \mathrm{~s}$ al principio del movimiento del pistón hasta menos de $10^{-5} \mathrm{~s}$ durante la rotura de la ola. Cerca del techo de la cámara de inyección, y para condiciones de operación que favorecen la formación de un chorro a lo largo del techo, es más difícil obtener resultados independientes de la malla ${ }^{\ddagger}$.

${ }^{\dagger}$ Todos los resultados de la Figura 4.3 corresponden a instantes inferiores o iguales a $t_{c}$, el cual, para la ley de aceleración de la Ecuación (2.61) con $\ell=L$, es igual a $L / c_{0}$ (instante en el que, según el modelo analítico sin reflexión, el perfil de la ola es completamente vertical en $x=L$ ).

‡En la Figura 4.10, que posteriormente será comentada, se ha utilizado una malla más fina de $1400 \times 260$ elementos. 


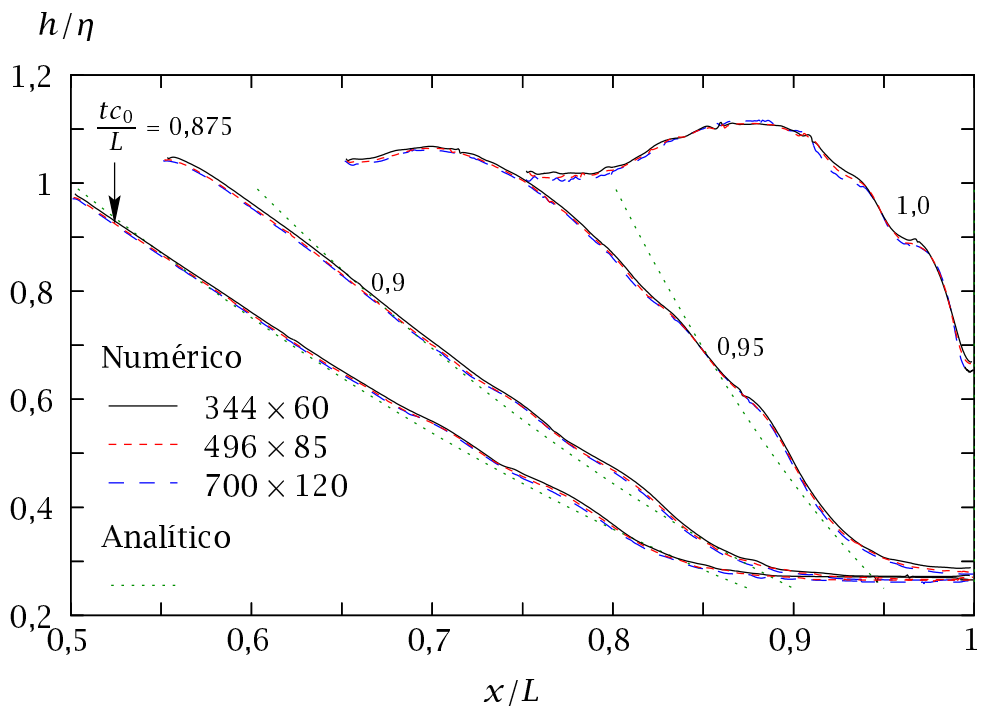

Figura 4.3: Comparación entre los resultados analíticos de los perfiles de la superficie libre de metal fundido en distintos instantes, y los resultados numéricos obtenidos utilizando diferentes tamaños de malla. Ley de aceleración de la Ecuación (2.61) $\operatorname{con} \ell=L$ y $X^{\prime}=u_{\eta}$ para $t>t_{\eta}$, con $\eta=5,08 \mathrm{~cm}$, $\ell / \eta=9, H / \eta=1,3$ y $f=0,25$. 


\subsection{Discusión de resultados}

\subsubsection{Comparación con los modelos de aguas poco profun- das}

Para evaluar la importancia de los efectos viscosos y no hidrostáticos, los resultados para los perfiles de la superficie libre del metal fundido y para los valores del volumen de aire atrapado obtenidos con el código Wrafts se compararán con los del modelo basado en la aproximación de aguas poco profundas que desprecia estos efectos.

\subsubsection{Perfiles de la superficie libre del metal fundido}

Instantes inferiores a $\mathbf{t}_{\mathrm{c}}$. En las Figuras $4.4 \mathrm{a}$ y $4.4 \mathrm{~b}$ se comparan los perfiles de ola obtenidos analíticamente (sin tener en cuenta efectos de reflexión contra la pared final de la cámara) y los obtenidos numéricamente con el código Wrafts. Las condiciones de operación son las mismas que las consideradas en la Figura 4.2, excepto en que corresponden a $H=\eta$ y $f=h_{0} / H=0,5$ en la Figura $4.4 \mathrm{~b}$. De una comparación más detallada con los resultados obtenidos para estas mismas condiciones pero despreciando los efectos viscosos, para los rangos de $t / t_{c}$ incluidos en las Figuras $4.4 \mathrm{a}$ y $4.4 \mathrm{~b}$, se ha encontrado que la influencia de los efectos viscosos se aprecia sólo ligeramente cerca del techo de la cámara de inyección, donde puede formase un chorro en la pared como resultado de la interacción de la ola con el techo. En las Figuras 4.4 a y 4.4 b se puede apreciar que la concordancia entre las predicciones analíticas y los resultados numéricos es muy buena para valores de $t<t_{H}$ (los valores de $t_{H} / t_{c}$ en las Figuras $4.4 \mathrm{a}$ y $4.4 \mathrm{~b}$ son 0,872 y 0,646 , respectivamente), y peor para instantes posteriores, particularmente para $f=0,5$, donde la aproximación de aguas poco profundas, utilizada en el modelo analítico, está menos justificada.

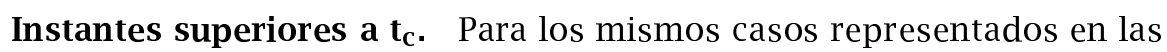
Figuras $4.4 \mathrm{a}$ y $4.4 \mathrm{~b}$, en las Figuras $4.5 \mathrm{a}$ y $4.5 \mathrm{~b}$ se representan los perfiles de la superficie libre de la ola para $t \geq t_{c}$. Los resultados numéricos del código Wrafts se obtuvieron en primer lugar despreciando los efectos viscosos, considerándolos posteriormente. Los resultados obtenidos se comparan en las Figuras 4.5a y 4.5b. En estas figuras se puede apreciar un chorro en la pared a lo largo del techo de la cámara de inyección. Este chorro se forma a pesar de que la aceleración del pistón cesa en $t=t_{H}$ y su caída sobre la superficie libre puede contribuir a aumentar la ingestión de aire y acelerar la rotura de la ola (Figura 4.5b). Se puede observar, especialmente para $f=0,254$, que cuando los efectos viscosos 

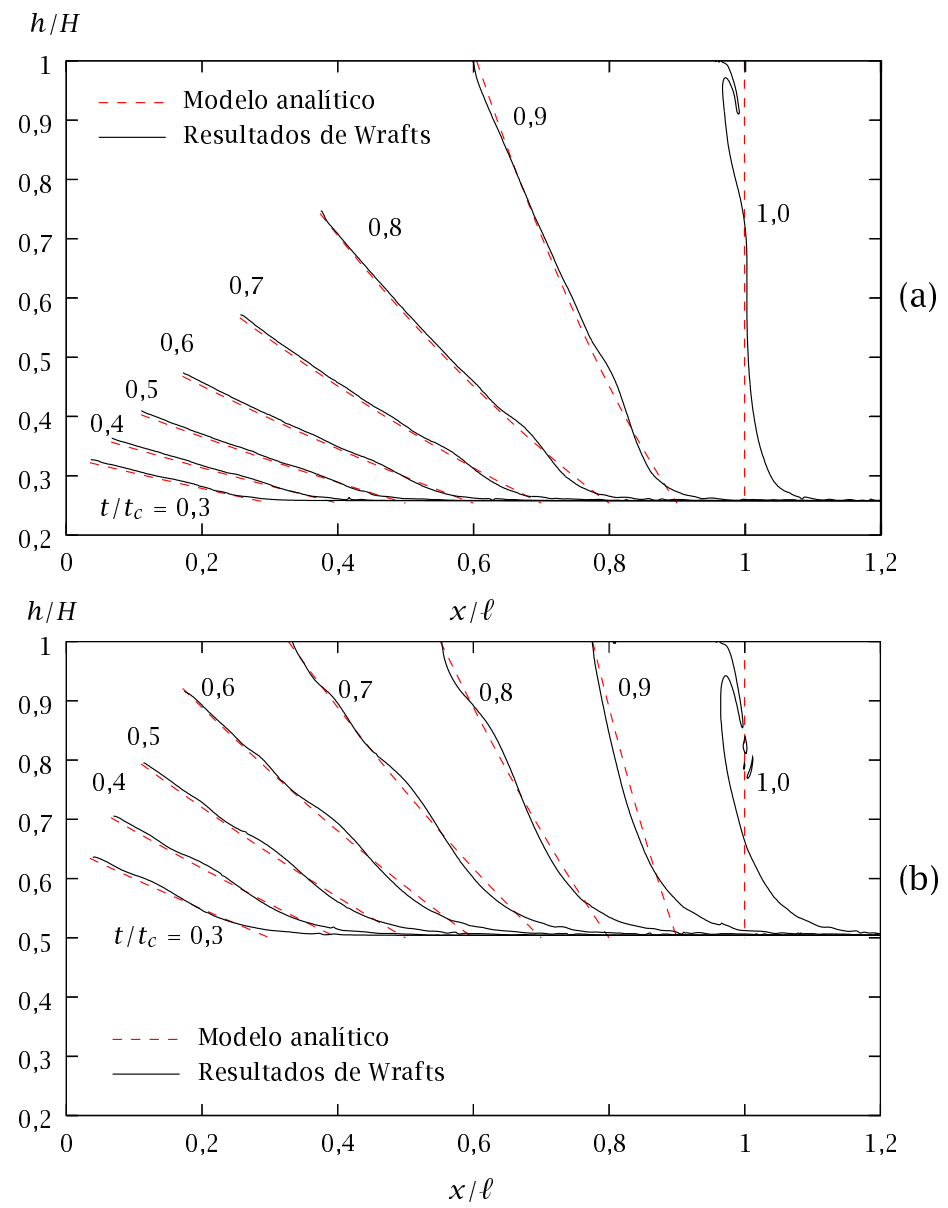

(b)

Figura 4.4: Comparación entre resultados numéricos y analíticos para los perfiles de la superficie libre. Ley de aceleración del pistón de la Ecuación (2.61) para $t \leq t_{\eta}$ y $X^{\prime}=u_{\eta}$ para $t>t_{\eta}$. $\eta=5,08 \mathrm{~cm}, \ell / \eta=9, L / \ell=1,56, H / \eta=1$. a) $f=0,254$. b) $f=0,5$. 


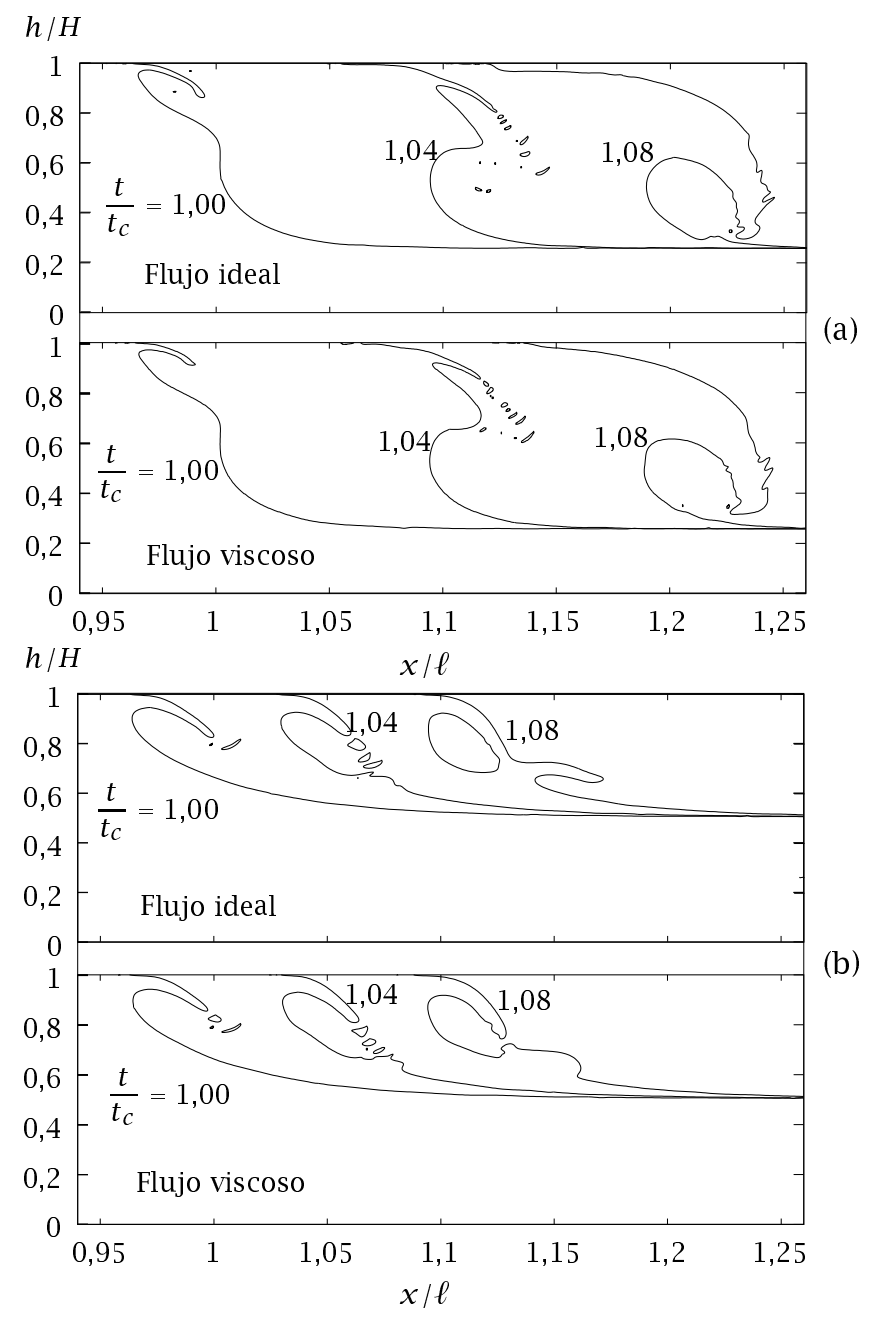

Figura 4.5: Resultados numéricos de los perfiles de la ola para la ley de aceleración de la Ecuación (2.61) $\left(t \leq t_{\eta}\right)$ y $X^{\prime}=u_{\eta}$ para $t>$ $t_{\eta}$, con $t \geq t_{c}, \eta=5,08 \mathrm{~cm}, \ell / \eta=9, L / \ell=1,56, H / \eta=1$. a) $f=0,254$. b) $f=0,5$. 
son despreciables, la distancia que el chorro recorre junto al techo es ligeramente superior.

De la Figura 4.5 se deduce que el modelo numérico predice el inicio de la rotura de la ola en un instante posterior al tiempo $t_{c}$ obtenido analíticamente. El retraso es muy pequeño para $f=0,254$, y aumenta a medida que la fracción inicial de llenado es mayor. Los resultados para flujo ideal presentados en la Figura 4.5b para $f=0,5$ muestran que la ola no empezará a romperse antes de $t=1,08 t_{c}$; sin embargo, el desarrollo más complejo del flujo provocado por los efectos del chorro que se forma en el techo de la cámara de inyección puede provocar que la ola se rompa antes. La evolución del chorro de metal fundido junto al techo de la cámara de inyección debe ser estudiado de forma más detallada, incluyendo una comprobación más cuidadosa de la independencia de la malla para instantes superiores a $t_{c}$.

\subsubsection{Cierre de la entrada hacia la cavidad del molde}

En las Figuras 4.6a y 4.6b se comparan, para la ley de movimiento del pistón de la Ecuación (2.17), los resultados numéricos de los perfiles de la superficie libre del metal fundido obtenidos con el código Wrafts y los obtenidos con el modelo basado en la aproximación de aguas poco profundas en el que se consideran los efectos de reflexión en la pared final de la cámara de inyección, para $L / H=9$ y fracciones iniciales de llenado de 0,25 y 0,4 , respectivamente. Para cada valor de $f$, los perfiles representados corresponden a dos combinaciones diferentes de los parámetros adimensionales $\xi$ y $L / \beta$, satisfaciendo en ambos casos la condición de que, según el modelo de aguas poco profundas, la ola reflejada contra la pared final de la cámara de inyección empiece a romperse justo en el instante en el que el metal fundido alcanza la entrada hacia la cavidad del molde. Para cada uno de los casos representados en la Figura 4.6 , este instante es el que corresponde al valor de $t / t_{f}$ que se ha representado en último lugar, siendo $t_{f}$ el tiempo de llenado de la cámara de inyección. En los resultados presentados anteriormente se puede observar que la concordancia entre ambos tipos de resultados es buena para distancias suficientemente alejadas de la pared final de la cámara de inyección, aunque los resultados numéricos obtenidos con el código Wrafts predicen un ligero retraso en la rotura de la ola y perfiles de la superficie libre del metal fundido más suaves. Cerca de la pared final de la cámara, la reflexión del metal fundido acentúa las diferencias entre los resultados de ambos modelos, especialmente en lo que se refiere a la predicción del instante en el que el metal fundido alcanza la entrada hacia la cavidad del molde, sobre todo para grandes valores de $\xi$. En general, los resultados numéricos tienden a retrasar el instante en el que el metal fundido alcanza la entrada hacia la cavidad del molde. 

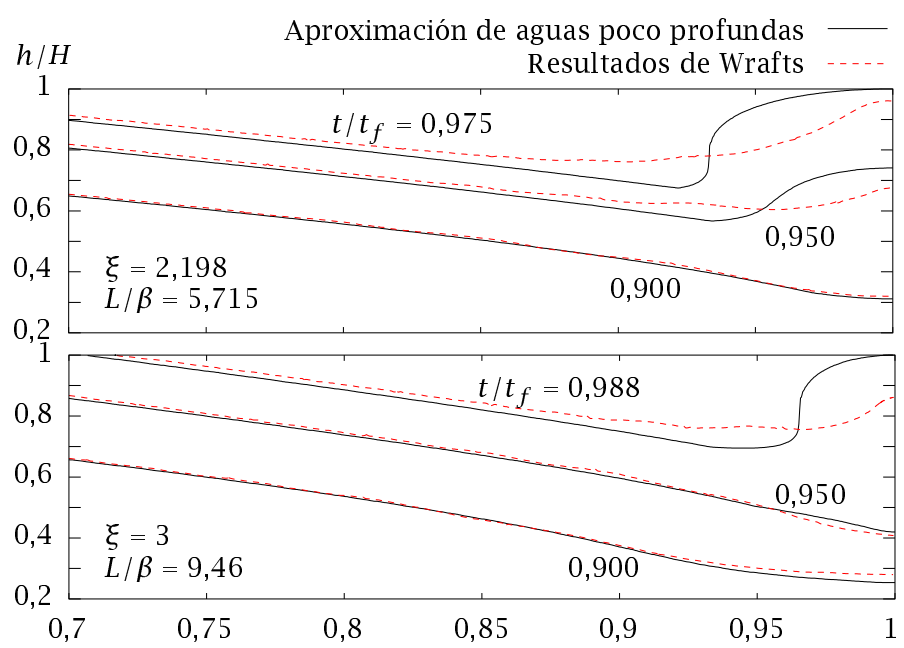

(a)

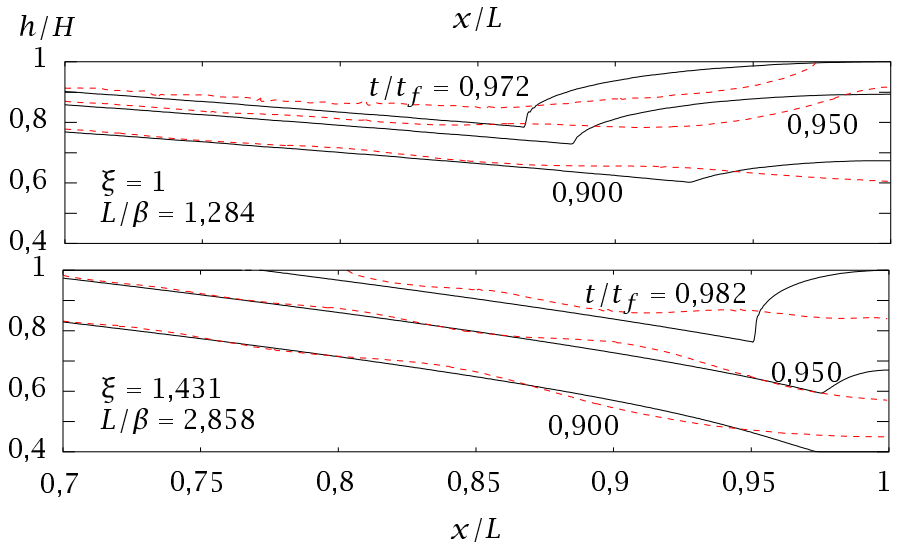

(b)

Figura 4.6: Comparación entre los resultados de los perfiles de ola obtenidos con el modelo de aguas poco profundas, para condiciones que hacen que la ola reflejada empiece a romperse en el instante en el que el metal fundido alcanza la entrada hacia la cavidad del molde, y los correspondientes resultados numéricos obtenidos con el código Wrafts. a) $f=0,25$, b) $f=0,4$. 


\subsubsection{Volumen de aire atrapado}

Aceleración del pistón exponencial. Para la ley de movimiento del pistón de la Ecuación (2.17), en las Figuras 4.7 y 4.8, que corresponden a fracciones iniciales de llenado de 0,25 y 0,4 , respectivamente, se representan los resultados obtenidos con el modelo de aguas poco profundas y con el código Wrafts para los valores adimensionales del área ocupada por el aire en la cámara de inyección cuando el metal fundido alcanza la entrada hacia la cavidad del molde, $A /(H L)$, en función de $L / \beta$, para diferentes valores de $\xi$. Se espera que la cantidad de aire finalmente atrapado esté relacionada con $A$. En la parte superior de estas figuras, también se ha representado el tiempo adimensional de llenado $t_{f} \mathcal{C}_{0} / L$.

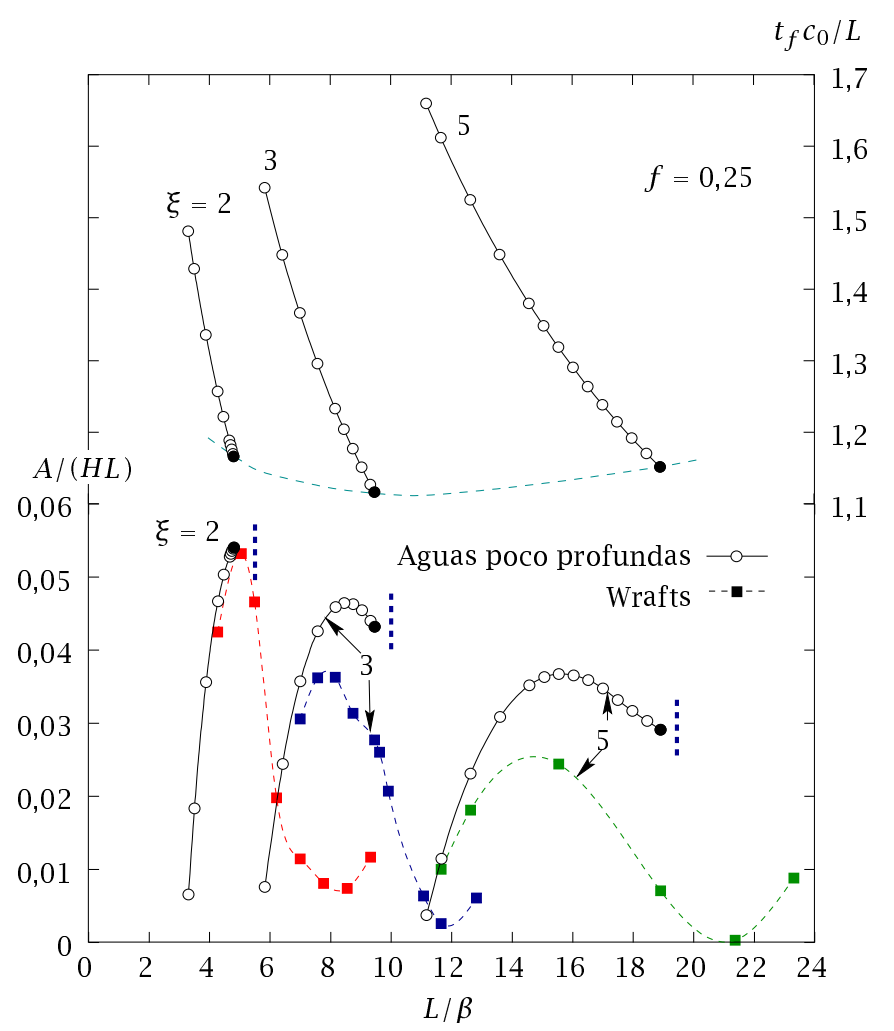

Figura 4.7: Resultados del área adimensional ocupada por el aire cuando el metal fundido alcanza la entrada hacia la cavidad del molde, obtenidos con el código Wrafts y con el modelo de aguas poco profundas, y del tiempo adimensional de llenado en función de $L / \beta$, para diferentes valores de $\xi, L / H=9$ y $f=0,25$. 


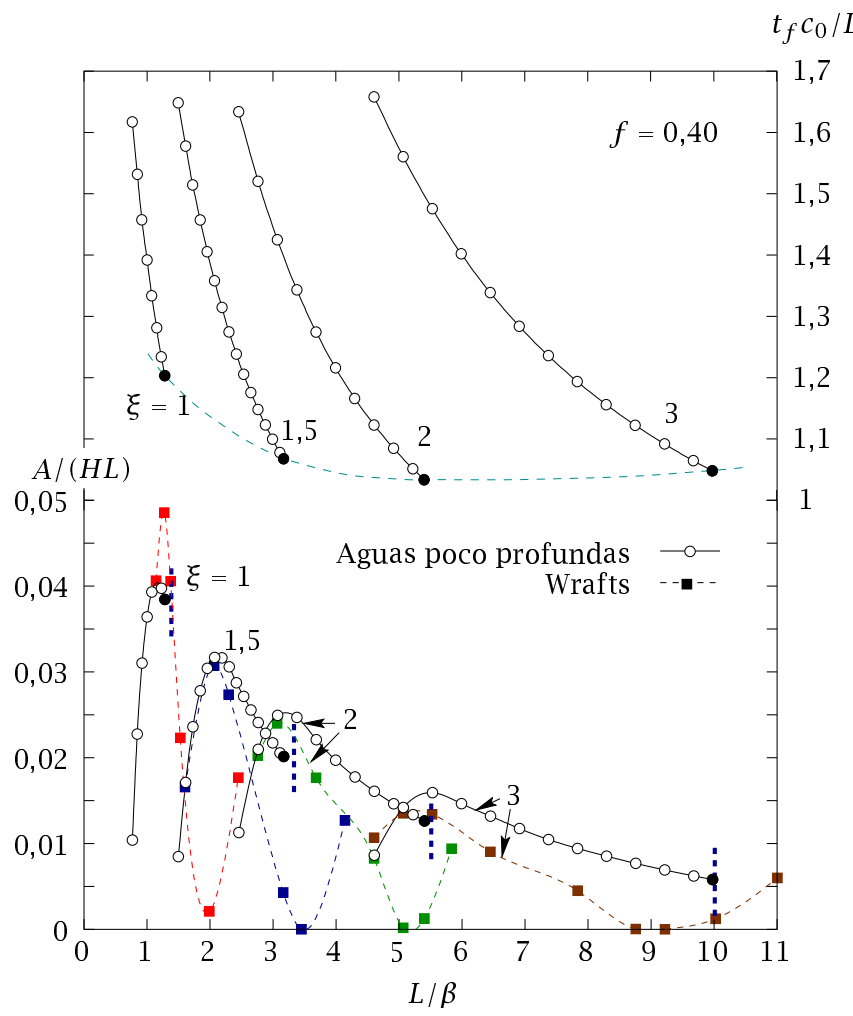

Figura 4.8: Resultados del área adimensional ocupada por el aire cuando el metal fundido alcanza la entrada hacia la cavidad del molde, obtenidos con el código Wrafts y con el modelo de aguas poco profundas, y del tiempo adimensional de llenado en función de $L / \beta$, para diferentes valores de $\xi, L / H=9$ y $f=0,4$.

Los resultados numéricos obtenidos con el código Wrafts, representados en las Figuras 4.7 y 4.8, muestran una buena concordancia cualitativa con los resultados obtenidos con el modelo basado en la aproximación de aguas poco profundas. Sin embargo, en general, el código Wrafts predice cantidades más pequeñas de aire atrapado, particularmente, para grandes valores de $\xi$, excepto para altas fracciones iniciales de llenado y bajos valores de $\xi$. También se puede apreciar que, en los resultados de Wrafts, el máximo local de $A /(H L)$ está ligeramente desplazado hacia valores más bajos de $L / \beta$, excepto para valores grandes de $f$ y valores pequeños de $\xi$. Por otro lado, para un valor dado de $\xi$, los resultados numéricos obtenidos con el código Wrafts muestran que la cantidad de aire atrapado presenta un mínimo local para un cierto valor de $L / \beta$, por 
encima del cual $A /(H L)$ aumenta con $L / \beta$, lo que está de acuerdo con la hipótesis adoptada en el Capítulo 3 por la que se suponía que para valores de $L / \beta>(L / \beta)_{\text {opt }}$ el aire atrapado aumentaría. Sin embargo, este mínimo local se alcanza para un valor de $L / \beta$ generalmente mayor que $(L / \beta)_{\text {opt }}$, por lo que la cantidad de aire atrapado calculada con el modelo CFD será mínima para mayores aceleraciones que las predichas por el modelo basado en la aproximación de aguas poco profundas (valores de $\alpha^{2} \beta$ en torno a un 20\% más grandes). Obsérvese, sin embargo, que los resultados numéricos obtenidos con Wrafts que se han representado en la Figura 4.8 para $f=0,4$ y valores de $\xi=2$ y 3 , presentan un mínimo local de $A /(H L)$ para un valor de $L / \beta$ ligeramente menor que $(L / \beta)_{\text {opt }}$, lo que es debido a la formación de chorros a lo largo del techo de la cámara de inyección que anticipan la rotura de la ola y el cierre de la entrada hacia la cavidad del molde. En la Figura 4.9 se han representado los valores del área adimensional y los tiempos de llenado correspondientes a los mínimos locales encontrados numéricamente con el código Wrafts y representados en las Figuras 4.7 y $4.8^{\S}$. Se puede observar, con más claridad en la Figura 4.9b, que, en el rango representado en estas figuras, existe un valor del parámetro $\xi$, que se encuentra alrededor de 5 y 1,5 para $f=0,25$ y 0,4 , respectivamente, por encima del cual no se consigue una reducción apreciable de aire y sí un aumento del tiempo de llenado, por lo que dichos valores de $\xi$ se pueden considerar como una elección apropiada para minimizar el aire atrapado reduciendo, en lo posible, el tiempo de llenado.

Las discrepancias entre los resultados numéricos obtenidos con Wrafts y los del modelo simplificado reflejadas en las Figuras 4.7 y 4.8 se deben en general al retraso del metal fundido, predicho por el código Wrafts, en alcanzar la entrada hacia la cavidad del molde para grandes valores de $\xi$, lo cual se puede observar en los perfiles de la Figura 4.6. Obsérvese, sin embargo, que esto no sucede para valores de $\xi$ suficientemente pequeños y valores de $f$ grandes (ver, por ejemplo, los perfiles de la superficie libre del metal fundido en la parte superior de la Figura 4.6b), para los que el código Wrafts predice un aumento más rápido de la altura de la superficie libre en la pared final de la cámara.

Aceleración del pistón dada por la Ecuación (2.61). También se ha evaluado, utilizando el código Wrafts, la predicción de atrapamiento de aire nulo del modelo analítico para la ley de aceleración de la Ecuación (2.61)

\footnotetext{
§En la Figura 4.9 se puede observar que los tiempos de llenado correspondientes a los mínimos locales del área encontrados numéricamente con el código Wrafts no siguen la misma tendencia que los de la Figura 3.16 correspondiente al modelo de aguas poco profundas con efectos de reflexión. Esto se debe al desplazamiento de los mínimos locales de $A /(H L)$ obtenidos con el modelo CFD hacia valores más grandes de $L / \beta$, especialmente para pequeños valores de $f$ y $\xi$.
} 


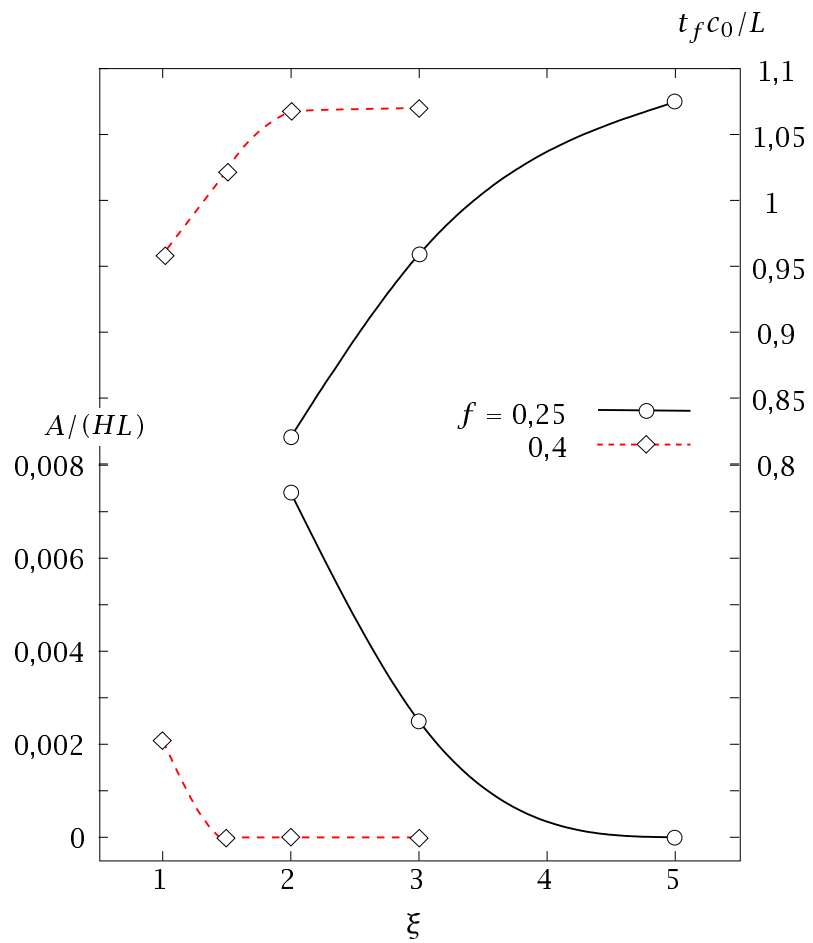

Figura 4.9: Valores del área mínima adimensional $A /(H L)$ ocupada por el aire cuando el metal fundido alcanza la entrada hacia la cavidad del molde, obtenidos con el código Wrafts, y del tiempo adimensional de llenado en función de $\xi$ con $L / H=$ 9. a) $f=0,25$. b) $f=0,4$. 
con $\ell=L$ para $t \leq t_{H}$ y $X^{\prime}=u_{H}$ para $t>t_{H}$. Como ejemplo, en las Figuras $4.10 \mathrm{a}$ y $4.10 \mathrm{~b}$ se representan los resultados numéricos obtenidos para la superficie libre del metal fundido, en tres instantes diferentes, para $f=0,4$ y 0,25 , respectivamente, $\operatorname{con} L / H=6$ y $H=5,08 \mathrm{~cm}$, utilizando una malla de $1400 \times 260$ elementos. El tamaño de la entrada hacia la cavidad del molde se ha considerado de tamaño muy pequeño, suponiendo además que el flujo de aire a través del conducto que comunica la cámara de inyección con el molde es sin fricción. Los valores del área adimen-
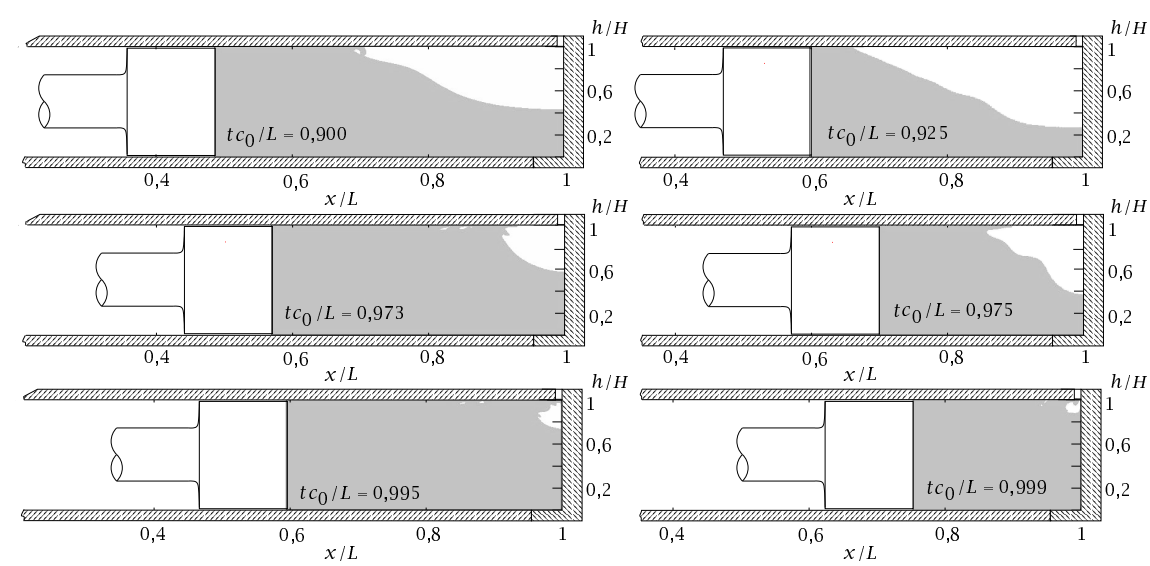

(a)

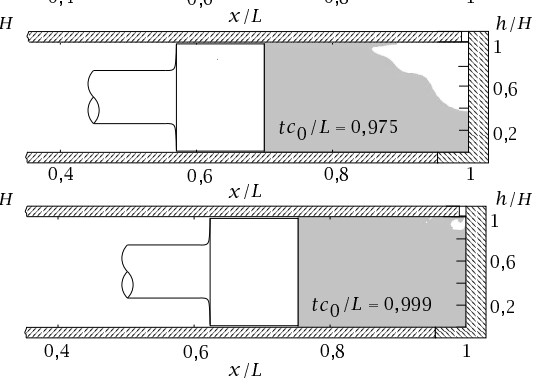

(b)

Figura 4.10: Resultados numéricos de la evolución de la superficie libre del metal fundido para la ley de aceleración de la Ecuación (2.61) con $\ell=L$ y $L / H=6$. a) $f=0,4$. b) $f=0,25$.

sional ocupada por el aire atrapado en el último instante representado en las Figuras $4.10 \mathrm{a}$ y $4.10 \mathrm{~b}$ son $A /(H L)=8 \times 10^{-3}$ y $8,3 \times 10^{-4}$, respectivamente, los cuales pueden considerarse aceptablemente próximos a las predicciones analíticas de aire atrapado nulo. Los resultados numéricos de $A /(H L)$ para diferentes valores de $f$ y $L / H$, aunque del mismo orden de magnitud que los correspondientes a los de la Figura 4.10, se ha comprobado que son muy sensibles a los efectos debidos a la formación de un chorro a lo largo del techo de la cámara de inyección. Por lo tanto, una determinación precisa de la cantidad de aire atrapado requeriría un modelado detallado de los complejos mecanismos de atrapamiento de aire que pueden aparecer, con más probabilidad al final de la fase lenta, en el flujo dentro de la cámara de inyección y en la entrada hacia la cavidad del molde. La ley de aceleración de la Ecuación (2.61) aumenta la tendencia a la formación de chorros en el techo de la cámara, tal y como 
se aprecia en la Figura 4.10.

\subsubsection{Límites de la fracción inicial de llenado}

En las Figuras 2.17 y 4.3 se pueden apreciar los efectos de la reflexión de la ola contra la pared final de la cámara y ver con más detalle la influencia de los efectos no hidrostáticos una vez que el pistón alcanza la velocidad crítica $u_{\eta}$. Se puede observar que cuando el pistón deja de ser acelerado la superficie libre del metal fundido tiende a alcanzar una altura superior a $\eta$, lo que podría provocar que el metal fundido salga fuera de la cámara de inyección a través del orificio de colada si la posición $X_{p}$ de su extremo no satisface la condición expresada en la Ecuación (2.66). Estos efectos también se pueden observar en la Figura 4.11, en la que se representan los resultados numéricos de los perfiles de la superficie de la ola en tres instantes distintos para un caso con la ley de aceleración del pistón de la Ecuación (2.61) con $\ell=L$, y condiciones para las que la superficie libre alcanza una altura ligeramente superior a $H$ en el orificio de colada y provoca que el metal fundido salga fuera de la cámara de inyección. Esto ocurre a pesar de que la velocidad del pistón se mantiene constante e igual a la velocidad crítica $u_{H}$ para $t>t_{H}$. En esta figura también se han representado los resultados analíticos de los perfiles de la superficie libre, cuya concordancia con los resultados numéricos es muy buena, como se puede observar.

En la Figura 4.12 se comparan las predicciones analíticas y numéricas de la altura del metal fundido en la superficie del pistón en función del tiempo adimensional $t / t_{H}$, para diferentes fracciones iniciales de llenado, con la ley de aceleración del pistón de la Ecuación (2.61). Para este caso, la concordancia entre los resultados analíticos y numéricos es también muy buena. Aunque el modelo analítico, obviamente, no puede describir el flujo de metal fundido a través del orificio de colada, la comparación anterior sugiere que este modelo se puede emplear para determinar de forma precisa las condiciones de operación necesarias que eviten que el metal fundido salga fuera de la cámara de inyección. Por lo tanto, los resultados del modelo analítico obtenidos en el Capítulo 2 para determinar los valores límite de la fracción inicial de llenado que permiten evitar que el metal fundido salga fuera de la cámara de inyección a través del orificio de colada, se pueden considerar como una buena aproximación.

\subsubsection{Comparación con resultados experimentales}

Los valores del parámetro de aceleración $\alpha$ que minimizan la cantidad de aire atrapado obtenidos experimentalmente por Duran et al. (1991) para distintas fracciones iniciales de llenado y un parámetro $L / \beta$ fijo e igual a 18 , se comparan en esta sección con los obtenidos numérica y 

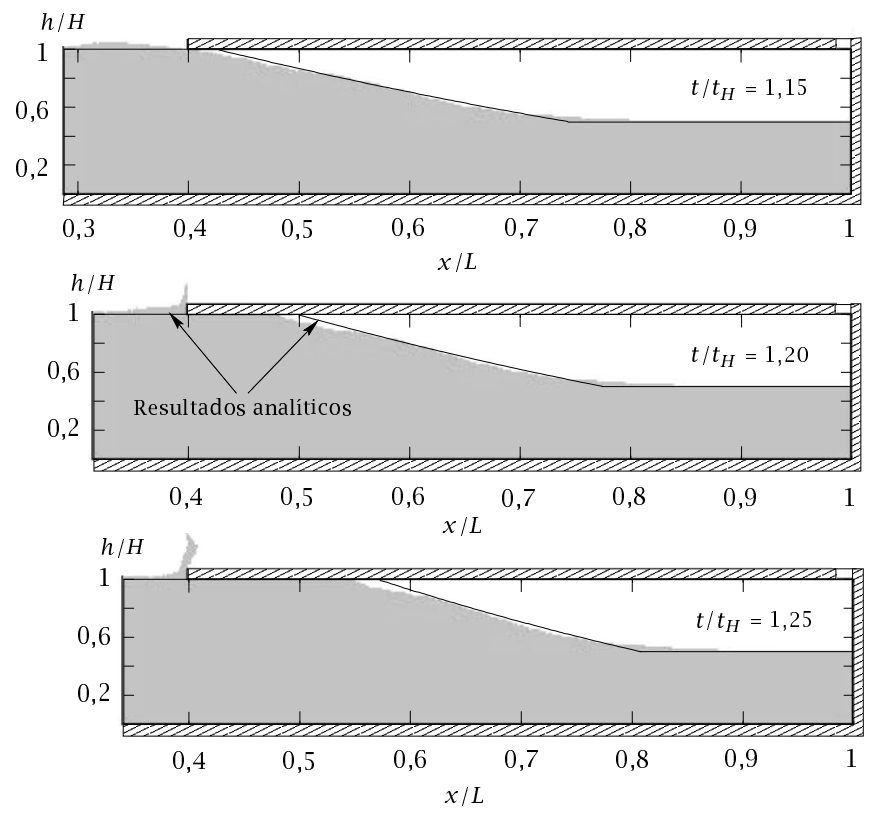

Figura 4.11: Comparación entre los resultados analíticos (línea continua) y numéricos (contorno gris) de los perfiles de la superficie libre en distintos instantes. Ley de aceleración de la Ecuación (2.61) con $\ell=L$ para $t \leq t_{H}, X^{\prime}=u_{H}$ para $t>t_{H}, f=0,5, L / H=9$ y $X_{p} / L=0,4$. 


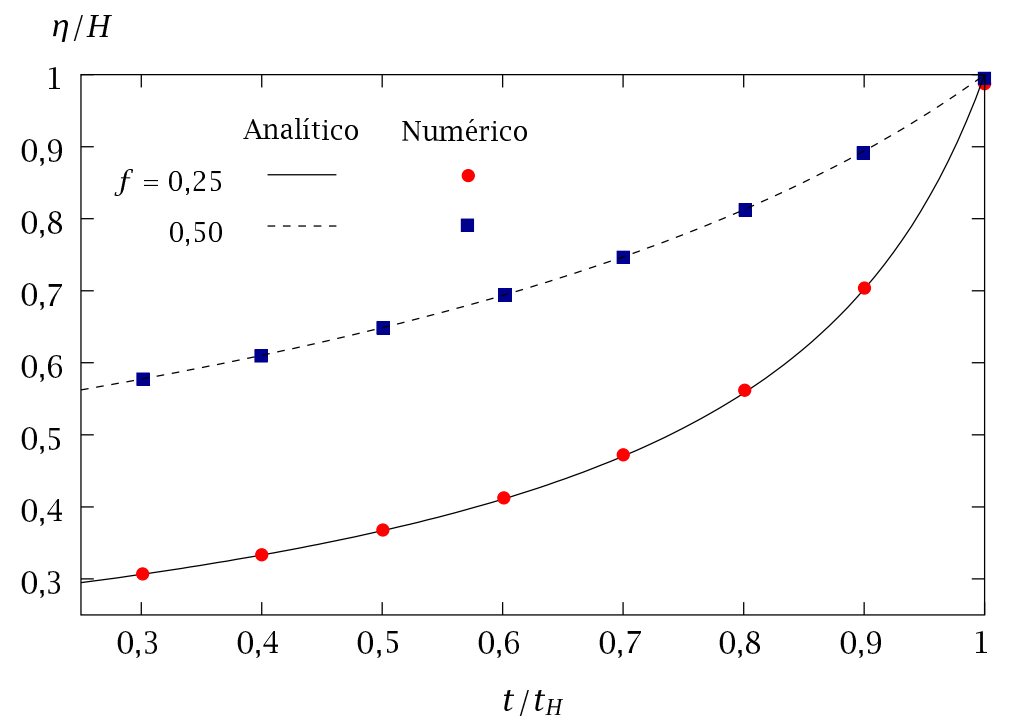

Figura 4.12: Comparación entre los resultados analíticos y numéricos de la altura del metal fundido en la superficie del pistón en función del tiempo $\left(t \leq t_{H}\right)$, para diferentes fracciones iniciales de llenado, con la ley de aceleración de la Ecuación (2.61) para $\ell=L$, y $L / H=9$. 
analíticamente. En las Figuras 4.13 y 4.14 se representan los perfiles de la superficie libre del metal fundido para la ley de aceleración del pistón de la Ecuación (2.16), con $f=0,254$ y $f=0,5$, respectivamente, $L / \beta=18, L / H=9, H=5,08 \mathrm{~cm}$ y diferentes valores de $\alpha$. En estas figuras, el tiempo se ha adimensionalizado con el tiempo de llenado $t_{f}$. El último tiempo representado en las Figuras 4.13 y 4.14 corresponde al instante en el que la superficie libre del metal fundido, obtenida con el código Wrafts, alcanza la entrada hacia la cavidad del molde. Se

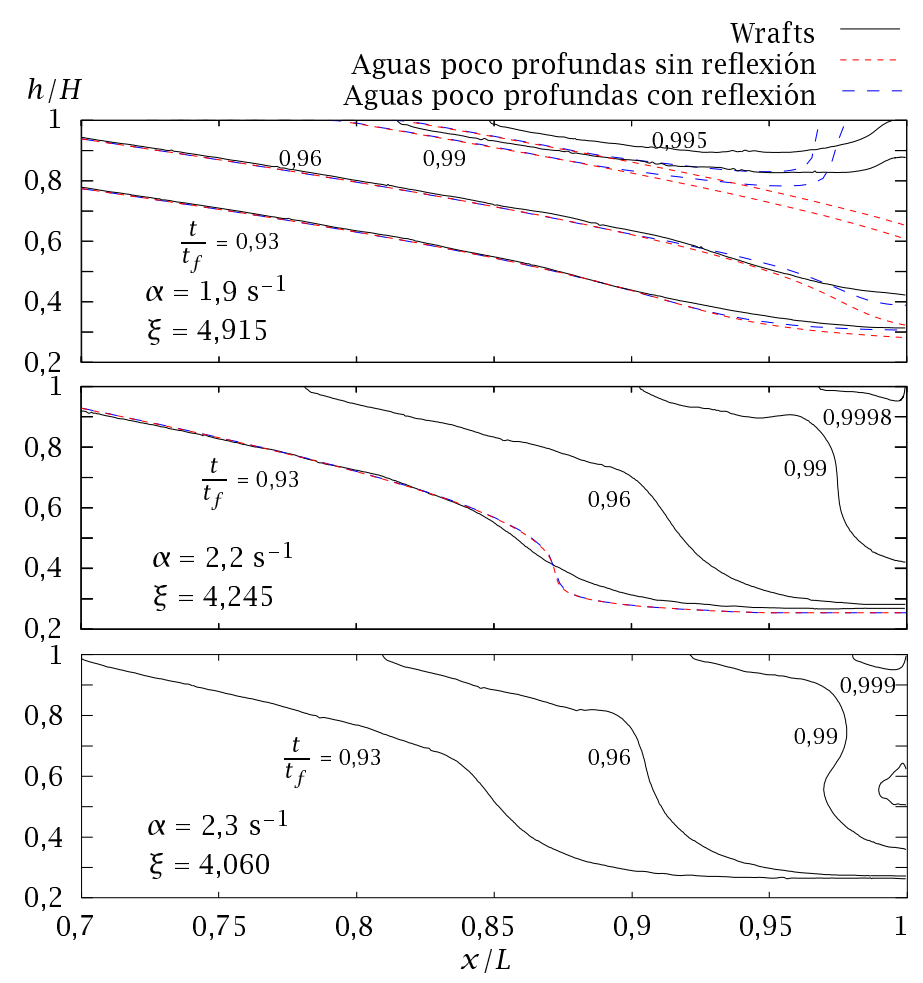

Figura 4.13: Resultados numéricos y analíticos de los perfiles de la superficie libre del metal fundido para la ley de aceleración de la Ecuación (2.16). $L / \beta=18, L / H=9, H=5,08 \mathrm{~cm}$, $f=0,254$ y diferentes valores de $\alpha$.

puede ver que el chorro del techo sólo aparece en la Figura 4.14 para casos en los que $f=0,5$, con valores suficientemente altos de $\alpha$. En esta figura, los perfiles correspondientes a la superficie libre del metal fundido obtenidos mediante los modelos basados en la aproximación de aguas poco profundas se representan con líneas discontinuas para valores de $t \leq t_{c}$ (por ejemplo, para $f=0,254$ y $\alpha=2,3 \mathrm{~s}^{-1}, t_{c} / t_{f}<0,93$, 

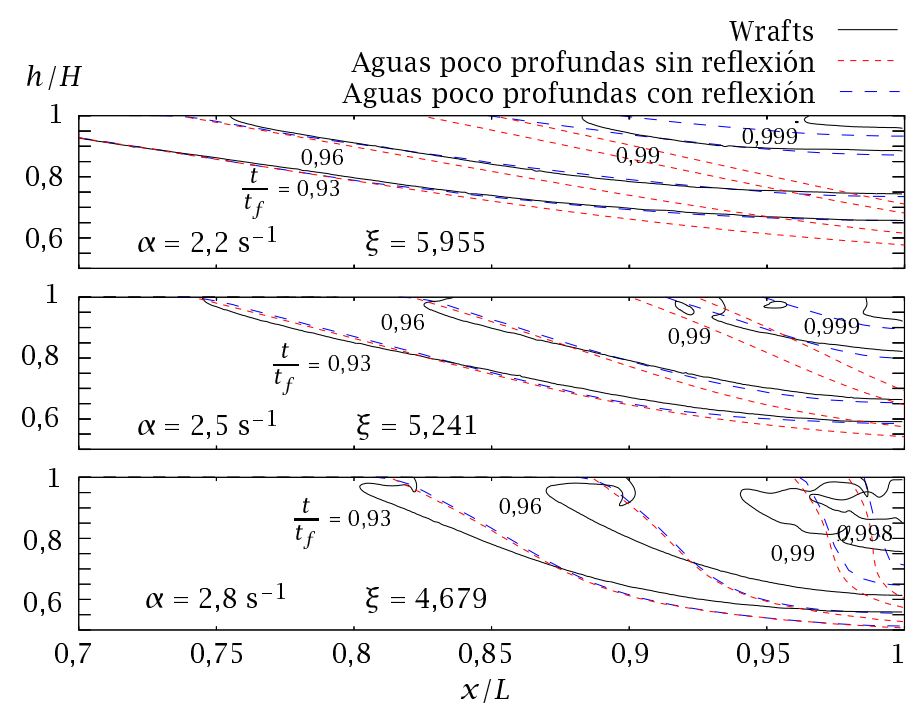

Figura 4.14: Resultados numéricos y analíticos de los perfiles de la superficie libre del metal fundido para la ley de aceleración de la Ecuación (2.16). $L / \beta=18, L / H=9, H=5,08 \mathrm{~cm}$, $f=0,5$ y diferentes valores de $\alpha$.

por lo que en este caso no se representan resultados). Obsérvese que la concordancia global entre los resultados de los modelos de los capítulos anteriores y los numéricos es aceptable.

De los resultados numéricos presentados en las Figuras 4.13 y 4.14 , se puede determinar un valor del parámetro de aceleración del pistón, $\alpha$, para $L / \beta=18$, que minimice el volumen de aire atrapado en función de la fracción inicial de llenado. En la Figura 4.15 se comparan los valores de la aceleración del pistón obtenidos de esta forma y las predicciones analíticas obtenidas cuando se impone la condición de que el frente de la ola de metal fundido empiece a romperse exactamente en la pared final de la cámara de inyección, sin tener en cuenta efectos de reflexión" (no se han representado los resultados correspondientes al modelo de aguas poco profundas teniendo en cuenta efectos de reflexión por ser muy próximos a los obtenidos despreciando estos efectos; véase la Figura 3.17, a partir de la cual se pueden obtener, junto con las Ecuaciones (2.33) y (2.35), los parámetros de aceleración para un $L / \beta=18$ y distintas fracciones iniciales de llenado que satisfacen la condición de que el perfil de la ola empiece a romperse justo cuando el metal fundido al-

\footnotetext{
"Recuérdese que, según el modelo analítico sin reflexión, y teniendo en cuenta la Ecuación (2.33), el valor de $\xi$ que satisface esta condición para $L / \beta=18$ es igual a 4,695 siempre que $f \leq f^{*}=0,652$.
} 
canza la entrada hacia la cavidad del molde). Analíticamente se puede deducir que, para las variables de proceso de las Figuras 4.13 y 4.14 , la discontinuidad en la ola debería aparecer en una sección localizada entre el pistón y el extremo más avanzado de la ola, y por lo tanto, la condición mencionada produciría la reflexión de la ola antes de que empiece a romperse en $x=L$, como ocurre en los resultados que tienen en cuenta efectos de reflexión de las Figuras 4.13 y 4.14. Como se puede ver en la Fi-

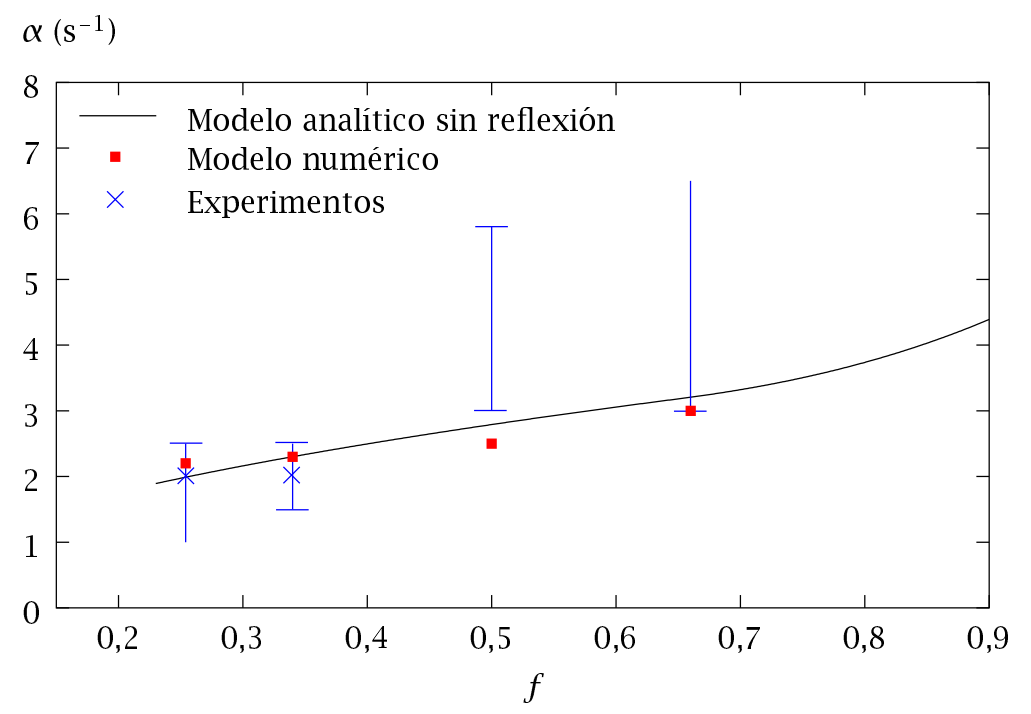

Figura 4.15: Comparación entre resultados analíticos, numéricos y experimentales para el parámetro de aceleración óptimo del pistón, $\alpha$, en función de la fracción inicial de llenado con $L / \beta=18, L / H=9$ y $H=5,08 \mathrm{~cm}$.

gura 4.15, la concordancia entre las predicciones numéricas y analíticas es muy buena. La concordancia con las mediciones experimentales de Duran et al. (1991), presentadas en la Figura 2.9, también es buena a pesar de las incertidumbres existentes en la comparación, lo cual se debe, en parte, al hecho de que la cámara de inyección en los experimentos es de sección circular. En la Figura 4.15 se utilizaron valores equivalentes para la fracción inicial de llenado bidimensional. Como ya se indicó en el Capítulo 2, para $f=0,254,0,5$ y 0,66, las mediciones experimentales no permiten identificar claramente el volumen mínimo de aire atrapado en el rango de aceleraciones medidas. Al igual que en la Figura 2.8, los rangos de los posibles valores del óptimo $\alpha$ se indican en la Figura 4.15 mediante líneas verticales.

Aunque este trabajo se centra principalmente en condiciones próximas 
a las óptimas, para las que el análisis numérico que se presenta es adecuado, también se debería estudiar el problema tridimensionalmente y analizar efectos turbulentos y de tensión superficial, en especial respecto a su influencia en el chorro formado en el techo de la cámara de inyección y en los mecanismos de atrapamiento de aire.

\subsection{Conclusiones}

Se ha encontrado que, para condiciones en las que se espera que los efectos viscosos y no hidrostáticos sean despreciables, existe una muy buena concordancia entre las predicciones obtenidas con el modelo numérico descrito y los resultados del modelo de aguas poco profundas. Los efectos no hidrostáticos se ponen más claramente de manifiesto una vez que la superficie libre del metal fundido alcanza el techo de la cámara de inyección y la velocidad del pistón se mantiene constante. Los resultados numéricos predicen un ligero retraso en la rotura de la ola de metal fundido con respecto a las predicciones analíticas, particularmente, para grandes fracciones iniciales de llenado. Se ha encontrado que, bajo ciertas condiciones de operación, aparece un chorro junto al techo de la cámara de inyección como resultado de la interacción que tiene lugar entre éste y la parte superior del frente de la ola, y que la caída de este chorro sobre la superficie libre del metal fundido puede tender a anticipar la rotura de la ola. Los efectos viscosos deberían ser tenidos en cuenta para modelar apropiadamente este chorro. Para casos en los que el pistón es acelerado según la ley exponencial en el tiempo, los resultados numéricos muestran una buena concordancia con los resultados del modelo de aguas poco profundas para los perfiles de la ola, excepto cerca de la pared final de la cámara de inyección, donde existen discrepancias entre los instantes en los que el metal fundido alcanza la entrada hacia la cavidad del molde, particularmente para grandes valores del parámetro $\xi$. Estas discrepancias proporcionan diferencias entre las predicciones del volumen de aire atrapado en la cámara de inyección, y provocan que el mínimo aire atrapado calculado con el modelo CFD se obtenga, normalmente, para aceleraciones ligeramente mayores que las predichas por el modelo basado en la aproximación de aguas poco profundas considerando los efectos de reflexión en la pared final de la cámara. También se ha encontrado una concordancia aceptable con resultados experimentales obtenidos para el parámetro de la aceleración del pistón, $\alpha$, que minimiza la cantidad de aire atrapado en una cámara de inyección de sección circular. Por otro lado, se ha comprobado que existe una concordancia aceptable entre los valores de aire atrapado predichos con el modelo numérico para la ley óptima de aceleración de la Ecuación (2.61) y las predicciones analíticas de aire atrapado nulo. 
Existe una ligera influencia del parámetro $L / H$ sobre los resultados numéricos de $A$ / ( $H L$ ) presentados en este capítulo, en especial para condiciones en las que pueden formarse chorros a lo largo del techo de la cámara que anticipen la rotura de la ola y el cierre de la entrada hacia la cavidad del molde, que no ha sido analizada en este trabajo, y que deberá ser objeto de estudio más detallado.

A pesar de los inconvenientes del modelo de aguas poco profundas respecto a las predicciones cuantitativas de la masa de aire atrapado, éste describe razonablemente bien la influencia de los parámetros de aceleración y de la fracción inicial de llenado en la masa de aire atrapada, por lo que se puede considerar como una herramienta sencilla y útil para elegir las condiciones de operación que reduzcan el aire atrapado a la vez que mantienen el tiempo de llenado tan bajo como sea requerido.

Se debería estudiar en más detalle los mecanismos de atrapamiento de aire que aparecen en la rotura de la ola y la interacción de ésta con las paredes de la cámara para determinar de una forma más precisa las variables óptimas del proceso. Sin embargo, se considera que el análisis del movimiento del metal fundido en la cámara de inyección que se ha presentado puede ser de gran ayuda para elegir apropiadamente las variables del proceso y minimizar la cantidad de aire atrapado durante el proceso de inyección. 


\section{Estudio de la evacuación de aire a través de los vientos}

En este capítulo se analiza la influencia de los efectos no estacionarios, que se han despreciado en modelos anteriores, en la evacuación del aire a través de los vientos en los procesos de fundición por inyección a presión. Se propone un modelo que considera al flujo de aire como unidimensional, adiabático y con fricción, y se analizan, para sistemas de evacuación atmosférica y al vacío, condiciones de evacuación para amplios rangos de los parámetros adimensionales relevantes. Para evacuación al vacío, donde el modelo de Bar-Meir et al. (1996) considera que en el viento existe bloqueo sónico durante todo el proceso de llenado, el modelo desarrollado se puede aplicar también para situaciones en las que no exista bloqueo sónico en el viento. El modelo se resuelve numéricamente mediante el método de las características y el método predictor-corrector de Euler modificado, y sus resultados se comparan con los obtenidos con modelos quasi-estacionarios propuestos por Bar-Meir et al. $(1996,1997)$. Se comprueba que pueden existir amplios rangos de condiciones de operación habituales para las que los efectos no estacionarios, despreciados en modelos anteriores, son importantes y se deben tener en cuenta para determinar la masa de aire atrapado al final del proceso de llenado. Se discutirá la elección de los parámetros de diseño y operación del sistema de evacuación que reducirían la cantidad de aire atrapado y de este modo la porosidad en las piezas fabricadas. 


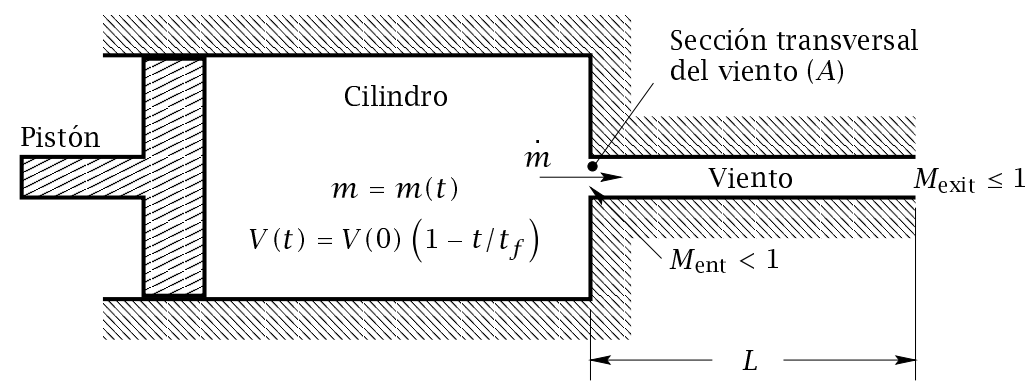

Figura 5.1: Modelo simplificado de evacuación de aire a través de los vientos (Bar-Meir et al., 1996, 1997).

\subsection{Modelo quasi-estacionario}

Bar-Meir et al. $(1996,1997)$ usaron un modelo simplificado, cuyo esquema se representa en la Figura 5.1, para determinar la evacuación de gas a través de los vientos. En este modelo, la parte de la cámara de inyección que inicialmente no está llena de metal líquido, el sistema de distribución y la cavidad del molde se consideran como parte de una única cavidad llamada cilindro, donde se supone que el gas está en reposo y que todas las magnitudes son uniformes en todo instante, y que el gas (principalmente aire) evoluciona isentrópicamente. Se considera que el pistón se mueve a velocidad constante.

Debido al periodo de tiempo tan pequeño en el que se produce el llenado del molde, Bar-Meir et al. (1996, 1997) consideraron que los fenómenos de transferencia de calor se pueden despreciar en todo el sistema. En el flujo unidimensional quasi-estacionario de un gas perfecto en un conducto de sección constante, la expresión (Shapiro, 1954)

$$
\frac{4 f L}{D}=\frac{1}{\gamma}\left(\frac{1}{M_{\mathrm{ent}}^{2}}-\frac{1}{M_{\mathrm{exit}}^{2}}\right)-\frac{\gamma+1}{2 \gamma} \ln \left(\frac{M_{\mathrm{exit}}^{2}}{M_{\mathrm{ent}}^{2}} \frac{(\gamma-1) M_{\mathrm{ent}}^{2}+2}{(\gamma-1) M_{\mathrm{exit}}^{2}+2}\right),
$$

donde $4 f L / D$ representa la resistencia en el viento*, $f$ es el valor promedio del coeficiente de fricción, $D$ es el diámetro hidráulico del viento, $M_{\text {ent }}$ es el número de Mach en la sección de entrada al viento y $M_{\text {exit }}$ es el número de Mach en la sección de salida del viento, permite calcular el valor de $4 f L / D$ necesario para conseguir una determinada variación del número de Mach a través del viento. Cuando existe bloqueo sónico $\left(M_{\text {exit }}=1\right)$, el número de Mach en la sección de entrada al viento alcanza

\footnotetext{
*En este capítulo, $L$ se utilizará para denotar la longitud equivalente del viento.
} 
su valor máximo $\left(M_{\mathrm{ent}}=M_{\max }\right)$, que dependerá del parámetro $4 f L / D$, como se deduce de la Ecuación (5.1). La relación entre la presión en la cavidad del molde (presión de remanso $p_{0}$ ) y la presión del aire en la salida del viento, $p_{\text {exit }}$, se puede expresar del siguiente modo (Shapiro, 1954):

$$
\left(\frac{p_{0}}{p_{\text {exit }}}\right)^{2}=\frac{M_{\text {exit }}^{2}}{M_{\text {ent }}^{2}}\left(1+\frac{\gamma-1}{2} M_{\text {exit }}^{2}\right)\left(1+\frac{\gamma-1}{2} M_{\text {ent }}^{2}\right)^{\frac{\gamma+1}{\gamma-1}} .
$$

La solución de las Ecuaciones (5.1) y (5.2) permite obtener, para un valor dado del parámetro $4 f L / D$ y de la relación $p_{0} / p_{\text {exit }}$, las propiedades del aire en el viento. Utilizando la ecuación de conservación de la masa en la cavidad del molde,

$$
\frac{\mathrm{d} m}{\mathrm{~d} t}+\dot{m}=0
$$

donde $\dot{m}$ representa el gasto másico de aire a través del viento, Bar-Meir et al. (1997) dedujeron la siguiente ecuación diferencial ordinaria para obtener la presión en la cavidad del molde en cada instante:

$$
\frac{\mathrm{d} \bar{p}}{\mathrm{~d} \bar{t}}=\frac{\gamma\left(1-\frac{t_{f}}{t_{c}} \bar{M} f\left[M_{\mathrm{ent}}(t)\right] \bar{p}^{\frac{\gamma-1}{2 \gamma}}\right)}{1-\bar{t}} \bar{p} ; \bar{p}(0)=1,
$$

donde $t_{f}$ es el tiempo de llenado del cilindro, $t_{c}$ es un tiempo característico de evacuación de aire definido por Bar-Meir et al. $(1996,1997)$ como $t_{c} \simeq m(0) / \dot{m}^{*}$, siendo $\dot{m}^{*}$ el gasto másico de aire a través del viento cuando existe bloqueo sónico y la presión y la temperatura en el cilindro permanecen en sus valores iniciales, y $\bar{p}, \bar{M}$ y $\bar{t}$ denotan las magnitudes adimensionales $p_{0}(t) / p_{0}(0), M_{\mathrm{ent}}(t) / M_{\max } \mathrm{y} t / t_{f}$, respectivamente, y $f\left[M_{\mathrm{ent}}(t)\right]$ es la función

$$
f\left[M_{\mathrm{ent}}(t)\right]=\left[1+\frac{\gamma-1}{2} M_{\mathrm{ent}}^{2}(t)\right]^{-(\gamma+1) /[2(\gamma-1)]} .
$$

Para el caso especial de evacuación al vacío, Bar-Meir et al. (1996) obtuvieron una ecuación simplificada suponiendo que el viento se bloquea instantáneamente en $t=0$ :

$$
\frac{\mathrm{d} \bar{p}}{\mathrm{~d} \bar{t}}=\frac{\gamma\left(1-\frac{t_{f}}{t_{c}} \bar{p}^{\frac{\gamma-1}{2 \gamma}}\right)}{1-\bar{t}} \bar{p} ; \bar{p}(0)=1 .
$$

Como se ha mencionado anteriormente, si $M_{\text {exit }}=1, M_{\mathrm{ent}}=M_{\max }$ sólo dependerá del parámetro $4 f L / D$; es decir, el modelo de Bar-Meir et al. (1996) para vacío no depende de la presión a la salida del viento (presión del depósito de vacío en este caso). Más adelante se resolverán las Ecuaciones (5.4) y (5.6) para comparar sus resultados con los del modelo no estacionario que a continuación se describe. 


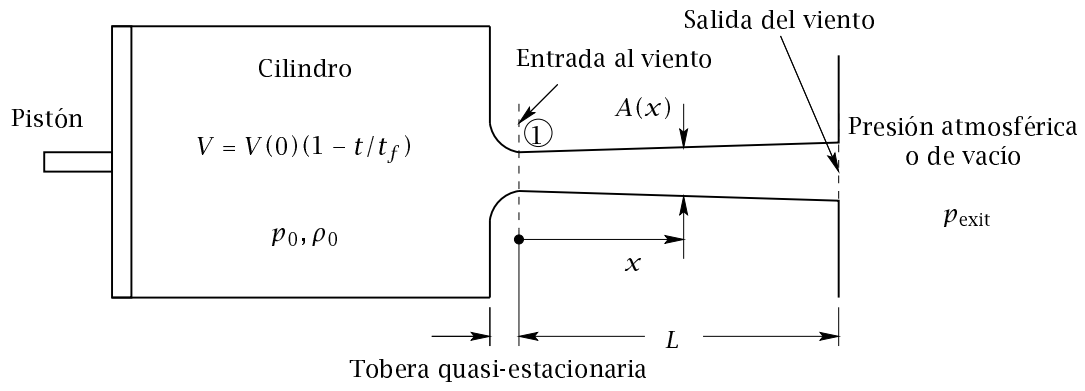

Figura 5.2: Esquema simplificado del sistema de evacuación de aire.

\subsection{Modelo no estacionario}

En la Figura 5.2 se representa el esquema del sistema de evacuación utilizado en el modelo no estacionario que se propone. Como la velocidad del pistón en la fase lenta es, normalmente, mucho menor que la de la fase rápida, la parte de la cámara de inyección que inicialmente no está llena de metal líquido no será incluida en el cilindro, a diferencia de lo que ocurre en el modelo de Bar-Meir et al. $(1996,1997)$. Se supondrá que el gas en el interior del cilindro está en reposo y que todas las magnitudes son uniformes en cada instante. Si se considera que el flujo de calor en las paredes del cilindro durante la fase rápida del proceso es despreciable, se puede demostrar fácilmente que el gas en el cilindro evoluciona isentrópicamente. Eligiendo un volumen de control que coincide con el del cilindro, $V$, la ecuación diferencial de la energía en forma no conservativa se puede expresar del siguiente modo $^{\dagger}$ :

$$
\frac{\mathrm{d} \mathcal{E}}{\mathrm{d} t}+p \frac{\mathrm{d} V}{\mathrm{~d} t}-\dot{Q}=0
$$

siendo $\mathcal{E}$ la energía interna del aire en el cilindro y $\dot{Q}$ el flujo de calor entrante a través de la sección 1 de entrada al viento. Suponiendo que el aire es térmicamente perfecto y que sus calores específicos $c_{v}$ y $c_{p}$ son constantes, se puede escribir que $\mathcal{E}=c_{v} m T$ y $\dot{Q}=c_{p} T \frac{\mathrm{d} m}{\mathrm{~d} t}$ en cada instante, donde $T$ es la temperatura del aire en el cilindro. Operando adecuadamente, la Ecuación (5.7) se puede expresar como:

$$
\frac{1}{\gamma-1} \frac{1}{T} \mathrm{~d} T+\frac{1}{V} \mathrm{~d} V-\frac{1}{m} \mathrm{~d} m=0,
$$

${ }^{\dagger}$ En este capítulo, $t$ representa el tiempo transcurrido desde el momento en el que el metal fundido llega a la sección de entrada a la cavidad del molde (inicio de la fase rápida de inyección). 
donde $\gamma=c_{p} / c_{v}$, e integrando la Ecuación (5.8) entre los estados 1 y 2, se obtiene la expresión

$$
\ln \left(\frac{T_{2}}{T_{1}}\right)^{1 /(\gamma-1)}=\ln \frac{V_{1}}{V_{2}}+\ln \frac{m_{2}}{m_{1}} .
$$

Operando nuevamente, esta expresión se puede reducir fácilmente a la conocida relación isentrópica entre la densidad $\rho$ y la presión $p$ de un gas perfecto

$$
\frac{p_{1}}{p_{2}}=\left(\frac{\rho_{1}}{\rho_{2}}\right)^{\gamma}
$$

En el modelo propuesto se supone que la velocidad del pistón es constante, y que el volumen de aire en el cilindro se reduce desde el valor inicial $V(0)$ hasta $V(t)=0$ en $t=t_{f}$. La densidad y la velocidad del sonido iniciales en el cilindro son, respectivamente, $\rho_{0}(0)$ y $c_{0}(0)$. Los vientos se representan como un conducto equivalente recto de longitud $L$ y diámetro hidráulico $D$, con una sección transversal de área $A$. El flujo en el conducto se considera adiabático con coeficiente de fricción constante $f$. La hipótesis de flujo adiabático se espera que sea apropiada debido al proceso de descarga tan rápido que se está considerando, en un tiempo demasiado pequeño para que los fenómenos de transferencia de calor puedan ser significativos. El flujo a través de la tobera en la sección de entrada al viento (Figura 5.2) se considera isentrópico y unidimensional. Se supone, además, que la longitud de la tobera es muy pequeña en relación a la longitud del viento $L$, por lo que el flujo en la tobera puede ser además considerado quasi-estacionario.

Las ecuaciones para flujo quasi-unidimensional no estacionario de un gas perfecto (Shapiro, 1954) se pueden expresar como

$$
\begin{gathered}
\frac{\partial \rho}{\partial t}+u \frac{\partial \rho}{\partial x}+\rho \frac{\partial u}{\partial x}+\rho u \frac{1}{A} \frac{\mathrm{d} A}{\mathrm{~d} x}=0, \\
\rho \frac{\partial u}{\partial t}+\rho u \frac{\partial u}{\partial x}+\frac{\partial p}{\partial x}=\mathcal{F}, \\
\frac{\partial p}{\partial t}+u \frac{\partial p}{\partial x}-c^{2}\left(\frac{\partial \rho}{\partial t}+u \frac{\partial \rho}{\partial x}\right)=(\gamma-1)(\rho q-u \mathcal{F}),
\end{gathered}
$$

donde $c=(p / \rho)^{1 / 2} \mathrm{y}$

$$
\mathcal{F}=-\frac{\rho u^{2}}{2} \frac{4 f}{D},
$$

$A=A(x)$ es el área de la sección transversal del conducto y $q=q(x, t)$ es la velocidad de transferencia de calor por unidad de flujo de masa. En este trabajo se considera $A=$ constante y $q=0$, y sólo se tienen en cuenta los efectos de cambio de área y transferencia de calor en la validación del código desarrollado para resolver las Ecuaciones (5.11), (5.12) y (5.13), 
comparando sus resultados con algunas soluciones analíticas obtenidas por Cai (1998) para las que se incluyen estos efectos, además de la fricción. Las variables dependientes en las Ecuaciones (5.11) a (5.13) son $\rho$, $p$ y $u$. Las condiciones iniciales son $\rho=\rho_{0}(0), p=p_{0}(0)$ y $u=0$ a lo largo de todo el viento en $t=0$. Si el flujo de aire es subsónico, la presión en la sección de salida del conducto $(x=L)$ se hace igual a $p_{0}(0)$ para evacuación atmosférica e igual a la presión del depósito de vacío para evacuación al vacío. Cuando el flujo de aire se invierte, las condiciones de contorno se deberán modificar apropiadamente.

La ley de conservación de masa en el cilindro de la Ecuación (5.3) se puede expresar del siguiente modo:

$$
\left(t_{f}-t\right) \frac{\mathrm{d} \rho_{0}}{\mathrm{~d} t}-\rho_{0}+\frac{A t_{f}}{V(0)} \rho_{1} u_{1}=0,
$$

donde el subíndice 1 denota las condiciones del gas en la sección final de la tobera (sección de entrada al viento).

La hipótesis de evolución isentrópica del aire en el cilindro proporciona una relación adicional obtenida a partir de la Ecuación (5.10):

$$
\frac{p_{0}(t)}{\rho_{0}(\mathrm{t})^{\gamma}}=\frac{p_{0}(0)}{\rho_{0}(0)^{\gamma}} \text {. }
$$

Las Ecuaciones (5.11) a (5.13) y (5.15) se pueden expresar adimensionalmente del siguiente modo:

$$
\begin{gathered}
\frac{L}{c_{0}(0) t_{f}} \frac{\partial \rho^{\prime}}{\partial t^{\prime}}+u^{\prime} \frac{\partial \rho^{\prime}}{\partial x^{\prime}}+\rho^{\prime} \frac{\partial u^{\prime}}{\partial x^{\prime}}+\rho^{\prime} u^{\prime} \frac{1}{A} \frac{\mathrm{d} A}{\mathrm{~d} x^{\prime}}=0, \\
\frac{L}{c_{0}(0) t_{f}} \rho^{\prime} \frac{\partial u^{\prime}}{\partial t^{\prime}}+\rho^{\prime} u^{\prime} \frac{\partial u^{\prime}}{\partial x^{\prime}}+\frac{\partial p^{\prime}}{\partial x^{\prime}}=-\frac{\rho^{\prime} u^{\prime 2}}{2} \frac{4 f L}{D}, \\
\frac{L}{c_{0}(0) t_{f}} \frac{\partial p^{\prime}}{\partial t^{\prime}}+u^{\prime} \frac{\partial p^{\prime}}{\partial x^{\prime}}-\gamma \frac{p^{\prime}}{\rho^{\prime}}\left(\frac{L}{c_{0}(0) t_{f}} \frac{\partial \rho^{\prime}}{\partial t^{\prime}}+u^{\prime} \frac{\partial \rho^{\prime}}{\partial x^{\prime}}\right) \\
=(\gamma-1) \rho^{\prime}\left(\frac{q L}{c_{0}^{3}(0)}+\frac{u^{\prime 3}}{2} \frac{4 f L}{D}\right), \\
\left(1-t^{\prime}\right) \frac{\mathrm{d} \rho_{0}^{\prime}}{\mathrm{d} t^{\prime}}-\rho_{0}^{\prime}+\frac{A}{\mathcal{A}} \rho_{1}^{\prime} u_{1}^{\prime}=0,
\end{gathered}
$$

y la relación de la Ecuación (5.16) resulta

$$
\frac{p_{0}^{\prime}}{\rho_{0}^{\prime \gamma}}=\frac{1}{\gamma}
$$

donde

$$
\mathcal{A}=\frac{V(0)}{c_{0}(0) t_{f}}
$$


y

$$
\rho^{\prime}=\frac{\rho}{\rho_{0}(0)}, \quad u^{\prime}=\frac{u}{c_{0}(0)}, \quad p^{\prime}=\frac{p}{\rho_{0}(0) c_{0}^{2}(0)}, \quad x^{\prime}=\frac{x}{L}, \quad t^{\prime}=\frac{t}{t_{f}} .
$$

De las Ecuaciones (5.17) a (5.20) y de las condiciones de contorno, para $A=$ constante y $q=0$, se deduce que la solución dependerá de ciertos números adimensionales formados por los parámetros característicos del problema $\left(c_{0}(0), \rho_{0}(0), p_{\text {exit }}, L, D, A, V(0), f, \gamma, t_{f}\right)$. Los números adimensionales, que se utilizan en este trabajo, son los que aparecen en las ecuaciones en forma adimensional anteriores y en las correspondientes condiciones de contorno adimensionalizas: $c_{0}(0) t_{f} / L, A / \mathcal{A}, 4 f L / D$, $p_{\text {exit }} / \rho_{0}(0) c_{0}^{2}(0)$ y $\gamma$.

\subsection{Resolución numérica del modelo no estacio- nario}

Para resolver numéricamente el sistema de Ecuaciones (5.11), (5.12) y (5.13), se ha utilizado el método de las características (Zucrow y Hoffman, 1977). Mediante este método, el sistema de ecuaciones anterior se puede sustituir por el siguiente sistema de ecuaciones características (véase Apéndice A.2):

$$
\begin{gathered}
\left(\frac{\mathrm{d} t}{\mathrm{~d} x}\right)_{\circ}=\lambda_{\circ}=\frac{1}{u} \text { (trayectorias de las partículas fluidas), } \\
\left(\frac{\mathrm{d} t}{\mathrm{~d} x}\right)_{ \pm}=\lambda_{ \pm}=\frac{1}{u \pm c} \text { (líneas de Mach), }
\end{gathered}
$$

y las correspondientes ecuaciones de compatibilidad:

$$
\mathrm{d} p-c^{2} \mathrm{~d} \rho=(\gamma-1)\left(\frac{\rho q}{u}-\mathcal{F}\right) \mathrm{d} x
$$

a lo largo de la trayectoria de las partículas fluidas, y

$$
\mathrm{d} p \pm \rho c \mathrm{~d} u=\left[-\frac{1}{A} \frac{\mathrm{d} A}{\mathrm{~d} x} \rho u c^{2} \pm c \mathcal{F}+(\gamma-1)(\rho q-u \mathcal{F})\right] \mathrm{d} t
$$

a lo largo de las líneas de Mach.

Las ecuaciones de compatibilidad anteriores especifican las condiciones que se deben satisfacer a lo largo de las correspondientes características. Los subíndices $\circ,+y-$ se emplean para denotar las características $C_{\circ}$, que representan las trayectorias de las partículas fluidas, las características positivas $C_{+}$y las características negativas $C_{-}$, respectivamente. Las curvas características representan los caminos a lo largo 

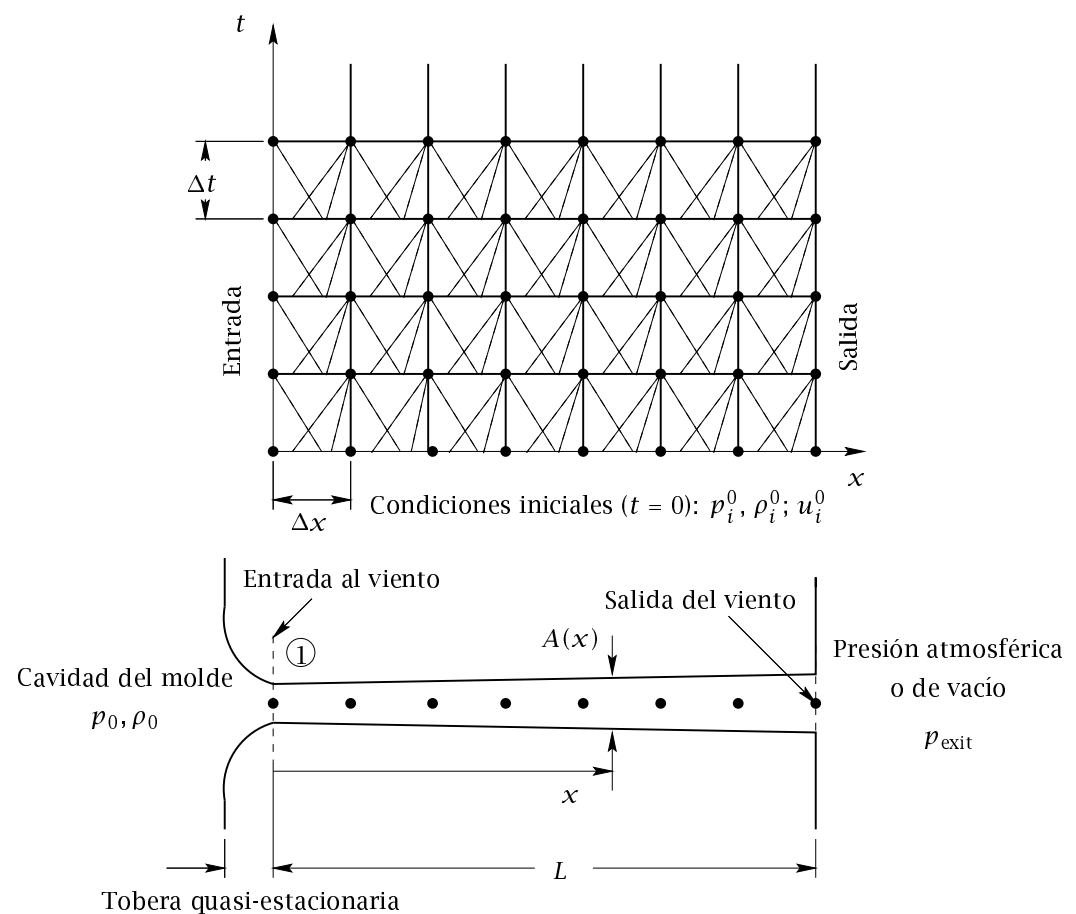

Figura 5.3: Esquema del viento y de la malla de diferencias finitas en el plano $x t$.

de los cuales la información se propaga a través del campo fluido. Por ejemplo, la velocidad de propagación a lo largo de las características $C_{+}$ y $C_{-}$se puede deducir de la Ecuación (5.25):

$$
\left(\frac{\mathrm{d} x}{\mathrm{~d} t}\right)_{ \pm}=\frac{1}{\lambda_{ \pm}}=u \pm c
$$

es decir, la velocidad de propagación a lo largo de ambas características relativa al fluido es igual a la velocidad del sonido. De igual forma, la velocidad de propagación a lo largo de la característica denotada por $C_{\text {。 }}$ se puede deducir de la Ecuación (5.24).

En la Figura 5.3 se representa el esquema de la malla de diferencias finitas en el plano $x t$ utilizada, en la que la localización de los puntos solución se especifica a priori ("Inverse Marching Method"). Las líneas características que pasan por cada punto solución se extienden hasta que cortan a la línea de tiempo constante que contiene los puntos solución del instante anterior. Las características entre el punto solución y los 
puntos de intersección (en adelante se llamarán puntos iniciales) se consideran líneas rectas. Las ecuaciones en diferencias finitas resultantes de la discretización de las Ecuaciones (5.24) a (5.27), que más adelante serán expuestas, han sido resueltas empleando el método predictor-corrector de Euler modificado. En el paso del predictor, los coeficientes de las ecuaciones en diferencias finitas se calculan en los puntos iniciales, donde las propiedades fluidas se determinan a partir de una interpolación natural cúbica spline basada en los puntos solución del instante anterior (Sección 5.3.3). En el paso del corrector, los coeficientes se calculan a partir de los valores promedio de las variables dependientes a lo largo de cada curva característica.

Las magnitudes del flujo en la sección de entrada al viento (sección 1) para flujo subsónico se pueden determinar en función de las propiedades de remanso, a partir de las expresiones que relacionan $\rho_{1} / \rho_{0}$ y $p_{1} / p_{0}$ con el número de Mach en la sección 1 para flujo isentrópico quasi-estacionario, y de la Ecuación (5.27) válida a lo largo de la característica negativa que procede del interior del viento. Las correspondientes ecuaciones discretizadas se han resuelto usando el método de Newton-Raphson. Para calcular las propiedades de remanso, la Ecuación (5.15) se ha resuelto mediante el método predictor-corrector de Euler modificado, usando el método explícito de Euler como predictor y una fórmula trapezoidal como corrector.

Para validar el código, los resultados numéricos se comparan con las soluciones analíticas obtenidas por Cai (1998). Por otro lado, también se comparan resultados numéricos para condiciones quasi-estacionarias con los obtenidos por Bar-Meir et al. $(1996,1997)$ para los casos de evacuación al vacío y evacuación atmosférica, respectivamente. A continuación se expondrán los detalles del procedimiento numérico para obtener las magnitudes del flujo en las secciones de entrada y salida y en el interior del viento.

\subsubsection{Discretización de las ecuaciones características y de compatibilidad}

Los puntos de la malla se sitúan igualmente espaciados a lo largo de los ejes $x$ y $t$, tal y como se representa en la Figura 5.3,

$$
\begin{aligned}
x_{i}=x_{0}+(i-1) \Delta x, & i=1,2, \ldots, I, \\
t_{j}=t_{0}+j \Delta t, & j=0,1, \ldots, J,
\end{aligned}
$$

donde $x_{0}$ corresponde a la coordenada $x$ del punto situado en la sección de entrada al viento $(i=1), I$ es el número de puntos de la malla en la dirección $x$ y $J$ es el número de intervalos de tiempo. El punto $i=0$ se utilizará para designar las propiedades del aire en el cilindro. Desde 
ahora, se escribirá $u_{i}^{j}$ para representar la velocidad $u\left(x_{i}, t_{j}\right)$, y de igual forma para las demás variables fluidas.

De la discretización de las Ecuaciones (5.24) y (5.26), resulta:

$$
\begin{gathered}
\Delta t=\lambda_{\circ} \Delta x, \\
\Delta p_{\circ}-B_{\circ} \Delta \rho_{\circ}=D_{\circ} \Delta x_{\circ},
\end{gathered}
$$

donde

$$
\begin{gathered}
\lambda_{\circ}=\frac{1}{u}, \\
D_{\circ}=(\gamma-1)\left(\frac{\rho q}{u}-\mathcal{F}\right), \\
B_{\circ}=c^{2},
\end{gathered}
$$

y de las Ecuaciones (5.25) y (5.27)

$$
\begin{gathered}
\Delta t=\lambda_{ \pm} \Delta x, \\
\Delta p \pm E_{ \pm} \Delta u=F_{ \pm} \Delta t,
\end{gathered}
$$

donde

$$
\begin{gathered}
\lambda_{ \pm}=\frac{1}{u \pm c}, \\
E_{ \pm}=\rho c, \\
F_{ \pm}=\left[-\frac{1}{A} \frac{\mathrm{d} A}{\mathrm{~d} x} \rho u c^{2} \pm c \mathcal{F}+(\gamma-1)(\rho q-u \mathcal{F})\right] .
\end{gathered}
$$

Para asegurar la estabilidad del procedimiento numérico se utiliza el criterio CFL de Courant, Friedrichs y Lewy (1928), con el que

$$
\Delta t_{\max }=\frac{\Delta x}{(u+c)_{\max }}
$$

donde $\Delta t_{\max }$ es el valor máximo del paso $\Delta t$ que asegura la estabilidad del procedimiento, siendo $(u+c)$ max el valor máximo de $u+c$ en cada instante. La experiencia demuestra que un valor apropiado para $\Delta t$ podría ser, aproximadamente, $0,5 \Delta t_{\max }$.

\subsubsection{Descripción del procedimiento numérico}

En las Figuras 5.4, 5.5 y 5.6 se representan los diagramas de flujo correspondientes a las subrutinas ENTRADA, INTERIOR y SALIDA desarrolladas para resolver el problema en los puntos situados en la sección de entrada, en el interior y en la sección de salida del viento, respectivamente. A continuación se expondrá detalladamente el procedimiento numérico empleado para resolver el problema en los puntos situados en el interior del viento. Más adelante se considerará el problema en las secciones de entrada y salida del viento, indicándose las condiciones de contorno impuestas en cada caso. 


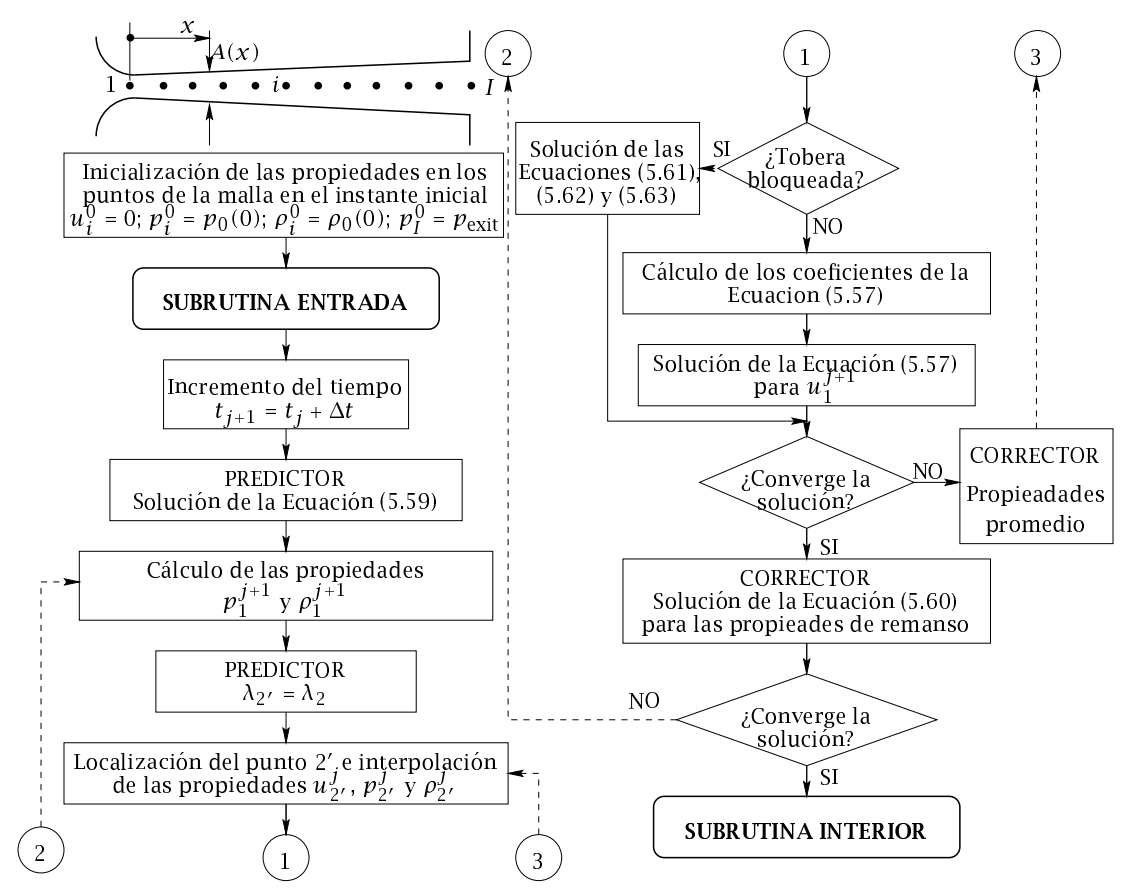

Figura 5.4: Diagrama de flujo para el cálculo de las propiedades del aire en la sección de entrada al viento. 


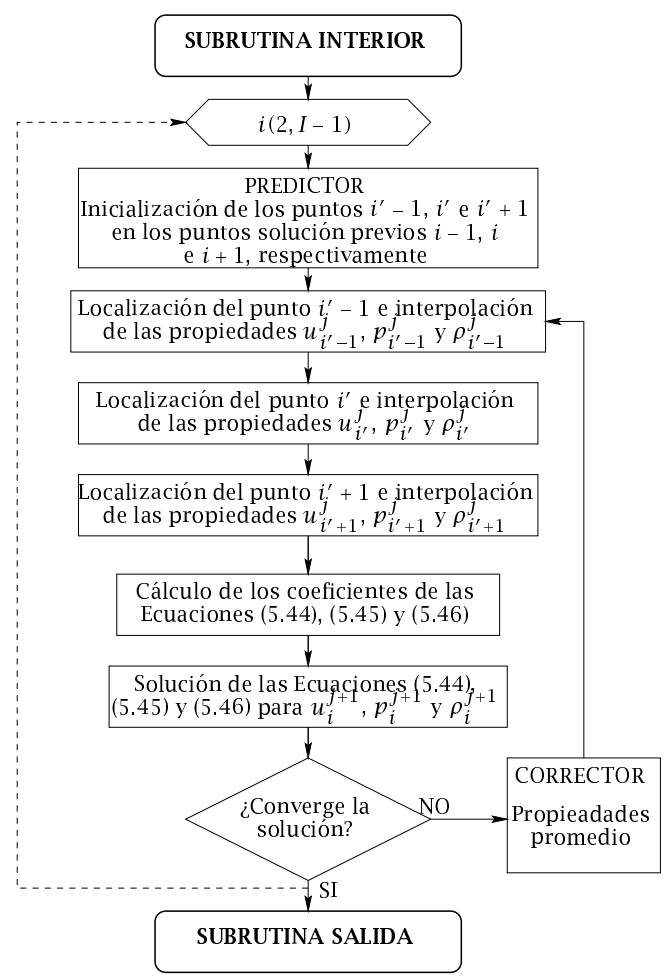

Figura 5.5: Diagrama de flujo para el cálculo de las propiedades del aire en el interior del viento. 


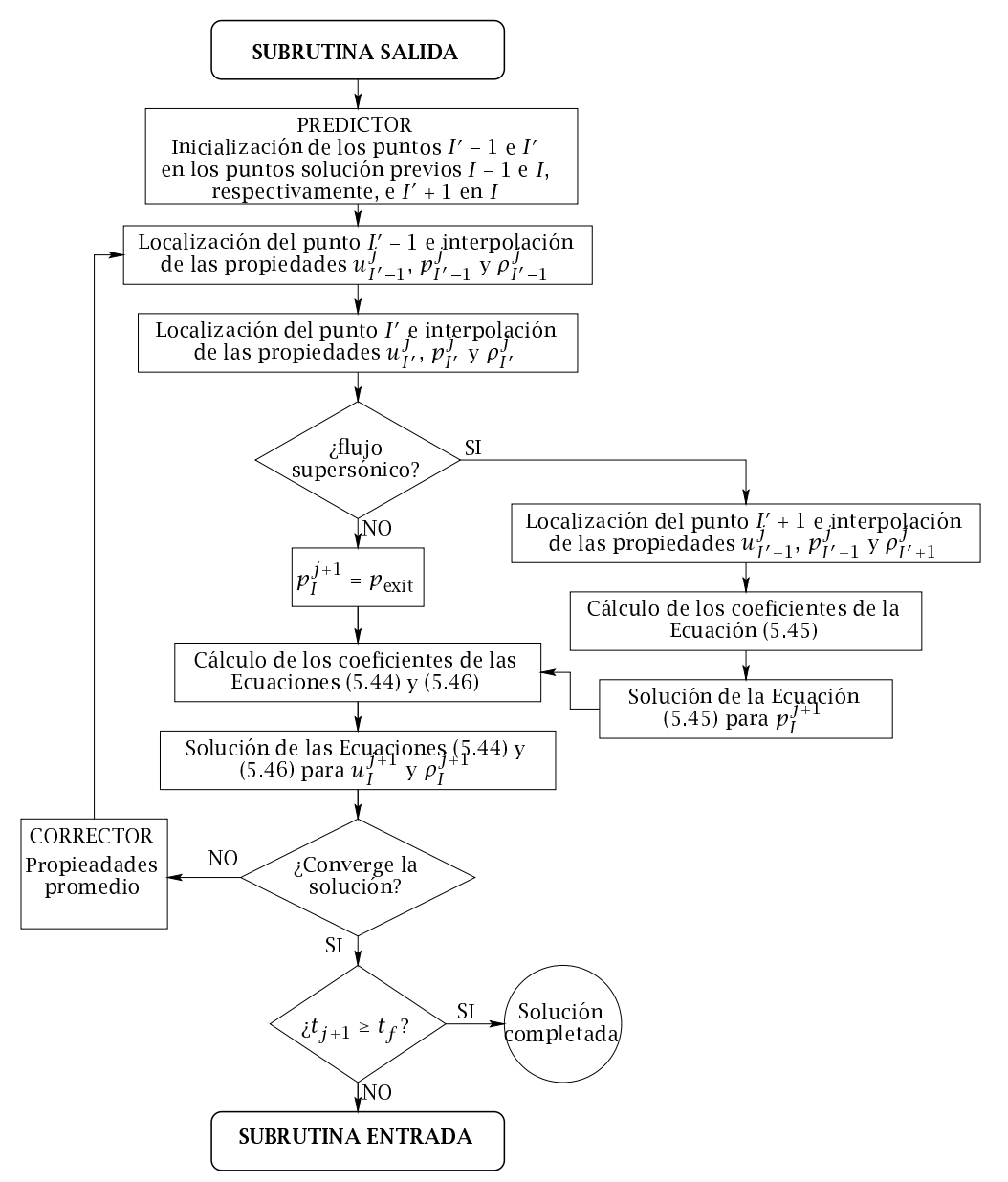

Figura 5.6: Diagrama de flujo para el cálculo de las propiedades del aire en la sección de salida del viento. 


\subsubsection{Interior del viento}

En la Figura 5.7 se representa la malla de diferencias finitas para un punto $i=2, \ldots, I-1$ localizado en el interior del viento (como se ha indicado anteriormente, el punto $i=1$ se encuentra situado en la sección de entrada al viento). En este caso, las ecuaciones características se pueden expresar en forma discretizada como:

$$
\begin{gathered}
\Delta t=\lambda_{+}\left(x_{i}^{j+1}-x_{i^{\prime}-1}^{j}\right), \\
\Delta t=\lambda_{-}\left(x_{i}^{j+1}-x_{i^{\prime}+1}^{j}\right), \\
\Delta t=\lambda_{\circ}\left(x_{i}^{j+1}-x_{i^{\prime}}^{j}\right),
\end{gathered}
$$

y las correspondientes ecuaciones de compatibilidad como:

$$
\begin{gathered}
p_{i}^{j+1}+E_{+} u_{i}^{j+1}=F_{+} \Delta t+p_{i^{\prime}-1}^{j}+E_{+} u_{i^{\prime}-1}^{j}, \\
p_{i}^{j+1}-E_{-} u_{i}^{j+1}=F_{-} \Delta t+p_{i^{\prime}+1}^{j}-E_{-} u_{i^{\prime}+1}^{j}, \\
p_{i}^{j+1}-B_{\circ} \rho_{i}^{j+1}=D_{\circ}\left(x_{i}^{j+1}-x_{i^{\prime}}^{j}\right)+p_{i^{\prime}}^{j}-B_{\circ} \rho_{i^{\prime}}^{j},
\end{gathered}
$$

donde el subíndice $i^{\prime}$ denota los puntos iniciales por los que pasan las características que llegan al punto solución y cuyas propiedades se obtendrán por interpolación a partir de los valores solución del instante $t_{j}$.

Predictor.- El predictor se inicia suponiendo que las características que llegan al punto solución son líneas rectas y que los puntos iniciales por los que pasan dichas características coinciden con los puntos solución del instante $t_{j}$, es decir, $x_{i^{\prime}-1}=x_{i-1}, x_{i^{\prime}}=x_{i}$ y $x_{i^{\prime}+1}=x_{i+1}$. De este modo, las pendientes de las características se pueden obtener sustituyendo las propiedades del flujo en dichos puntos en las Ecuaciones (5.32) y (5.37):

$$
\lambda_{+}^{P}=\lambda_{i^{\prime}-1}^{j}=\frac{1}{u_{i-1}^{j}+c_{i-1}^{j}} ; \lambda_{-}^{P}=\lambda_{i^{\prime}+1}^{j}=\frac{1}{u_{i+1}^{j}-c_{i+1}^{j}} ; \lambda_{\circ}^{P}=\lambda_{i^{\prime}}^{j}=\frac{1}{u_{i}^{j}} .
$$

La nueva localización de los puntos iniciales $\left(i^{\prime}-1, j\right),\left(i^{\prime}, j\right)$ e $\left(i^{\prime}+1, j\right)$ se puede obtener, fácilmente, sustituyendo los valores de las pendientes de la Ecuación (5.47) en las Ecuaciones (5.41), (5.42) y (5.43), respectivamente:

$$
\begin{aligned}
& x_{i^{\prime}-1}^{j}=x_{i}^{j+1}-\frac{\Delta t}{\lambda_{+}^{P}}, \\
& x_{i^{\prime}+1}^{j}=x_{i}^{j+1}-\frac{\Delta t}{\lambda_{-}^{P}},
\end{aligned}
$$




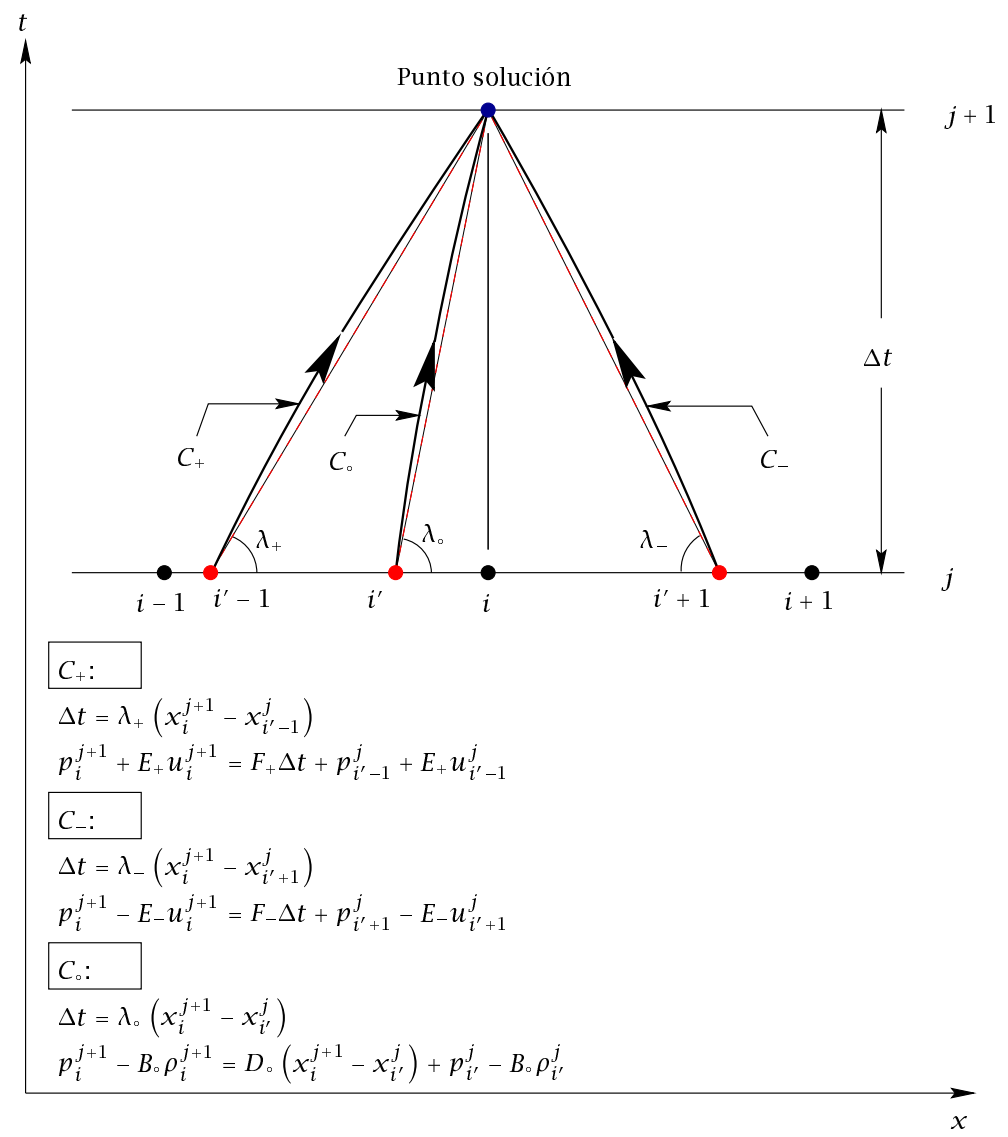

Figura 5.7: Malla de diferencias finitas en el plano $x t$ para un punto situado en el interior del viento. 


$$
x_{i^{\prime}}^{j}=x_{i}^{j+1}-\frac{\Delta t}{\lambda_{\circ}^{P}} .
$$

El superíndice $P$ se utiliza para denotar los valores obtenidos en la aplicación del predictor. Los valores de las variables fluidas $u, p$ y $\rho$ en estos nuevos puntos iniciales se obtendrán por interpolación a partir de los valores solución obtenidos en el instante anterior. Este procedimiento se repetirá iterativamente para mejorar los valores de las posiciones y propiedades del flujo en los puntos iniciales.

Suponiendo que las propiedades del flujo a lo largo de las características coinciden con las de los puntos iniciales que se acaban de calcular, se pueden determinar los coeficientes de las Ecuaciones (5.44), (5.45) y (5.46) para calcular las propiedades del flujo en el punto solución, completando de este modo la aplicación del predictor.

Corrector.- El corrector se inicia suponiendo que los valores de las propiedades del aire a lo largo de las características que llegan al punto solución son iguales a los valores promedio entre las propiedades del aire en los puntos iniciales $\left(i^{\prime}-1, j\right)$, $\left(i^{\prime}+1, j\right)$ e $\left(i^{\prime}, j\right)$, y las del punto solución $(i, j+1)$ obtenidas en la aplicación del predictor. De este modo, las propiedades corregidas de las tres características se podrán determinar del siguiente modo:

$$
\begin{array}{lll}
u_{\circ}=\frac{1}{2}\left(u_{i^{\prime}}^{j}+u_{i}^{j+1^{P}}\right) & u_{+}=\frac{1}{2}\left(u_{i^{\prime}-1}^{j}+u_{i}^{j+1^{P}}\right) & u_{-}=\frac{1}{2}\left(u_{i^{\prime}+1}^{j}+u_{i}^{j+1^{P}}\right) \\
p_{\circ}=\frac{1}{2}\left(p_{i^{\prime}}^{j}+p_{i}^{j+1^{P}}\right) & p_{+}=\frac{1}{2}\left(p_{i^{\prime}-1}^{j}+p_{i}^{j+1^{P}}\right) & p_{-}=\frac{1}{2}\left(p_{i^{\prime}+1}^{j}+p_{i}^{j+1^{P}}\right) \\
\rho_{\circ}=\frac{1}{2}\left(\rho_{i^{\prime}}^{j}+\rho_{i}^{j+1^{P}}\right) & \rho_{+}=\frac{1}{2}\left(\rho_{i^{\prime}-1}^{j}+\rho_{i}^{j+1^{P}}\right) & \rho_{-}=\frac{1}{2}\left(\rho_{i^{\prime}+1}^{j}+\rho_{i}^{j+1^{P}}\right)
\end{array}
$$

Con los valores promedio, y utilizando el mismo procedimiento descrito en la aplicación del predictor, se vuelven a calcular las pendientes de las líneas características, se corrigen las posiciones de los puntos iniciales y se obtienen, utilizando los valores promedio de la Ecuación (5.51), los coeficientes de las Ecuaciones (5.44), (5.45) y (5.46) para calcular las propiedades del flujo corregidas en el punto solución.

El corrector se aplicará iterativamente para mejorar la solución final, aunque se ha comprobado que, en general, se obtienen resultados suficientemente precisos con una o dos iteraciones.

\subsubsection{Sección de entrada al viento}

Si la tobera situada en la entrada al viento no está bloqueada $\left(M_{1}<1\right)$, dos de las tres propiedades del flujo $\left(u_{1}^{j+1}, p_{1}^{j+1} \circ \rho_{1}^{j+1}\right)$ se calculan a 
partir de la expresión que las relaciona con las propiedades del aire en el cilindro. Por ejemplo ${ }^{\ddagger}$ :

$$
\begin{aligned}
& p_{1}^{j+1}=p_{0}^{j+1}\left[1+(\gamma-1) \frac{\left(M_{1}^{j+1}\right)^{2}}{2}\right]^{\gamma /(1-\gamma)}, \\
& \rho_{1}^{j+1}=\rho_{0}^{j+1}\left[1+(\gamma-1) \frac{\left(M_{1}^{j+1}\right)^{2}}{2}\right]^{1 /(1-\gamma)},
\end{aligned}
$$

donde $M_{1}^{j+1}$ es el número de Mach en la sección 1 en el instante $t_{j+1}$. Expresando el número de Mach en función de la velocidad, y a partir de la relación isentrópica de la Ecuación (5.16), las Ecuaciones (5.52) y (5.53) se pueden expresar de la forma siguiente:

$$
\begin{aligned}
& p_{1}^{j+1}=p_{0}^{j+1}\left[1-\frac{\gamma-1}{2 \gamma} \frac{\rho_{0}^{j+1}}{p_{0}^{j+1}}\left(u_{1}^{j+1}\right)^{2}\right]^{\gamma /(\gamma-1)}, \\
& \rho_{1}^{j+1}=\rho_{0}^{j+1}\left[1-\frac{\gamma-1}{2 \gamma} \frac{\rho_{0}^{j+1}}{p_{0}^{j+1}}\left(u_{1}^{j+1}\right)^{2}\right]^{1 /(\gamma-1)} .
\end{aligned}
$$

La velocidad del aire $u_{1}^{j+1}$ se puede obtener a partir de la Ecuación (5.45) correspondiente a la ecuación de compatibilidad válida a lo largo de la característica negativa que llega a la sección de entrada desde el interior del viento (Figura 5.8):

$$
u_{1}^{j+1}=\frac{p_{1}^{j+1}-\left(F_{-} \Delta t+p_{2^{\prime}}^{j}-E_{-} u_{2^{\prime}}^{j}\right)}{E_{-}} .
$$

Introduciendo la Ecuación (5.54) en la Ecuación (5.56) se puede obtener la expresión que relaciona la velocidad del aire $u_{1}^{j+1}$ con las propiedades de remanso en el instante $t_{j+1}$ :

$$
u_{1}^{j+1}=\frac{p_{0}^{j+1}\left[1-\frac{\gamma-1}{2 \gamma} \frac{\rho_{0}^{j+1}}{p_{0}^{j+1}}\left(u_{1}^{j+1}\right)^{2}\right]^{\gamma /(\gamma-1)}-\left(F_{-} \Delta t+p_{2^{\prime}}^{j}-E_{-} u_{2^{\prime}}^{j}\right)}{E_{-}} .
$$

Esta ecuación se resolverá numéricamente empleando el método de Newton-Raphson. La localización del punto inicial 2', del que parte la característica negativa $C_{-}$que llega a la sección de entrada al viento en el

\footnotetext{
${ }^{\ddagger}$ Recuérdese que el flujo en la tobera se ha considerado isentrópico y unidimensional, y al ser ésta de longitud muy pequeña en relación a la del viento, el flujo en la tobera se puede considerar además quasi-estacionario.
} 
instante $t_{j+1}$, y los coeficientes $F_{-}$y $E_{-}$de la Ecuación (5.57) se calculan utilizando el método descrito en la sección anterior.

Para obtener las propiedades del aire en el cilindro se deben resolver simultáneamente las Ecuaciones (5.15) y (5.57). La Ecuación (5.15), la cual se puede expresar del siguiente modo:

$$
\frac{\mathrm{d} \rho_{0}}{\mathrm{~d} t}=f\left(t, \rho_{0}\right)=\frac{\rho_{0}}{t_{f}-t}-\frac{A}{V(0)\left(1-\frac{t}{t_{f}}\right)} \rho_{1} u_{1},
$$

se resuelve empleando el método predictor-corrector de Euler modificado, utilizando como predictor el método explícito de Euler

$$
\rho_{0}^{j+1^{P}}=\Delta t f\left(t_{j}, \rho_{0}^{j}\right),
$$

y como corrector la fórmula implícita del trapecio

$$
\rho_{0}^{j+1}=\frac{1}{2} \Delta t\left[f\left(t_{j}, \rho_{0}^{j}\right)+f\left(t_{j+1}, \rho_{0}^{j+1}{ }^{P}\right)\right] .
$$

El corrector se aplicará iterativamente para mejorar la precisión de la solución final.

Si la tobera está bloqueada $\left(M_{1}=1\right)$, teniendo en cuenta las Ecuaciones (5.52) y (5.53), la presión y la densidad en la sección de entrada al viento se pueden expresar de la forma siguiente:

$$
\begin{aligned}
& p_{1}^{j+1}=p_{0}^{j+1}\left(\frac{\gamma+1}{2}\right)^{\gamma /(1-\gamma)}, \\
& \rho_{1}^{j+1}=\rho_{0}^{j+1}\left(\frac{\gamma+1}{2}\right)^{1 /(1-\gamma)},
\end{aligned}
$$

y la velocidad será, obviamente, igual a la velocidad del sonido en dicha sección:

$$
u_{1}^{j+1}=\left(\frac{2 \gamma}{\gamma+1} \frac{p_{0}^{j+1}}{\rho_{0}^{j+1}}\right)^{1 / 2} .
$$

\subsubsection{Sección de salida del viento}

Si el flujo es subsónico, las propiedades del aire en la sección de salida del viento dependerán de las condiciones del aire exterior cerca de dicha sección. Por tanto, se debería estudiar el flujo de aire exterior en las proximidades de la sección de salida del viento. Sin embargo, es una aproximación razonable el que la presión en esta sección sea igual a la presión exterior $^{\S}$. En la Figura 5.9a se representa la malla de diferencias

\footnotetext{
${ }^{\S} p_{0}(0)$ en el caso de evacuación atmosférica, y la presión del depósito de vacío en el caso de evacuación al vacío.
} 


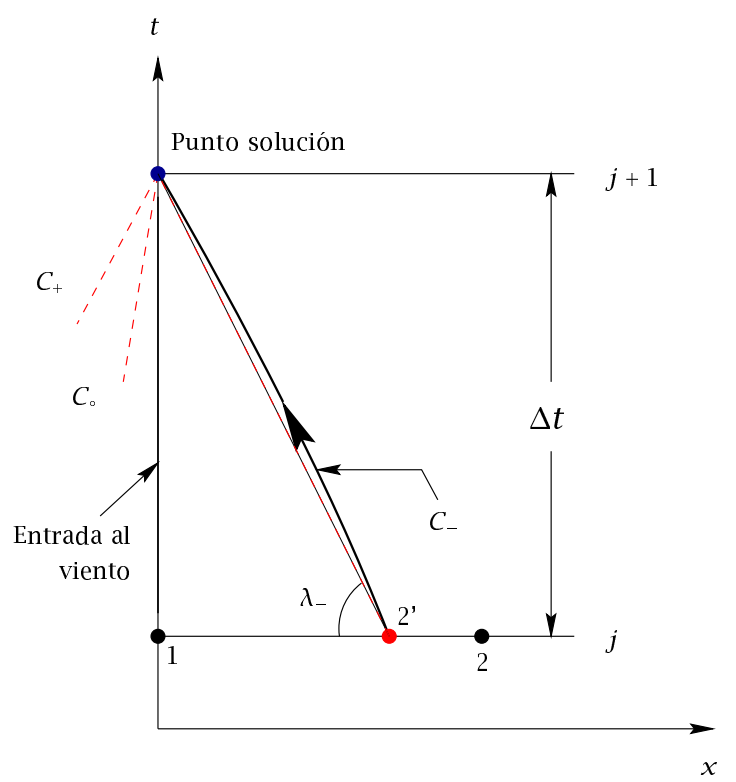

Figura 5.8: Malla de diferencias finitas en la sección de entrada al viento para condiciones de flujo subsónico. 
finitas empleada en este caso para condiciones de flujo subsónico. Las otras dos propiedades del aire, $u_{I}^{j+1}$ y $\rho_{I}^{j+1}$, se obtienen resolviendo las Ecuaciones (5.44) y (5.46) válidas a lo largo de las características $C_{+}$y $C_{\circ}$, respectivamente, que llegan a $x_{I}=L$ en el instante $t_{j+1}$. Para ello, se seguirá el procedimiento numérico descrito en las secciones anteriores.

Si el flujo es supersónico (Figura 5.9b), todas las propiedades del aire en la sección de salida del viento se obtienen resolviendo las Ecuaciones (5.44), (5.45) y (5.46) válidas a lo largo de las características $C_{+}, C_{-}$y $C_{\circ}$, respectivamente, que llegan a dicha sección en el instante $t_{j+1}$. Para ello, se utilizará el procedimiento numérico general descrito en la Sección 5.3.2.1 para un punto interior del viento. En este caso, obviamente, la solución del problema no dependerá de las propiedades del aire exterior en las proximidades de la sección de salida del viento.

\subsubsection{Procedimiento de interpolación de las propiedades en los puntos iniciales}

En éste y en el Capítulo 3 se han desarrollado sendos modelos numéricos para analizar el movimiento del metal fundido en la cámara de inyección y la evacuación del aire a través de los vientos, respectivamente. En ambos casos se ha utilizado una malla de diferencias finitas en la que la localización de los puntos solución se especifica a priori. Para obtener la solución del problema se deben resolver las ecuaciones de compatibilidad válidas a lo largo de las características que llegan a los puntos solución. Para ello, las propiedades en los puntos iniciales de los que parten dichas características se han obtenido por interpolación a partir de las propiedades en los puntos solución del instante anterior (Figura 5.10). Por ejemplo, la propiedad $y$ en el punto inicial $x$, comprendido entre los puntos solución previos $x_{i}$ y $x_{i+1}$, se podría haber obtenido interpolando linealmente a partir de las propiedades $y_{i}$ e $y_{i+1}$ en dichos puntos:

$$
y=A y_{i}+B y_{i+1},
$$

donde los valores de $A$ y $B$ son, respectivamente:

$$
\begin{aligned}
& A=\frac{x_{i+1}-x}{x_{i+1}-x_{i}}, \\
& B=1-A=\frac{x-x_{i}}{x_{i+1}-x_{i}} .
\end{aligned}
$$

En algunos casos, una interpolación de primer orden como ésta puede ser suficiente, pero se ha comprobado que en otros limita de forma importante la precisión numérica del procedimiento empleado. En esta tesis se ha utilizado una fórmula interpolatoria que tiene una derivada primera suave y una derivada segunda continua tanto dentro del intervalo como 

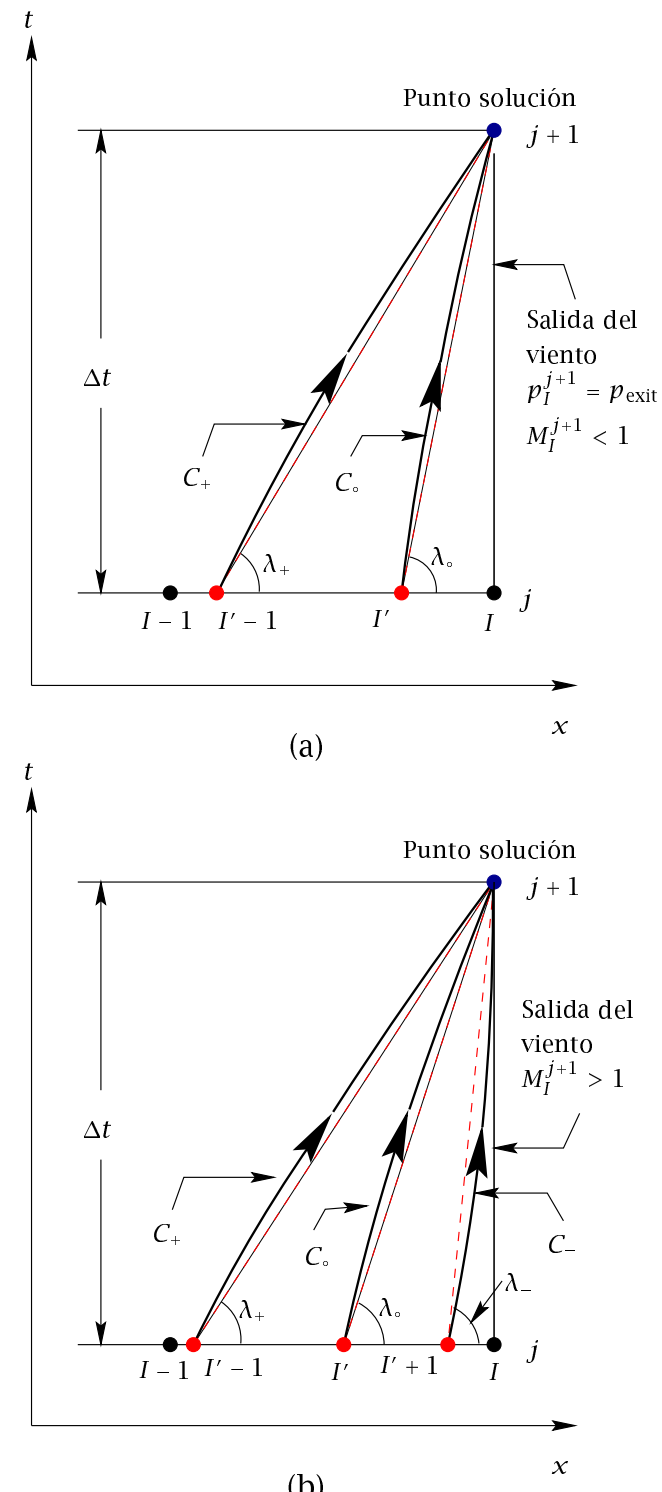

(b)

Figura 5.9: Malla de diferencias finitas en la sección de salida del viento. a) Flujo subsónico. b) Flujo supersónico. 


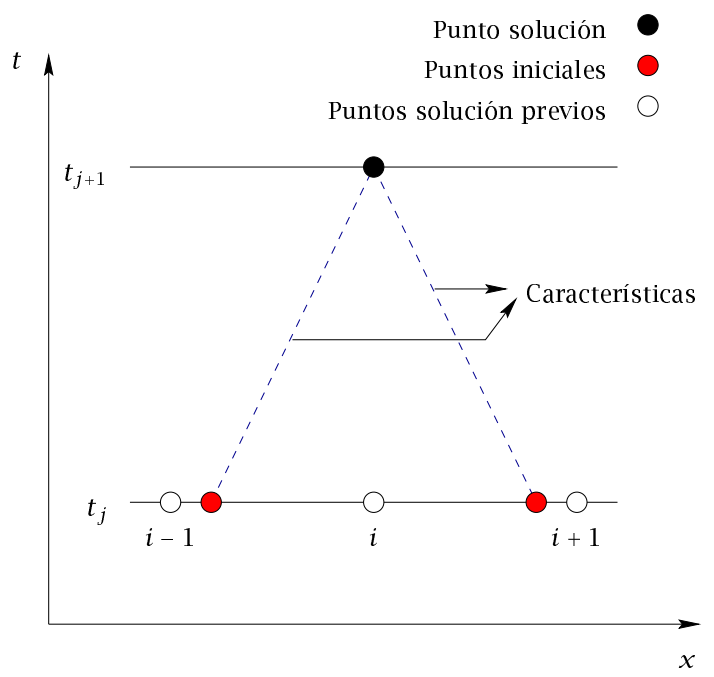

Figura 5.10: Malla de diferencias finitas.

en sus extremos. Dicha fórmula se obtiene añadiendo al segundo miembro de la Ecuación (5.64) un polinomio cúbico que se anula en $x_{i}$ y $x_{i+1}$, y cuya derivada segunda varía linealmente desde un valor $y_{i}^{\prime \prime}$ en $x_{i}$ hasta $y_{i+1}^{\prime \prime}$ en $x_{i+1}$ (véase, por ejemplo, Press et al. (1988)):

$$
y=A y_{i}+B y_{i+1}+C y_{i}^{\prime \prime}+D y_{i+1}^{\prime \prime},
$$

donde

$$
\begin{aligned}
& C=\frac{1}{6}\left(A^{3}-A\right)\left(x_{i+1}-x_{i}\right)^{2}, \\
& D=\frac{1}{6}\left(B^{3}-B\right)\left(x_{i+1}-x_{i}\right)^{2} .
\end{aligned}
$$

y $A$ y $B$ están dados en la Ecuación (5.65). (Puede comprobarse que $y^{\prime \prime}$ es la derivada segunda del mencionado polinomio.) Obsérvese que, para poder aplicar esta fórmula, se deben obtener las derivadas segundas en los puntos solución del instante anterior, lo que puede hacerse a partir de la condición de que la derivada primera, obtenida de la Ecuación (5.66), sea también continua en cualquier punto $x=x_{i}$. Igualando la derivada primera evaluada para $x=x_{i}$ en el intervalo $\left(x_{i-1}, x_{i}\right)$ con la derivada primera en el mismo punto evaluada en el intervalo $\left(x_{i}, x_{i+1}\right)$, y operando adecuadamente, se puede obtener la siguiente expresión para los puntos 
interiores $i=2, \ldots, I-1$ (Press et al., 1988):

$$
\frac{x_{i}-x_{i-1}}{6} y_{i-1}^{\prime \prime}+\frac{x_{i+1}-x_{i-1}}{3} y_{i}^{\prime \prime}+\frac{x_{i+1}-x_{i}}{6} y_{i+1}^{\prime \prime}=\frac{y_{i+1}-y_{i}}{x_{i+1}-x_{i}}-\frac{y_{i}-y_{i-1}}{x_{i}-x_{i-1}}
$$

lo que proporciona $I-2$ ecuaciones lineales para obtener los $I$ valores desconocidos $y_{i}^{\prime \prime}$ para $i=1, \ldots, I$. Para obtener una única solución de este sistema de ecuaciones se han impuesto las siguientes condiciones adicionales:

$$
\begin{aligned}
& y_{1}^{\prime \prime}=0, \\
& y_{I}^{\prime \prime}=0,
\end{aligned}
$$

en el primer y último punto de la malla, consiguiéndose de este modo un sistema de ecuaciones tridiagonal, lo que simplifica notablemente el cálculo de las derivadas segundas en los puntos solución del instante anterior. A este tipo de interpolación se le denomina "interpolación natural cúbica spline".

En la Figura 5.11 se presenta un ejemplo en el que se puede observar claramente que una interpolación de este tipo ofrece mejores resultados que una interpolación lineal. Sin embargo, existen situaciones en las que la aplicación de una interpolación natural cúbica spline puede no ser deseable. Para el mismo caso que el de la Figura 5.11 pero para un tiempo más pequeño, $t=0,1 t_{f}$, en la Figura 5.12 se aprecia que la velocidad del aire desde la mitad, aproximadamente, hasta la salida del viento aún es nula. Aunque, como se puede ver en esta figura, una fórmula de interpolación natural cúbica spline se acerca más a la solución obtenida con una malla refinada, existen zonas del viento, comprendidas entre $x / L=0,4$ y 0,5 , aproximadamente, para las que esta fórmula interpolatoria proporciona valores negativos de la velocidad del aire que podrán provocar problemas de convergencia numérica. Para evitar este problema, se ha empleado una interpolación lineal para instantes próximos al tiempo inicial y una interpolación natural cúbica spline cuando la velocidad del aire en la salida del viento deja de ser nula.

\subsubsection{Estudio de la precisión del procedimiento numérico}

Para condiciones de operación en las que los efectos no estacionarios son despreciables, los resultados numéricos del modelo desarrollado se han comparado con los obtenidos por Bar-Meir et al. (1996, 1997), habiéndose encontrado una excelente concordancia entre ellos (como se mostrará más adelante). Para comprobar la precisión del procedimiento numérico, se han comparado sus resultados con varias soluciones analíticas propuestas por Cai (1998).

En la Figura 5.13 se representa el error relativo $E=\left(u-u_{\text {exact }}\right) / u_{\text {exact }}$ entre las soluciones numéricas obtenidas utilizando diferentes tamaños 


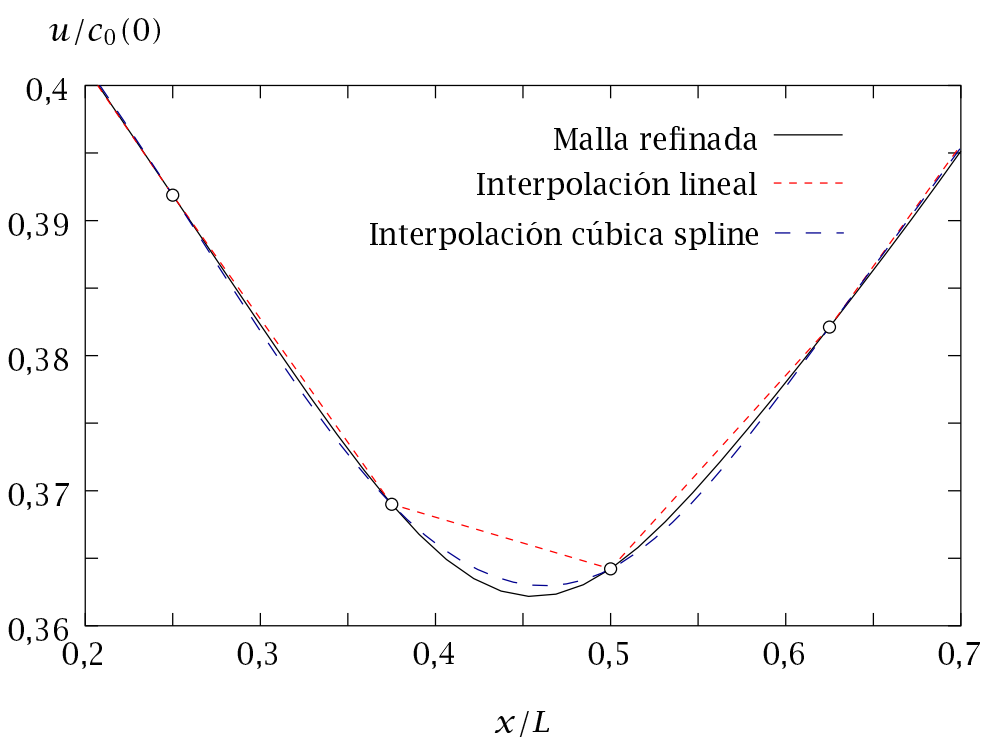

Figura 5.11: Resultados de una interpolación lineal y una interpolación natural cúbica spline para la velocidad adimensional del aire a lo largo del viento en $t=0,452 t_{f}$, para un caso con $4 f L / D=0, A / \mathcal{A}=0,735, c_{0}(0) t_{f} / L=3,673$ y $p_{\text {exit }}=$ $p_{0}(0)$. 


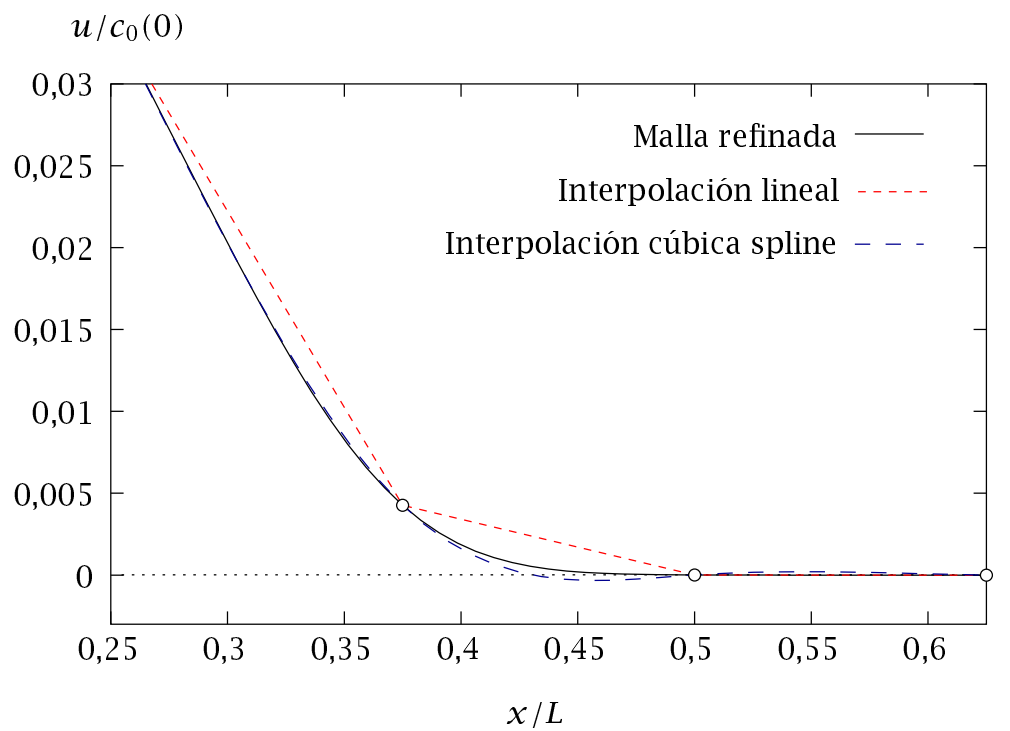

Figura 5.12: Resultados de una interpolación lineal y una interpolación natural cúbica spline para la velocidad adimensional del aire a lo largo del viento en $t=0,1 t_{f}$, para un caso con $4 f L / D=0, A / \mathcal{A}=0,735, c_{0}(0) t_{f} / L=3,673$ y $p_{\text {exit }}=$ $p_{0}(0)$. 

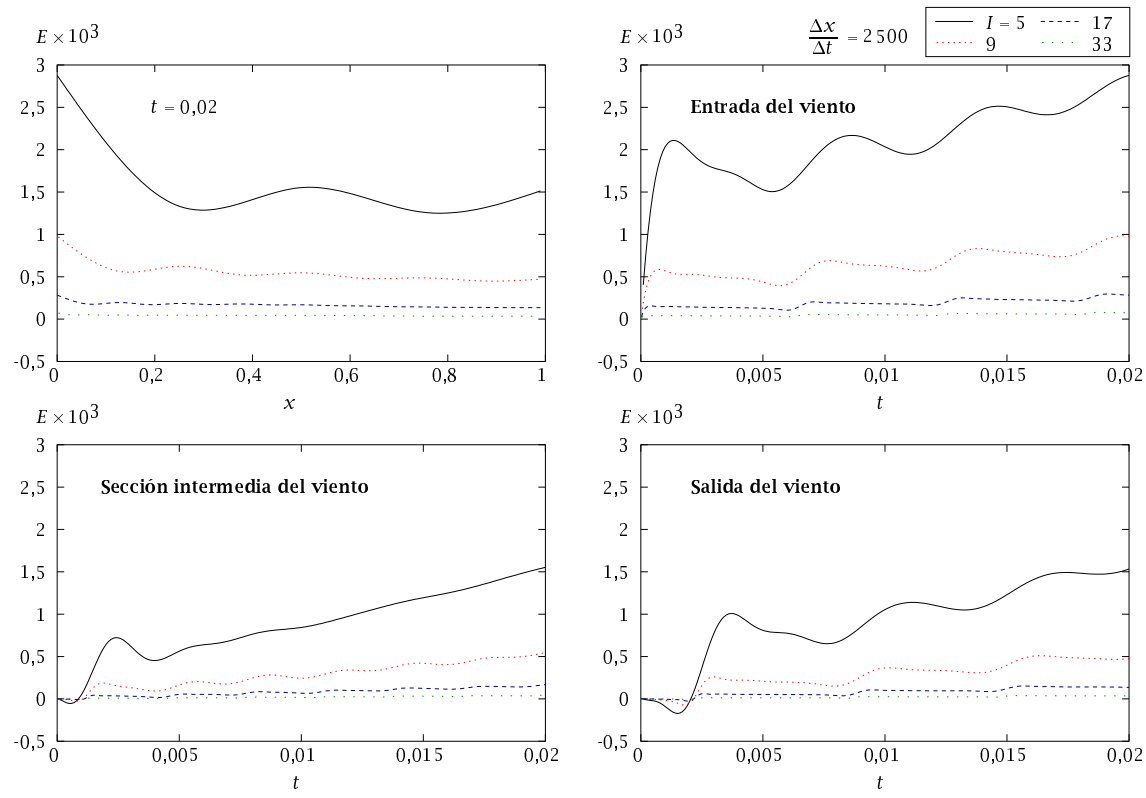

Figura 5.13: Error relativo entre la Ecuación (5.72) y las correspondientes soluciones numéricas para distintos tamaños de la malla, $\Delta x$ (diferentes valores de $I$ ), a lo largo del viento para $t=0,02$, y en las secciones de entrada, intermedia y de salida del viento en función de $t$. 
de malla, con $\Delta x / \Delta t=2500,{ }^{\natural 9}$ para el caso de flujo isentrópico con área de la sección transversal del viento variable, $A=1 /(x+1)$, y despreciando los efectos de fricción, y la siguiente solución analítica obtenida por Cai (1998):

$$
\begin{gathered}
p_{\text {exact }}=\text { constante }=p_{0}, \\
\rho_{\text {exact }}=\text { constante }=\rho_{0}, \\
u_{\text {exact }}=2(x+1) /(2 t+0,02),
\end{gathered}
$$

para $0 \leq t \leq 0,02$ y $0 \leq x \leq 1$. En el rango considerado, el flujo es subsónico, por lo que se han impuesto como condiciones de contorno en las secciones de entrada y salida del viento en todo instante los valores propuestos por Cai: $p_{1}=p_{0}, \rho_{1}=\rho_{0}$ y $p_{I}=p_{0}$, respectivamente. En la Figura 5.14 se representa el logaritmo del valor absoluto del error $E$ en función del logaritmo del tamaño de malla, $\Delta x$, en las secciones de entrada, intermedia y de salida del viento, para $t=0,02$. De esta figura se deduce, a partir de la pendiente de las curvas representadas, que el esquema numérico empleado tiene una precisión de segundo orden.

En la Figura 5.15 se representa el error relativo $E=\left(u-u_{\text {exact }}\right) / u_{\text {exact }}$ entre la solución numérica obtenida utilizando diferentes tamaños de malla para flujo de aire no isentrópico con coeficiente de fricción $f$, área de la sección transversal del viento constante $A$, y $q=-1 /\left(\Phi^{2} t^{3}\right)$, siendo $\Phi=f \sqrt{\pi / A}$, y la solución analítica encontrada por Cai (1998):

$$
\begin{gathered}
p_{\text {exact }}=\text { constante }=p_{0}, \\
\rho_{\text {exact }}=\text { constante }=\rho_{0}, \\
u_{\text {exact }}=1 /(\Phi t),
\end{gathered}
$$

para $0,02 \leq t \leq 0,04$ y $0 \leq x \leq 1$. Al igual que en el caso anterior, el flujo es subsónico en todo instante, por lo que se impondrán como condiciones de contorno en la sección de entrada al viento $p_{1}=p_{0} \mathrm{y}$ $\rho_{1}=\rho_{0}$, y en la sección de salida del viento $p_{I}=p_{0}$. El segundo orden de la precisión del esquema numérico se deduce en la Figura 5.15, en la que se ha representado el error $E$ en función del tamaño de la malla en las secciones de entrada, intermedia y de salida del viento, para $t=0,04$.

Para $0 \leq t \leq 0,02$ y $0 \leq x \leq 1$, y al igual que en la Figura 5.13, en la Figura 5.16 se representa el error relativo $E$ de la velocidad del flujo para otro caso no isentrópico en el que la densidad no es lineal en $x$ y la

\footnotetext{
"Esta relación se ha elegido para satisfacer la condición de estabilidad CFL. Todos los resultados representados en las Figuras 5.14, 5.15, 5.16 y 5.17 se han obtenido con esta misma relación.
} 


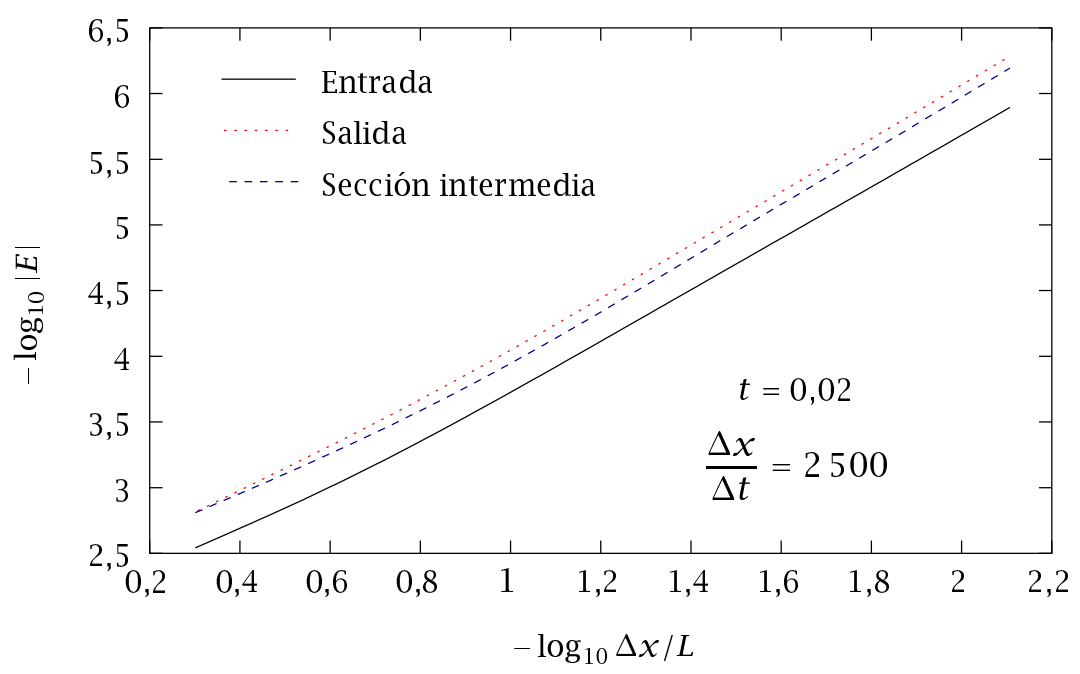

Figura 5.14: Error relativo entre la solución de la Ecuación (5.72) y la correspondiente solución numérica en función del tamaño de la malla, $\Delta x$, para $t=0,02$, en las secciones de entrada, intermedia y de salida del viento. 


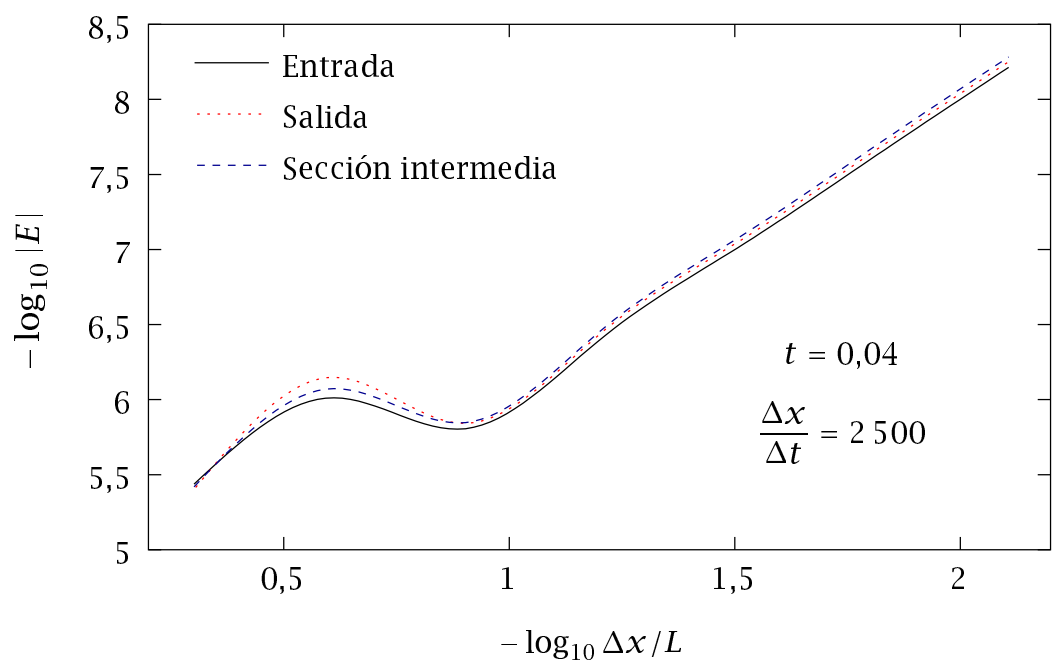

Figura 5.15: Error entre la Ecuación (5.75) y la correspondiente solución numérica en función del tamaño de la malla, $\Delta x$, para $t=0,04$, en las secciones de entrada, intermedia y de salida del viento. 
velocidad tampoco lo es en $x$ ni en $t$, con área del viento y coeficiente de fricción constantes, y

$$
q=-\frac{\gamma}{\gamma-1} p_{0} \frac{\Phi \exp ^{-\Phi x}\left(\Phi \exp ^{-\Phi x}+1\right)^{2}}{100(t+0,02)^{2} \exp ^{-\Phi x}}-\Phi\left[\frac{\exp ^{-\Phi x}+1 / \Phi}{t+0,02}\right]^{3},
$$

siendo $\Phi=f \sqrt{\pi / A}=0,2$, para el que Cai (1998) encontró la siguiente solución analítica:

$$
\begin{gathered}
p_{\text {exact }}=\text { constante }=p_{0}, \\
\rho_{\text {exact }}=\frac{100\left[(t+0,02) \exp ^{-\Phi x}\right]}{\left(\Phi \exp ^{-\Phi x}+1\right)^{2}}, \\
u_{\text {exact }}=\frac{\exp ^{-\Phi x}+1 / \Phi}{t+0,02} .
\end{gathered}
$$

En la Figura 5.17 se comparan los resultados del error relativo $E$ entre la Ecuación (5.79) y las correspondientes soluciones numéricas obtenidas con distintos tamaños de la malla para $t=0,02$, en las secciones de entrada, intermedia y de salida del viento, utilizando una interpolación natural cúbica spline y los obtenidos mediante una interpolación lineal. Se puede observar que la precisión de los resultados obtenidos mediante una interpolación spline es siempre mayor que la obtenida empleando una interpolación lineal. Además, en la Figura 5.17 se observa que con una fórmula interpolatoria del tipo spline la precisión del esquema numérico es de segundo orden, mientras que con una interpolación lineal, para casos no lineales en $x$ como éste, no se consigue alcanzar el orden de convergencia formal del procedimiento numérico empleado.

\subsubsection{Otros aspectos numéricos}

Como ya se ha indicado, todos los resultados presentados se han obtenido utilizando el método predictor-corrector de Euler modificado. En esta sección, y a modo de ejemplo, se comparan estos resultados para las propiedades del aire en el cilindro con los obtenidos sustituyendo dicho método, exclusivamente para resolver la Ecuación (5.15), en primer lugar, por el método de Runge-Kutta de cuarto orden:

$$
\begin{aligned}
k_{1} & =\Delta t f\left(t_{j}, \rho_{0}^{j}\right) \\
k_{2} & =\Delta t f\left(t_{j}+\frac{\Delta t}{2}, \rho_{0}^{j}+\frac{k_{1}}{2}\right) \\
k_{3} & =\Delta t f\left(t_{j}+\frac{\Delta t}{2}, \rho_{0}^{j}+\frac{k_{2}}{2}\right) \\
k_{4} & =\Delta t f\left(t_{j}+\Delta t, \rho_{0}^{j}+k_{3}\right) \\
\rho_{0}^{j+1} & =\rho_{0}^{j}+\frac{k_{1}}{6}+\frac{k_{2}}{3}+\frac{k_{3}}{3}+\frac{k_{4}}{6},
\end{aligned}
$$



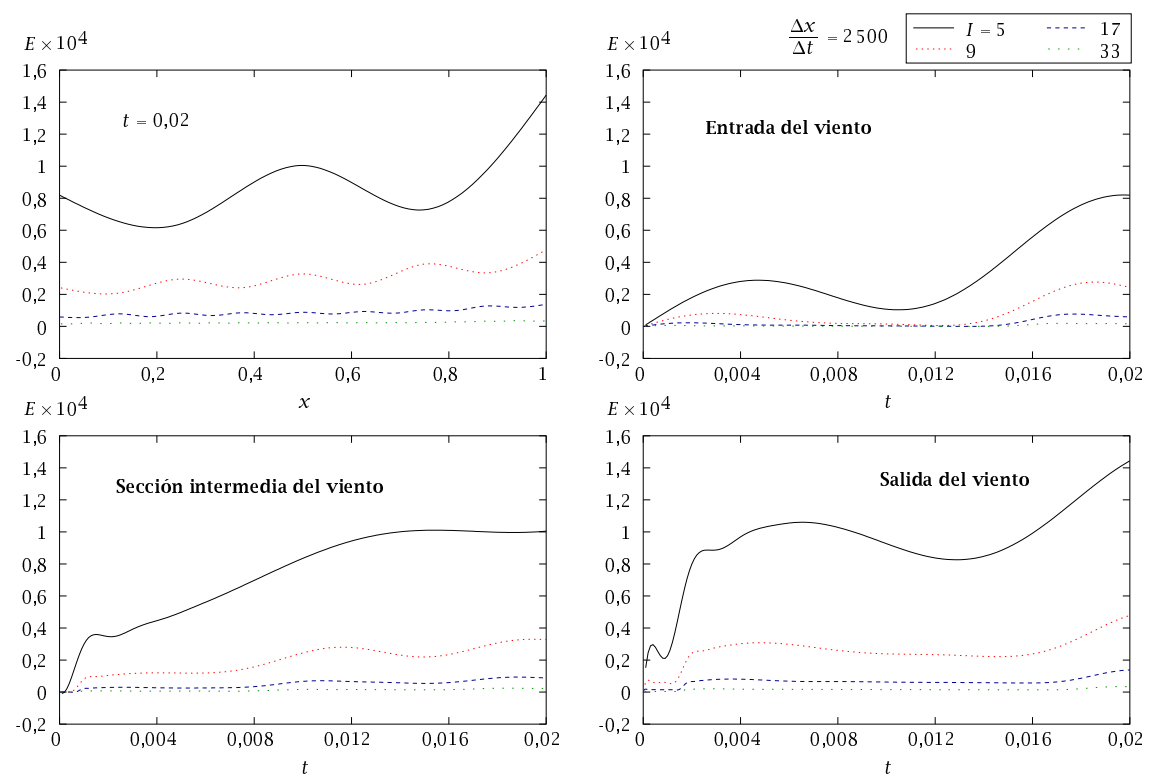

Figura 5.16: Error relativo entre la Ecuación (5.79) y las correspondientes soluciones numéricas obtenidas con distintos tamaños de la malla (diferentes valores de $I$ ), para $t=0,02$ a lo largo del viento, y en las secciones de entrada, intermedia y de salida del viento en función de $t$. 


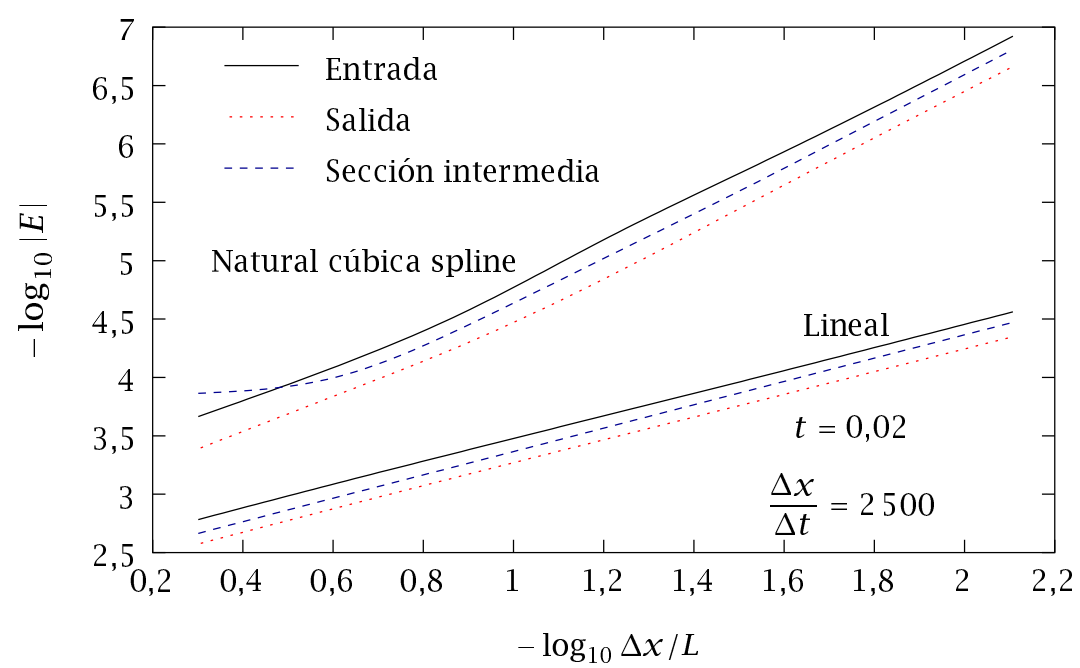

Figura 5.17: Error relativo entre la Ecuación (5.79) y la correspondiente solución numérica en función del tamaño de la malla, $\Delta x$, para $t=0,02$, en las secciones de entrada, intermedia y de salida del viento, utilizando una interpolación natural cúbica spline y una interpolación lineal. 
y, en segundo lugar, por el método predictor-corrector de Adams-BashforthMoulton, en el que se emplea como predictor la fórmula explícita de Adams-Bashforth de cuarto orden

$$
\begin{aligned}
\rho_{0}^{j+1^{P}}=\frac{\Delta t}{24}\left[55 f\left(t_{j}, \rho_{0}^{j}\right)\right. & -59 f\left(t_{j-1}, \rho_{0}^{j-1}\right) \\
& \left.+37 f\left(t_{j-2}, \rho_{0}^{j-2}\right)-9 f\left(t_{j-3}, \rho_{0}^{j-3}\right)\right],
\end{aligned}
$$

y como corrector la fórmula implícita de Adams-Moulton, también de cuarto orden

$$
\begin{aligned}
\rho_{0}^{j+1^{C}}=\frac{\Delta t}{24}\left[9 f\left(t_{j+1}, \rho_{0}^{j+1^{P}}\right)\right. & +19 f\left(t_{j}, \rho_{0}^{j}\right) \\
& \left.-5 f\left(t_{j-1}, \rho_{0}^{j-1}\right)+f\left(t_{j-2}, \rho_{0}^{j-2}\right)\right] .
\end{aligned}
$$

En la Figura 5.18 se representa la presión adimensional del aire en el cilindro para condiciones de evacuación atmosférica en función del tiempo, utilizando los métodos predictor-corrector de Euler modificado y de Adams-Bashforth-Moulton, y el método de Runge-Kutta para distintos tamaños de malla con, $A / \mathcal{A}=0,1, c_{0}(0) t_{f} / L=10$ y $4 f L / D=5$. Todos los resultados de la Figura 5.18 han sido obtenidos con la relación $t_{f} \Delta x /(L \Delta t)=15,62$. Se puede observar que el método de Runge-Kutta, incluso para mallas con un número de nodos $I$ a lo largo de la coordenada $x$ tres veces superior a los utilizados con los métodos predictor-corrector considerados, ofrece claramente resultados menos precisos. Además se observa que el método de Adams-Bashforth-Moulton, de orden superior al de Euler modificado, no mejora prácticamente los resultados obtenidos.

\subsection{Discusión de resultados de los modelos de evacuación de aire}

\subsubsection{Evacuación atmosférica}

En las Figuras 5.19 y 5.20 se comparan, para condiciones de evacuación atmosférica, los resultados para la presión en el cilindro en función del tiempo obtenidos con el modelo no estacionario presentado en este capítulo y los obtenidos con el modelo quasi-estacionario de Bar-Meir et al. (1997). La Figura 5.19 se ha obtenido despreciando los efectos de fricción, y la Figura 5.20 para un parámetro $4 f L / D$ típico, igual a 5 . El parámetro $c_{0}(0) t_{f} / L$ varía desde un valor igual a 5 en las Figuras $5.19 \mathrm{a}$ y 5.20 a hasta 50 en las Figuras 5.19 b y 5.20 b.

En las Figuras 5.19 y 5.20 se puede observar que los efectos no estacionarios aumentan conforme los parámetros $4 f L / D$ y $c_{0}(0) t_{f} / L$ disminuyen y, como cabía esperar, son despreciables para valores pequeños de la 


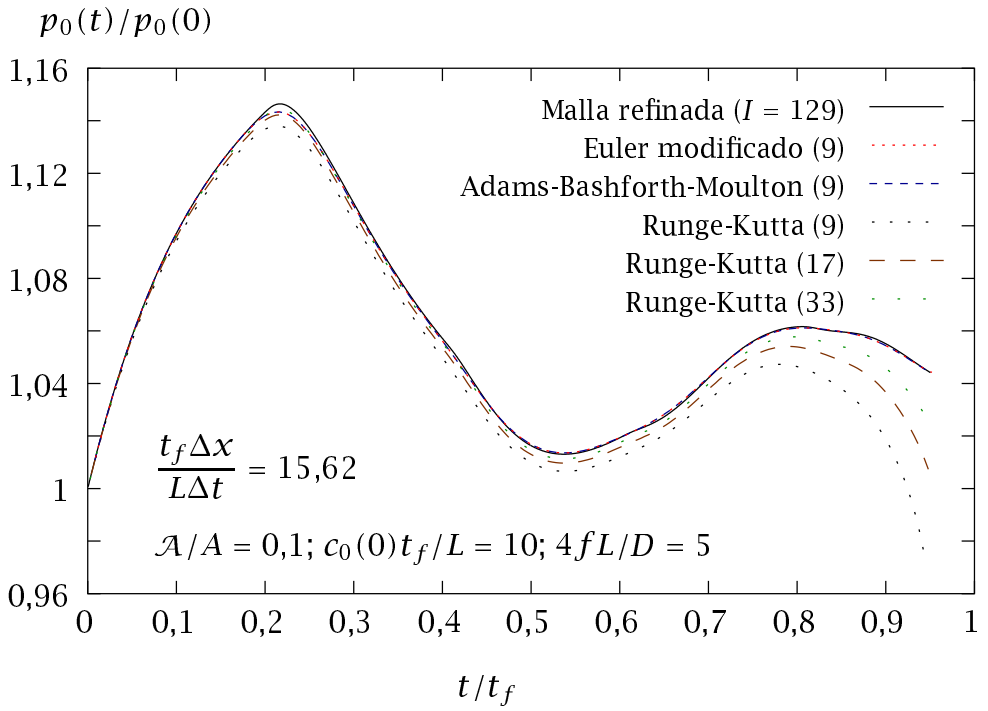

Figura 5.18: Presión adimensional del aire en el cilindro para condiciones de evacuación atmosférica en función del tiempo, utilizando los métodos predictor-corrector de Euler modificado y de Adams-Bashforth-Moulton, y un método de RungeKutta para distintos tamaños de malla, con $A / \mathcal{A}=0,1$, $c_{0}(0) t_{f} / L=10$ y $4 f L / D=5$. 

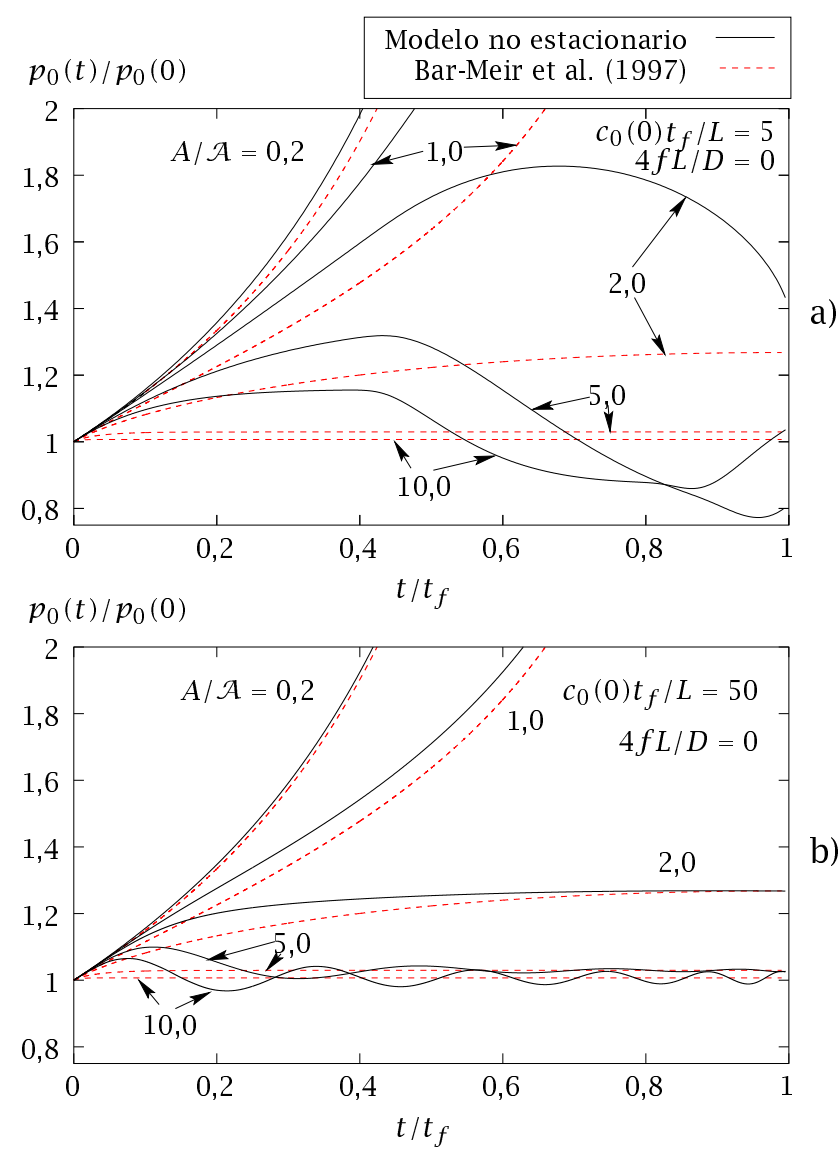

Figura 5.19: Relación adimensional de la presión en el cilindro en función del tiempo adimensional para evacuación atmosférica, $4 f L / D=0$ y diferentes valores de $A / \mathcal{A}$. a) $c_{0}(0) t_{f} / L=5$. b) $c_{0}(0) t_{f} / L=50$. 

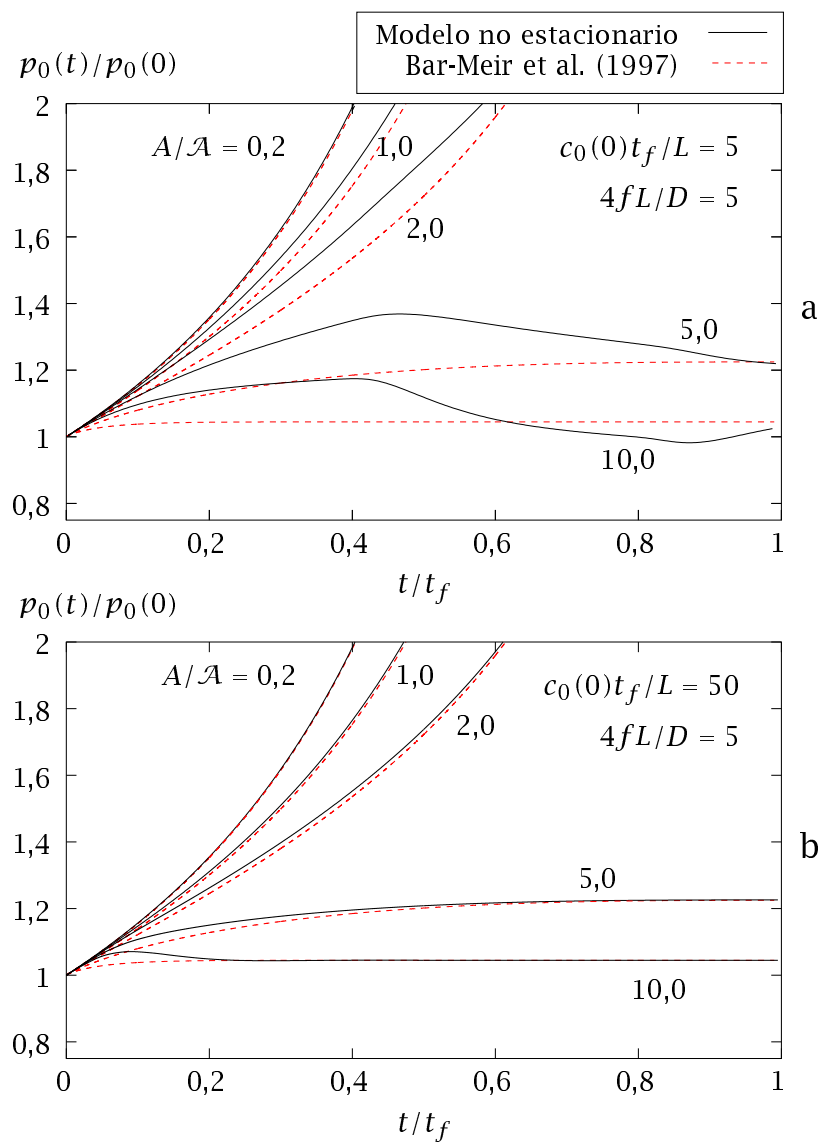

Figura 5.20: Relación adimensional de la presión en el cilindro en función del tiempo adimensional para evacuación atmosférica, $4 f L / D=5$ y diferentes valores de $A / \mathcal{A}$. a) $c_{0}(0) t_{f} / L=5$. b) $c_{0}(0) t_{f} / L=50$. 
relación $A / \mathcal{A}$. Para valores más grandes de $A / \mathcal{A}$, los efectos no estacionarios provocan inicialmente un aumento más rápido de la presión en el cilindro que el correspondiente al modelo quasi-estacionario, y para valores suficientemente grandes de $A / \mathcal{A}$ la presión en el cilindro oscila alrededor de la curva obtenida con el modelo quasi-estacionario. La frecuencia de estas oscilaciones aumenta conforme aumentan los parámetros $A / \mathcal{A}$ y $c_{0}(0) t_{f} / L$. Comparando las Figuras 5.19 y 5.20 , se puede observar también que los efectos de fricción producen un aumento apreciablemente mayor de la presión en el cilindro para los dos casos, quasi-estacionario y no estacionario, y una atenuación de los efectos no estacionarios. Los valores típicos del parámetro de fricción $4 f L / D$ pueden estar entre 0,5 y 50 (Bar-Meir et al., 1997), y el parámetro $c_{0}(0) t_{f} / L$ suele variar, normalmente, entre 5 y 50 , por lo que los efectos no estacionarios pueden ser importantes en situaciones habituales de operación, en las que deben ser tenidos en cuenta para conseguir un diseño apropiado de los sistemas de evacuación.

Bar-Meir et al. (1996) mostraron que, cuando el flujo está bloqueado durante todo el proceso de llenado, como suponen en su modelo quasiestacionario para evacuación al vacío, el siguiente valor del área de la sección del viento hace que la presión en el cilindro permanezca constante en todo instante:

$$
A_{c}=\frac{\mathcal{A}}{M_{\max }\left(1+\frac{\gamma-1}{2} M_{\max }^{2}\right)^{\frac{\gamma+1}{2(1-\gamma)}}},
$$

donde $M_{\max }$ es el número de Mach máximo en la sección de entrada al viento, cuyo valor puede obtenerse de la Ecuación (5.1) haciendo $M_{\text {exit }}=$ 1. Bajo estas condiciones de bloqueo sónico, también mostraron que $p_{0}(t) / p_{0}(0)$ sólo depende de $A / A_{c}$. Para evacuación atmosférica y condiciones quasi-estacionarias, Bar-Meir et al. (1997) han estudiado los efectos de $A / A_{c}$ en la evolución de la presión y la masa de aire en el cilindro, considerando que la influencia del parámetro $4 f L / D$ es bastante pequeña. Esta aproximación es más adecuada para valores suficientemente grandes o pequeños de $A / A_{c}$, para los que $p_{0}(t) / p_{0}(0)$ solamente depende de $A / A_{\mathcal{C}}$, mientras que para valores intermedios de $A / A_{\mathcal{C}}$, en torno a uno, $p_{0}(t) / p_{0}(0)$ también depende de $4 f L / D$, como se puede ver en la Figura 5.21. En este trabajo se ha usado $\mathcal{A}$, en lugar de $A_{c}$, para adimensionalizar el área del viento y mostrar explícitamente la influencia del parámetro de fricción, del que $A_{c}$ depende a través de $M_{\max }$ (Ecuación (5.83)).

Para condiciones de evacuación atmosférica y con valores de $4 \mathrm{fL} / \mathrm{D}=$ 0,5 y 50 , en las Figuras 5.22a, 5.22b y 5.22c, respectivamente, se representa la fracción de masa de aire restante en el cilindro en función del área adimensional del viento $A / \mathcal{A}$, para diferentes tiempos adimensionales, $t / t_{f}$, y distintos valores del parámetro $c_{0}(0) t_{f} / L$. Se puede observar que 


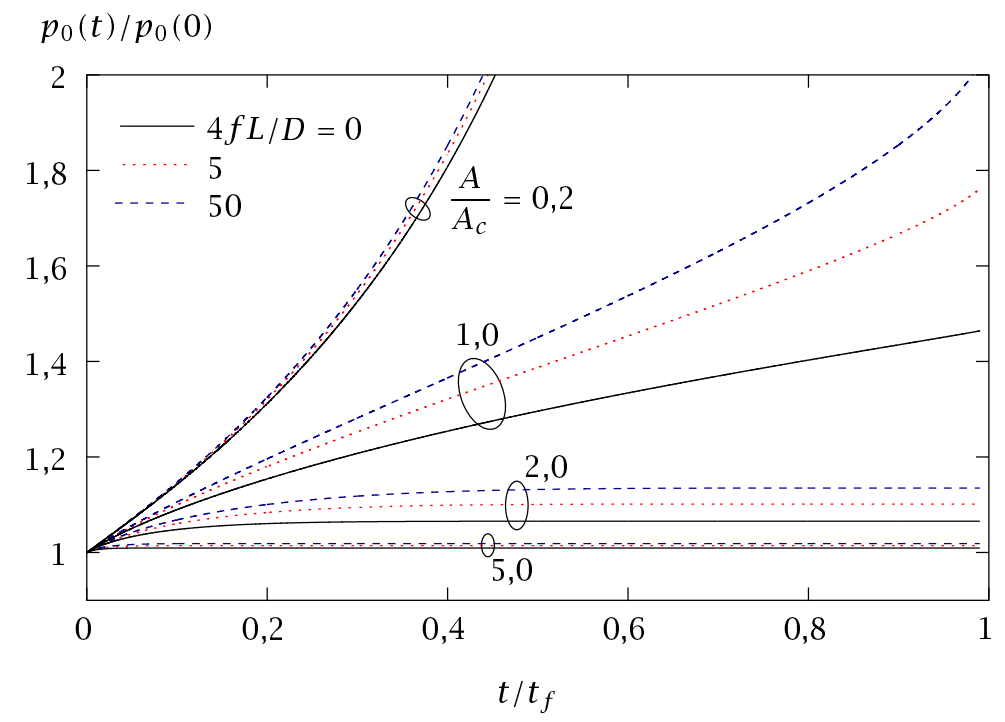

Figura 5.21: Relación adimensional de la presión en el cilindro en función del tiempo adimensional para evacuación atmosférica y condiciones quasi-estacionarias, para diferentes valores de $4 f L / D$ y relaciones del área del viento $A / A_{c}$

los resultados para $c_{0}(0) t_{f} / L=50$ están muy próximos a los correspondientes al modelo quasi-estacionario de Bar-Meir et al. (1997), también representados en la Figura 5.22.

Obsérvese que, para condiciones quasi-estacionarias, la masa de aire en el interior del cilindro en un $t / t_{f}$ dado disminuye con $A / \mathcal{A}$, aunque por encima de un valor crítico de la relación $A / \mathcal{A}$, más o menos definido y que no depende de $t / t_{f}$, permanece aproximadamente constante. Esto sucede para cualquier valor del parámetro de fricción, aunque la relación $A / \mathcal{A}$ crítica aumenta con $4 f L / D$. Obsérvese que, para $4 f L / D=0$, la relación $A / \mathcal{A}$ crítica se puede considerar igual a 2 . Cuando los efectos de fricción son mayores, la pendiente de la curva $m(t) / m(0)$ representada en función de $A / \mathcal{A}$ es menos pronunciada, y la relación $A / \mathcal{A}$ crítica queda menos definida, aunque para $4 f L / D=5$ y 50 podemos considerar que toma valores en torno a 4 y 10 , respectivamente. A pesar de la ligera dependencia de $m(t) / m(0)=f\left(A / A_{\mathcal{C}}\right)$ con $4 f L / D$, anteriormente mencionada, los tres valores de $A / \mathcal{A}$ crítica indicados corresponden, aproximadamente, a un único valor de $A / A_{c}=1,2$, que coincide con el valor dado por Bar-Meir et al. (1997) para $4 f L / D=5$. Este resultado se puede ver con más detalle en la Figura 5.23, en la que se representan los resultados del modelo de Bar-Meir et al. (1997) para la fracción de masa 


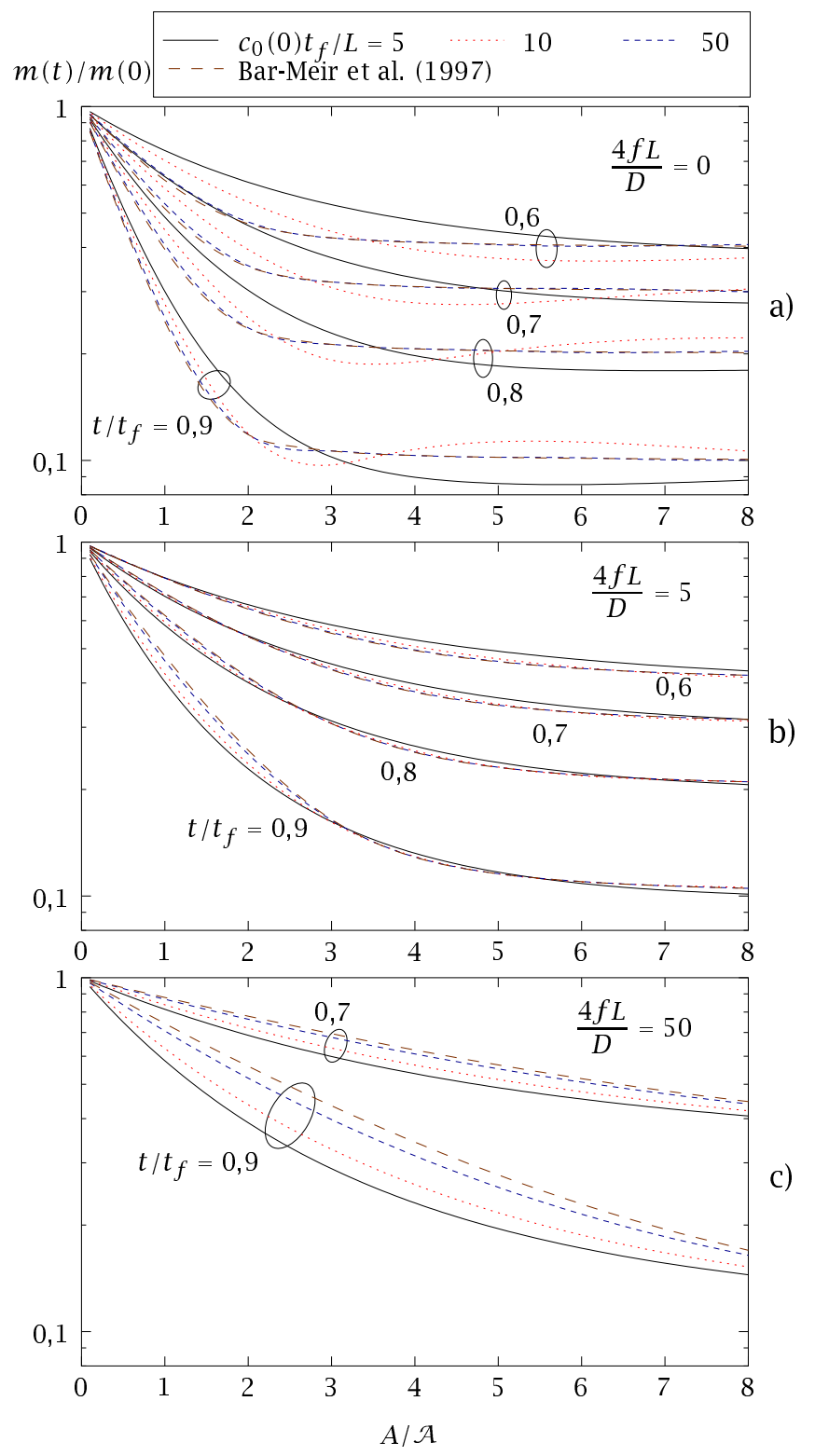

Figura 5.22: Fracción de masa de aire restante en el cilindro en función de $A / \mathcal{A}$, para evacuación atmosférica, en varios instantes, $t / t_{f}$, y diferentes valores de $c_{0}(0) t_{f} / L$. a) $4 f L / D=0$. b) $4 f L / D=5$. c) $4 f L / D=50$. 
de aire restante en el cilindro en $t / t_{f}=0,9$, en función de $A / A_{\mathcal{C}}$, para diferentes valores del parámetro $4 f L / D$.

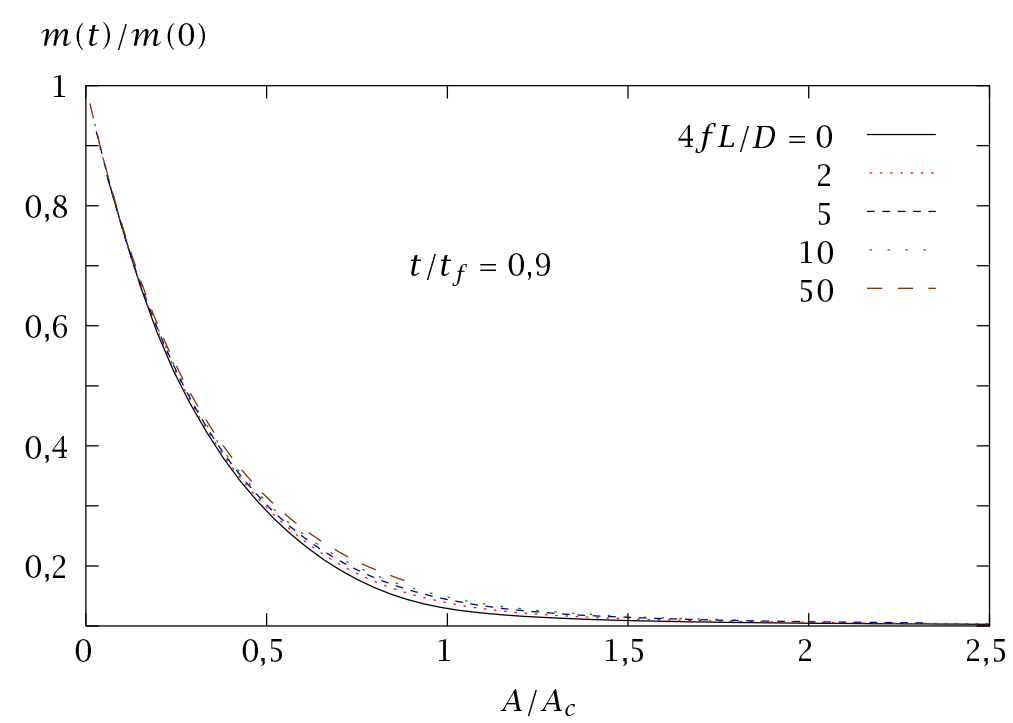

Figura 5.23: Fracción de masa de aire restante en el cilindro obtenida con el modelo quasi-estacionario de Bar-Meir et al. (1997) en $t / t_{f}=0,9$, en función de $A / A_{c}$, para evacuación atmosférica y diferentes valores del parámetro $4 f L / D$.

Para condiciones no estacionarias, la masa de aire en el cilindro puede no disminuir monótonamente con $A / \mathcal{A}$ para valores de este parámetro suficientemente grandes (Figura 5.22a), y la relación $A / \mathcal{A}$ óptima para la que la masa de aire en el cilindro es mínima, o por encima de la cual la masa de aire permanece aproximadamente constante, aumenta conforme disminuyen $c_{0}(0) t_{f} / L$ y $t / t_{f}$. En los casos representados en las Figuras $5.22 \mathrm{a}$ y $5.22 \mathrm{~b}$ (excepto para $4 f L / D=5, t / t_{f}=0,6$ y $c_{0}(0) t_{f} / L=5$, en el rango de $A / \mathcal{A}$ mostrado en la figura), se puede observar que es posible encontrar un rango de valores de $A / \mathcal{A}$ para los que la masa de aire restante en el cilindro es menor que la correspondiente al área crítica para el caso quasi-estacionario. La dependencia que la relación óptima del área tiene en $t / t_{f}$ introduce una incertidumbre adicional en la selección de un área apropiada del viento cuando el instante en el que el metal fundido alcanza la sección de entrada al viento no puede ser predicho a priori.

La Figura 5.24 se ha preparado para mostrar con más detalle la influencia de los efectos no estacionarios sobre la masa de aire restante en 


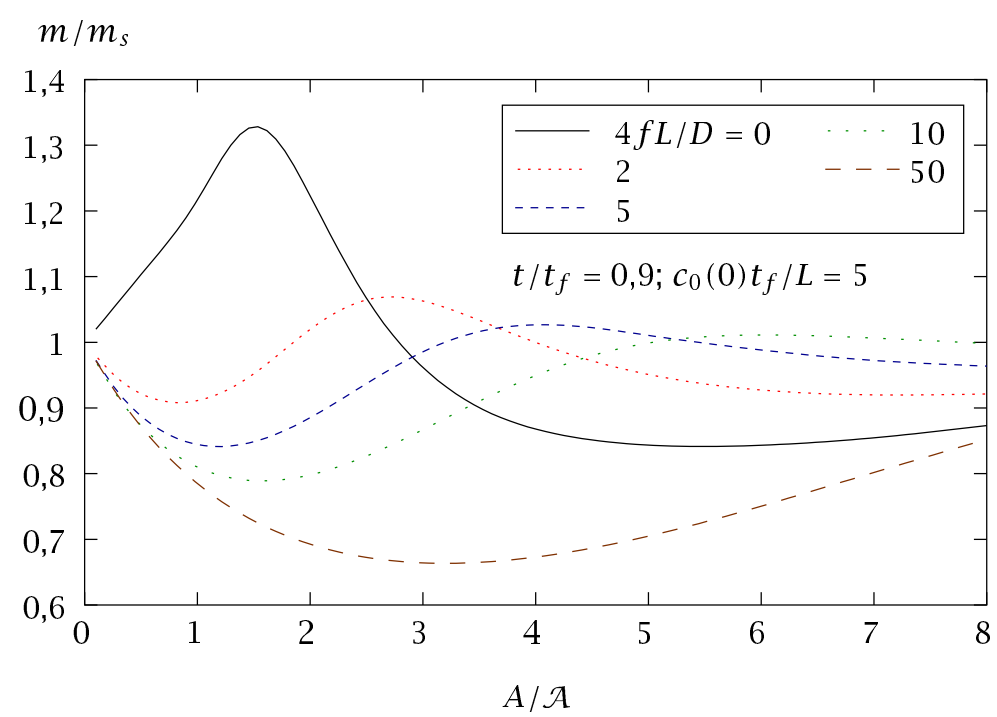

Figura 5.24: Relación entre la masa de aire en el cilindro en $t / t_{f}=0,9$ predicha por el modelo no estacionario para $c_{0}(0) t_{f} / L=5$ y la predicha por el modelo de Bar-Meir et al. (1997), en función de $A / \mathcal{A}$, para evacuación atmosférica y diferentes valores del parámetro de fricción.

el cilindro para evacuación atmosférica. En esta figura se representa la relación entre la masa de aire en el cilindro predicha por el modelo no estacionario y la predicha por el modelo quasi-estacionario en función de $A / \mathcal{A}$, para $t / t_{f}=0,9, c_{0}(0) t_{f} / L=5$ y diferentes valores del parámetro de fricción. El valor de $t / t_{f}=0,9$ se ha tomado como un valor representativo del instante en el que el metal fundido alcanza y obtura el viento. Aunque existen importantes efectos no estacionarios para valores de $A$ inferiores al valor crítico, como se puede ver en la Figura 5.22, los valores que se encuentran en un rango de áreas alrededor y por encima del valor crítico son los de mayor interés práctico. Para cada valor de $4 f L / D$, el valor crítico del área correspondiente a condiciones quasi-estacionarias se encuentra próximo al valor de $A / \mathcal{A}$ correspondiente al máximo local que se puede apreciar en las curvas de la Figura 5.24 (para $4 f L / D=50$ el máximo se sitúa fuera del rango de $A / \mathcal{A}$ representado en la figura). Se puede observar que los efectos no estacionarios tienden a hacer que $m$ sea mayor que $m_{s}$ para áreas ligeramente superiores al valor crítico y menor que $m_{s}$ para áreas incluso mayores. Las variaciones de $m$ con respecto a $m_{s}$ alrededor del área crítica son, aproximadamente, del orden del $25 \%, 7 \%$ y $3 \%$ para $4 f L / D=0,2$ y 5 , respectivamente. De lo ante- 
rior, se puede deducir que áreas del viento mayores que el valor crítico predicho por el modelo quasi-estacionario proporcionan importantes reducciones en la masa de aire residual en el cilindro, en especial, cuando los efectos de la fricción son menores.

\subsubsection{Evacuación al vacío}

Al igual que en la sección anterior, en las Figuras 5.25 y 5.26 se comparan, para condiciones de evacuación al vacío, los resultados de la presión en el cilindro en función del tiempo que se han obtenido con el modelo no estacionario y con el modelo quasi-estacionario de Bar-Meir et al. (1996). Los resultados de la Figura 5.25 se han obtenido despreciando los efectos de fricción, y los de la Figura 5.26 para un parámetro de fricción $4 f L / D=$ 5. El parámetro $c_{0}(0) t_{f} / L$ varía desde un valor igual a 5 en las Figuras 5.25 a y 5.26 a hasta 50 en las Figuras 5.25 b y $5.26 \mathrm{~b}$.

A partir de las Figuras 5.25 y 5.26, para condiciones de evacuación al vacío se pueden hacer consideraciones similares a las expuestas para las condiciones de evacuación atmosférica de la sección anterior. Como ya se ha mencionado, la presión en el cilindro y en cualquier punto del viento es inicialmente igual a $p_{0}(0)$. En $t=0$ (después de que el pistón haya cerrado el orificio de colada), la sección de salida del viento se conecta instantáneamente a un tanque de vacío, suficientemente grande, donde la presión se puede considerar constante en todo momento. A continuación el flujo alcanza el bloqueo sónico rápidamente. Para valores bajos de $A / \mathcal{A}$, la presión evoluciona en el cilindro de forma similar a como lo hace en el caso de evacuación atmosférica. Esto también ocurre para grandes valores de $A / \mathcal{A}$ durante un periodo de tiempo inicial, que es mayor para valores pequeños del parámetro $c_{0}(0) t_{f} / L$; después de este periodo, la presión aumenta más lentamente e inmediatamente y a continuación empieza a disminuir. También en este caso, como cabía esperar, los efectos no estacionarios llegan a ser menos importantes para grandes valores de $c_{0}(0) t_{f} / L$. Sin embargo, para valores de $c_{0}(0) t_{f} / L$ y $A / \mathcal{A}$ suficientemente grandes, la presión en el cilindro puede llegar a acercarse a la presión de vacío y oscilar alrededor de ella (Figura 5.25b). Para tales casos, los efectos de fricción tienden a hacer que la presión en el cilindro disminuya más lentamente, y como en el caso de evacuación atmosférica, tienden a amortiguar las anteriores oscilaciones (Figura 5.26b). El modelo quasi-estacionario de Bar-Meir et al. (1996) supone que el flujo está bloqueado sónicamente en todo instante, lo que sólo ocurre realmente para una presión de vacío muy pequeña y grandes valores de $A / \mathcal{A}$. En este trabajo se ha considerado una presión típica de vacío de $0,167 p_{0}(0)$ (25 pulgadas de mercurio, aproximadamente), por lo que el flujo puede no estar bloqueado, no sólo al principio del proceso de llenado, sino también para valores suficientemente grandes de $t / t_{f}$, en 

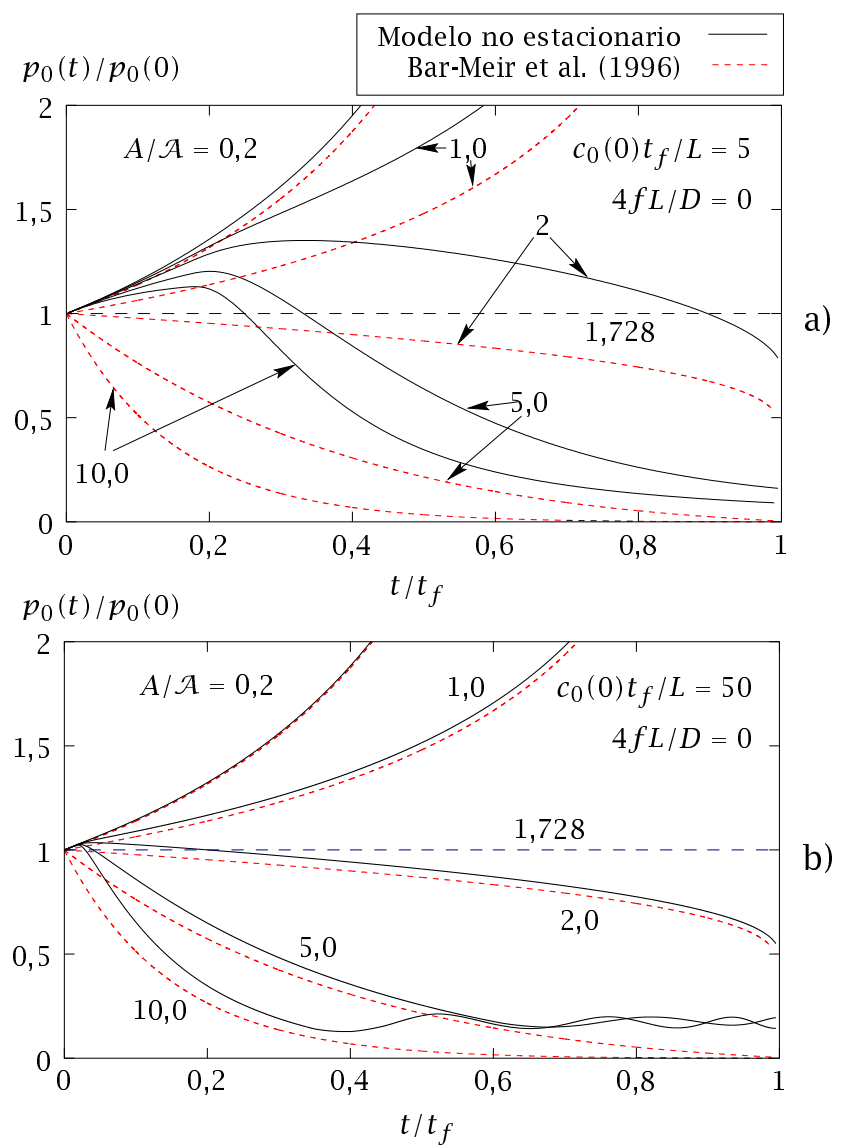

b)

Figura 5.25: Relación adimensional de la presión en el cilindro en función del tiempo adimensional para evacuación al vacío, $4 f L / D=0$ y diferentes valores de $A / \mathcal{A}$. a) $c_{0}(0) t_{f} / L=5$. b) $c_{0}(0) t_{f} / L=50$. 


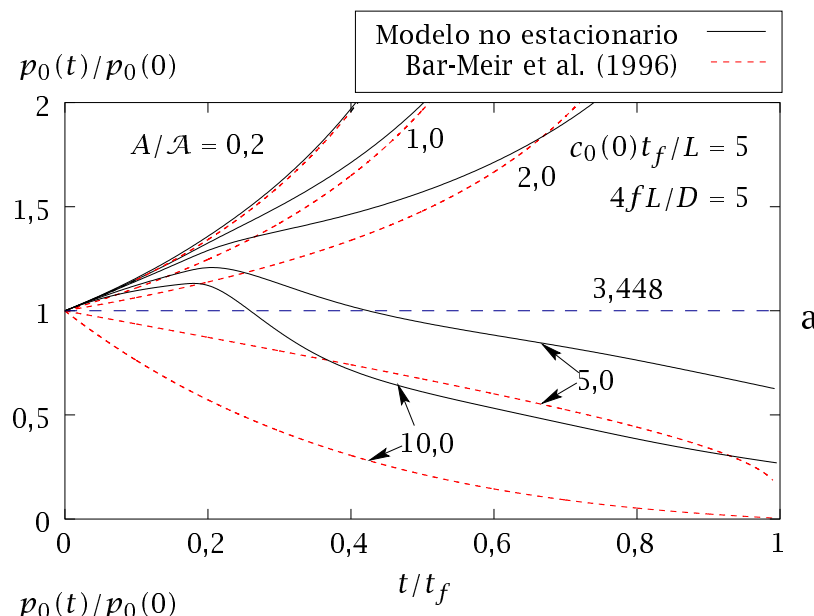

a)

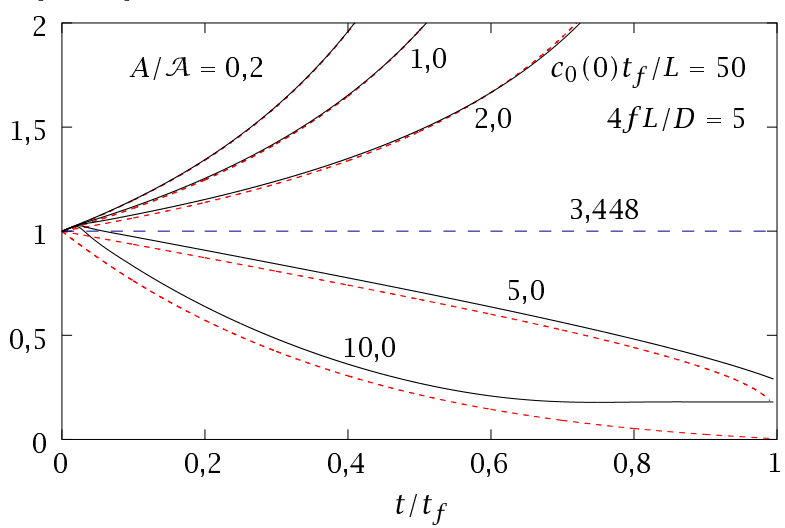

b)

Figura 5.26: Relación adimensional de la presión en el cilindro en función del tiempo adimensional para evacuación al vacío, $4 f L / D=5$ y diferentes valores de $A / \mathcal{A}$. a) $c_{0}(0) t_{f} / L=5$. b) $c_{0}(0) t_{f} / L=50$. 
particular para grandes valores de $c_{0}(0) t_{f} / L$ y $A / \mathcal{A}$, y valores pequeños de $4 f L / D$.

En los casos de las Figuras 5.25 y 5.26, un valor de la relación entre el área del viento y el área definida por la Ecuación (5.83), $A / A_{\mathcal{c}}$, igual a 1 corresponde a valores de $A / \mathcal{A}$ iguales a 1,728 y 3,448 , respectivamente, para los que las curvas correspondientes al modelo de Bar-Meir et al. (1996) se han dibujado con trazo de línea discontinuo.

Resultados similares a los de las Figuras 5.22 y 5.24 se representan en las Figuras 5.27 y 5.28 para condiciones de evacuación al vacío. Obsérvese que, por las razones expuestas anteriormente, los resultados para $c_{0}(0) t_{f} / L=500$ en la Figura 5.27, que se pueden considerar como quasi-estacionarios, sólo coinciden con los del modelo quasi-estacionario de Bar-Meir et al. (1996) para valores de $A / \mathcal{A}$ suficientemente pequeños. Obsérvese también que para un $t / t_{f}$ dado, como sucede para evacuación atmosférica, para condiciones quasi-estacionarias existe un valor crítico del área por encima del cual la masa de aire residual en el cilindro permanece aproximadamente constante. Este valor crítico, que además aumenta con $4 f L / D$ para una presión de vacío dada, como en el caso de evacuación atmosférica, también depende de $t / t_{f}$, disminuyendo conforme este parámetro aumenta. Para $t / t_{f}=0,9$ y $p_{\text {exit }} / p_{0}(0)=0,167$, por ejemplo, las relaciones críticas de área para $4 f L / D=0,5$, y 50 y condiciones quasi-estacionarias están en torno a 3,6 y 15, respectivamente, valores que corresponden a un único valor de $A / A_{c}$ alrededor de 1,8. Este resultado se puede ver con más claridad en la Figura 5.29, en la que se representa la fracción de la masa de aire restante en el cilindro en $t / t_{f}=0,9$, en función de $A / A_{c}$, para $c_{0}(0) t_{f} / L=500$ y diferentes valores del parámetro $4 f L / D$.

La masa de aire mínima que puede ser atrapada en el cilindro en un momento dado disminuye conforme la presión de vacío decrece, y se alcanza para áreas del viento iguales o mayores al valor de área crítico correspondiente a condiciones quasi-estacionarias, el cual aumenta conforme disminuye la presión de vacío. Esto último se puede ver en la Figura 5.30, en la que se representa la presión adimensional en el cilindro en función de $A / A_{c}$ para diferentes presiones de vacío y $t / t_{f}=0,9$. La línea continua corresponde a los resultados de Bar-Meir et al. (1996), válidos para valores de la presión de vacío suficientemente bajos", y las líneas discontinuas representan resultados numéricos para $c_{0}(0) t_{f} / L=500$, los cuales se pueden considerar como los correspondientes a las con-

\footnotetext{
" Para condiciones quasi-estacionarias y $4 f L / D=0$, de la Ecuación (5.2) se puede obtener que el viento dejará de estar bloqueado cuando la relación $\frac{p_{0}(t)}{p_{\text {exit }}}$ sea inferior a 1,893 . Este valor límite, a partir del cual el modelo de Bar-Meir et al. (1996) deja de ser válido, corresponde a valores de la presión adimensional $\frac{p_{0}(t)}{p_{0}(0)}$ iguales a 0,946, 0,568 y 0,316 para presiones adimensionales en el depósito de vacío $\frac{p_{\text {exit }}}{p_{0}(0)}$ de $0,5,0,3$ y 0,167, respectivamente (Figura 5.30).
} 

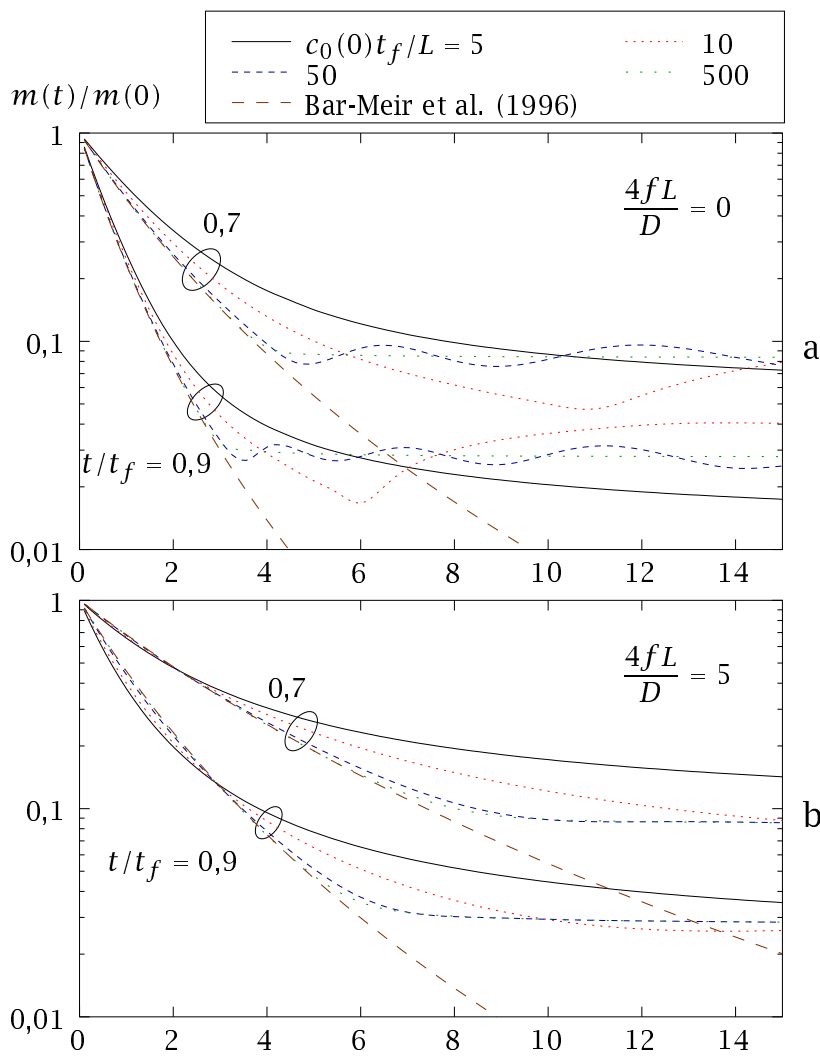

b)

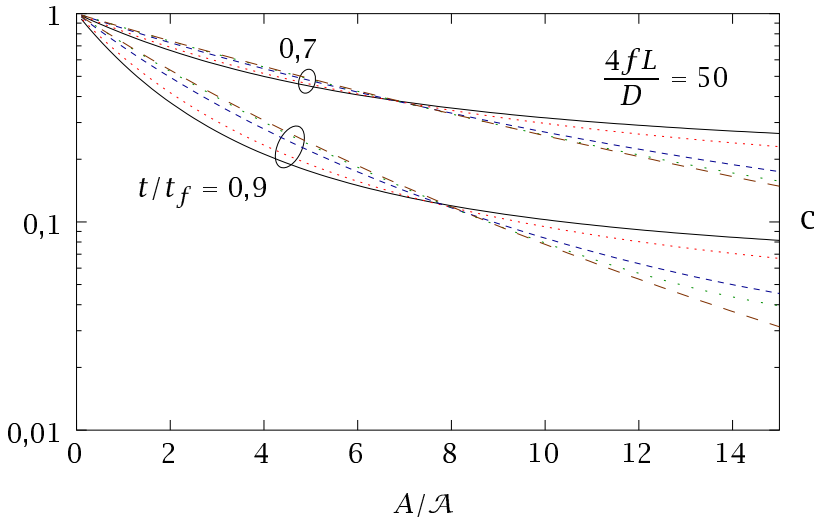

c)

Figura 5.27: Fracción de la masa de aire restante en el cilindro para varios instantes adimensionales, $t / t_{f}$, en función de $A / \mathcal{A}$, para evacuación al vacío y diferentes valores de $c_{0}(0) t_{f} / L$. a) $4 f L / D=0$. b) $4 f L / D=5$. c) $4 f L / D=50$. 


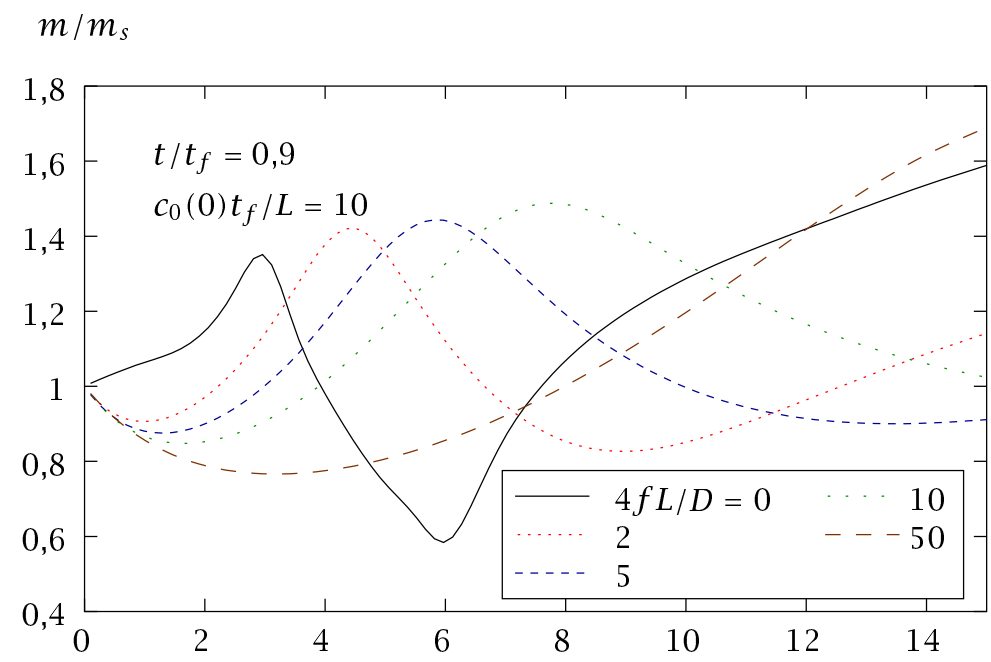

$A / \mathcal{A}$

Figura 5.28: Relación entre la masa de aire en el cilindro en $t / t_{f}=0,9$ predicha por el modelo no estacionario para $c_{0}(0) t_{f} / L=10$ y la obtenida considerando condiciones quasi-estacionarias $\left(c_{0}(0) t_{f} / L=500\right)$, en función de $A / \mathcal{A}$, para evacuación al vacío y diferentes valores del parámetro de fricción. 


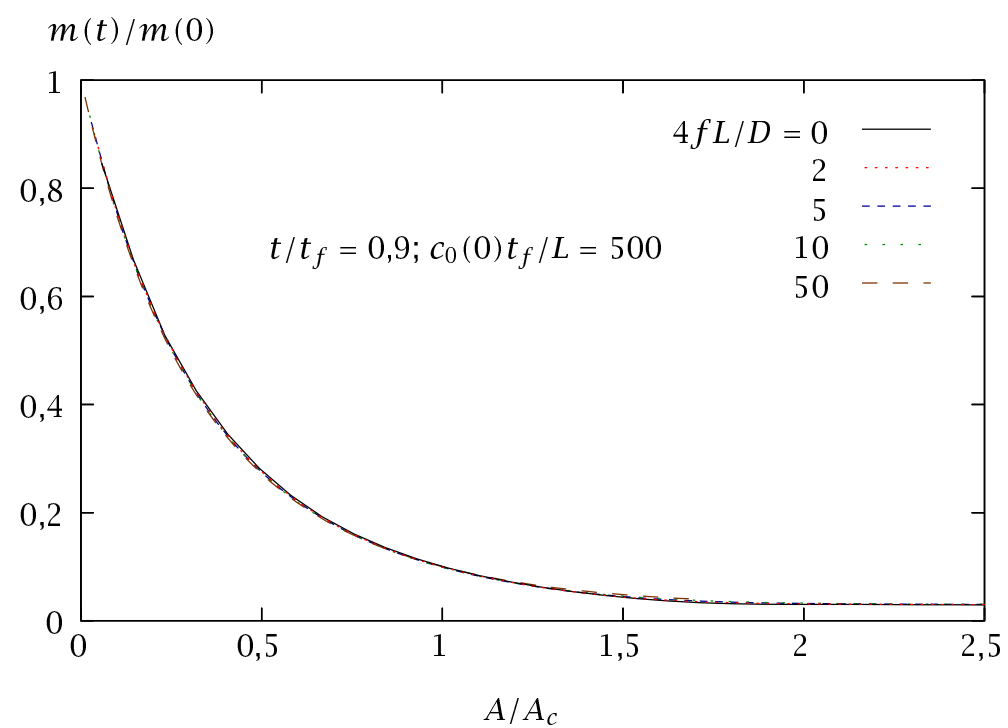

Figura 5.29: Fracción de la masa de aire restante en el cilindro en $t / t_{f}=0,9$, en función de $A / A_{c}$, para evacuación al vacío, $c_{0}(0) t_{f} / L=500$ y diferentes valores del parámetro $4 f L / D$. 


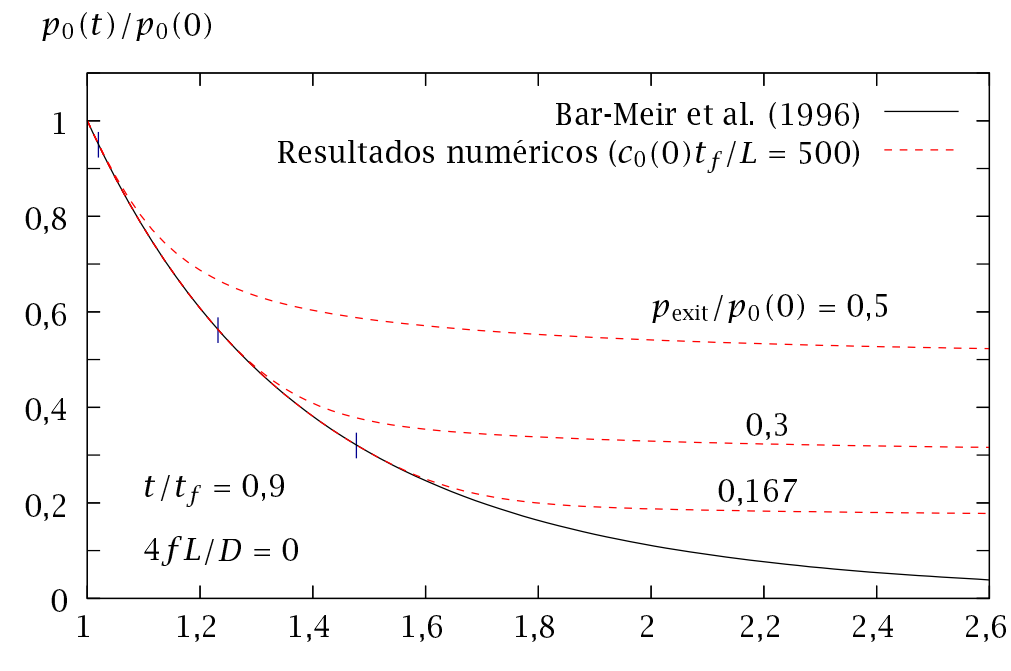

$A / A_{C}$

Figura 5.30: Presión adimensional en el cilindro en función de la relación $A / A_{c}$ predicha por el modelo numérico para $c_{0}(0) t_{f} / L=500$ y la correspondiente al modelo de BarMeir et al. (1996), para diferentes presiones de vacío y $t / t_{f}=0,9$. 
diciones quasi-estacionarias (los resultados para las condiciones quasiestacionarias también se pueden obtener mediante la integración numérica de la ecuación diferencial propuesta por Bar-Meir et al. (1997) (Ecuación (5.4)) para evacuación atmosférica, empleando las condiciones iniciales y de contorno apropiadas). Obsérvese que, en principio, es obviamente posible alcanzar valores de la presión en el cilindro, y por tanto valores de la masa de aire residual, tan bajos como se desee si la presión de vacío es suficientemente baja y se elige un valor de área del viento convenientemente grande para la presión de vacío seleccionada. En la práctica, las restricciones mencionadas en la introducción limitan el valor del área de la sección de los vientos.

Para condiciones no estacionarias, la comparación entre las Figuras 5.22 y 5.27 muestra que los resultados para evacuación al vacío son cualitativamente similares a los obtenidos para evacuación atmosférica, aunque los efectos no estacionarios en los primeros son mucho más importantes que en los segundos, particularmente en el caso de valores altos de $4 f L / D$. Esto se puede ver de forma más detallada en la Figura 5.28, correspondiente a un valor de $c_{0}(0) t_{f} / L=10$, para el que los efectos no estacionarios deberían, en principio, ser menos importantes que los de la Figura 5.24, correspondiente a $c_{0}(0) t_{f} / L=5$. Obsérvese que, para cada valor de $4 f L / D$, el máximo local de la relación $m / m_{s}$ se alcanza con una relación $A / \mathcal{A}$ próxima al valor crítico correspondiente al caso quasi-estacionario. En estos máximos, los efectos no estacionarios aumentan la masa de aire residual en el cilindro desde un 30\% (cuando no hay fricción) hasta casi un $50 \%$ para $4 f L / D=10$. Esto significa que, para reducir la masa de aire residual en el cilindro, puede ser necesario utilizar valores de área del viento sustancialmente superiores a los valores de área críticos determinados mediante los modelos quasi-estacionarios.

\subsection{Conclusiones}

Se ha analizado el flujo a través de los sistemas de evacuación de aire en los procesos de fundición por inyección a alta presión utilizando un modelo no estacionario para condiciones de evacuación atmosférica y al vacío. Como cabía esperar, los resultados numéricos del modelo muestran una muy buena concordancia con los resultados de modelos quasi-estacionarios previos para condiciones en las que los efectos no estacionarios son despreciables. Los resultados del modelo también se han comparado ampliamente con algunas soluciones analíticas de flujos unidimensionales compresibles no estacionarios con efectos de fricción y transferencia de calor, encontrándose una buena concordancia. Los resultados presentados en esta tesis muestran que, para amplios rangos de condiciones de operación, los efectos no estacionarios deben ser tenidos 
en cuenta para la selección de los parámetros de diseño que minimicen la cantidad de aire atrapado y de este modo reduzcan la porosidad en las piezas fabricadas. Para valores suficientemente bajos de $c_{0}(0) t_{f} / L$, dentro de los rangos encontrados en situaciones habituales de operación, se muestra que los efectos no estacionarios pueden afectar de forma importante a la masa de aire restante en la cavidad del molde en un instante dado. Como consecuencia, el valor mínimo del área del viento que proporciona una buena evacuación de aire, y por encima del cual la masa de aire residual en el cilindro no disminuye apreciablemente (aumentando, incluso, en algunos casos), puede ser considerablemente mayor que el valor crítico del área predicho por modelos quasi-estacionarios previos. Los efectos no estacionarios son, en general, mayores para condiciones de evacuación al vacío y, en el caso de áreas del viento alrededor del valor crítico correspondiente a las condiciones quasi-estacionarias, pueden incrementar la cantidad de aire residual en un 50\% o incluso más. Para evacuación atmosférica, los efectos no estacionarios para áreas del viento en torno al valor crítico correspondiente a las condiciones quasiestacionarias son menos importantes que los correspondientes a evacuación al vacío, particularmente, cuando los efectos de fricción en el viento son grandes, aunque, para condiciones no estacionarias suficientemente importantes y cuando los efectos de fricción son pequeños, pueden ser determinantes en la selección apropiada de los valores del área de la sección del viento. El análisis presentado y los resultados obtenidos hacen posible establecer criterios de diseño de los sistemas de evacuación que se utilizan en los procesos de fundición por inyección a alta presión. 



\section{A P Ít U L O 6}

\section{Conclusiones finales}

\subsection{Conclusiones}

En la presente tesis doctoral se ha realizado un estudio sistemático del comportamiento del metal fundido en la cámara de inyección y de la evacuación del aire contenido inicialmente en dicha cámara y en el molde, que tienen lugar en los procesos de fundición por inyección a presión. Este estudio se ha basado principalmente en dos modelos a partir de los cuales, siguiendo una metodología similar en ambos, se ha llevado a cabo un análisis de posibles condiciones de operación que puede utilizarse en la optimización de los procesos de inyección y evacuación de aire.

De forma general se puede concluir que los resultados de este estudio han permitido determinar valores de las variables de proceso y de diseño que minimizan la cantidad de aire atrapado y reducen en lo posible los tiempos de fabricación. Además se han obtenido valores límite de las condiciones de operación que deben evitarse para asegurar el correcto funcionamiento del proceso de inyección. Por lo tanto, con este estudio se ha contribuido al establecimiento de criterios que permitan la mejora de la calidad del producto y de la productividad del proceso. Por otra parte, los modelos propuestos constituyen herramientas útiles para el diseño del proceso de inyección, que pueden además ser aplicadas a procesos concretos, con máquinas de características y condiciones de operación determinadas, para evaluar su adecuación desde el punto de vista de la porosidad de las piezas fabricadas.

Para estudiar el flujo del metal fundido en el interior de la cámara de inyección se ha utilizado un modelo bidimensional en el que se despre- 
cian los efectos viscosos y se ha introducido la aproximación de aguas poco profundas, que ha sido resuelto numérica y analíticamente (según se consideren o no los efectos de reflexión de la ola de metal fundido en la cámara de inyección). Para evaluar la importancia de los efectos viscosos y no hidrostáticos, despreciados en el modelo anterior, el problema también se ha resuelto numéricamente mediante un código de elementos finitos con el que se determinó la posición de la superficie libre en cada instante mediante el método VOF, y en el que la malla de cálculo se movió y deformó usando una técnica similar al método Lagrangiano-Euleriano arbitrario. Por otro lado, la evacuación de aire durante el proceso de inyección se ha estudiado mediante un modelo no estacionario en el que el aire se ha considerado como un gas perfecto y el flujo de aire a través de los vientos se ha supuesto unidimensional. Este modelo se ha resuelto numéricamente usando un procedimiento similar al empleado para resolver el modelo de aguas poco profundas.

El modelo de aguas poco profundas se ha aplicado a dos tipos de leyes de movimiento del pistón que se podrían considerar como representativas de máquinas en las que la velocidad del pistón se programa en función de la posición del pistón (ley de aceleración exponencial) y del tiempo (ley de aceleración potencial). Se ha llevado a cabo en primer lugar un estudio analítico de los perfiles de ola de metal fundido, despreciando los efectos de reflexión, para amplios rangos de los parámetros relevantes del proceso, para los que se ha determinado el lugar y el instante en el que se forma una discontinuidad en la superficie libre del metal fundido. Ya que el tiempo de llenado debe ser lo más pequeño posible para mejorar la productividad y evitar solidificaciones prematuras del metal fundido durante el llenado del molde, se ha impuesto como condición límite el que la ola empiece a romperse justo en la pared final de la cámara de inyección, habiéndose comprobado que el uso de la ley de aceleración exponencial origina perfiles de ola más inclinados en el momento de formación de la discontinuidad que los que produce la ley de aceleración potencial, lo que da lugar a una reducción tanto del tiempo de llenado como del volumen de aire atrapado. También se ha observado que ninguna de estas leyes permite elegir un conjunto de parámetros que elimine totalmente el aire del interior de la cámara de inyección en el momento en el que la ola de metal fundido alcanza la pared final. Los resultados obtenidos analíticamente para las dos leyes de movimiento consideradas han permitido determinar, para unas condiciones de proceso y características geométricas de la cámara de inyección dadas, relaciones en las que intervienen los parámetros que definen la ley de movimiento del pistón que evitan el atrapamiento de aire por rotura de la ola reduciendo en lo posible el tiempo de inyección. Algunos de los resultados para el parámetro de aceleración $\alpha$ de la ley exponencial obtenidos de esta forma se han comparado, para distintos valores de la fracción inicial de 
llenado de la cámara de inyección, con los valores de este parámetro que minimizan el aire atrapado obtenidos experimentalmente por Duran et al. (1991) en una cámara de inyección de sección circular. Aunque habría sido necesario disponer de más resultados experimentales, y a pesar de las incertidumbres existentes en la comparación, se ha observado una buena concordancia entre ellos. Para estas mismas condiciones, las predicciones óptimas del parámetro de aceleración $\alpha$ obtenidas por Tszeng y Chu (1994), aunque ligeramente superiores a los valores obtenidos en este trabajo, muestran una concordancia cualitativa excelente y un error medio del orden del $25 \%$.

La aplicación del modelo de aguas poco profundas teniendo en cuenta los efectos de reflexión de la ola de metal fundido contra la pared final de la cámara de inyección ha permitido determinar, para un amplio rango de los parámetros que caracterizan la ley de aceleración exponencial, el área ocupada por el aire en la cámara de inyección en el momento en el que el metal fundido alcanza la entrada hacia la cavidad del molde. Los resultados obtenidos han facilitado la determinación de parámetros de aceleración que satisfagan el doble compromiso de reducción del aire atrapado y de los tiempos de inyección. La aceptable concordancia entre los parámetros así obtenidos y los correspondientes al modelo que no tiene en cuenta la reflexión de la ola confirma la validez de éste para conseguir predicciones cualitativamente correctas de los parámetros óptimos del proceso.

Los resultados obtenidos del estudio analítico realizado sobre las leyes de aceleración exponencial y potencial plantearon la posibilidad de determinar una nueva ley que satisfaga el doble objetivo de reducir el aire atrapado de la cámara de inyección y minimizar el tiempo de llenado. Consecuentemente, se ha determinado una nueva ley de movimiento que permitiría obtener, según el modelo analítico, un perfil de ola completamente vertical en el instante en el que ésta alcanza la pared final de la cámara de inyección para cualquier valor de la fracción inicial de llenado. Esto evitaría cualquier reflexión parcial de la ola, reduciendo a cero la cantidad de aire atrapado en el momento del impacto, y minimizaría el tiempo de llenado.

El modelo analítico descrito en el Capítulo 2 ha permitido también determinar las condiciones límite de operación que no deben alcanzarse para evitar que la ola pueda romperse antes de que el metal fundido alcance el techo de la cámara de inyección (condición que impone un límite mínimo de la fracción inicial de llenado) o que el metal fundido pueda ser expulsado a través del orificio de colada (condición que determina un límite máximo de la fracción inicial de llenado). Se ha encontrado que las leyes de aceleración potencial y exponencial requieren fracciones iniciales de llenado que en ningún caso deben ser inferiores a 0,195, mientras que con la nueva ley, y para cualquier valor de la fracción inicial de lle- 
nado, es posible alcanzar la velocidad crítica del pistón antes de que se inicie la rotura de la ola. Por otro lado, se ha comprobado que esta ley ofrece mayores restricciones con respecto al máximo valor de la fracción inicial de llenado que se podría utilizar para evitar que el metal fundido sea expulsado a través del orificio de colada. A pesar de esta limitación, la esperada reducción del volumen de aire atrapado y del tiempo de inyección, y la posibilidad de usar fracciones iniciales de llenado menores, hacen que la nueva ley propuesta en este trabajo presente importantes ventajas sobre otras leyes de aceleración del pistón utilizadas habitualmente en procesos de fundición a presión.

Se ha observado que los efectos no hidrostáticos, despreciados en los modelos de aguas poco profundas, pueden llegar a ser importantes una vez que el metal fundido alcanza el techo o la pared final de la cámara de inyección. En general, los resultados numéricos que se han obtenido considerando estos efectos predicen perfiles de ola más suaves y un ligero retardo de la rotura de la ola de metal fundido, en particular para fracciones iniciales de llenado altas. Estas discrepancias provocan que el valor mínimo del aire atrapado obtenido teniendo en cuenta los efectos no hidrostáticos se consiga normalmente con aceleraciones ligeramente superiores a las predichas por el modelo de aguas poco profundas. Para condiciones en las que estos efectos pueden considerarse despreciables, la concordancia entre las predicciones del modelo numérico de elementos finitos y las correspondientes al modelo de aguas poco profundas es muy buena, como era previsible. A pesar de las diferencias encontradas en la forma de la superficie libre cerca de la pared final de la cámara de inyección, las predicciones del volumen de aire atrapado obtenidas con el modelo de aguas poco profundas describen razonablemente bien la influencia de los parámetros de aceleración y de la fracción inicial de llenado sobre dicho volumen, por lo que este modelo puede constituir una herramienta sencilla y útil para elegir condiciones de operación que reduzcan el aire atrapado y que, a la vez, mantengan el tiempo de llenado suficientemente bajo.

La modelización numérica del flujo en la cámara de inyección ha permitido comprobar que, bajo ciertas condiciones de operación, puede formarse un chorro junto a la pared superior de la cámara como resultado de la interacción que tiene lugar entre ésta y la parte superior del frente de ola de metal fundido. Se ha comprobado que los efectos viscosos deberían ser tenidos en cuenta para modelar apropiadamente este chorro. Se ha encontrado que la nueva ley de aceleración propuesta acentúa la tendencia, con respecto a las otras leyes estudiadas, a la formación de chorros en el techo de la cámara de inyección, en particular para valores altos de la fracción inicial de llenado y de la relación entre la longitud y la altura de la cámara de inyección, lo que en principio contribuye a aumentar la ingestión de aire. A pesar de este inconveniente, los resultados 
para el volumen de aire atrapado para diferentes fracciones iniciales de llenado, estimados utilizando el modelo de elementos finitos, teniendo en cuenta por tanto los efectos viscosos y no hidrostáticos, se pueden considerar aceptablemente próximos a la predicción analítica de aire atrapado nulo que proporciona el modelo de aguas poco profundas para la nueva ley de aceleración propuesta.

En lo que se refiere al estudio de la evacuación de aire a través de los vientos, el modelo no estacionario utilizado para analizar condiciones de evacuación atmosférica y al vacío ha permitido establecer criterios para diseñar correctamente los sistemas de evacuación de aire que se usan en los procesos de fundición por inyección a presión. Para situaciones en las que los efectos no estacionarios son despreciables, se han comparado los resultados de este modelo con los resultados de modelos quasiestacionarios previos, habiéndose obtenido una muy buena concordancia entre ellos. También se han determinado rangos de los parámetros relevantes de proceso para los que los efectos no estacionarios pueden ser importantes.

Para condiciones quasi-estacionarias, se han obtenido los valores críticos del área del viento por encima de los cuales no se consigue una reducción adicional de la masa de aire residual en la cavidad del molde. Para condiciones de evacuación atmosférica, se ha encontrado una excelente concordancia entre el valor crítico del área de la sección transversal del viento obtenido con este modelo, que aumenta con el coeficiente de fricción en el viento, y los valores predichos por Bar-Meir et al. (1997). Para condiciones de evacuación al vacío, se ha comprobado que los resultados de Bar-Meir et al. (1996) sólo son válidos para valores de la presión de vacío suficientemente bajos, y que, al igual que para el caso de evacuación atmosférica, existe un valor crítico del área del viento por encima del cual no se consigue una reducción adicional de la masa de aire residual. Este valor crítico, además de aumentar con el coeficiente de fricción para una presión de vacío dada, también depende de la relación entre el tiempo en el que se obtura la entrada del viento y el tiempo de llenado, disminuyendo conforme este parámetro aumenta. Esto introduce una incertidumbre adicional para diseñar correctamente los sistemas de evacuación de aire cuando se desconoce el instante en el que los vientos son obturados por el metal fundido.

Los resultados presentados en esta tesis han demostrado que existen rangos de condiciones habituales de operación en las que los efectos no estacionarios deben ser tenidos en cuenta en la elección de los parámetros de diseño que minimicen la cantidad de aire atrapado y de este modo reduzcan la porosidad en las piezas fabricadas. Aunque los efectos no estacionarios pueden ser relevantes en condiciones de evacuación atmosférica, sobre todo cuando la fricción y el parámetro $c_{0}(0) t_{f} / L$ son suficientemente bajos, son más importantes para condiciones de eva- 
cuación al vacío, en las que, para valores de áreas del viento alrededor del valor crítico obtenido a partir del modelo quasi-estacionario, la masa de aire residual puede aumentar incluso hasta un $50 \%$ o más cuando se tienen en cuenta dichos efectos. Tanto en sistemas de evacuación atmosférica como al vacío, el valor mínimo del área del viento que proporciona una buena evacuación de aire, y por encima del cual no se consigue una reducción adicional apreciable de la masa de aire residual en la cavidad del molde, depende del coeficiente de fricción, aumentando conforme este coeficiente aumenta, y de $c_{0}(0) t_{f} / L$ y $t / t_{f}$, aumentando conforme estos parámetros disminuyen. Los valores del área crítica generalmente son mayores que los valores críticos obtenidos a partir del modelo quasi-estacionario, y la diferencia entre ambos aumenta al aumentar la no estacionariedad del proceso, consiguiéndose una reducción adicional de la masa de aire atrapado, por encima de la estimada a partir del modelo quasi-estacionario.

\subsection{Desarrollos futuros}

Los resultados obtenidos en esta tesis sugieren la consideración de posibles líneas de investigación futuras. Aunque el estudio bidimensional del flujo de metal fundido en el plano de simetría de la cámara de inyección realizado en esta tesis describe razonablemente bien las características esenciales del flujo, sería interesante llevar a cabo un estudio tridimensional. Otra aproximación utilizada en esta tesis consiste en suponer que el metal fundido está en reposo en el instante en el que el pistón inicia su movimiento en la cámara de inyección. Esto es estrictamente cierto si el tiempo transcurrido desde que se vierte el metal en la cámara de inyección hasta que el pistón inicia el movimiento, conocido como tiempo de retardo de disparo, es lo suficientemente grande como para que el metal fundido alcance las condiciones de reposo. Si el pistón inicia la inyección demasiado pronto, las características del flujo en dicho instante, que dependerán entre otros factores del caudal de vertido del metal fundido a través del orificio de colada, del tamaño de éste y de la fracción inicial de llenado de la cámara de inyección, podrían tener una cierta influencia en la cantidad de aire atrapado. Por otro lado, aunque el trabajo desarrollado se centra principalmente en condiciones próximas a las óptimas, los efectos de la turbulencia y de tensión superficial que se han despreciado en esta tesis también podrían ser importantes en condiciones en las que se pueda producir una rotura anticipada de la ola o que originen la formación de chorros en el techo de la cámara de inyección. También se ha considerado que los fenómenos de transferencia de calor y solidificación no afectan apreciablemente al proceso de inyección, aunque pueden existir condiciones de operación o tipos de aleaciones para las 
que la altura del metal en solidificación en contacto con las paredes internas de la cámara y el cambio de las propiedades del metal (en especial la viscosidad) en dicha zona pueden ser importantes.

Las comparaciones de los resultados obtenidos en esta tesis con los experimentos realizados por otros autores para una ley de aceleración exponencial en una cámara de sección circular han mostrado una buena concordancia entre ellos. Sin embargo, sería interesante disponer de más resultados experimentales obtenidos, tanto en máquinas FIAP como en condiciones de semejanza con agua, para estudiar otras leyes de aceleración y determinar la influencia sobre la cantidad de aire atrapado de variables despreciadas en esta tesis tales como la temperatura de colada, el tiempo de retardo de disparo, el precalentamiento de los moldes, el tipo de aleación o el tipo de lubricantes empleados en los moldes.

Con respecto a la evacuación de aire, se ha determinado, para condiciones de evacuación atmosférica y al vacío, el valor apropiado del área de la sección transversal de los vientos considerando un valor del coeficiente de fricción constante. El modelo numérico de evacuación de aire desarrollado en esta tesis podría ser extendido con facilidad para considerar un coeficiente de fricción en el viento variable, que dependerá entre otros factores de la geometría del viento y de las propiedades del flujo de aire en cada instante. También sería interesante estudiar con este mismo modelo el flujo de aire a través del viento para distintas geometrías de la sección longitudinal del viento. Por otro lado, puede ser también interesante estudiar la reacción del oxígeno del aire inicial de la cámara de inyección y del molde con el metal fundido, ya que podría reducir el volumen de aire que debe ser evacuado, si bien esta reducción de volumen se puede ver compensada por la evaporación de lubricantes o por la precipitación de otros gases como el hidrógeno durante el llenado o la solidificación del metal fundido.

Desafortunadamente, no se han encontrado en la literatura resultados experimentales que permitan contrastar cuantitativamente los resultados del modelo propuesto y de otros modelos de evacuación de aire desarrollados hasta ahora, por lo que sería necesaria una investigación experimental detallada de la evacuación de aire en los procesos de fundición por inyección a presión. 



\section{Apéndice A. Ecuaciones características y condiciones de compatibilidad}

El método de las características se puede considerar como el procedimiento natural para resolver sistemas de ecuaciones hiperbólicas con dos variables independientes, ya que mediante una elección apropiada de las coordenadas del sistema, la diferenciación de las ecuaciones del problema es más sencilla. A este nuevo sistema se le llama sistema de coordenadas características y tiene la propiedad de que cada ecuación es diferenciable en una única dirección, coincidiendo esta dirección con la de las coordenadas características.

\section{A.1 Flujo en aguas poco profundas}

Las ecuaciones que describen el problema bidimensional tratado en los Capítulos 2 y 3 se pueden expresar como (Acheson, 1990)

$$
\begin{gathered}
\frac{\partial u}{\partial t}+u \frac{\partial u}{\partial x}+v \frac{\partial u}{\partial y}=-\frac{1}{\rho} \frac{\partial p}{\partial x}, \\
\frac{\partial v}{\partial t}+u \frac{\partial v}{\partial x}+v \frac{\partial v}{\partial y}=-\frac{1}{\rho} \frac{\partial p}{\partial y}-g,
\end{gathered}
$$




$$
\frac{\partial u}{\partial x}+\frac{\partial v}{\partial y}=0
$$

En estos capítulos se han resuelto las Ecuaciones (A.1), (A.2) y (A.3) utilizando la aproximación de aguas poco profundas en la que se considera que la altura típica del metal fundido, $h_{0}$, es mucho más pequeña que la longitud típica de la ola $L$ (Figura 2.2)

$$
h_{0}<<L \text {. }
$$

Con esta aproximación, la componente vertical de la aceleración $\mathrm{D} v / \mathrm{D} t$ de la Ecuación (A.2) se puede despreciar frente a $g$. Considerando esta simplificación, la Ecuación (A.2) resulta

$$
\frac{\partial p}{\partial y}=-\rho g
$$

Integrando y aplicando la condición $p=p_{0}$ en la superficie libre del metal fundido $y=h(x, t)$ se puede obtener la distribución de la presión como

$$
p=p_{0}+\rho g(h-y) .
$$

De esta forma la Ecuación (A.1) se puede expresar como

$$
\frac{\mathrm{D} u}{\mathrm{D} t}=-g \frac{\partial h}{\partial x},
$$

de donde se deduce que si la componente horizontal de la velocidad, $u$, es inicialmente independiente de $y$, entonces $u$ es independiente de $y$ en cualquier instante. Por lo tanto la Ecuación (A.1) se puede simplificar y expresar del siguiente modo

$$
\frac{\partial u}{\partial t}+u \frac{\partial u}{\partial x}=-g \frac{\partial h}{\partial x}
$$

donde $u$ y $h$ son funciones de $x$ y $t$ solamente.

Integrando ahora la Ecuación (A.3) con respecto a $y$ e imponiendo la condición $v=0$ en $y=0$ se obtiene

$$
v=-\frac{\partial u}{\partial x} y .
$$

Si definimos la cantidad $F(x, y, t)$ como la diferencia $y-h(x, t)$, para todas las partículas que se encuentren en la superficie libre el parámetro $F(x, y, t)$ permanecerá constante e igual a cero. Como $\mathrm{D} F / \mathrm{D} t=0$ en la superficie libre, podemos escribir que

$$
\frac{\partial F}{\partial t}+(\mathbf{u} \cdot \nabla) F=-\frac{\partial h}{\partial t}-u \frac{\partial h}{\partial x}+v=0
$$


para $y=h(x, t)$, e introduciendo esta expresión en la Ecuación (A.9) se obtiene

$$
\frac{\partial h}{\partial t}+u \frac{\partial h}{\partial x}+h \frac{\partial u}{\partial x}=0
$$

Introduciendo $c(x, t)=(g h)^{1 / 2}$ en las Ecuaciones (A.8) y (A.11) se puede escribir, respectivamente,

$$
\begin{gathered}
\frac{\partial u}{\partial t}+u \frac{\partial u}{\partial x}+c \frac{\partial(2 c)}{\partial x}=0 \\
\frac{\partial(2 c)}{\partial t}+u \frac{\partial(2 c)}{\partial x}+c \frac{\partial u}{\partial x}=0
\end{gathered}
$$

y sumando y restando estas ecuaciones se obtienen, respectivamente,

$$
\begin{aligned}
& {\left[\frac{\partial}{\partial t}+(u+c) \frac{\partial}{\partial x}\right](u+2 c)=0,} \\
& {\left[\frac{\partial}{\partial t}+(u-c) \frac{\partial}{\partial x}\right](u-2 c)=0,}
\end{aligned}
$$

que son las ecuaciones resueltas analítica y numéricamente en los Capítulos 2 у 3.

Para obtener el nuevo sistema de ecuaciones en coordenadas características correspondiente al problema descrito por las Ecuaciones (A.12) y (A.13) obtenidas mediante la aproximación de aguas poco profundas, se van a sumar estas ecuaciones multiplicadas, respectivamente, por los parámetros desconocidos $\sigma_{1}$ y $\sigma_{2}$

$$
\sigma_{1}\left[\frac{\partial u}{\partial t}+u \frac{\partial u}{\partial x}+c \frac{\partial(2 c)}{\partial x}\right]+\sigma_{2}\left[\frac{\partial(2 c)}{\partial t}+u \frac{\partial(2 c)}{\partial x}+c \frac{\partial u}{\partial x}\right]=0,
$$

y reagrupando términos se puede obtener

$$
\left(\frac{\partial u}{\partial x}+\frac{\sigma_{1}}{u \sigma_{1}+c \sigma_{2}} \frac{\partial u}{\partial t}\right)+\frac{2 c \sigma_{1}+2 u \sigma_{2}}{u \sigma_{1}+c \sigma_{2}}\left(\frac{\partial c}{\partial x}+\frac{2 \sigma_{2}}{2 c \sigma_{1}+2 u \sigma_{2}} \frac{\partial c}{\partial t}\right)=0
$$

Si las dos variables dependientes $u(x, t)$ y $c(x, t)$ son funciones continuas, sus diferenciales totales se pueden expresar del siguiente modo

$$
\begin{aligned}
& \mathrm{d} u=\frac{\partial u}{\partial x} \mathrm{~d} x+\frac{\partial u}{\partial t} \mathrm{~d} t \\
& \mathrm{~d} c=\frac{\partial c}{\partial x} \mathrm{~d} x+\frac{\partial c}{\partial t} \mathrm{~d} t
\end{aligned}
$$

y reagrupando términos se obtiene

$$
\frac{\mathrm{d} u}{\mathrm{~d} x}=\frac{\partial u}{\partial x}+\frac{\mathrm{d} t}{\mathrm{~d} x} \frac{\partial u}{\partial t}
$$




$$
\frac{\mathrm{d} c}{\mathrm{~d} x}=\frac{\partial c}{\partial x}+\frac{\mathrm{d} t}{\mathrm{~d} x} \frac{\partial c}{\partial t} .
$$

Las características de las Ecuaciones (A.12) y (A.13) se definen como las curvas en el plano $x t$ cuya pendiente se puede obtener de los coeficientes de los términos $\frac{\partial u}{\partial t}$ y $\frac{\partial c}{\partial t}$ que se encuentran en la Ecuación (A.17). Por lo tanto, las Ecuaciones (A.20) y (A.21) se pueden expresar

$$
\begin{gathered}
\frac{\mathrm{d} u}{\mathrm{~d} x}=\frac{\partial u}{\partial x}+\frac{\sigma_{1}}{u \sigma_{1}+c \sigma_{2}} \frac{\partial u}{\partial t}, \\
\frac{\mathrm{d} c}{\mathrm{~d} x}=\frac{\partial c}{\partial x}+\frac{2 \sigma_{2}}{2 c \sigma_{1}+2 u \sigma_{2}} \frac{\partial c}{\partial t},
\end{gathered}
$$

y sustituyendo en la Ecuación (A.17) se obtiene

$$
\left(u \sigma_{1}+c \sigma_{2}\right) \mathrm{d} u+\left(2 c \sigma_{1}+2 u \sigma_{2}\right) \mathrm{d} c=0,
$$

que es una ecuación diferencial total válida solamente a lo largo de las ecuaciones características definidas por las ecuaciones

$$
\begin{gathered}
\frac{\mathrm{d} t}{\mathrm{~d} x}=\lambda=\frac{\sigma_{1}}{u \sigma_{1}+c \sigma_{2}}, \\
\frac{\mathrm{d} t}{\mathrm{~d} x}=\lambda=\frac{2 \sigma_{2}}{2 c \sigma_{1}+2 u \sigma_{2}} .
\end{gathered}
$$

Si se resuelven las Ecuaciones (A.25) y (A.26) para los parámetros $\sigma_{1}$ y $\sigma_{2}$ se puede obtener el sistema de ecuaciones

$$
\left.\begin{array}{r}
\sigma_{1}(\lambda u-1)+\sigma_{2}(\lambda c)=0 \\
\sigma_{1}(2 \lambda c)+\sigma_{2}(2 \lambda u-2)=0
\end{array}\right\}
$$

en el que el determinante de los coeficientes de la matriz de estas ecuaciones debe ser cero para que la solución del sistema sea distinta a la solución trivial $\sigma_{1}=\sigma_{2}=0$ :

$$
\left|\begin{array}{cc}
(\lambda u-1) & \lambda c \\
2 \lambda c & 2(\lambda u-1)
\end{array}\right|=0
$$

de donde se obtiene la ecuación

$$
(\lambda u-1)^{2}-(\lambda c)^{2}=0,
$$

cuya solución proporciona las ecuaciones características

$$
\frac{\mathrm{d} x}{\mathrm{~d} t}=\frac{1}{\lambda}=u \pm c
$$


Sustituyendo ahora el parámetro $\sigma_{1}=-\sigma_{2}(\lambda u-1) /(\lambda c)$, obtenido de la Ecuación (A.27), en la Ecuación (A.24) y dividiendo por $\sigma_{2}$ se obtiene para $\lambda=1 /(u+c)$ :

$$
(\mathrm{d} u+2 \mathrm{~d} c)=0
$$

y $\operatorname{para} \lambda=1 /(u-c)$ :

$$
(\mathrm{d} u-2 \mathrm{~d} c)=0
$$

e integrando las Ecuaciones (A.31) y (A.32) se obtiene la ecuación de compatibilidad válida a lo largo de las características definidas por la Ecuación (A.30):

$$
u \pm 2 c=\text { constante. }
$$

\section{A.2 Flujo unidimensional no estacionario de un gas perfecto}

Siguiendo el mismo procedimiento descrito en la sección anterior, el nuevo sistema de ecuaciones en coordenadas características correspondiente al problema descrito por las Ecuaciones (5.11), (5.12) y (5.13) se obtendrá sumando las ecuaciones anteriores multiplicadas respectivamente por los parámetros desconocidos $\sigma_{1}, \sigma_{2}$ y $\sigma_{3}$

$$
\begin{aligned}
\sigma_{1}\left(\frac{\partial \rho}{\partial t}+u \frac{\partial \rho}{\partial x}\right. & \left.+\rho \frac{\partial u}{\partial x}+\rho u \frac{1}{A} \frac{\mathrm{d} A}{\mathrm{~d} x}\right)+\sigma_{2}\left(\rho \frac{\partial u}{\partial t}+\rho u \frac{\partial u}{\partial x}+\frac{\partial p}{\partial x}-\mathcal{F}\right) \\
& +\sigma_{3}\left[\frac{\partial p}{\partial t}+u \frac{\partial p}{\partial x}-c^{2}\left(\frac{\partial \rho}{\partial t}+u \frac{\partial \rho}{\partial x}\right)-(\gamma-1)(\rho q-u \mathcal{F})\right] .
\end{aligned}
$$

Reagrupando términos se puede obtener

$$
\begin{aligned}
\left(\rho \sigma_{1}+\rho u \sigma_{2}\right)\left(\frac{\partial u}{\partial x}+\right. & \left.\frac{\sigma_{2}}{\sigma_{1}+u \sigma_{2}} \frac{\partial u}{\partial t}\right)+\left(\sigma_{2}+u \sigma_{3}\right)\left(\frac{\partial p}{\partial x}+\frac{\sigma_{3}}{\sigma_{2}+u \sigma_{3}} \frac{\partial p}{\partial t}\right) \\
+ & \left(u \sigma_{1}-c^{2} u \sigma_{3}\right)\left(\frac{\partial \rho}{\partial x}+\frac{\sigma_{1}-c^{2} \sigma_{3}}{u \sigma_{1}-c^{2} u \sigma_{3}} \frac{\partial \rho}{\partial t}\right) \\
+ & {\left[\sigma_{1} \rho u \frac{1}{A} \frac{\mathrm{d} A}{\mathrm{~d} x}-\sigma_{2} \mathcal{F}-\sigma_{3}(\gamma-1)(\rho q-u \mathcal{F})\right]=0 }
\end{aligned}
$$

Al igual que en la sección anterior, las pendientes de las características se obtienen de los coeficientes de las derivadas $\frac{\partial u}{\partial t}, \frac{\partial p}{\partial t}$ y $\frac{\partial \rho}{\partial t}$ de la Ecuación (A.35):

$$
\begin{aligned}
& \frac{\mathrm{d} t}{\mathrm{~d} x}=\lambda=\frac{\sigma_{2}}{\sigma_{1}+u \sigma_{2}}, \\
& \frac{\mathrm{d} t}{\mathrm{~d} x}=\lambda=\frac{\sigma_{3}}{\sigma_{2}+u \sigma_{3}},
\end{aligned}
$$




$$
\frac{\mathrm{d} t}{\mathrm{~d} x}=\lambda=\frac{\sigma_{1}-c^{2} \sigma_{3}}{u \sigma_{1}-c^{2} u \sigma_{3}},
$$

Suponiendo que $u(x, t), p(x, t)$ y $\rho(x, t)$ son funciones continuas se puede escribir

$$
\begin{gathered}
\frac{\mathrm{d} u}{\mathrm{~d} x}=\frac{\partial u}{\partial x}+\frac{\sigma_{2}}{\sigma_{1}+u \sigma_{2}} \frac{\partial u}{\partial t}, \\
\frac{\mathrm{d} p}{\mathrm{~d} x}=\frac{\partial p}{\partial x}+\frac{\sigma_{3}}{\sigma_{2}+u \sigma_{3}} \frac{\partial p}{\partial t}, \\
\frac{\mathrm{d} \rho}{\mathrm{d} x}=\frac{\partial \rho}{\partial x}+\frac{\sigma_{1}-c^{2} \sigma_{3}}{u \sigma_{1}-c^{2} u \sigma_{3}} \frac{\partial \rho}{\partial t},
\end{gathered}
$$

y sustituyendo en la Ecuación (A.35) se obtiene

$$
\begin{aligned}
\rho\left(\sigma_{1}+u \sigma_{2}\right) \mathrm{d} u & +\left(\sigma_{2}+u \sigma_{3}\right) \mathrm{d} p+\left(u \sigma_{1}-c^{2} u \sigma_{3}\right) \mathrm{d} \rho \\
& +\left[\sigma_{1} \rho u \frac{1}{A} \frac{\mathrm{d} A}{\mathrm{~d} x}-\sigma_{2} \mathcal{F}-\sigma_{3}(\gamma-1)(\rho q-u \mathcal{F})\right] \mathrm{d} x=0,
\end{aligned}
$$

que es la ecuación de compatibilidad válida a lo largo de las características definidas por las Ecuaciones (A.36), (A.37) y (A.38).

Resolviendo las Ecuaciones (A.36), (A.37) y (A.38) para $\sigma_{1}, \sigma_{2}$ y $\sigma_{3}$ se puede obtener el sistema de ecuaciones

$$
\left.\begin{array}{r}
\sigma_{1}(\lambda)+\sigma_{2}(\lambda u-1)=0 \\
\sigma_{2}(\lambda)+\sigma_{3}(\lambda u-1)=0 \\
\sigma_{1}(\lambda u-1)+\sigma_{3} c^{2}(1-\lambda u)=0
\end{array}\right\}
$$

cuya solución para ser distinta a la trivial $\left(\sigma_{1}=\sigma_{2}=\sigma_{3}=0\right)$ debe satisfacer la condición

$$
\left|\begin{array}{ccc}
\lambda & (\lambda u-1) & 0 \\
0 & \lambda & (\lambda u-1) \\
(\lambda u-1) & 0 & -c^{2}(\lambda u-1)
\end{array}\right|=0
$$

de donde se obtiene la expresión

$$
(\lambda u-1)\left[(\lambda u-1)^{2}-c^{2} \lambda^{2}\right]=0,
$$

cuya solución proporciona las ecuaciones características

$$
\begin{gathered}
\frac{\mathrm{d} x}{\mathrm{~d} t}=\frac{1}{\lambda}=u, \\
\frac{\mathrm{d} x}{\mathrm{~d} t}=\frac{1}{\lambda}=u \pm c,
\end{gathered}
$$


y de la Ecuación (A.42), operando de igual forma que en la sección anterior para $\lambda=1 / u$, se obtiene

$$
\mathrm{d} p-c^{2} \mathrm{~d} \rho=(\gamma-1)\left(\frac{\rho q}{u}-\mathcal{F}\right) \mathrm{d} x
$$

y $\operatorname{para} \lambda=1(u \pm c)$

$$
\mathrm{d} p \pm \rho c \mathrm{~d} u=\left[-\frac{1}{A} \frac{\mathrm{d} A}{\mathrm{~d} x} \rho u c^{2} \pm c \mathcal{F}+(\gamma-1)(\rho q-u \mathcal{F})\right] \mathrm{d} t .
$$

Las Ecuaciones (A.48) y (A.49) son las ecuaciones de compatibilidad válidas a lo largo de las características definidas por las Ecuaciones (A.46) y (A.47), respectivamente.

En la referencia Zucrow y Hoffman (1977) se puede ver de forma más detallada la aplicación del método de las características para éste y otros casos de dinámica de gases. 



\section{Bibliografía}

Acheson, D.J., 1990, Elementary Fluid Dynamics, Clarendon Press, Oxford.

Allsop, D.F. y Kennedy, D., 1983, Pressure DieCasting part 2: The Technology of the Casting and the Die. Pergamon Press, Oxford.

Ampuero, J., Charbon, Ch., Hoadley, A.F.A. y Rappaz, M.A., 1991, "Modeling of Microporosity Evolution During the Solidification of Metallic Alloys," Materials Processing in the Computer Age, pp. 377-388.

Amsden, A.A. y Harlow, F.H., 1970, "The SMAC Method, A Numerical Technique for Calculating Incompressible Flows," Technique Report LA-4370. Los Alamos Scientific Laboratory.

Auto Strategies International, 1994, World Automotive Market Report, pp. 17.

Bar-Meir, G., 1995, “On Gas/Air Porosity in Pressure Die Casting,” PhD thesis, University of Minnesota.

Bar-Meir, G., 1995, "Analysis of Mass Transfer Process in the Pore Free Technique," ASME Journal of Engineering Materials and Technology, v 117, pp. 215-219.

Bar-Meir, G., Eckert, E.R.G. y Goldstein, R.J., 1996, "Pressure Die Casting: A Model of Vacuum Pumping," ASME Journal of Manufacturing Science and Engineering, v 118, pp. 259-265. 
Bar-Meir, G., Eckert, E.R.G. y Goldstein, R.J., 1997, “Air Venting in Pressure Die Casting,” ASME Journal of Fluids Engineering, v 119, pp. 473-476.

Barton, H.K., 1963, "How to Vent Die Casting Dies," Foundry, julio, pp. 52-59.

Bennett, C.H., 1990, "Venting-The Die Casting Machine Becomes an Air Compressor," Die Casting Engineer, pp. 22-26.

Brevick, J.R., Armentrout, D.J. y Chu, Y., 1994, "Minimization of Entrained Gas Porosity in Aluminum Horizontal Cold Chamber Die Casting," Transactions of NAMRI/SME, 22, pp. 41-46.

Bühler Brothers, 1974, "Method for Pressure Casting," US Patent n 3964537.

Cai, R., 1998, "Some Explicit Analytical Solutions of Unsteady Compressible Flow," ASME Journal of Fluids Engineering, v 120, pp. 760-764.

Campbell, J., 1967, Pore Nucleation in solidifying Metals, The Solidification of Metals, Iron and Steel Inst., London, pp. 33-36.

Campbell, J., 1969, "Feeding Mechanisms in Castings," AFS Cast. Res. Met. Jnl., 5, marzo, pp. 1-8.

Campbell, J., 1991, Castings, Butterworth-Heinemann, Oxford.

Celik, I. y Zhang, W., 1995, "Calculation of Numerical Uncertainty Using Richardson Extrapolation: Application to Some Simple Turbulent Flow Calculations," ASME Journal of Fluids Engineering, v 117, pp. 439-445.

Chase, K.W. y Greenwood, W.H., 1988, "Design Issues in Mechanical Tolerances Analysis," Manufacturing Review, v 1, n 1, pp. 50-59.

Chorin, A. J., 1969, "On the Convergence of Discrete Approximations of the Navier-Stokes Equations," Math. Comput., v 23, pp. 341-353.

Chvorinov, N., 1940, Giesserei, 27, pp. 177-186,201-208,222-225.

Courant, R., Friedrichs, K.O. y Lewy, H., 1928, "Uber diee Partiellen Differenzialglichungen der Mathematischen Physik," Mathematische Annalen, v 100, pp. 32-74.

Dong, Z., Hu, W. y Xue, D., 1994, "New Production Cost-Tolerance Models for Tolerance Synthesis," ASME Journal of Engineering for Industry, v 116, pp. 199-206.

Doric, J.L. y Verma, S.K., 1988, “Squeeze Casting,” ASM Handbook, v 15, pp. 323-327. 
Draper, A.B., 1967, "Effect of Vent and Gate Areas on the Porosity of Die Casting," American Foundrymen's Society Transactions, 75 pp. 727 734.

Duran, M., Karni, Y., Brevick, J., Chu, Y. y Altan, T., 1991, "Minimization of Air Entrapment in the Shot Sleeve of a Die Casting Machine to Reduce Porosity," Technical Report ERC/NSM-C-91-31, The Ohio State University.

Faura, F., 1997, "Modelización Técnico-Económica de un Sistema de Moldeo de Aluminio a Alta Presión para la Fabricación de Piezas de Automoción," Revista de Metalurgia, v 33, n 3, pp. 381-390.

Faura, F., López, J. y Zamora, R., 1997, “Technical and Cost Analysis of a High Pressure Die Casting Operation for the Fabrication of Aluminum Automotive Parts," The Julian Szekely Memorial Symposium on Materials Precessing, ed. H.Y. Sohn, J.W. Evans y D. Apelian. Warrendale, P.A.: TMS, p. 731.

Flemings, M.C., 1974, Solidification Processing, McGraw-Hill Book Co., New York.

Garber, L.W., 1982, "Theoretical Analysis and Experimental Observation of Air Entrapment During Cold Chamber Filling," Die Casting Engineer, mayo-junio, pp. 14-22.

Ghomashchi, M.R., 1995, "High-Pressure Die Casting: Effect of Fluid Flow on the Microstructure of LM24 Die-Casting Alloy," Journal of Materials Processing Technology, v 52, n 2-4. pp. 193-206.

Hansen, P.N. y Sahm, P.R., 1988, "How to Model and Simulate the Feeding Process in Casting to Predict Shrinkage and Porosity Formation," Modeling of Casting and Welding Processes IV, Ed. A.F. Giamei y G.J. Abbaschian. Warrendale, P.A.: TMS, pp. 33-42.

Hirt, C.W., Amsden, A.A. y Cook, J.L., 1974, "An Arbitrary LagrangianEulerian Computing Method for all Flow Speeds," J. Comput. Phys., v 14, pp. 227-253.

Hirt, C.W. y Nichols, B.D., 1981, "Volume of Fluid (VOF) Method for the Dynamics of Free Boundaries," J. Comput. Phys., v 39, pp. 201-225.

Hirt, C.W., 1991, "A Flow-3d Study of the Importance of Fluid Momentum Mold Filling," Numerical Simulation of Casting Solidification in Automotive Applications, ed. Kim y Kim. Warrendale, P.A.: TMS, pp. 173-188.

Hu, J., Ramlingam, S., Meyerson, G., Eckert, E.R.G. y Goldstein, R.J., 1992, "Experiment and Computer Modelling of the Filling Flows in Pressure Die Casting," ASME/CIE Design, San Francisco, CA, v 1, pp. 401-410. 
Hwang, W.S. y Stoehr, R.A., 1983, "Fluid Flow Modeling for ComputerAided Design of Castings," J. Met. v 35, octubre, pp. 22-30.

Hwang, W.S. y Stoehr, R.A., 1988, "Modeling of Fluid Flow," ASM Metals Handbook, novena edición, v 15, pp. 867-876.

Kalpakjian, S., 1995, Manufacturing Engineering and Technology, Addison Wesley, New York.

Karni, Y., 1991, "Selection of Process Variables for Die Casting," PhD thesis, The Ohio State University, Ohio, USA.

Karni, Y., 1991, "Venting Design in Die Casting: An Analytical Approach," NADCA Congress and Exposition, Detroit, MI, G-T91-OC2.

Kim, C. y Kim, C-W., 1991, Numerical Simulation of Casting Solidification in Automotive Applications, ed. TMS, Pennsylvania (USA).

Kimio, K. y Pehlke, R.D., 1985, "Mathematical Modeling of Porosity Formation in Solidification," Met. Trans. 16B, junio, pp. 359-366.

Koster W. y Goehring, K., 1941, Giesserei, 28, (26), 521.

Kuo J.H. y Hwang W.S., 1998, "Flow Pattern Simulation in Shot Sleeve During Injection of Diecasting," American Foundrymen's Society Transactions, v 63, pp. 497-503.

Lamb, H., 1945, Hydrodynamics, Dover, New York.

Landau, L. y Lifshitz, E., 1959, Fluid Mechanics, Pergamon Press, Oxford.

Lee, W.J. y Woo, T.C., 1989, "Optimum Selection of Discrete Tolerances," ASME Journal of Mechanisms, Transmissions, and Automation in Design, v 1, n 3, pp. 168-179.

Lindberg, B., Hansen, P.N. y Hansen, S.F., 1991, "High Speed Filming of Mold Filling Process of Al Alloys in High Pressure Die Casting," Modeling of Casting, Welding and Advanced Solidification Processes V, ed. M. Rappaz, M.R. Özgü and K.W. Mahin. Warrendale, PA: TMS, pp. 763-769.

Lindsey, D. y Wallace, J.F., 1972, "Effect of Vent Size and Design, Lubrication Practice, Metal Degassing, Die Texturing and Filling of Shot Sleeve on Die Casting Soundness," Transactions of 7th SDCE International Die Casting Congress and Exposition, Chicago, USA, paper 10372, 1-15.

Lismont, H., et al., 1990, "PreCoCast and Cobapress," Seminar Aluminium mould castings using innovative technology, Meschede. 
Luis, L. y Draper, A.B., 1967, "Effect of Overflow Wells, Gating and Injection Parameters on the Porosity of a Die Casting," American Foundrymen's Society Transactions, 74 pp. 245-256.

Nichols, B.D., Hirt, C.W. y Hotchkiss, R.S., 1980, "SOLA-VOF, A Solution Algorithm for Transient Fluid Flow With Multiple Free Boundaries," Technical Report LA-8355, Los Alamos Scientific Laboratory.

Niyama, E., Uchida, T., Morikawa, M. y Saito, S., 1982, “A Method of Shrinkage Prediction and Its Application to Steel Casting Practice," AFS Int. Cast Metals Inst. Jnl., 7, pp. 52-63.

Ohnaka, I., 1993, "Modeling of Fluid Flow and Solidification in Casting," Modeling of Casting, Welding an Advanced Solidification Processes VI, ed. T.S. Piwonka, V. Voller y L. Katgerman. Warrendale, PA: TMS, pp. 337-348.

Ostermann, F. et al., 1993, Aluminium Materials Technology for Automobile Construction, Mechanical Engineering Publication Limited, London, U.K.

Pellini, W.S., 1953, "Factors with Determine Riser Adequacy and Feeding Range," American Foundrymen's Society Transactions, 61, pp. 61-80.

Piwonka, T.S. y Flemings, M.C., 1966, "Pore Formation in Solidification," Trans. Met. Soc. of AIME, 236, agosto, pp. 1157-1165.

Poirier, D.R., Yeum, K. y Maples, A.L., 1987, “A Thermodynamic Prediction For Microporosity Formation in Aluminum-Rich Al-Cu Alloys," Met. Trans., 18, noviembre, pp. 1979-1987.

Press, W.H.; Teukolsky, S.A.; Vetterling, W.T. y Flannery, B.P., 1988, Numerical Recipes in $C$, Cambridge University Press, Cambridge.

Rearwin, E.W., 1960, “Gating of Aluminium Diecasting," Foundry, Noviembre, pp. 106.

Sachs, B., 1952, "An Analytical Study of the Die Casting Process," PhD thesis, Columbia University.

Sant, F. y Backer, G., 1995, “Application of WRAFTS Fluid Flow Modeling Software to the Bench Mark Test Casting," Modeling of Casting, Welding and Advanced Solidification Processes VII, ed. M. Cross y J. Campbell. Warrendale, PA: TMS, pp. 983-990.

Scardovelli, R. y Zaleski, S., 1999, "Direct Numerical Simulation of FreeSurface and Interfacial Flow," Annual Review of Fluid Mechanics, 31, pp. 567-603. 
Shapiro, A.H., 1954, The Dynamics and Thermodynamics of Compressible Fluid Flow, The Ronald Press, New York.

Street, A.C., 1977, The Diecasting Book, Portcullis Press, Surrey, England.

Sutherland, G.H. y Roth, B., 1975, "Mechanism Design: Accounting for Manufacturing Tolerances and Cost in Function Generation Problems," ASME Journal of Engineering for Industry, v 98, pp. 283-286.

Szekely, J. y Themelis, N.J., 1971, Rate Phenomena in Process Metallugy, John Wiley \& Sons, New York.

Szekely, J., 1979, Fluid Flow Phenomena in Metals Processing, Academic Press, New York.

Thome, M.C. y Brevick, J.R., 1993, "Modeling Fluid Flow in Horizontal Cold Chamber Die Casting Shot Sleeves," American Foundrymen's Society Transactions, 101, pp. 343-348.

Thome, M.C. y Brevick, J.R., 1995, "Optimal Slow Shot Velocity Profiles for Cold Chamber Die Casting," NADCA Congress and Exposition, Indianapolis, paper T95-024.

Tsai, W. y Yue, D., 1996, “Computation of Non-Linear Free-Surface Flows," Annual Review of Fluid Mechanics, 28, pp. 247-278.

Tszeng, T.C. y Chu, Y.L., 1994, "A Study of Wave Formation in Shot Sleeve of a Die Casting Machine," ASME Journal of Engineering for Industry, 116(2), pp. 175-182.

Veinik, A.I., 1962, Theory of Special Casting Method, ASME, New York.

Veinik, A.I., 1966, "Thermodynamic Factors in Metal Injection: Effect of Friction on Gas Content and Quality," Transactions of the $4^{\text {th }}$ SDCE International Die Casting Exponsition \& Congress, Cleveland, Ohio, 103.

Wang, C.M., 1990, "Computer Modeling of Fluid Flow and Heat Transfer in Metal Castings," PhD thesis, University of Pittsburgn.

Welch, J.E., Harlow, F.H., Shanon, P.J. y Dally, B.T., 1965, “The MAC Method -A Computing Technique for Solving Viscous, Incompressible, Transient Fluid Flow Problems Involving Free Surfaces," Technical Report LA-3425, Los Alamos Scientific Laboratory.

Winter, D., 1994, Ward's Automotive Yearbook, pp. 23-27.

Zucrow, M.J. y Hoffman, J.D., 1977, Gas Dynamics, Wiley, New York. 


\section{Biografía del Autor}

Joaquín López Rodríguez nace en Alcantarilla (Murcia) en agosto de 1970. Cursa los estudios de Ingeniería Técnica Industrial (especialidad Mecánica) en la Escuela Universitaria Politécnica de Cartagena de la Universidad de Murcia concluyendo en 1992 con la calificación final de Sobresaliente, y recibe el premio extraordinario fin de carrera en Ingeniería Técnica Industrial y el segundo premio nacional de terminación de estudios. Ese mismo año inicia los estudios de Ingeniería Industrial (especialidad Mecánica de Máquinas) en la Escuela Politécnica Superior de la Universidad de Murcia concluyendo en 1995 con la calificación final de Sobresaliente y recibiendo el premio extraordinario fin de carrera en Ingeniería Industrial. Durante los cursos 1990/91, 1992/93 y 1993/94 colabora con el Departamento de Ingeniería de Materiales y Fabricación como Becario de Colaboración del Ministerio de Educación y Ciencia.

En 1995 inicia su actividad docente e investigadora como Profesor Ayudante de Escuela Universitaria en el Departamento de Ingeniería de Materiales y Fabricación (Área de Ingeniería de los Procesos de Fabricación) de la Universidad de Murcia. En la actualidad es Profesor Asociado a Tiempo Completo del mismo Departamento en la Universidad Politécnica de Cartagena y participa en los siguientes proyectos de investigación relacionados con la presente tesis doctoral:

- "Estudio técnico-económico y optimización de procesos de fundición por inyección a alta presión mediante técnicas computacionales" (TAP97-0489), financiado por la Comisión Interministerial de Ciencia y Tecnología (CICYT), 
- "Estudio experimental de la fase de inyección en procesos de fundición a presión" (1FD97-2333), cofinanciado por la CICYT y la Comisión Europea.

Los trabajos que se derivan del primer proyecto de investigación y que están relacionados con la presente tesis son los siguientes:

- Faura, F., López, J. y Zamora, R., 1997, “Technical and Cost Analysis of a High Pressure Die Casting Operation for the Fabrication of Aluminium Automotive Parts," The Julian Szekely Memorial Symposium on Materials Processing, ed. H.Y. Sohn, J.W. Evans y D. Apelian. Warrendale, P.A.: TMS, p. 731.

- López, J., Hernández, J., Faura, F., y Trapaga, G., 1998, "Shot Sleeve Wave Dynamics in the Slow Phase of Die Casting Injection," enviado a ASME Journal of Fluids Engineering. Aceptado.

- Hernández, J., López, J., Gómez, P. y Faura, F., 1999, “Influence of Non-Hydrostatic and Viscous Effects on Shot Sleeve Wave Dynamics in Die Casting Injection," Proceedings of the 3rd ASME/JSME Joint Fluids Engineering Conference. Forum on Advances in Free Surface and Interface Fluid Dynamics, FED-Vol. 248, San Francisco, USA.

- Hernández, J., López, J. y Faura, F., 1999, “Influence of Unsteady Effects on Air Venting in Pressure Die Casting," enviado a ASME Journal of Fluids Engineering. En revisión.

- Faura, F., López, J. y Hernández, J., 1999, “On the Optimum Plunger Acceleration Law in the Slow Shot Phase of Pressure Die Casting Machines," enviado a International Journal of Machine Tools \& Manufacture. En revisión.

- López, J., Hernández, J., Faura, F. y Gómez, P., 2000, "Effects of Shot Sleeve Wave Reflection on Air Entrapment in Pressure Die Casting Processes," ASME Fluids Engineering Conference. Symposium on Fluids in Manufacturing Processes. Boston, USA. Aceptado.

- Hernández, J., Gómez, P., López, J. y Faura, F., 2000, "Numerical Simulation of Free Surface Flows in Die Casting Injection Processes," Eighth International PHOENICS User Conference. Luxembourg. Aceptado.

Entre otros trabajos de investigación publicados y que están relacionadas con el Área de Ingeniería de los Procesos de Fabricación se citan los siguientes: 
- Faura, F., García, A., López, J. y Sebastián, M.A., 1996, "Blanking of Stainless Steel: Criteria of Wear Limitation," $5^{\text {th }}$ International Conference on Technology of Plasticity. ERC/NSM Advanced Technology of Plasticity, ed. T. Altan, v 2, pp. 645-654.

- Faura, F., López, J., Sebastián, M.A. y Luís, C., 1996, "Blanking of Stainless Steel: Tool Life Equation Model," $5^{\text {th }}$ International Conference on Technology of Plasticity. ERC/NSM Advanced Technology of Plasticity, ed. T. Altan, v 2, pp. 655-664.

- Faura, F., López, J. y Sanes, J., 1997, "Criterion for Tool Wear Limitation on Blanking 18-8 Stainless Steel Strips," Revista de Metalurgia, v 33, n 5, pp. 304-310.

- Faura, F., López, J., Sanes, J. y García, A., 1998, “Tool Life Equation for Blanking 18-8 Stainless Steel Strips," Revista de Metalurgia, v 34, n 4 , pp. 328-334.

- Faura, F., López, J., García, A. y Zamora, R., 1998, "Una Nueva Función Objetivo para la Optimización del Juego de Corte en Punzonado," Información Tecnológica, v 9, n 6, pp. 289-294.

- Faura, F., García, A. y López, J., 1999, "Estudio Experimental del Desgaste de Herramientas en el Punzonado de Chapa de Acero Inoxidable 18-8," Información Tecnológica, v 10, n 1, pp. 223-228. 